\title{
Global, regional, and national disability-adjusted life years (DALYs) for 306 diseases and injuries and healthy life expectancy (HALE) for 188 countries, 1990-2013: quantifying the epidemiological transition
}

\author{
GBD 2013 DALYs and HALE Collaborators
}

\section{Summary}

\begin{abstract}
Background-The Global Burden of Disease Study 2013 (GBD 2013) aims to bring together all available epidemiological data using a coherent measurement framework, standardised estimation methods, and transparent data sources to enable comparisons of health loss over time and across causes, age-sex groups, and countries. The GBD can be used to generate summary measures such as disability-adjusted life-years (DALYs) and healthy life expectancy (HALE) that make possible comparative assessments of broad epidemiological patterns across countries and time. These summary measures can also be used to quantify the component of variation in epidemiology that is related to sociodemographic development.
\end{abstract}

\begin{abstract}
Methods-We used the published GBD 2013 data for age-specific mortality, years of life lost due to premature mortality (YLLs), and years lived with disability (YLDs) to calculate DALYs and HALE for 1990, 1995, 2000, 2005, 2010, and 2013 for 188 countries. We calculated HALE using the Sullivan method; 95\% uncertainty intervals (UIs) represent uncertainty in age-specific death rates and YLDs per person for each country, age, sex, and year. We estimated DALYs for 306 causes for each country as the sum of YLLs and YLDs; 95\% UIs represent uncertainty in YLL and YLD rates. We quantified patterns of the epidemiological transition with a composite indicator of sociodemographic status, which we constructed from income per person, average years of schooling after age 15 years, and the total fertility rate and mean age of the population. We applied hierarchical regression to DALY rates by cause across countries to decompose variance related to the sociodemographic status variable, country, and time.
\end{abstract}

Findings-Worldwide, from 1990 to 2013, life expectancy at birth rose by $6 \cdot 2$ years (95\% UI 5.6-6.6), from 65.3 years (65.0-65.6) in 1990 to $71 \cdot 5$ years (71.0-71.9) in 2013, HALE at birth rose by $5 \cdot 4$ years $(4 \cdot 9-5 \cdot 8)$, from $56 \cdot 9$ years $(54 \cdot 5-59 \cdot 1)$ to 62.3 years $(59 \cdot 7-64 \cdot 8)$, total DALYs

\footnotetext{
Correspondence to: Prof Christopher J L Murray, Institute for Health Metrics and Evaluation, 2301 5th Avenue, Suite 600, Seattle, WA 98121, USA, cjlm@uw.edu.

Contributors

TV, ADL, JAS, and CJLM prepared the first draft. CJLM and TV finalised the draft based on comments from other authors and reviewer feedback. TV, ADL, JAS, and CJLM conceived of the study and provided overall guidance. RB and KF performed final statistical analyses. All other authors provided data, developed models, reviewed results, provided guidance on methodology, and reviewed the manuscript.

${ }^{*}$ Collaborators listed at the end of the Article

For the United Nations Development Programme Human Development Index see http://hdr.undp.org/en/content/human-developmentindex-hdi

See Online for appendix
} 
fell by $3 \cdot 6 \%(0 \cdot 3-7 \cdot 4)$, and age-standardised DALY rates per 100000 people fell by $26 \cdot 7 \%(24 \cdot 6-$ 29.1). For communicable, maternal, neonatal, and nutritional disorders, global DALY numbers, crude rates, and age-standardised rates have all declined between 1990 and 2013, whereas for non-communicable diseases, global DALYs have been increasing, DALY rates have remained nearly constant, and age-standardised DALY rates declined during the same period. From 2005 to 2013, the number of DALYs increased for most specific non-communicable diseases, including cardiovascular diseases and neoplasms, in addition to dengue, food-borne trematodes, and leishmaniasis; DALYs decreased for nearly all other causes. By 2013, the five leading causes of DALYs were ischaemic heart disease, lower respiratory infections, cerebrovascular disease, low back and neck pain, and road injuries. Sociodemographic status explained more than $50 \%$ of the variance between countries and over time for diarrhoea, lower respiratory infections, and other common infectious diseases; maternal disorders; neonatal disorders; nutritional deficiencies; other communicable, maternal, neonatal, and nutritional diseases; musculoskeletal disorders; and other non-communicable diseases. However, sociodemographic status explained less than $10 \%$ of the variance in DALY rates for cardiovascular diseases; chronic respiratory diseases; cirrhosis; diabetes, urogenital, blood, and endocrine diseases; unintentional injuries; and self-harm and interpersonal violence. Predictably, increased sociodemographic status was associated with a shift in burden from YLLs to YLDs, driven by declines in YLLs and increases in YLDs from musculoskeletal disorders, neurological disorders, and mental and substance use disorders. In most country-specific estimates, the increase in life expectancy was greater than that in HALE. Leading causes of DALYs are highly variable across countries.

Interpretation-Global health is improving. Population growth and ageing have driven up numbers of DALYs, but crude rates have remained relatively constant, showing that progress in health does not mean fewer demands on health systems. The notion of an epidemiological transition-in which increasing sociodemographic status brings structured change in disease burden-is useful, but there is tremendous variation in burden of disease that is not associated with sociodemographic status. This further underscores the need for country-specific assessments of DALYs and HALE to appropriately inform health policy decisions and attendant actions.

\section{Introduction}

The Global Burden of Disease study 2013 (GBD 2013) seeks to bring together all available epidemiological data using a coherent measurement framework, standardised estimation methods, and transparent data sources to allow comparisons of health loss to be made over time and across causes, age-sex groups, and geographies. The GBD 2013 data for disease and injury incidence and prevalence, years lived with disability (YLDs), causes of death, and years of life lost because of premature mortality (YLLs) for 188 countries provide an opportunity to assess the effect of recent changes in population health by examining summary measures of health loss attributed to specific causes, expressed in DALYs, and summary measures of average population health, expressed as HALE. ${ }^{1,2}$ These measures are crucial to track health progress, strengthen policy decisions, assess programme effects and results, and inform health service and research priorities. Such holistic measures of population health, encompassing both disability and mortality levels and patterns in populations, are also attracting interest as part of the discussion around the Sustainable Development Goals . ${ }^{3-5}$ 
A hallmark of the GBD approach is an emphasis on making national data easier to compare by taking into account the extensive variation that exists in national medical certification and cause of death coding practices and widely varying case definitions and measurement methods used to track the incidence and prevalence of diseases and injuries. ${ }^{1,2}$ The GBD not only provides detailed metrics for specific causes, but also generates summary measures, such as DALYs and HALE, which enable comparative assessments of broad epidemiological patterns across countries and different time periods. HALE is a useful summary of overall health for a country and DALYs allow assessment of both premature mortality and non-fatal outcomes by cause. These broad summary measures allow quantification of general trends, such as the epidemiological transition, while also making clear how countries and regions deviate from general patterns. ${ }^{6-9}$ The unfolding of the HIV epidemic and the rise of adult mortality, especially among men in Eastern Europe and Central Asia, have called into question the notion of a universal pattern of epidemiological change that occurs with sociodemographic development. ${ }^{2,10-13}$ However, the general notion of a shift from communicable to non-communicable causes of disease burden and injuries remains a powerful framework for global and regional health policy debates. ${ }^{9,14-18}$ The GBD provides an opportunity to quantify these patterns and explore the extent to which epidemiological change is driven by sociodemographic change, reduction of health risks, improvement of health management, or other local factors.

GBD 2013 results for deaths, YLLs, incidence, prevalence, and YLDs by cause for 1990 to 2013 for 188 countries have already been published. ${ }^{1,2}$ In this study we use these GBD 2013 results to calculate DALYs and HALE. These summary metrics are used to characterise broad patterns of lost healthy life and cross-country variations within these patterns. The GBD 2013 provides a complete re-analysis of each country's data from 1990 to 2013 and thus supersedes all previously published GBD analyses of DALYs and HALE.

\section{Methods}

\section{Study design}

GBD 2013 uses a hierarchy of causes that organises 306 diseases and injuries into four levels of classification, the rationale for which has been described previously. ${ }^{2,19}$ The first level distinguishes three broad categories: first, communicable, maternal, neonatal, and nutritional disorders; second, non-communicable diseases; and third, injuries. Level 2 has 21 mutually exclusive and collectively exhaustive categories, level 3 has 163 categories, and level 4 has 254 categories. The full cause list, including International Classification of Diseases tenth edition (ICD-10) codes, has been reported previously. ${ }^{1,2}$ Mortality rates and causes of death for each country-age-sex-year group have been estimated in accordance with some general principles: identification of all available data sources, evaluation of the quality and correction for known bias in each data source, consistent statistical estimation including uncertainty analysis, and cross-validation analysis to assess model performance. Details of data sources and estimation methods used to deal with missing data and multiple measurements for the same country-age-sex-year group have been described previously. ${ }^{2}$ Disease and injury incidence and prevalence and computation of YLDs have been estimated in line with similar principles of identification and assessment of the quality of all available 
sources for 2337 sequelae of the 301 diseases and injuries. ${ }^{1}$ The discrepancy between the 306 diseases and injuries for which DALYs are calculated and the 301 diseases and injuries for which YLDs are calculated is attributable to five diseases that cause death but do not cause disability: sudden infant death syndrome, indirect maternal deaths, late maternal deaths, maternal deaths aggravated by HIV/AIDS, and aortic aneurysm. Various statistical estimation methods were used depending on the details of specific diseases, the most common approach being the application of a Bayesian metaregression model, DisMod-MR 2.0. ${ }^{20}$ We used alternative methods when the basic susceptible, with disease, and dead states in DisMod-MR 2.0 were insufficient to capture the natural history of a sequela. We aggregated sequelae prevalence into YLDs first by estimating the distribution of comorbidities through microsimulation, and second by using disability weights derived from population-based surveys of the general public to assign disability weights to each sequela and combination of sequelae-details of both steps have been described previously. 1,21

We used the GBD 2013 results for YLLs ${ }^{2}$ and YLDs ${ }^{1}$ to calculate DALYs. To calculate HALE, we used YLDs per person and life tables. ${ }^{1,2}$ We applied decomposition of variance using hierarchical regression to DALY rates by cause.

\section{Years lived with disability}

For each year for which YLDs have been estimated (1990, 1995, 2000, 2005, 2010, and 2013), we computed DALYs by adding YLLs and YLDs for each age-sex-country group. We assumed that uncertainty in YLLs is independent of uncertainty in YLDs. We did this by summing the first draw of the 1000 draws for YLLs and YLDs and then repeating for each subsequent draw. We calculated $95 \%$ uncertainty intervals (UIs) using the 25 th and 975 th ordered draw of the DALY uncertainty distribution.

\section{Healthy life expectancy}

We calculated HALE in accordance with the methods outlined by Salomon and colleagues. ${ }^{8}$ In brief, we used Sullivan's method ${ }^{22}$ to incorporate information about average levels of health experienced at different ages into an abridged life table to produce estimates of life expectancy that are adjusted for reductions in functional health status relating to prevalent health conditions. Effectively, the cumulative years lived in an age group in the abridged life table (the life expectancy column) for each country-age-sex group is multiplied by the YLDs per person for that country-age-sex group. Calculation of HALE relies on three inputs from GBD 2013: life tables by sex, country, and year; estimates of the prevalence of 2337 sequelae by age, sex, country and year; and disability weights for 235 unique health states that collectively cover the range of functional health losses and symptoms associated with the 2337 sequelae. Wang and colleagues ${ }^{2}$ have described data sources and methods to estimate mortality and life tables, and Vos and colleagues ${ }^{1}$ have described these for the measurement of prevalence of sequelae and disability weights. ${ }^{1}$ We combined information about prevalence and disability weights into measures of the overall rate of YLDs per person in each age-sex-country group. We make the strong assumption that uncertainty in YLDs per person is independent of uncertainty in age-specific death rates to calculate uncertainty distributions for HALE. 


\section{Decomposition of variance and epidemiological transition}

The epidemiological transition is an extension of the notion of the demographic transition.

In demographic transition, a characteristic evolution occurs in populations over time towards reduced fertility rates, reduced mortality rates, and an older age distribution of the population. The widely used concept of the epidemiological transition adds the idea that, in addition to these changes, a characteristic change occurs in the contributing causes of death. The epidemiological transition has been broadened to encompass the more general health transition, including both morbidity and mortality. A single variable to characterise countries over time in terms of their demographic and epidemiological status would be crucial to describe the epidemiological transition. Some studies examine associations with income per person, whereas others use variables such as mean age of the population. ${ }^{23,24}$ We aimed to construct a single composite variable to represent both demographic status and socioeconomic development to explore the patterns of the epidemiological transition. To construct this sociodemographic status variable, we assessed variables indicative of socioeconomic status and demographic change that were available for all 188 countries from 1990 to 2013. We did not include measures of income inequality, such as the Gini coefficient, because these were not available for all countries for each year. We used principal components analysis (PCA) of the log transformation of income per person (in constant international dollars), average years of schooling of the population after age 15 years, the log of the total fertility rate, and the log of the mean age of the population. The relationship between the PCA variables and DALY rates were highly non-linear, but became linear with respect to the log DALY rates after log transformation of three of the four sociodemographic status component variables. Before using PCA, we normalised each variable to have a mean of zero and a standard deviation of 1.0. Only the first component of the PCA had an eigenvalue greater than 1.0 and the weights were 0.471 for income per person, -0.517 for total fertility rate, 0.495 for education per person, and 0.516 for mean age. ${ }^{25}$ As expected, the sign on the total fertility rate was negative, whereas the sign on the other three components was positive. We also tested all possible combinations of the four variables using the same PCA approach to confirm that the principal component of all four was the most predictive of variation in DALY rates by cause. We used the predicted value of the first component for each country-year in the subsequent ANOVA and predictions of the epidemiological change with sociodemographic status.

We used hierarchical regression to decompose variance in log DALY rates into components related to the sociodemographic status, intercountry variation, year, and fraction explained by the interactions of the other variables. This approach estimates a simple model with uncorrelated random effects for year, country, and sociodemographic status. ${ }^{26}$ We divided sociodemographic status into vigintiles (20 equal interval bins) to allow for non-linear correlations between log DALY rates and sociodemographic status for a cause. We did tests with up to 50 bins for the sociodemographic status variable with no change in qualitative results. We divided the variance of each random effect by total variance to decompose variance into different factors. We did this variance decomposition analysis for GBD level 2 and level 3 causes. We use these regressions to predict the pattern of DALYs by cause (and through separate regressions for YLLs and YLDs) purely as a function of sociodemographic status, holding all other random effects (year and country) at zero. Because there could be 
lagged effects between sociodemographic status and DALY rate, we tested alternative models with sociodemographic status lagged from 1 to 10 years. Use of sociodemographic status from the same year as the DALY rates explained, on average, the highest proportion of the variance in DALY rates.

\section{Age standardisation}

We selected the revised GBD 2013 world population standard for the age standardisation of rates. Details of the age standard, and its development, have been reported previously. ${ }^{2}$

\section{Role of the funding source}

The funder of the study had no role in study design, data collection, data analysis, data interpretation, or writing of the report. All authors had access to the data in the study and final responsibility to submit the paper.

\section{Results \\ Global}

Global life expectancy at birth for both sexes combined increased from 65.3 years (95\% UI 65.0-65.6) in 1990 to 71.5 years (71.0-71.9) in 2013, whereas during the same interval, HALE at birth for both sexes combined increased from 56.9 years $(54.5-59 \cdot 1)$ to 62.3 years (59.7-64.8). The survivorship curves shift up and to the right with increasing quintiles of country sociodemographic status (figure 1). In the three groups of countries, defined as the lowest, middle three, and highest quintiles of country sociodemographic status in 2013, individuals are distributed across ranges of disability weights, with the majority of the population in most age groups living in health states with disability weights in the range $0 \cdot 0$ $0 \cdot 1$. The fraction of individuals in the life table in full health (ie, living with a disability weight of zero) is $16.3 \%$ in the lowest sociodemographic status quintile of countries. Even in the most advantageous sociodemographic quintile of countries, the time lived in full health constitutes only a small fraction (17.5\%) of the overall life course. At the other end of the spectrum, the expectation of years lived with disability weights greater than 0.5 is 3.59 years in the lowest quintile and 6.60 years in the highest quintile of countries.

Figure 2 shows the need to understand global epidemiological change in terms of numbers, rates, and age-standardised rates. The number of DALYs caused by communicable, maternal, neonatal, and nutritional disorders has declined steadily from 1.19 billion (95\% UI 1.15 billion to 1.24 billion) in 1990 to 769.3 million ( 725.5 million to 814.9 million) in 2013, whereas DALYs for non-communicable diseases (NCDs) have increased steadily, rising from 1.08 billion ( 0.97 billion to 1.20 billion) to 1.43 billion ( 1.26 billion to 1.61 billion) during the same period (figure 2A). The year of crossover, during which global DALYs for NCDs exceeded those for global communicable, maternal, neonatal, and nutritional causes, was 1994. DALYs due to injuries have remained relatively constant, decreasing slightly from 269.6 million (251.6 million to 286.7 million) to 247.6 million (231.3 million to $265 \cdot 1$ million). Figure 2B shows crude DALY rates per 100000 people for these three broad cause groups, thereby removing the effect of global population growth during the period. The DALY rate for NCDs has remained fairly constant, while substantial 
declines have occurred in DALY rates for communicable, maternal, neonatal, and nutritional disorders $(52 \cdot 2 \%, 50 \cdot 1-54.4)$ and injuries $(32 \cdot 0 \%, 27 \cdot 0-35.9)$. The analysis of agestandardised DALY rates, shows that, after controlling for changes in population size and composition, NCD disease burden worldwide, has continued to decline, falling by $14.5 \%$ (11.6-17.3) between 1990 and 2013 (figure 3C). During the same period, worldwide agestandardised DALY rates fell by $42.4 \%(40 \cdot 0-45 \cdot 0)$ for communicable, maternal, neonatal, and nutritional disorders and $30.9 \%(26 \cdot 1-34 \cdot 7)$ for injuries.

We decomposed the changes in the number of DALYs into trends for two periods: 19902005 and 2005-2013 (figure 3). ${ }^{10}$ Between 1990 and 2005, the number of global DALYs changed only slightly, from 2.54 billion (95\% UI 2.40 billion to 2.70 billion) to 2.51 billion ( 2.33 billion to 2.72 billion). Looking at disease-level details within this relative stagnation reveals important trends for specific diseases (figure $3 \mathrm{~A}$ ); the earlier period was characterised by decreases in the number of DALYs from diarrhoea, lower respiratory infections, measles, neonatal causes, tuberculosis, and tetanus, with smaller contributions from declines in congenital causes and some injuries. Conversely, large increases in disease burden were recorded for HIV/AIDS and malaria, with smaller increases for road injuries and a diverse range of NCDs, including ischaemic heart disease, diabetes, low back and neck pain, stroke, and depression, in addition to several types of cancer. From 2005 to 2013, total DALYs worldwide decreased from 2.51 billion ( 2.33 billion to 2.72 billion) to 2.45 billion (2.23 billion to 2.68 billion; figure 3B). Decreases were recorded for diarrhoea, malaria, HIV/AIDS, lower respiratory infections, measles, tuberculosis, and neonatal causes, and nearly all injuries. Increases were noted for a wide range of NCDs, especially low back and neck pain, ischaemic heart disease, diabetes, chronic obstructive pulmonary disease (COPD), depression, stroke, and sense organ disorders. Although they were not large contributors to the number of DALYs, notable increases were seen for dengue, food-borne trematodes, and leishmaniasis. Separate analyses of changes in age-standardised DALY rates for the period 2005-13 (data not shown) suggest that most of the increases shown in figure 3 are caused by ageing of the population and population growth.

We assessed changes in the age-standardised DALY rates of the leading GBD level 3 causes for 1990-2005 and 2005-2013 (figure 4; level 4 of the GBD cause hierarchy is reported in the appendix p 2). Between 1990 and 2005 huge reductions occurred in measles, meningitis, iron-deficiency anaemia, congenital anomalies, tuberculosis, drowning, protein-energy malnutrition, and some neonatal disorders, whereas disease burden from HIV/AIDS and malaria substantially increased (figure 4). From 2005 to 2013, age-standardised DALY rates for ischaemic heart disease, lower respiratory infections, and cerebrovascular disease have declined, although not sufficiently for these conditions to be replaced as the leading causes of disease burden worldwide. The ranks of low back and neck pain, road injuries and COPD have all increased since 2005. Age-standardised rates decreased significantly for 16 of the 25 leading causes of DALYs in 2013; for the remaining nine causes (Alzheimer's disease, chronic kidney disease, congenital anomalies, depressive disorders, diabetes, low back and neck pain, migraine, neonatal sepsis, and skin diseases), age-standardised rates did not significantly change. 
Table 1 shows DALYs for each cause in 2005 and 2013 and changes in numbers and agestandardised rates of the DALYs (for the same information for 1990 to 2013 see appendix $p$ $4)$.

\section{Decomposition of epidemiological patterns}

We decomposed the variance of DALY rates for GBD level 2 causes into contributions from sociodemographic status, year, country, and unexplained sources (residual; table 2). Sociodemographic status explained more than $50 \%$ of the variance for diarrhoea, lower respiratory infections and other common infectious diseases; maternal disorders; neonatal disorders; nutritional deficiencies; other communicable diseases; musculoskeletal disorders; and other NCDs. Furthermore, sociodemographic status explains between a fifth and a half of the variance for HIV/AIDS and tuberculosis; neurological disorders; mental and substance use disorders; transport injuries; and forces of nature, war, and legal intervention. Sociodemographic status explained little of the variance in the DALY rates for neglected tropical diseases and malaria, for which time-invariant country differences account for $84.98 \%$ of the variance. Notably, less than $10 \%$ of the variance in the burden of several level 2 causes could be related to sociodemographic status, including cardiovascular diseases; chronic respiratory diseases; cirrhosis; diabetes, urogenital, blood, and endocrine diseases; unintentional injuries; and self-harm and interpersonal violence. Year explained less than 7\% of variance for all causes. By contrast, time invariant intercountry variation was an important component of the variance in DALY rates for all causes, ranging from a low of $11.29 \%$ for neonatal disorders to $91.23 \%$ for self-harm and interpersonal violence. Intercountry variation explains more than two-thirds of the total variance in DALY rates for HIV/AIDS and tuberculosis; neglected tropical diseases and malaria; neoplasms; cardiovascular diseases; chronic respiratory diseases; cirrhosis; digestive diseases; mental and substance use disorders; diabetes, urogenital, blood, and endocrine disorders; unintentional injuries; and self-harm and interpersonal violence. Notably, together, sociodemographic status and country account for more than $90 \%$ of the variance for 17 of 21 GBD level 2 causes; indeed, the lowest fraction of variance accounted for by these three factors is $80.2 \%$ for forces of nature, war, and legal intervention.

Figure 5 shows the predicted global composition of YLLs and YLDs for level 2 causes at different levels of sociodemographic status, controlling for year and country. YLLs from diarrhoea, lower respiratory infections, and other common infections, and neonatal disorders fall substantially as sociodemographic status increases. Other YLLs that fall noticeably with rising sociodemographic status include YLLs from maternal causes, nutritional deficiencies, other non-communicable causes (including congenital causes), and unintentional injuries. YLLs from cardiovascular diseases at first increase slightly with increasing sociodemographic status, but then decrease at the highest levels of country sociodemographic status. Some important causes of global YLLs are not strongly related to sociodemographic status because they are largely country-specific, such as neglected tropical diseases and malaria, neoplasms, and intentional injuries. By contrast, overall YLDs decline slightly at first, but then increase substantially, showing the opposite trend to YLLs. The large increases in YLDs are related to musculoskeletal disorders; mental and substance use disorders; diabetes, urogenital, blood, and endocrine diseases; and neurological 
disorders. As sociodemographic status rises, the steady decreases in YLLs and increases in YLDs cause the proportion of total DALYs attributable to YLDs to steadily rise from 9.9\% at the lowest level of sociodemographic status to $49.1 \%$ in the highest vigintile. Above the tenth vigintile of sociodemographic status, the rise in YLDs and fall in YLLs nearly compensate for each other so that DALY rates have remained largely constant.

\section{Country-specific results}

In 1990, life expectancy ranged from 46.9 years (95\% UI 45.1-48.2) in the Central African Republic to 80.7 years (78.7-82.5) in Andorra, while HALE ranged from 40.4 years (38.2$42.5)$ in the Central African Republic to 70.2 years (67.7-72.5) in Japan. By 2013, life expectancy ranged from $48 \cdot 3$ years $(46 \cdot 5-50 \cdot 1)$ in Lesotho to 83.9 years $(82 \cdot 3-85 \cdot 5)$ in Andorra, and HALE ranged from 42.0 years (39.8-44.2) in Lesotho to 73.4 years (70.576.0) in Japan. Disaggregating by sex, in 1990, there were no countries in which men had attained a HALE of 70 years or more, and only in Japan and Andorra did women attain this. By 2013, HALE for men exceeded 70 years in only two countries (Japan and Singapore), whereas HALE for women exceeded 70 years in 40 countries (table 3). Of the $21 \mathrm{GBD}$ regions, nine contained at least one country in which female HALE was at least 70 years in 2013. For most countries, changes in HALE were positive for both men and women between 1990 and 2013, and the differences were significant. However, HALE in 2013 was not significantly higher than it was in 1990 in 43 countries for men and in 32 countries for women. As life expectancy increases, the gap between life expectancy and HALE widens, increasing to more than 10 years at a life expectancy of 77 years for women and 78 years for men. The life expectancy minus HALE divided by life expectancy (the percentage of life expectancy lost because of poor health) ranged between $11.5 \%$ and $15.0 \%$.

Figure 6 shows the ten most common causes of DALYs for each country in 2013. The leading causes of DALYs vary substantially across regions, representing both different levels of sociodemographic status and distinct regional patterns. In high-income regions, low back and neck pain, ischaemic heart disease, cerebrovascular disease, and tracheal, bronchus, and lung cancer are often among the four most common causes, although major depression, COPD, and diabetes come into the top four slots in some countries. In central and eastern Europe, cardiovascular diseases rank consistently in the most common causes of DALYs. Self-harm and depression frequently rank higher in eastern Europe than in central Europe or elsewhere. In central Asia, representative of the mixed levels of sociodemographic status present in the region, leading causes include neonatal encephalopathy and congenital causes. In central Latin America, violence, ischaemic heart disease, diabetes, low back and neck pain, and road injuries comprise the top five causes. Other examples of distinct regional patterns include the high ranking of COPD in east Asia, the dominance of malaria in west Africa, and the dominance of HIV/AIDS in eastern and southern sub-Saharan Africa. Within some regions, such as north Africa and the Middle East, the leading causes varied substantially. 


\section{Discussion}

Global health is improving: life expectancy at birth rose by $6 \cdot 2$ years between 1990 and 2013, while HALE at birth increased by 5.4 years during the same interval; worldwide, agestandardised DALY rates fell by $27 \%$. Global progress has accelerated since 2005 because of major reductions in HIV/AIDS and malaria, in addition to continued progress against other major communicable, maternal, neonatal, and nutritional disorders. Although the total volume of DALYs is down by only $3.6 \%$ over the 23 year period, this is largely explained by population growth and ageing driving up numbers of DALYs. Declines in age-standardised DALY rates are counterbalanced by population growth and ageing, so that, despite improvements in age-sex-specific health status, demands on health systems remain high. An example of these demands is the fact that the number of individuals in the world living in states of health characterised by a disability weight greater than 0.1 has increased by $43 \%$ from 1990 to 2013.

In 1971, Omran ${ }^{27}$ outlined the concept of the epidemiological transition to describe the changing pattern of causes of death that results from sociodemographic development. The notion of the epidemiological transition has been expanded to recognise the phase in transition that leads to double burden of disease ${ }^{9,28,29}$ and the countertransitions of the HIV/ AIDS epidemic and the rise of mortality in the former Soviet Union. ${ }^{2,10,11,13,30-32}$ Many studies have recognised regional and national variation in the epidemiological transition. ${ }^{9,14,33-35}$ Taking advantage of the database of the GBD 2013 country-level results from 1990 to 2013, we have quantified the extent to which sociodemographic status accounts for changes in epidemiological patterns, as opposed to other factors changing over time or static variation between countries. Isolation of this component of the variation of DALY rates allows examination of the shifts in disease and injury patterns that would be expected purely as a function of changing sociodemographic status. As sociodemographic status rises, YLLs from diarrhoea, lower respiratory infections, neonatal causes, maternal mortality, and other infectious causes decline substantially, while at the same time, there has been a slower rise in YLDs from musculoskeletal disorders, mental and substance use disorders, neurological disorders, and diabetes, urogenital, blood, and endocrine diseases. DALY rates for neoplasms and cardiovascular disease are minimally related to sociodemographic status; instead local factors have a profound effect. However, with rising sociodemographic status, the proportion of DALYs due to these causes increases because of decreases in other causes of YLLs. Although, DALY rates for cardiovascular disease seem not to be related to sociodemographic status, large declines have been recorded for these causes in high-income countries in age-standardised rates over the past decades. The wide variation between some high sociodemographic status countries, such as Japan and Finland, shows how other factors, such as diet, physical activity, and other risks, vary substantially within the same level of sociodemographic status and also affect cardiovascular disease outcomes. Furthermore, our analysis of the epidemiological transition is based on crude population rates, so reductions in age-specific cardiovascular rates associated with rising sociodemographic status are countered by shifts towards an older population. Our analysis of the epidemiological transition shows decreases in DALY rates for cardiovascular disease at the very highest levels of sociodemographic status. Notably, the predictable rise in YLD 
rates for some causes (such as musculoskeletal disorders, diabetes mellitus, and mental and substance use disorders) driven by population ageing is not well recognised in the literature about the epidemiological transition.

Our decomposition of variance analysis shows that, for many NCDs, the main determinants of variation in DALY rates are country-specific effects, not the epidemiological transition. Global health can be understood in terms of a general theme in which change in epidemiological patterns is related to sociodemographic status, upon which country-specific patterns are overlaid. Little of the variation in DALY rates was attributable to the year, which contrasted with previous findings showing that the association between life expectancy and income and education has shifted over time. ${ }^{24,36-38}$ Our analysis only covers a 23 year period, which might be too short to fully capture the effects of changing sociodemographic status. Some of the country effects, such as those noted for neglected tropical diseases and malaria, might have been related to sociodemographic status in a longer run analysis. The substantial effect of country variation on the epidemiological transition pattern reinforces the importance of estimating the burden of disease for each country individually. The GBD results can be used productively in the future to characterise the deviation of individual countries from the general epidemiological transition. Our analysis of the association between crude DALY rates and country sociodemographic status does not provide insights into within-country disease associations across individual levels of socioeconomic status. For example, findings from multiple studies have shown that individuals of lower socioeconomic status have increased rates of cardiovascular and circulatory diseases. ${ }^{39-47}$

HALE varies widely between countries and over time. As a single summary measure of population health, HALE is fairly simple to explain and provides an indicator that is affected by any changes in mortality rates or prevalence of disease or injury. HALE has been proposed as an indicator for the Sustainable Development Goals. As calculated through the GBD, HALE is an attractive measure that is sensitive to intervention and comparable over time and between populations. Although HALE needs input about the prevalence of multiple sequelae, the annual revisions of the GBD provide a widely available source for regular updates. By contrast, some other variants of health expectancies might be less appropriate for intertemporal or cross-country comparison. With measures that define disability according to arbitrary thresholds of disability weight, such as disability-free life expectancy (DFLE) ${ }^{48}$ even slight changes in the disability weight threshold will profoundly affect conclusions about levels and changes in healthy life expectancy, as can be seen in figure 1 . Moreover, these measures are non-standardised and hence not comparable: in some implementations of DFLE, the choice of the severity threshold to define disability is left to individual respondents in the surveys. For example, the European Union ${ }^{49}$ has adopted a measure of healthy life expectancy based on survey responses to a single item on activity limitations. Such a measure is susceptible to variation in the meaning attached to categorical descriptions of limitation levels, both between individuals and over time, as seen in related survey items on health status. ${ }^{50-52}$ An example of the sensitivity of DFLE measured in Europe is the reported decline in DFLE in Italy after 2004, which was caused by a change in question phrasing; we noted an increase in HALE for Italy in this study. ${ }^{53}$ Although HALE and DFLE both use disability weights, the continuous scale of disability weights used in 
HALE makes it less sensitive to measurement error than are the dichotomous (zero or one) weights used in DFLE.

Our findings support those of Salomon and colleagues, ${ }^{8}$ which showed that HALE is increasing more slowly than life expectancy: ie, as life expectancy increases, the expectation of years lived with multiple sequelae increases as well. The difference between life expectancy and HALE has increased, whereas the ratio of this difference to life expectancy has remained fairly constant. Whether or not this change should be termed an expansion of morbidity is not the issue. Rather, we saw that while health loss because of YLDs from cardiovascular and circulatory diseases and neoplasms might be decreasing, the real drivers of the difference between HALE and life expectancy are musculoskeletal disorders, mental and substance use disorders, neurological disorders, and diabetes, along with vision loss and hearing loss. Prevalence for all of these conditions is strongly age-related, and agestandardised rates for them are not declining. Even though the age of onset for mental health and substance use disorders is not strongly age-related, the prevalence of these conditions tends to rise with age. Prevalence of musculoskeletal disorders, neurological disorders, diabetes, and hearing and vision loss rise even more profoundly with age. As individuals increasingly survive to 80 years and older, the amount of time spent with a combination of these disorders increases, even though age-standardised rates have not increased over time. According to our analysis, the available therapies have not led to significant declines in agestandardised YLD rates. Very few, if any, of these disorders receive the attention they deserve in public policy discourse about health and health research priorities.

Global health progress has been driven by impressive progress in reducing age-standardised rates for a wide range of causes of death. Age-standardised YLD rates, however, are not declining. Many potential reasons exist to explain the general success for mortality and absence of success for disease prevalence. Research and development investments by funders such as the US National Institutes of Health (NIH) and the pharmaceutical industry have focused on cardiovascular diseases, neoplasms, and endocrine disorders. ${ }^{54-59}$ As we report, in the early phases of the epidemiological transition, the major driver of change in disease and injury patterns is progress in the reduction of YLLs. We believe that the historical focus of health research funding on causes of YLLs was probably appropriate. However, health progress now means that more research investment is needed for the disorders that debilitate, rather than kill. With each passing year, the shift towards YLDs as the leading causes of disease burden will be more evident. Action is needed now to develop preventive, curative, and ameliorative strategies for these conditions rather than waiting until this shift is even more obvious.

Controlling for sociodemographic status, substantial variation exists for DALY rates between countries. In our decomposition of variance, the importance of intercountry variation fluctuated by cause; for example, country level variation explains little variation for neonatal causes, but most variation for self-harm and interpersonal violence. These findings raise the question of whether the division of countries into 21 regions in GBD based on geographic contiguity and the levels and patterns of adult and child mortality rates is the best way to explain the variance in DALY rates. With country-specific results now available, more sophisticated clustering methods could be used, with various constraints, to propose a 
more empirically calculated set of regions. Regions have two dimensions: analytical and presentational. The presentational dimension is easily addressed because results for any set of countries can easily be generated from the country-specific results. In fact, the GBD Compare data visualisation tool provides several alternative presentational groupings, such as WHO political regions or World Bank regions. However, regions have an analytical effect on the results if the super-region and region hierarchy have been used in the Bayesian modelling. In the GBD cause of death analysis, spatiotemporal Gaussian process regression models use the GBD hierarchy to borrow strength. In DisMod-MR 2.0, the regional hierarchy also affects estimation of the prior for each country analysis. More simply, where data are sparse or not available, the GBD regional groupings can have an important analytical effect. More research is needed on two fronts. First, to explore the extent to which alternative regional groupings would explain more of the variance in the DALY results (or any other GBD indicator). Second, whether analytical tools, such as cause of death ensemble modelling (CODEm $)^{60}$ or DisMod-MR $2.0,{ }^{1}$ could be modified to easily allow for different regional hierarchies for different causes.

This study has all the limitations previously reported for the GBD 2013 analysis of YLLs and YLDs. ${ }^{1,2}$ Additionally, a key limitation is the assumption that uncertainty is independent for YLLs and YLDs. In fact, for diseases modelled with DisMod-MR 2.0, we estimated a correlation between the uncertainty in condition-specific mortality and the uncertainty in prevalence. However, DisMod-MR 2.0 estimates excess mortality related to a cause, not the mortality that would be assigned to a cause according to the ICD principles of underlying cause. In future iterations of the GBD, it might be useful to attempt to use the correlation structure produced from DisMod-MR 2.0 as a proxy for the correlation between the underlying cause and prevalence. By assuming independence, we might be underestimating overall uncertainty in DALYs. However, more careful examination of the uncertainty in YLDs reveals that most uncertainty stems from uncertainty in disability weights and not from uncertainty in prevalence. We have no reason to assume that the uncertainty associated with valuations of health states in population surveys would be correlated with either disease or death outcomes. Inclusion of the smaller component of the correlation of prevalence with mortality in the estimation of DALY uncertainty would probably not substantially alter the large uncertainty already recorded for DALYs. Another key limitation, is that our assessment of the burden of disease related to sociodemographic status was affected by the choice of variables that we have included in our sociodemographic status indicator (income per person, average years of schooling after age 15 years, total fertility rate, and mean age of the population). Alternative measures of sociodemographic status could be developed that might explain more of the variance seen for some GBD level 2 causes of DALYs. However, we experimented with alternative formulations, including the addition of urbanisation and all combinations of the four variables in our index, and established that the approach we used had the most explanatory power. Other variables that would be interesting to include in a composite country-level sociodemographic status measure, such as the Gini coefficient, were not available for all countries in all years. Notably, where both measures are available, the correlation between the sociodemographic status indicator and the UN Human Development Index during the 23 year time period was 0.95 . 
In the post-Millennium Development Goal (MDG) era, there is much interest in identification of appropriate goals for population health and how these goals should be monitored. ${ }^{4}$ Increasingly, measurement frameworks to assess levels of health in populations have moved away from measures of survival to encompass more holistic measures of disability as well as mortality. Our findings suggest that this broader focus is probably going to be increasingly relevant to guide countries' public policy interventions. Large, impressive, and sustained gains are being made against the majority of leading causes of death in most countries, but as our findings show, these gains are not being accompanied by commensurate declines in age-standardised rates of disability, especially from major musculoskeletal disorders, mental and substance use disorders, neurological disorders, and diabetes. Moreover, the failure of health information systems to reliably describe trends in these disorders not only severely hampers policy responses, but contributes greatly to their further neglect and insufficient awareness of the significant part they now play in overall population health. Despite the important country variation in measures of population, now is the time for the global health community, donors and countries alike, to maximise the opportunities for health that have resulted from the data revolution, and ensure that priority is given to the development of scientifically valid, feasible, and informative data systems to measure progress in reducing disability. Improved data and monitoring can help decision makers to reduce DALYs from a wide range of causes by pursuing the most important opportunities for prevention, treatment, and rehabilitation.

\section{Supplementary Material}

Refer to Web version on PubMed Central for supplementary material.

\section{Acknowledgments}

On behalf of the GBD 2010 Genitourinary Diseases Expert Group, GR, NoP, and BB would like to acknowledge that their activities within the GBD 2013 have been made on the behalf of the International Society of Nephrology (ISN). ATA received institutional support and grants from the Graduate School of Medical Sciences, University Medical Center Groningen (UMCG). JM has received support from the National Health and Medical Research Council. LLY is also supported by the National Natural Sciences Foundation of China. UOM would like to acknowledge his funding by the German National Cohort Consortium. RB was provided funding support by the Brien Holden Vision Institute. INA would like to acknowledge her funding support from the National Health and Medical Research Council Public Health (Australian) Early Career Fellowship. KD is supported by a Wellcome Trust Fellowship in Public Health and Tropical Medicine (grant number 099876). The funding sources had no role in the writing of the manuscript or the decision to submit it for publication. JS was supported by the National Natural Science Foundation for Young Scholars of China, (number 81200051); Research Fund for the Doctoral Program of Higher Education of China (number 20110071120060); Science Foundation for Young Scholars in Zhongshan Hospital (number 2012ZSQN04); and the Scientific Project for Fudan University (number 20520133474). RB is funded by an Australian National Health and Medical Research Council Senior Principal Research Fellow. TF is grateful to the Swiss National Science Foundation for an Early and an Advanced Postdoc Mobility fellowship (project numbers PBBSP3-146869 and P300P3-154634). SJY and IHO received funding for their work in a grant from the Korean Health Technology R\&D Project (Ministry of Health \& Welfare, Republic of Korea; grant number HI13C0729). ALT-L was supported by the CGIAR Research Program on Aquatic Agricultural Systems.

Funding Bill \& Melinda Gates Foundation.

\section{GBD 2013 DALYs and HALE Collaborators}

Christopher J L Murray, Ryan M Barber, Kyle J Foreman, Ayse Abbasoglu Ozgoren*, Foad Abd-Allah*, Semaw F Abera*, Victor Aboyans*, Jerry P Abraham*, Ibrahim Abubakar*, 
Laith J Abu-Raddad*, Niveen M Abu-Rmeileh*, Tom Achoki*, Ilana N Ackerman*, Zanfina Ademi*, Arsène K Adou*, José C Adsuar*, Ashkan Afshin*, Emilie E Agardh*, Sayed Saidul Alam*, Deena Alasfoor*, Mohammed I Albittar*, Miguel A Alegretti*, Zewdie A Alemu*, Rafael Alfonso-Cristancho*, Samia Alhabib*, Raghib Ali*, François Alla*, Peter Allebeck*, Mohammad A Almazroa*, Ubai Alsharif*, Elena Alvarez*, Nelson Alvis-Guzman*, Azmeraw T Amare*, Emmanuel A Ameh*, Heresh Amini*, Walid Ammar*, H Ross Anderson*, Benjamin O Anderson*, Carl Abelardo T Antonio*, Palwasha Anwari*, Johan Arnlöv*, Valentina S Arsic Arsenijevic*, Al Artaman*, Rana J Asghar*, Reza Assadi*, Lydia S Atkins*, Marco A Avila*, Baffour Awuah*, Victoria F Bachman*, Alaa Badawi*, Maria C Bahit*, Kalpana Balakrishnan*, Amitava Banerjee*, Suzanne L Barker-Collo*, Simon Barquera*, Lars Barregard*, Lope H Barrero*, Arindam Basu*, Sanjay Basu*, Mohammed O Basulaiman*, Justin Beardsley*, Neeraj Bedi*, Ettore Beghi*, Tolesa Bekele*, Michelle L Bell*, Corina Benjet*, Derrick A Bennett*, Isabela M Bensenor*, Habib Benzian*, Eduardo Bernabé*, Amelia Bertozzi-Villa*, Tariku J Beyene*, Neeraj Bhala*, Ashish Bhalla*, Zulfiqar A Bhutta*, Kelly Bienhoff*, Boris Bikbov*, Stan Biryukov*, Jed D Blore*, Christopher D Blosser*, Fiona M Blyth*, Megan A Bohensky*, Ian W Bolliger*, Berrak Bora Başara*, Natan M Bornstein*, Dipan Bose*, Soufiane Boufous*, Rupert R A Bourne*, Lindsay N Boyers*, Michael Brainin*, Carol E Brayne*, Alexandra Brazinova*, Nicholas J K Breitborde*, Hermann Brenner*, Adam D Briggs*, Peter M Brooks*, Jonathan C Brown*, Traolach S Brugha*, Rachelle Buchbinder*, Geoffrey C Buckle*, Christine M Budke*, Anne Bulchis*, Andrew G Bulloch*, Ismael R Campos-Nonato*, Hélène Carabin*, Jonathan R Carapetis*, Rosario Cárdenas*, David O Carpenter*, Valeria Caso*, Carlos A Castañeda-Orjuela*, Ruben E Castro*, Ferrán CataláLópez*, Fiorella Cavalleri*, Alanur Çavlin*, Vineet K Chadha*, Jung-Chen Chang*, Fiona J Charlson*, Honglei Chen*, Wanqing Chen*, Peggy P Chiang*, Odgerel Chimed-Ochir*, Rajiv Chowdhury*, Hanne Christensen*, Costas A Christophi*, Massimo Cirillo*, Matthew M Coates*, Luc E Coffeng*, Megan S Coggeshall*, Valentina Colistro*, Samantha M Colquhoun*, Graham S Cooke*, Cyrus Cooper*, Leslie T Cooper*, Luis M Coppola*, Monica Cortinovis*, Michael H Criqui*, John A Crump*, Lucia Cuevas-Nasu*, Hadi Danawi*, Lalit Dandona*, Rakhi Dandona*, Emily Dansereau*, Paul I Dargan*, Gail Davey*, Adrian Davis*, Dragos V Davitoiu*, Anand Dayama*, Diego De Leo*, Louisa Degenhardt*, Borja Del Pozo-Cruz*, Robert P Dellavalle*, Kebede Deribe*, Sarah Derrett*, Don C Des Jarlais*, Muluken Dessalegn*, Samath D Dharmaratne*, Mukesh K Dherani*, Cesar Diaz-Torné*, Daniel Dicker*, Eric L Ding*, Klara Dokova*, E Ray Dorsey*, Tim R Driscoll*, Leilei Duan,* Herbert C Duber*, Beth E Ebel*, Karen M Edmond*, Yousef M Elshrek*, Matthias Endres*, Sergey P Ermakov*, Holly E Erskine*, Babak Eshrati*, Alireza Esteghamati*, Kara Estep*, Emerito Jose A Faraon*, Farshad Farzadfar*, Derek F Fay*, Valery L Feigin*, David T Felson*, Seyed-Mohammad Fereshtehnejad*, Jefferson G Fernandes*, Alize J Ferrari*, Christina Fitzmaurice*, Abraham D Flaxman*, Thomas D Fleming*, Nataliya Foigt*, Mohammad H Forouzanfar*, F Gerry R Fowkes*, Urbano Fra.Paleo*, Richard C Franklin*, Thomas Fürst*, Belinda Gabbe*, Lynne Gaffikin*, Fortuné G Gankpé*, Johanna M Geleijnse*, Bradford D Gessner*, Peter Gething*, Katherine B Gibney*, Maurice Giroud*, Giorgia Giussani*, Hector Gomez Dantes*, Philimon Gona*, Diego González-Medina*, Richard A Gosselin*, Carolyn C Gotay*, Atsushi Goto*, Hebe N Gouda*, Nicholas Graetz*, Harish C Gugnani*, Rahul Gupta*, Rajeev Gupta*, Reyna A 
Gutiérrez*, Juanita Haagsma*, Nima Hafezi-Nejad*, Holly Hagan*, Yara A Halasa*, Randah R Hamadeh*, Hannah Hamavid*, Mouhanad Hammami*, Jamie Hancock*, Graeme J Hankey*, Gillian M Hansen*, Yuantao Hao*, Hilda L Harb*, Josep Maria Haro*, Rasmus Havmoeller*, Simon I Hay*, Roderick J Hay*, Ileana B Heredia-Pi*, Kyle R Heuton*, Pouria Heydarpour*, Hideki Higashi*, Martha Hijar*, Hans W Hoek*, Howard J Hoffman*, H Dean Hosgood*, Mazeda Hossain*, Peter J Hotez*, Damian G Hoy*, Mohamed Hsairi*, Guoqing Hu*, Cheng Huang*, John J Huang*, Abdullatif Husseini*, Chantal Huynh*, Marissa L Iannarone*, Kim M Iburg*, Kaire Innos*, Manami Inoue*, Farhad Islami*, Kathryn H Jacobsen*, Deborah L Jarvis*, Simerjot K Jassal*, Sun Ha Jee*, Panniyammakal Jeemon*, Paul N Jensen*, Vivekanand Jha*, Guohong Jiang*, Ying Jiang*, Jost B Jonas*, Knud Juel*, Haidong Kan*, André Karch*, Corine K Karema*, Chante Karimkhani*, Ganesan Karthikeyan*, Nicholas J Kassebaum*, Anil Kaul*, Norito Kawakami*, Konstantin Kazanjan*, Andrew H Kemp*, Andre P Kengne*, Andre Keren*, Yousef S Khader*, Shams Eldin A Khalifa*, Ejaz A Khan*, Gulfaraz Khan*, Young-Ho Khang*, Christian Kieling*, Daniel Kim*, Sungroul Kim*, Yunjin Kim*, Yohannes Kinfu*, Jonas M Kinge*, Miia Kivipelto*, Luke D Knibbs*, Ann Kristin Knudsen*, Yoshihiro Kokubo*, Soewarta Kosen*, Sanjay Krishnaswami*, Barthelemy Kuate Defo*, Burcu Kucuk Bicer*, Ernst J Kuipers*, Chanda Kulkarni*, Veena S Kulkarni*, G Anil Kumar*, Hmwe H Kyu*, Taavi Lai*, Ratilal Lalloo*, Tea Lallukka*, Hilton Lam*, Qing Lan*, Van C Lansingh*, Anders Larsson*, Alicia E B Lawrynowicz*, Janet L Leasher*, James Leigh*, Ricky Leung*, Carly E Levitz*, Bin Li*, Yichong Li*, Yongmei Li*, Stephen S Lim*, Maggie Lind*, Steven E Lipshultz*, Shiwei Liu*, Yang Liu*, Belinda K Lloyd*, Katherine T Lofgren*, Giancarlo Logroscino*, Katharine J Looker*, Joannie Lortet-Tieulent*, Paulo A Lotufo*, Rafael Lozano*, Robyn M Lucas*, Raimundas Lunevicius*, Ronan A Lyons*, Stefan Ma*, Michael F Macintyre*, Mark T Mackay*, Marek Majdan*, Reza Malekzadeh*, Wagner Marcenes*, David J Margolis*, Christopher Margono*, Melvin B Marzan*, Joseph R Masci*, Mohammad T Mashal*, Richard Matzopoulos*, Bongani M Mayosi*, Tasara T Mazorodze*, Neil W Mcgill*, John J Mcgrath*, Martin Mckee*, Abigail Mclain*, Peter A Meaney*, Catalina Medina*, Man Mohan Mehndiratta*, Wubegzier Mekonnen*, Yohannes A Melaku*, Michele Meltzer*, Ziad A Memish*, George A Mensah*, Atte Meretoja*, Francis A Mhimbira*, Renata Micha*, Ted R Miller*, Edward J Mills*, Philip B Mitchell*, Charles N Mock*, Norlinah Mohamed Ibrahim*, Karzan A Mohammad*, Ali H Mokdad*, Glen L D Mola*, Lorenzo Monasta*, Julio C Montañez Hernandez*, Marcella Montico*, Thomas J Montine*, Meghan D Mooney*, Ami R Moore*, Maziar Moradi-Lakeh*, Andrew E Moran*, Rintaro Mori*, Joanna Moschandreas*, Wilkister N Moturi*, Madeline L Moyer*, Dariush Mozaffarian*, William T Msemburi*, Ulrich O Mueller*, Mitsuru Mukaigawara*, Erin C Mullany*, Michele E Murdoch*, Joseph Murray*, Kinnari S Murthy*, Mohsen Naghavi*, Aliya Naheed*, Kovin S Naidoo*, Luigi Naldi*, Devina Nand*, Vinay Nangia*, K M Venkat Narayan*, Chakib Nejjari*, Sudan P Neupane*, Charles R Newton*, Marie Ng*, Frida N Ngalesoni*, Grant Nguyen*, Muhammad I Nisar*, Sandra Nolte*, Ole F Norheim*, Rosana E Norman*, Bo Norrving*, Luke Nyakarahuka*, In-Hwan Oh*, Takayoshi Ohkubo*, Summer L Ohno*, Bolajoko O Olusanya*, John Nelson Opio*, Katrina Ortblad*, Alberto Ortiz*, Amanda W Pain*, Jeyaraj D Pandian*, Carlo Irwin A Panelo*, Christina Papachristou*, Eun-Kee Park*, Jae-Hyun Park*, Scott B Patten*, George C Patton*, Vinod K Paul*, Boris I Pavlin*, Neil Pearce*, David M Pereira*, Rogelio Perez- 
Padilla*, Fernando Perez-Ruiz*, Norberto Perico*, Aslam Pervaiz*, Konrad Pesudovs*, Carrie B Peterson*, Max Petzold*, Michael R Phillips*, Bryan K Phillips*, David E Phillips*, Frédéric B Piel*, Dietrich Plass*, Dan Poenaru*, Suzanne Polinder*, Daniel Pope*, Svetlana Popova*, Richie G Poulton*, Farshad Pourmalek*, Dorairaj Prabhakaran*, Noela M Prasad*, Rachel L Pullan*, Dima M Qato*, D Alex Quistberg*, Anwar Rafay*, Kazem Rahimi*, Sajjad U Rahman*, Murugesan Raju*, Saleem M Rana*, Homie Razavi*, K Srinath Reddy*, Amany Refaat*, Giuseppe Remuzzi*, Serge Resnikoff*, Antonio L Ribeiro*, Lee Richardson*, Jan Hendrik Richardus*, D Allen Roberts*, David RojasRueda*, Luca Ronfani*, Gregory A Roth*, Dietrich Rothenbacher*, David H Rothstein*, Jane T Rowley*, Nobhojit Roy*, George M Ruhago*, Mohammad Y Saeedi*, Sukanta Saha*, Mohammad Ali Sahraian*, Uchechukwu K A Sampson*, Juan R Sanabria*, Logan Sandar*, Itamar S Santos*, Maheswar Satpathy*, Monika Sawhney*, Peter Scarborough*, Ione J Schneider*, Ben Schöttker*, Austin E Schumacher*, David C Schwebel*, James G Scott*, Soraya Seedat*, Sadaf G Sepanlou*, Peter T Serina*, Edson E Servan-Mori*, Katya A Shackelford*, Amira Shaheen*, Saeid Shahraz*, Teresa Shamah Levy*, Siyi Shangguan*, Jun She*, Sara Sheikhbahaei*, Peilin Shi*, Kenji Shibuya*, Yukito Shinohara*, Rahman Shiri*, Kawkab Shishani*, Ivy Shiue*, Mark G Shrime*, Inga D Sigfusdottir*, Donald H Silberberg*, Edgar P Simard*, Shireen Sindi*, Abhishek Singh*, Jasvinder A Singh*, Lavanya Singh*, Vegard Skirbekk*, Erica Leigh Slepak*, Karen Sliwa*, Samir Soneji*, Kjetil Søreide*, Sergey Soshnikov*, Luciano A Sposato*, Chandrashekhar T Sreeramareddy*, Jeffrey D Stanaway*, Vasiliki Stathopoulou*, Dan J Stein*, Murray B Stein*, Caitlyn Steiner*, Timothy J Steiner*, Antony Stevens*, Andrea Stewart*, Lars J Stovner*, Konstantinos Stroumpoulis*, Bruno F Sunguya*, Soumya Swaminathan*, Mamta Swaroop*, Bryan L Sykes*, Karen M Tabb*, Ken Takahashi*, Nikhil Tandon*, David Tanne*, Marcel Tanner*, Mohammad Tavakkoli*, Hugh R Taylor*, Braden J Te Ao*, Fabrizio Tediosi*, Awoke M Temesgen*, Tara Templin*, Margreet Ten Have*, Eric Y Tenkorang*, Abdullah S Terkawi*, Blake Thomson*, Andrew L Thorne-Lyman*, Amanda G Thrift*, George D Thurston*, Taavi Tillmann*, Marcello Tonelli*, Fotis Topouzis*, Hideaki Toyoshima*, Jefferson Traebert*, Bach X Tran*, Matias Trillini*, Thomas Truelsen*, Miltiadis Tsilimbaris*, Emin M Tuzcu*, Uche S Uchendu*, Kingsley N Ukwaja*, Eduardo A Undurraga*, Selen B Uzun*, Wim H Van Brakel*, Steven Van De Vijver*, Coen H van Gool*, Jim Van Os*, Tommi J Vasankari*, N Venketasubramanian*, Francesco S Violante*, Vasiliy V Vlassov*, Stein Emil Vollset*, Gregory R Wagner*, Joseph Wagner*, Stephen G Waller*, Xia Wan*, Haidong Wang*, Jianli Wang*, Linhong Wang*, Tati S Warouw*, Scott Weichenthal*, Elisabete Weiderpass*, Robert G Weintraub*, Wang Wenzhi*, Andrea Werdecker*, Ronny Westerman*, Harvey A Whiteford*, James D Wilkinson*, Thomas N Williams*, Charles D Wolfe*, Timothy M Wolock*, Anthony D Woolf*, Sarah Wulf*, Brittany Wurtz*, Gelin Xu*, Lijing L Yan*, Yuichiro Yano*, Pengpeng Ye*, Gökalp K Yentür*, Paul Yip*, Naohiro Yonemoto*, Seok-Jun Yoon*, Mustafa Z Younis*, Chuanhua Yu*, Maysaa E Zaki*, Yong Zhao*, Yingfeng Zheng*, David Zonies*, Xiaonong Zou*, Joshua A Salomon $\dagger$, Alan D Lopez $\dagger$, Theo Vos $\dagger$. *Authors listed alphabetically. †Joint senior authors. 


\section{Affiliations}

Institute for Health Metrics and Evaluation (Prof C J L Murray D Phil, R M Barber BS, K J Foreman MPH, T Achoki MD, A Afshin MD, V F Bachman BA, A Bertozzi-Villa BA, K BienhoffMA, S Biryukov BS, J D Blore PhD, I W Bolliger BA, J C Brown MAIS, A Bulchis MPH, M M Coates BS, L E Coffeng PhD, M S Coggeshall BA, Prof L Dandona PhD, E Dansereau BA, D Dicker BS, H C Duber MD, K Estep MPA, C Fitzmaurice MD, A D Flaxman PhD, T D Fleming BS, Prof M H Forouzanfar PhD, D González-Medina BA, N Graetz BS, J Haagsma PhD, H Hamavid BA, J Hancock MLS, G M Hansen MSW, Prof S I Hay DSc, K R Heuton BChe, H Higashi PhD, C Huynh BA, M L Iannarone MSc, N J Kassebaum MD, H H Kyu PhD, C E Levitz MPH, S S Lim PhD, M Lind BS, K T Lofgren MPH, R Lozano PhD, M F MacIntyre MEd, C Margono BS, A McLain MA, A H Mokdad PhD, M D Mooney BS, M Moradi-Lakeh PhD, M L Moyer BS, W T Msemburi MPhil, E C Mullany BA, Prof M Naghavi PhD, M Ng PhD, G Nguyen BA, S L Ohno BA, K Ortblad MPH, A W Pain MPH, B K Phillips BA, D E Phillips BS, L Richardson BS, D A Roberts BS, G A Roth MD, L Sandar BS, A E Schumacher BS, P T Serina MPH, K A Shackelford BA, L Singh BS, E Slepak MLIS, J D Stanaway PhD, C Steiner MPH, A Stevens PhD, A Stewart MPH, A M Temesgen PhD, T Templin BA, B Thomson BA, T Vos PhD, J Wagner BS, X Wan PhD, H Wang PhD, T M Wolock BA, S Wulf MPH, B Wurtz MPH), Harborview Injury Prevention and Research Center (B E Ebel MD), Seattle Children's Hospital (N J Kassebaum MD) University of Washington, Seattle, WA, USA (R Alfonso-Cristancho PhD, Prof B O Anderson MD, C D Blosser MD, P N Jensen PhD, C N Mock PhD, T J Montine $\mathrm{PhD}$, D Quistberg PhD); Department of Infectious Disease Epidemiology (T Fürst PhD), Imperial College London, London, UK (G S Cooke DPhil, K J Foreman MPH, Prof D L Jarvis MD, Prof T N Williams MD); Institute of Population Studies (A Abbasoglu Ozgoren MSc, A Çavlin PhD), Institute of Public Health (B Kucuk Bicer PhD), Hacettepe University, Ankara, Turkey; Faculty of Medicine, Cairo-University, Cairo, Egypt (Prof F Abd-Allah MD); School of Public Health, College of Health Sciences, Mekelle University, Mekelle, Ethiopia (S F Abera MSc); Kilte Awlaelo-Health and Demographic Surveillance Site, Mekelle, Ethiopia (S F Abera MSc); Dupuytren University Hospital, Limoges, France (Prof V Aboyans PhD); Family Medicine Residency Program at California Hospital, University of Southern California, Los Angeles, CA, USA (J P Abraham MD); Institute for Global Health (J P Abraham MD), Department of Nutrition (A L Thorne-Lyman ScD), School of Public Health (J P Abraham MD, E L Ding ScD, G R Wagner MD), Harvard University, Boston, MA, USA (I R Campos-Nonato PhD, J A Salomon PhD, M G Shrime MD); Department of Epidemiology and Public Health (H Benzian PhD), University College London, London, UK (Prof I Abubakar PhD); Weill Cornell Medical College Ar-Rayyan Qatar, Doha, Qatar (L J Abu-Raddad PhD); Institute of Community and Public Health, Birzeit University, Ramallah, Palestine (N M Abu-Rmeileh PhD); General Practice and Primary Health Care Academic Centre (P P Chiang PhD), Departments of Medicine and the Florey (A Meretoja $\mathrm{PhD}$ ), University of Melbourne, Melbourne, VIC, Australia (I N Ackerman PhD, Z Ademi $\mathrm{PhD}$, J D Blore PhD, M A Bohensky PhD, Prof P M Brooks MD, S M Colquhoun PhD, Prof A D Lopez PhD, Prof G C Patton MD, Prof H R Taylor AC, R G Weintraub MBBS); University of Basel, Basel, Switzerland (Z Ademi PhD, H Amini MSPH), Swiss Tropical and Public Health Institute (Prof M Tanner PhD, F Tediosi PhD), Basel University, Basel, 
Switzerland (Z Ademi PhD); Association Ivoirienne pour le Bien-Être Familial, Abidjan, Côte d'Ivoire (A K Adou MD); University of Extremadura, Cáceres, Spain (Prof J C Adsuar $\mathrm{PhD}$, U-F Paleo PhD); Friedman School of Nutrition Science and Policy (A Afshin MD, R Micha PhD, D Mozaffarian MD), Tufts University, Boston, MA, USA (S Shangguan MD, P Shi PhD); Institution of Public Health Sciences, Stockholm, Sweden (E E Agardh PhD); International Centre for Diarrhoeal Disease Research (ICDDR), Bangladesh, Dhaka, Bangladesh (S Alam MSc, A Naheed PhD); Ministry of Health, Al Khuwair, Oman (D Alasfoor MSc); Independent, Damascus, Syria (M I Albittar BS); Departamento de Medicina Preventiva y Social (M A Alegretti MD), Universidad de la República—Facultad de Medicina, Montevideo, Uruguay (F Cavalleri BS); Debre Markos University, Addis Ababa, Ethiopia (Z A Alemu MPH); King Abdullah Bin Abdulaziz University Hospital, Riyadh, Saudi Arabia (S Alhabib PhD); Department of Zoology (P Gething PhD), University of Oxford, Oxford, UK (R Ali MSc, D A Bennett PhD, A D Briggs MSc, Prof S I Hay DSc, F B Piel PhD, K Rahimi DM, P Scarborough DPhil); Melbourne Health, Parkville, VIC, Australia (K B Gibney FRACP); School of Public Health, University of Lorraine, Nancy, France (Prof F Alla PhD); Department of Public Health Sciences (P Allebeck PhD, N Roy MD), Department of Neurobiology, Care Sciences and Society (NVS) (S Fereshtehnejad MD), Department of Medical Epidemiology and Biostatistics (E Weiderpass PhD), Aging Research Center (Prof M Kivipelto PhD), Karolinska Institute, Stockholm, Sweden (R Havmoeller PhD, S Sindi PhD); Ministry of Health, Riyadh, Saudi Arabia (M A AlMazroa MD, M O Basulaiman PhD, M Y Saeedi PhD); Charité University Medicine Berlin, Berlin, Germany (U Alsharif MPH, Prof M Endres MD); Government, Madrid, Spain (E Alvarez PhD); Universidad de Cartagena, Cartagena de Indias, Colombia (Prof N Alvis-Guzman PhD); Department of Epidemiology (A T Amare MPH), Department of Psychiatry (Prof H W Hoek MD), University Medical Center Groningen, University of Groningen, Groningen, Netherlands; College of Medicine and Health Sciences, Bahir Dar University, Bahir Dar, Ethiopia (A T Amare); Ahmadu Bello University, Zaria, Nigeria (Prof E A Ameh MBBS); Kurdistan Environmental Health Research Center, Kurdistan University of Medical Sciences, Sanandaj, Iran (H Amini MSPH); Ministry of Public Health, Beirut, Lebanon (W Ammar PhD, H L Harb MPH); St George's, University of London, London, UK (Prof H R Anderson MD); Department of Health Policy and Administration, College of Public Health (C T Antonio MD), University of the Philippines Manila, Manila, Philippines (C I A Panelo MD); Self employee, Kabul, Afghanistan (P Anwari MS); Department of Medical Sciences, Uppsala University, Uppsala, Sweden (Prof J Arnlöv PhD); Dalarna University, Falun, Sweden (Prof J Arnlöv PhD); School of Medicine, Institute of Microbiology and Immunology, University of Belgrade, Belgrade, Serbia (Prof V S Arsic Arsenijevic PhD); University Children's Hospital Belgrade, Belgrade, Serbia (Prof V S Arsic Arsenijevic PhD); Consultant, Windsor, ON, Canada (A Artaman PhD); South Asian Public Health Forum, Islamabad, Pakistan (R J Asghar MD); Mashhad University of Medical Sciences, Mashhad, Iran (R Assadi PhD); Wellness, Human Services and Gender Relations, Ministry Of Health, Castries, Saint Lucia (L S Atkins MPH); National Institute of Public Health, Cuernavaca, Mexico (M A Avila BS, S Barquera PhD, I R Campos-Nonato PhD, L Cuevas-Nasu MsC, H Gomez Dantes MC, I Heredia-Pi PhD, R Lozano PhD, C Medina MS, J C Montañez Hernandez MSc, Prof E E Servan-Mori MSc, T Shamah Levy $\mathrm{PhD}$ ); Komfo Anokye Teaching Hospital, Kumasi, Ghana (B Awuah MD); Public Health 
Agency of Canada, Toronto, ON, Canada (A Badawi PhD); INECO Neurociencias, Rosario, Argentina (M C Bahit MD); Sri Ramachandra University, Chennai, India (K Balakrishnan $\mathrm{PhD}$ ); University of Birmingham, Birmingham, UK (A Banerjee DPhil); School of Psychology (S L Barker-Collo PhD), University of Auckland, Auckland, New Zealand (B del Pozo-Cruz PhD); Department of Occupational and Environmental Health, University of Gothenburg, Gothenburg, Sweden (Prof L Barregard MD); Department of Industrial Engineering, Pontificia Universidad Javeriana, Bogota, Colombia (L H Barrero ScD); School of Health Sciences, University of Canterbury, Christchurch, New Zealand (A Basu $\mathrm{PhD}$ ); School of Medicine (L Gaffikin DrPH), Stanford University, Stanford, CA, USA (S Basu PhD); Oxford University, Ho Chi Minh City, Vietnam (J Beardsley MBChB); College of Public Health and Tropical Medicine, Jazan, Saudi Arabia (N Bedi MD); IRCCS Istituto di Ricerche Farmacologiche Mario Negri, Milano, Italy (E Beghi MD, M Cortinovis Biotech D, G Giussani Biol D); Madawalabu University, Bale Goba, Ethiopia (T Bekele MPH); Yale University, New Haven, CT, USA (Prof M L Bell PhD, J J Huang MD); National Institute of Psychiatry Ramon de la Fuente Muñiz, Mexico City, Mexico (C Benjet PhD, R A Gutiérrez $\mathrm{PhD}$ ); University of Sao Paulo, Sao Paulo, Brazil (I M Bensenor PhD, Prof P A Lotufo DrPH, Prof I S Santos PhD, Prof A H Kemp PhD); Department of Epidemiology and Health Promotion, College of Dentistry (H Benzian PhD), Nelson Institute of Environmental Medicine, School of Medicine (Prof G D Thurston ScD), New York University, New York, NY, USA (Prof H Hagan PhD); King's College London, London, UK (E Bernabé PhD, Prof R J Hay MD, Prof C D Wolfe MD); School of Public Health (K Deribe MPH), Addis Ababa University, Addis Ababa, Ethiopia (T J Beyene DVM, W Mekonnen PhD); Queen Elizabeth Hospital Birmingham, Birmingham, UK (N Bhala DPhil); University of Otago Medical School, New Zealand (N Bhala DPhil); Postgraduate Institute of Medical Education and Research, Chandigarh, India (A Bhalla MD, Prof V Jha DM); Medical Center (Z A Bhutta $\mathrm{PhD}$ ), Aga Khan University, Karachi, Pakistan (M I Nisar MSc); A.I.Evdokimov Moscow State University of Medicine and Dentistry, Moscow, Russia (B Bikbov MD); Academician V.I.Shumakov Federal Research Center of Transplantology and Artificial Organs, Moscow, Russia (B Bikbov MD); Sydney School of Public Health (T R Driscoll PhD), University of Sydney, Sydney, NSW, Australia (F M Blyth PhD, Prof A H Kemp PhD, J Leigh PhD); General Directorate of Health Research (B Bora Başara PhD, G K Yentür PhD), Ministry of Health, Ankara, Turkey (S B Uzun MD); Tel Aviv Saurasky Medical Center, Tel Aviv, Israel (Prof N M Bornstein MD); World Bank, Washington, DC, USA (D Bose PhD); Transport and Road Safety (TARS) Research (S Boufous PhD), BHVI (Prof S ResnikoffMD), University of New South Wales, Kensington, NSW, Australia (Prof L Degenhardt PhD, Prof P B Mitchell MD); Anglia Ruskin University, Cambridge, UK (Prof R R A Bourne FRCOphth); Georgetown University, Washington, DC, USA (L N Boyers BA); DanubeUniversity Krems, Krems, Austria (Prof M Brainin PhD); Cambridge Institute of Public Health, Cambridge, UK (Prof C E Brayne MD); Faculty of Health Sciences and Social Work (A Brazinova PhD), Department of Public Health (M Majdan PhD), Trnava University, Trnava, Slovakia; The Ohio State University, Columbus, OH, USA (Prof N J K Breitborde $\mathrm{PhD}$ ); University of Arizona, Tucson, AZ, USA (Prof N J K Breitborde PhD); Division of Clinical Epidemiology and Aging Research (B Schöttker MPH), German Cancer Research Center, Heidelberg, Germany (Prof H Brenner MD); University of Leicester, Leicester, UK (Prof T S Brugha MD); Monash Department of Clinical Epidemiology, Cabrini Institute, 
Melbourne, VIC, Australia (Prof R Buchbinder PhD); Department of Epidemiology and Preventive Medicine, School of Public Health and Preventive Medicine (Prof R Buchbinder $\mathrm{PhD}$ ), Eastern Health Clinical School (B K Lloyd PhD), Monash University, Melbourne, VIC, Australia (Prof B Gabbe PhD, K B Gibney FRACP, Prof A G Thrift PhD); University of California San Francisco, San Francisco, CA, USA (G C Buckle MD, R A Gosselin MD); Texas A\&M University, College Station, TX, USA (C M Budke PhD); University of Calgary, Calgary, AB, Canada (A G Bulloch PhD, Prof S B Patten PhD, Prof M Tonelli MD, J Wang PhD); University of Oklahoma Health Sciences Center, Oklahoma City, OK, USA (H Carabin PhD); Telethon Institute for Child Health Research, Subiaco, WA, Australia (Prof J R Carapetis PhD); Universidad Autonoma Metropolitana, Mexico City, Mexico (R Cárdenas ScD); University at Albany, Rensselaer, NY, USA (Prof D O Carpenter MD); University of Perugia, Perugia, Italy (V Caso MD); Colombian National Health Observatory, Bogota, Colombia (C A Castañeda-Orjuela MS); Epidemiology and Public Health Evaluation Group, Public Health Department, Universidades Nacional de Colombia, Bogota, Colombia (C A Castañeda-Orjuela MS); Universidad Diego Portales, Santiago, Chile (R E Castro PhD); Division of Pharmacoepidemiology and Pharmacovigilance, Spanish

Medicines and Healthcare Products Agency (AEMPS), Ministry of Health, Madrid, Spain (F Catalá-López PhD); Department of Medicine, University of Valencia/CIBERSAM, Valencia, Spain (F Catalá-López PhD); National Tuberculosis Institute, Bengaluru, India (V K Chadha MD); College of Medicine, National Taiwan University, Taipei, Taiwan (Prof J Chang PhD); School of Population Health (D G Hoy PhD), School of Public Health (L D Knibbs PhD), Centre for Clinical Research (J G Scott PhD), University of Queensland, Brisbane, QLD, Australia (F J Charlson MIPH, H E Erskine B PsySc, A J Ferrari B PsySc, H N Gouda PhD, Prof J J McGrath MD, Prof H A Whiteford MD); Division of Intramural Research, National Institute of Environmental Health Sciences, National Institutes of Health, Department of Health and Human Services, Research Triangle Park, NC, USA (H Chen PhD); Department of Health Development (Y Jiang PhD), Institute of Industrial Ecological Sciences (Y Jiang $\mathrm{PhD}$, Prof K Takahashi MD), Department of Environmental Epidemiology (Y Jiang PhD, O Chimed-Ochir MPH, Prof K Takahashi MD) University of Occupational and Environmental Health, Kitakyushu, Japan; University of Cambridge, Cambridge, UK (R Chowdhury PhD, J Murray PhD); Bispebjerg University Hospital, Copenhagen, Denmark (Prof H Christensen DMSCi); Cyprus University of Technology, Limassol, Cyprus (C A Christophi PhD); University of Salerno, Baronissi, Italy (Prof M Cirillo MD); Universidad de la República, Montevideo, Uruguay (V Colistro MSc); Ministerio de Salud Pública, Montevideo, Uruguay (V Colistro); Murdoch Childrens Research Institute, Melbourne, VIC, Australia (S M Colquhoun PhD); MRC Lifecourse Epidemiology Unit, University of Southampton, Southampton, UK (Prof C Cooper FMedSci); University of California, San Diego, La Jolla, CA, USA (M H Criqui MD, S K Jassal MD, M B Stein MD); Mayo Clinic, Rochester, MN, USA (L T Cooper MD); Hospital Municipal Ramon Santamarina, Tandil, Argentina (L M Coppola MD); Centre for International Health, Dunedin School of Medicine (Prof J A Crump MD), Department of Preventive and Social Medicine, Dunedin School of Medicine (S Derrett PhD), University of Otago, Dunedin, New Zealand (Prof R G Poulton PhD); School of Public Health, College of Health, Massey University, Palmerston North, New Zealand (S Derrett PhD); Walden University, Minneapolis, MN, USA (H Danawi PhD); Public Health Foundation of India, New Delhi, India (Prof L Dandona PhD, R Dandona 
PhD, G Kumar PhD, K S Murthy MPH, K Reddy DM [Card]); Guy's and St. Thomas' NHS Foundation Trust, London, UK (P I Dargan FRCP); Wellcome Trust Brighton \& Sussex Centre for Global Health Research, Brighton, UK (Prof G Davey MD); Public Health England, London, UK (A Davis PhD, D F Fay MSc); University of Medicine and Pharmacy Bucharest, Bucharest, Romania (D V Davitoiu PhD); Department of Surgery, Jacobi Medical Center, Atlanta, GA, USA (A Dayama MD); School of Dentistry and Oral Health (Prof R Lalloo PhD), Griffith University, Brisbane, QLD, Australia (Prof D De Leo DSc); US Department of Veterans Affairs, Eastern Colorado Healthcare System, Denver, CO, USA (R P Dellavalle MD); Brighton and Sussex Medical School, Brighton, UK (K Deribe MPH); Mount Sinai Beth Israel, New York, NY, USA (Prof D C Des Jarlais PhD); Icahn School of Medicine at Mount Sinai New York City, NY, USA (Prof D C Des Jarlais PhD); Africa Medical and Research Foundation in Ethiopia, Addis Ababa, Ethiopia (M Dessalegn MPH); University of Peradeniya, Peradeniya, Sri Lanka (S D Dharmaratne MD); School of Medicine (Prof R Lunevicius PhD), The University of Liverpool, Liverpool, UK (M K Dherani PhD, D Pope PhD); Hospital de la Santa Creu i Sant Pau, Barcelona, Spain (C DiazTorné MD); Department of Social Medicine, Faculty of Public Health, Medical UniversityVarna, Varna, Bulgaria (K Dokova PhD); University of Rochester Medical Center, Rochester, NY, USA (Prof E R Dorsey MD); University of Western Australia, Perth, WA, Australia (Prof K M Edmond PHD, Prof G J Hankey MD); Food Science Department, Faculty of Agriculture, University of Tripoli, Tripoli, Libya (Prof Y M Elshrek PhD); The Institute of Social and Economic Studies of Population, Russian Academy of Sciences, Moscow, Russia (Prof S P Ermakov DSc); Federal Research Institute for Health Organization and Informatics, Ministry of Health of Russian Federation, Moscow, Russia (Prof S P Ermakov DSc); Arak University of Medical Sciences and Health Affairs, Arak, Iran (B Eshrati PhD); Non-Communicable Diseases Research Center, Endocrinology and Metabolic Research Institute (Prof A Esteghamati MD, F Farzadfar MD, N Hafezi-Nejad MD, S Sheikhbahaei MD), Multiple Sclerosis Research Center, Neuroscience Institute (P Heydarpour MD, M Sahraian MD), Digestive Diseases Research Institute (S G Sepanlou MD), Tehran University of Medical Sciences, Tehran, Iran; DHPA, UP College of Public Health, Manila, Philippines (E A Faraon MD); National Institute for Stroke and Applied Neurosciences (V L Feigin PhD), Auckland University of Technology, Auckland, New Zealand (B J Te Ao MPH); Boston University, Boston, MA, USA (Prof D T Felson MD); Institute of Education and Sciences, German Hospital Oswaldo Cruz, São Paulo, Brazil (Prof J G Fernandes PhD); Institute of Gerontology, Academy of Medical Science, Kiev, Ukraine (N Foigt PhD); University of Edinburgh, Edinburgh, UK (Prof F R Fowkes PhD); James Cook University, Townsville, QLD, Australia (R C Franklin PhD); Leras Afrique, Cotonou, Benin (F G Gankpé MD); CHU Hassan II, Fez, Morocco (F G Gankpé MD); Division of Human Nutrition, Wageningen University, Wageningen, Netherlands (J M Geleijnse PhD); Agence de Medecine Preventive, Paris, France (B D Gessner MD); University Hospital of Dijon, Dijon, France (Prof M Giroud MD); University of Massachusetts Boston, Boston, MA, USA (Prof P Gona PhD); School of Population and Public Health (F Pourmalek PhD), University of British Columbia, Vancouver, BC, Canada (C C Gotay PhD); Department of Public Health, Tokyo Women's Medical University, Tokyo, Japan (A Goto MD); Saint James School of Medicine, Kralendijk, Netherlands Antilles (Prof H C Gugnani PhD); West Virginia Bureau for Public Health, Charleston, WV, 
USA (R Gupta MD); Fortis Escorts Hospital, Jaipur, India (R Gupta PhD); Brandeis University, Waltham, MA, USA (Y A Halasa MS MA, E A Undurraga PhD); Arabian Gulf University, Manama, Bahrain (Prof R R Hamadeh DPhil); Wayne County Department of Health and Human Services, Detroit, MI, USA (M Hammami MD); School of Public Health (Prof Y Hao PhD), Zhongshan Ophthalmic Center (Y Zheng MD), Sun Yae-Sen University, Guangzhou, China; Parc Sanitari Sant Joan de Déu—CIBERSAM, Sant Boi de Llobregat, Barcelona, Spain (J Haro MD); Universitat de Barcelona, Barcelona, Spain (J Haro MD); International Foundation for Dermatology, London, UK (Prof R J Hay MD); Fundacion Entornos AC, Cuernavaca, Mexico (M Hijar PhD); Department of Epidemiology (Prof H W Hoek MD), Columbia University, New York, NY, USA (A E Moran MD); Epidemiology and Statistics Program, National Institute on Deafness and Other Communication Disorders (H J Hoffman MA), Center for Translation Research and Implementation Science, National Heart, Lung, and Blood Institute (G A Mensah MD), National Heart Lung and Blood Institute (U K A Sampson MD), National Institutes of Health, Bethesda, MD, USA; Albert Einstein College of Medicine, Bronx, NY, USA (Prof H Hosgood PhD); London School of Hygiene \& Tropical Medicine, London, UK (M Hossain MSc, Prof M McKee DSc, Prof N Pearce PhD, R L Pullan PhD, T Tillmann MBChB); Baylor College of Medicine, Houston, TX, USA (P J Hotez PhD); Public Health Division, Secretariat of the Pacific Community, Noumea, New Caledonia (D G Hoy PhD); National Institute of Public Health, Tunis, Tunisia (Prof M Hsairi MD); School of Public Health, Central South University, Changsha, China (G Hu PhD); George Washington University, Washington, DC, USA (Prof C Huang PhD); Qatar University, Doha, Qatar (A Husseini PhD); Aarhus University, Aarhus, Denmark (K M Iburg PhD); National Institute for Health Development, Tallinn, Estonia (K Innos PhD); Graduate School of Medicine (M Inoue PhD), School of Public Health (Prof N Kawakami MD), The University of Tokyo, Tokyo, Japan (K Shibuya MD); American Cancer Society, New York, NY, USA (F Islami PhD); George Mason University, Fairfax, VA, USA (K H Jacobsen PhD); Graduate School of Public Health, Yonsei University, Seoul, South Korea (Prof S Jee PhD); Centre for Chronic Disease Control, New Delhi, India (P Jeemon PhD, D Prabhakaran DM); Tianjin Centers for Disease Control and Prevention, Tianjin, China (G Jiang MD); Department of Ophthalmology, Medical Faculty Mannheim, Ruprecht-KarlsUniversity Heidelberg, Mannheim, Indonesia (Prof J B Jonas MD); The National Institute of Public Health, Copenhagen, Denmark (Prof K Juel PhD); Zhongshan Hospital (J She MD), Fudan University, Shanghai, China (H Kan MD); Department of Epidemiology, Helmholtz Centre for Infection Research, Braunschweig, Germany (A Karch MD); HannoverBraunschweig Site, German Center for Infection Research (DZIF), Braunschweig, Germany (A Karch MD); Malaria and Other Parasitic Diseases Division, Ministry of Health, Kigali City, Rwanda (C K Karema MSc); Case Western University Hospitals, Cleveland, OH, USA (C Karimkhani MD); All India Institute of Medical Sciences, New Delhi, India (Prof G Karthikeyan DM, V K Paul MD, Prof N Tandon PhD, M Satpathy PhD); Oklahoma State University, Tulsa, OK, USA (A Kaul MD); National Center for Disease Control and Public Health, Tbilisi, Georgia (K Kazanjan MS); South African Medical Research Council, Cape Town, South Africa (A P Kengne PhD, R Matzopoulos PhD, W T Msemburi MPhil, Prof D J Stein MD); Faculty of Health Sciences, Hatter Institute for Cardiovascular Research in Africa (Prof K Sliwa PhD), School of Public Health and Family Medicine (R Matzopoulos $\mathrm{PhD}$ ), University of Cape Town, Cape Town, South Africa (A P Kengne PhD, Prof B M 
Mayosi DPhil, Prof D J Stein MD); Cardiology, Hadassah Ein Kerem University Hospital, Jerusalem, Israel (Prof A Keren MD); Jordan University of Science and Technology, Irbid, Jordan (Prof Y S Khader ScD); Supreme Council of Health, Doha, Qatar (S A Khalifa MSc); Health Services Academy, Islamabad, Pakistan (E A Khan MPH); Expanded Programme on Immunization, Islamabad, Pakistan (E A Khan MPH); UAE University, Al Ain, United Arab Emirates (G Khan PhD); Seoul National University College of Medicine, Seoul, South Korea (Prof Y Khang MD); Federal University of Rio Grande do Sul, Porto Alegre, Brazil (C Kieling PhD); Northeastern University, Boston, MA, USA (Prof D Kim DrPH); Soonchunhyang University, Seoul, South Korea (S Kim PhD); Southern University College, Johor, Malaysia (Y Kim PhD); University of Canberra, Canberra, ACT, Australia (Y Kinfu PhD); Department of Health Registries (A Knudsen PhD), Norwegian Institute of Public Health, Oslo, Norway (J M Kinge PhD, Prof V Skirbekk MD, Prof S Vollset MD); National Cerebral and Cardiovascular Center, Suita, Japan (Y Kokubo PhD); Center for Community Empowerment, Health Policy and Humanities, NIHRD, Jakarta, Indonesia (S Kosen MD); Oregon Health and Science University, Portland, OR, USA (S Krishnaswami MD, D Zonies MD); University of Montreal, Montreal, QC, Canada (Prof B Kuate Defo $\mathrm{PhD}$ ); Erasmus MC, University Medical Center Rotterdam, Rotterdam, Netherlands (Prof E J Kuipers PhD, S Polinder MS, Prof J Richardus PhD); Rajrajeswari Medical College \& Hospital, Bangalore, India (Prof C Kulkarni MD); Arkansas State University, Jonesboro, AR, USA (V S Kulkarni PhD); Fourth View Consulting, Tallinn, Estonia (T Lai PhD); Australian Research Centre for Population Oral Health, School of Dentistry, The University of Adelaide, Adelaide, SA, Australia (Prof R Lalloo PhD); Disability Prevention Research Centre (T Lallukka PhD), Finnish Institute of Occupational Health, Helsinki, Finland (R Shiri PhD); Faculty of Medicine, University of Helsinki, Helsinki, Finland (T Lallukka $\mathrm{PhD}$ ); Institute of Health Policy and Development Studies, National Institutes of Health, Manila, Philippines (Prof H Lam PhD); National Cancer Institute, Rockville, MD, USA (Q Lan PhD); Help Me See, Inc, New York, NY, USA (V C Lansingh PhD); Instituto Mexicano de Oftalmologia, Queretaro, Mexico (V C Lansingh PhD); Uppsala University, Uppsala, Sweden (Prof A Larsson PhD); Instituto Nacional de Epidemiología "Dr. Juan H. Jara", Mar del Plata, Argentina (A E B Lawrynowicz MPH); Nova Southeastern University College of Optometry, Fort Lauderdale, FL, USA (J L Leasher OD); SUNY-Albany, Rensselaer, NY, USA (R Leung PhD); Jinan Central Hospital, Jinan, China (B Li PhD); National Center for Chronic and Noncommunible Disease Control and Prevention, China CDC, Beijing, China (Y Li MPH, S Liu PhD, L Duan MS, Prof L Wang MD, P Ye MPH); Anolinx, LLC, Salt Lake City, UT, USA (Y Li PhD); Wayne State University, Miami, FL, USA (Prof S E Lipshultz MD); Rollins School of Public Health (E P Simard PhD), Emory University, Atlanta, GA, USA (Prof Y Liu PhD, Prof K Narayan MD); Turning Point, Eastern Health, Melbourne, VIC, Australia (B K Lloyd PhD); University of Bari, Bari, Italy (Prof G Logroscino PhD); University of Bristol, Bristol, UK (K J Looker PhD); American Cancer Society, Atlanta, GA, USA (J Lortet-Tieulent MSc); The Australian National University, Canberra, ACT, Australia (Prof R M Lucas PhD); Aintree University Hospitals NHS Foundation Trust, Liverpool, UK (Prof R Lunevicius PhD); Swansea University, Swansea, UK (Prof R A Lyons MD); Ministry of Health Singapore, Singapore, Singapore (S Ma $\mathrm{PhD}$ ); Saw Swee Hock School of Public Health, National University of Singapore, Singapore, Singapore (S Ma PhD); Murdoch Children's Research Institute (R G Weintraub 
MBBS), Royal Children's Hospital, Melbourne, VIC, Australia (M T Mackay MBBS); Digestive Disease Reseach Institute, Shariati Hospital, Tehran, Iran (Prof R Malekzadeh MD); Shiraz University of Medical Scinces, Shiraz, Iran (Prof R Malekzadeh MD); Queen Mary University of London, London, UK (Prof W Marcenes PhD); Pereleman School of Medicine (P A Meaney MD), University of Pennsylvania, Philadelphia, PA, USA (D J Margolis PhD, D H Silberberg MD); University of the East Ramon Magsaysay Memorial Medical Center, Quezon City, Philippines (M B Marzan MSc); Elmhurst Hospital Center, Mount Sinai Services, Elmhurst, NY, USA (J R Masci MD); Ministry of Public Health, Kabul, Afghanistan (M T Mashal PhD); AIDC EC, Port Elizabeth, South Africa (T T Mazorodze MA); Royal Prince Alfred Hospital, Camperdown, NSW, Australia (N W McGill MB BS); Children's Hospital of Philadelphia, Philadelphia, PA, USA (P A Meaney MD); Janakpuri Superspecialty Hospital, New Delhi, India (Prof M Mehndiratta DM); Mekelle University, Mekelle, Ethiopia (Y A Melaku MPH); Thomas Jefferson University, Philadelphia, PA, USA (M Meltzer MD); Saudi Ministry of Health, Riyadh, Saudi Arabia (Prof Z A Memish MD); Department of Neurology, Helsinki University Central Hospital, Helsinki, Finland (A Meretoja PhD); Ifakara Health Institute, Bagamoyo, Tanzania (F A Mhimbira MS); Pacific Institute for Research \& Evaluation, Calverton, MD, USA (T R Miller PhD); Curtin University Centre for Population Health, Perth, WA, Australia (T R Miller PhD); University of Ottawa, Ottawa, ON, Canada (E J Mills PhD); Department of Medicine, Universiti Kebangsaan Malaysia Medical Center, Bandar Tun Razak, Malaysia (Prof N Mohamed Ibrahim MRCP); University of Salahaddin, Erbil, Iraq (K A Mohammad $\mathrm{PhD}$ ); University of Papua New Guinea, Boroko, Papua New Guinea (Prof G L D Mola MD); Institute for Maternal and Child Health, IRCCS "Burlo Garofolo", Trieste, Italy (L Monasta DSc, L Ronfani PhD, M Montico Msc); University of North Texas, Denton, TX, USA (Prof A R Moore PhD); Department of Community Medicine, Iran University of Medical Sciences, Tehran, Iran (M Moradi-Lakeh PhD); National Center for Child Health and Development, Setagaya, Japan (R Mori PhD); Department of Medicine (Prof M Tsilimbaris PhD), University of Crete, Crete, Greece (J Moschandreas PhD); Egerton University, Egerton, Kenya (W N Moturi PhD); Philipps-University Marburg, Marburg, Germany (Prof U O Mueller PhD); Okinawa Chubu Hospital, Okinawa, Japan (M Mukaigawara MD); West Herts Hospitals NHS Trust, Watford, UK (M E Murdoch FRCP); University of KwaZulu-Natal, Durban, South Africa (Prof K S Naidoo PhD); Azienda Ospedaliera papa Giovanni XXIII, Bergamo, Italy (Prof L Naldi MD); Ministry of Health, Suva, Fiji (D Nand MPH [Hons]); Suraj Eye Institute, Nagpur, India (Prof V Nangia MD); Faculty of Medicine, Fez, Morocco (Prof C Nejjari PhD); University of Oslo, Oslo, Norway (S P Neupane PhD); Kilifi, Kenya (Prof C R Newton MD); Ministry of Health and Social Welfare, Dar es Salaam, Tanzania (F N Ngalesoni MSc); Charité University Medicine Berlin, Berlin, Germany (S Nolte PhD, C Papachristou PhD); Deakin University, Melbourne, VIC, Australia (S Nolte PhD); Department of Global Public Health and Primary Care (Prof S Vollset MD), University of Bergen, Bergen, Norway (Prof O F Norheim PhD); Institute of Health and Biomedical Innovation, Queensland University of Technology, Brisbane, QLD, Australia (R E Norman PhD); Department of Clinical Sciences, Lund University, Lund, Sweden (Prof B Norrving PhD); Makerere University, Kampala, Uganda (L Nyakarahuka MPH); Kyung Hee University, Seoul, South Korea (Prof I Oh PhD); Teikyo University School of Medicine, Tokyo, Japan (Prof T Ohkubo MD); Center for Healthy Start Initiative, 
Ikoyi, Nigeria (B O Olusanya PhD); Lira District Local Government, Lira Municipal Council, Uganda (J N Opio MPH); IIS-Fundacion Jimenez Diaz, Madrid, Spain (Prof A Ortiz PhD); Christian Medical College Ludhiana, Ludhiana, India (J D Pandian DM); Kosin University College of Medicine, Busan, South Korea (E Park PhD); School of Medicine, Sungkyunkwan University, Suwon, South Korea (Prof J Park MPH); Independent Researcher, Boroko, Papua New Guinea (B I Pavlin MD); REQUIMTE/LAQV, Laboratório de Farmacognosia, Departamento de Química, Faculdade de Farmácia, Universidade do Porto, Porto, Portugal (Prof D M Pereira PhD); National Institute of Respiratory Diseases, Mexico City, Mexico (Prof R Perez-Padilla MD); Hopsital Universitario Cruces, OSI EECruces, Barakaldo, Spain (F Perez-Ruiz PhD); Biocruces Health Research Institute, Baracaldo, Spain (F Perez-Ruiz PhD); Mario Negri Institute for Pharmacological Research, Ranica, Italy (N Perico MD, M Trillini MD); Postgraduate Medical Institute, Lahore, Pakistan (A Pervaiz MHA); Flinders University, Adelaide, SA, Australia (Prof K Pesudovs $\mathrm{PhD}$ ); Aalborg University, Aalborg Esst, Denmark (C B Peterson PhD); Health Metrics Unit, Gothenburg, Sweden (Prof M Petzold PhD); The University of the Witwatersrand, Johannesburg, South Africa (Prof M Petzold PhD); Shanghai Jiao Tong University School of Medicine, Shanghai, China (Prof M R Phillips MD); Emory University, Atlanta, GA, USA (Prof M R Phillips MD); Section Exposure Assessment and Environmental Health Indicators, Federal Environment Agency, Berlin, Germany (D Plass Dr. PH); Montreal Children's Hospital, McGill University, Montreal, QC, Canada (Prof D Poenaru MD); MyungSung Medical College, Addis Ababa, Ethiopia (Prof D Poenaru MD); Centre for Addiction and Mental Health (CAMH), Toronto, ON, Canada (S Popova PhD); The Fred Hollows Foundation, Sydney, NSW, Australia (N M Prasad DO); University of Illinois, Chicago, IL, USA (D M Qato PhD); Contech International Health Consultants, Lahore, Pakistan (A Rafay MS); Hamad Medical Corporation, Doha, Qatar (S U Rahman FCPS); University of Missouri, Columbia, MO, USA (M Raju PhD); Contech School of Public Health, Lahore, Pakistan (A Rafay MS, Prof S M Rana PhD); Contech Internal Health Consultants, Lahore, Pakistan (Prof S M Rana PhD); Center for Disease Analysis, Louisville, CO, USA (H Razavi PhD); Suez Canal University, Ismailia, Egypt (Prof A Refaat $\mathrm{PhD}$ ); Centro Anna Maria Astori, IRCCS Mario Negri Institute for Pharmacological Research, Bergamo, Italy (Prof G Remuzzi MD); International Health and Development, Geneva, Switzerland (Prof S ResnikoffMD); Hospital das Clinica da Universidade Federal de Minas Gerais, Belo Horizonte, Brazil (Prof A L Ribeiro PhD); Centre for Research in Environmental Epidemiology (CREAL), Barcelona, Spain (D Rojas-Rueda PhD); Institute of Epidemiology and Medical Biometry, Ulm University, Ulm, Germany (Prof D Rothenbacher MD); Ann and Robert H Lurie Children's Hospital of Chicago, Chicago, IL, USA (D H Rothstein MD); Independent researcher, London, UK (J T Rowley PhD); BARC Hospital, HBNI University, Mumbai, India (N Roy MD); Muhimbili University of Health and Allied Sciences, Dar es Salaam, Tanzania (G M Ruhago MA, B F Sunguya MSc); Queensland Centre for Mental Health Research, The Park Centre for Mental Health, Brisbane, QLD, Australia (S Saha PhD); Case Western Reserve University, Cleveland, OH, USA (J R Sanabria MD); Cancer Treatment Centers of America-RFU Chicago Medical School, North Chicago, IL, USA (J R Sanabria MD); Marshall University, Huntington, WV, USA (M Sawhney PhD); Federal University of Santa Catarina, Florianópolis, Brazil (I J Schneider PhD); University of Alabama at Birmingham, Birmingham, AL, USA (D C 
Schwebel PhD, J A Singh MD); Stellenbosch University, Cape Town, South Africa (Prof S Seedat PhD); An-Najah University, Nablus, Palestine (A Shaheen PhD); Tufts Medical Center, Boston, MA, USA (Prof S Shahraz PhD); University of Pittsburgh Medical Center, Pittsburgh, PA, USA (S Shangguan); Tachikawa Hospital, Tokyo, Japan (Y Shinohara PhD); Finnish Institute of Occupational Health, Helsinki, Finland (R Shiri PhD); Washington State University, Spokane, WA, USA (K Shishani PhD); Northumbria University, Newcastle upon Tyne, UK (I Shiue PhD); Alzheimer's Scotland Dementia Research Centre, University of Edinburgh, Edinburgh, UK (I Shiue PhD); Reykjavik University, Reykjavik, Iceland (I D Sigfusdottir PhD); International Institute for Population Sciences, Mumbai, India (A Singh $\mathrm{PhD}$ ); Dartmouth College, Lebanon, NH, USA (S Soneji PhD); Stavanger University Hospital, Stavanger, Norway (K Søreide PhD); Federal Research Institute for Health Organization and Informatics, Ministry of Health of the Russian Federation, Moscow, Russia (S Soshnikov PhD); Department of Clinical Neurological Sciences, Western University, London, ON, Canada (L A Sposato MD); Faculty of Medicine and Health Sciences, Universiti Tunku Abdul Rahman, Kajang, Cheras, Malaysia (C T Sreeramareddy MPH); “Attikon” University Hospital, Athens, Greece (V Stathopoulou PhD); Department of Neuroscience (Prof L J Stovner PhD), Norwegian University of Science and Technology, Trondheim, Norway (Prof T J Steiner PhD); Norwegian Advisory Unit on Headache, St Olavs Hospital, Trondheim, Norway (Prof L J Stovner PhD); “Alexandra” General Hospital of Athens, Athens, Greece (K Stroumpoulis PhD); Centre Hospitalier Public du Cotentin, Cherbourg, France (K Stroumpoulis); National Institute for Research in Tuberculosis, Chennai, India (S Swaminathan MD); Northwestern University, Chicago, IL, USA (M Swaroop MD, Y Yano MD); University of California Irvine, Irvine, CA, USA (Prof B L Sykes PhD); University of Illinois at Urbana-Champaign, Champaign, IL, USA (K M Tabb $\mathrm{PhD}$ ); Chaim Sheba Medical Center, Tel Hashomer, Israel (Prof D Tanne MD); Tel Aviv University, Tel Aviv, Israel (Prof D Tanne MD); Westchester Medical Center, Valhalla, NY, USA (M Tavakkoli MD); Netherlands Institute of Mental Health and Addiction, Utrecht, Netherlands (M Ten Have PhD); Memorial University, St John's, NL, Canada (E Y Tenkorang PhD); Department of Anesthesiology, University of Virginia, Charlottesville, VA, USA (A S Terkawi MD); Outcomes Research Consortium, Cleveland, OH, USA (A S Terkawi); Department of Anesthesiology, King Fahad Medical City, Riyadh, Saudi Arabia (A S Terkawi); WorldFish, Penang, Malaysia (A L Thorne-Lyman ScD); Aristotle University of Thessaloniki, Thessaloniki, Greece (Prof F Topouzis PhD); Health Care Center of Anjo Kosei Hospital, Anjo City, Japan (H Toyoshima MD); University of Southern Santa Catarina, Palhoça, Brazil (Prof J Traebert PhD); Johns Hopkins University, Baltimore, MD, USA (B X Tran PhD); Hanoi Medical University, Hanoi, Vietnam (B X Tran PhD); Department of Neurology, Rigshospitalet, University of Copenhagen, Copenhagen, Denmark (T Truelsen DSc); Cleveland Clinic, Cleveland, OH, USA (Prof E M Tuzcu MD); Department of Veterans Affairs, Washington, DC, USA (U S Uchendu MD); Department of Internal Medicine Federal Teaching Hospital, Abakaliki, Nigeria (K N Ukwaja MD); Netherlands Leprosy Relief, Amsterdam, Netherlands (W H van Brakel PhD); African Population and Health Research Center, Nariobi, Kenya (S van de Vijver MD); National Institute for Public Health and the Environment, Bilthoven, Netherlands (C $\mathrm{H}$ van Gool $\mathrm{PhD}$ ); Maastricht University Medical Centre, Maastricht, Netherlands (Prof J van Os PhD); UKK Institute for Health Promotion Research, Tampere, Finland (Prof T J Vasankari PhD); 
Neuroscience Centre, Raffles Hospital, Singapore, Singapore (N Venketasubramanian MD); University of Bologna, Bologna, Italy (Prof F S Violante MD); Higher School of Economics, Moscow, Russia (Prof V V Vlassov MD); National Institute for Occupational Safety and Health, Washington, DC, USA (G R Wagner MD); Uniformed Services University of Health Sciences, Bethesda, MD, USA (Prof S G Waller MD); Institute of Basic Medical Sciences (X Wan PhD), and Cancer Institute (W Chen PhD, Prof X Zou MD), Chinese Academy of Medical Sciences, Beijing, China; NIHRD, Ministry of Health Indonesia, Jakarta, Indonesia (T S Warouw PhD); Health Canada, Ottawa, ON Canada (S Weichenthal PhD); Beijing Neurosurgical Institute, Beijing, China (Prof W Wenzhi MD); Institute of Medical Sociology and Social Medicine, Marburg, Germany (A Werdecker DiplOecTroph.); Federal Institute for Population Research, Wiesbaden, Germany (R Westerman PhD); German National Cohort Consortium, Heidelberg, Germany (R Westerman PhD); Wayne State University, Detroit, MI, USA (Prof J D Wilkinson MD); Royal Cornwall Hospital, Truro, UK (Prof A D Woolf FRCP); Nanjing University School of Medicine, Nanjing, China (Prof G Xu PhD); Duke Kunshan University, Kunshan, China (Prof L L Yan PhD); Jichi Medical School, Tochigi, Japan (Y Yano MD); The University of Hong Kong, Hong Kong Special Administrative Region, China (Prof P Yip PhD); National Center of Neurology and Psychiatry, Kodaira, Japan (N Yonemoto MPH); Korea University, Seoul, South Korea (S Yoon PhD); Jackson State University, Jackson, MS, USA (Prof M Z Younis PhD); School of Public Health, Wuhan, China (Prof C Yu PhD); Global Health Institute, Wuhan University, Wuhan, China (Prof C Yu PhD); Mansoura Faculty of Medicine, Mansoura, Egypt (Prof M E Zaki MD); and Chongqing Medical University, Chongqing, China (Prof Y Zhao MSc)

\section{Declaration of interests}

BDG works for AMP, which receives grant-specific support from Crucell, GlaxoSmithKline, Merck, Novartis, Pfizer, and Sanofi Pasteur; however, none of this support is for work related to the present report. MGS received a speaking honorarium from Ethicon for work unrelated to this manuscript. MBS is a paid consultant to Janssen, Pfizer, and Tonix Pharmaceuticals and is also paid for his editorial work on $U p$-to-Date and on the journal Biological Psychiatry. PJ is supported by a career development fellowship from the Wellcome Trust, Public Health Foundation of India and a Consortium of UK Universities. SIH is funded by a Wellcome Trust Grant. JAS has received grant support from Takeda and Savient Pharmaceuticals and consultant fees from Takeda, Regeneron, Allergan, and Savient. JAS is an executive member of OMERACT, an organisation that received armslength funding from 36 pharmaceutical companies. KJL was funded by WHO to conduct the review of HSV-2 seroprevalence which informs this study. During the study, KJL received funding from Health Protection Scotland, the National Institute for Health Research (NIHR) Health Protection Research Unit (HPRU) in Evaluation of Interventions, and Sexual Health 24: the funding sources had no role in the writing of the manuscript or the decision to submit it for publication. FP-R is a consultant for AstraZeneca, Cimabay, Menarini, and Pfizer, and has received investigation grants from the Spanish Health Ministry, Spanish Foundation for Rheumatology, and Cruces Hospital Rheumatology Association. PJH is principal investigator on vaccines in clinical trials against hookworm and schistosomiasis as well as 
several other neglected tropical disease vaccines in development. CKi receives research grants from Brazilian public funding agencies Conselho Nacional de Desenvolvimento Científico e Tecnológico (CNPq), Coordenação de Aperfeiçoamento de Pessoal de Nível Superior (CAPES), and Fundação de Amparo à Pesquisa do Estado do Rio Grande do Sul (FAPERGS). CKi has also received authorship royalties from publishers Artmed and Manole. KBG received the NHMRC-Gustav Nossal scholarship sponsored by CSL in 2012. This award is peer-reviewed through the standard NHMRC peer-review process; CSL played no part in selection of the awardee. HAW, AJF, FJC, and HEE are all affiliated with the Queensland Centre for Mental Health Research, which receives funding from the Queensland Department of Health. DJS has received research grants and consultancy honoraria in the past three years from AMBRF, Biocodex, Cipla, Lundbeck, National Responsible Gambling Foundation, Novartis, Servier, and Sun. DJS is also supported by the Medical Research Council of South Africa. DM reports being on the scientific advisory board of Unilever North America and ad hoc honoraria or consulting from Bunge, Nutrition Impact, Amarin, AstraZeneca, and Life Sciences Research Organization. DAQ was supported by The Eunice Kennedy Shriver National Institute of Child Health and Human Development of the National Institutes of Health under award number 5T32HD057822. RAL receives funding through the Farr Institute of Health Informatics Research. The Farr Institute is supported by a consortium of ten UK research organisations: Arthritis Research UK, the British Heart Foundation, Cancer Research UK, the Economic and Social Research Council, the Engineering and Physical Sciences Research Council, the Medical Research Council, the National Institute of Health Research, the National Institute for Social Care and Health Research (Welsh Government), and the Chief Scientist Office (Scottish Government Health Directorates). KeS is affiliated with Grant in Aid for Scientific Research from the Ministry of Education, Culture, Sports and Technology in Japan. JM received a fellowship from the Wellcome Trust. AKau acknowledges that she receives funding from Oklahoma Center for the Advancement of Science and Technology (OCAST) as co-principal investigator. HoC acknowledges that the study was in part supported by the intramural research program of the NIH, the National Institute of Environmental Health Sciences. SS received research support and funding from the NIH and NRF and an honorarium from Pharmaceutical companies and is an employee at the NRF. VC acknowledges the following conflicts of interest: Speaker Bureau Boehringer Ingelheim, BMS Pfizer Advisory board: Boehringer Ingelheim Mindmaze. All other authors declare no competing interests.

\section{References}

1. Global Burden of Disease Study 2013 Collaborators. Global, regional, and national incidence, prevalence, and years lived with disability for 301 acute and chronic diseases and injuries in 188 countries, 1990-2013: a systematic analysis for the Global Burden of Disease Study 2013. Lancet. 2015; 386:743-800. [PubMed: 26063472]

2. GBD 2013 Mortality and Causes of Death Collaborators. Global, regional, and national age-sex specific all-cause and cause-specific mortality for 240 causes of death, 1990-2013: a systematic analysis for the Global Burden of Disease Study 2013. Lancet. 2014; 385:117-71. [PubMed: 25530442]

3. Alliance NCD. Healthy planet, healthy people: The NCD alliance vision for health in the post-2015 development agenda. NCD Alliance; Geneva: 2013. 
4. [accessed Feb 9, 2015] Open working group proposal for Sustainable development goals. 2014. enthttps://sustainabledevelopment.un.org/index.php?page=view \&type $=400 \& n r=1579 \&$ menu $=1300$

5. Pintér, L.; Almássy, D.; Hatakeyama, S. Sustainable Development Goals and indicators for a small planet—Part II: Measuring sustainability. Asia-Europe Foundation; Singapore: 2014.

6. Murray CJ, Salomon JA, Mathers C. A critical examination of summary measures of population health. Bull World Health Organ. 2000; 78:981-94. [PubMed: 10994282]

7. Murray CJL, Vos T, Lozano R, et al. Disability-adjusted life years (DALYs) for 291 diseases and injuries in 21 regions, 1990-2010: a systematic analysis for the Global Burden of Disease Study 2010. Lancet. 2012; 380:2197-223. [PubMed: 23245608]

8. Salomon JA, Wang H, Freeman MK, et al. Healthy life expectancy for 187 countries, 1990-2010: a systematic analysis for the Global Burden Disease Study 2010. Lancet. 2012; 380:2144-62. [PubMed: 23245606]

9. Santosa A, Wall S, Fottrell E, Högberg U, Byass P. The development and experience of epidemiological transition theory over four decades: a systematic review. Glob Health Action. 2014; 7:23574. [PubMed: 24848657]

10. Murray CJL, Ortblad KF, Guinovart C, et al. Global, regional, and national incidence and mortality for HIV, tuberculosis, and malaria during 1990-2013: a systematic analysis for the Global Burden of Disease Study 2013. Lancet. 2014; 384:1005-70. [PubMed: 25059949]

11. Hill K, Vapattanawong P, Prasartkul P, Porapakkham Y, Lim SS, Lopez AD. Epidemiologic transition interrupted: a reassessment of mortality trends in Thailand, 1980-2000. Int J Epidemiol. 2007; 36:374-84. [PubMed: 17182635]

12. Hofmarcher MM. Is public health between East and West? Analysis of wealth, health and mortality in Austria, Central and Eastern European Countries and Croatia relative to the European Union. Croat Med J. 1998; 39:241-48. [PubMed: 9740635]

13. Kahn K, Garenne ML, Collinson MA, Tollman SM. Mortality trends in a new South Africa: hard to make a fresh start. Scand J Public Health Suppl. 2007; 69:26-34. [PubMed: 17676500]

14. Rivera JA, Barquera S, Campirano F, Campos I, Safdie M, Tovar V. Epidemiological and nutritional transition in Mexico: rapid increase of non-communicable chronic diseases and obesity. Public Health Nutr. 2002; 5:113-22. [PubMed: 12027273]

15. United Nations General Assembly. Prevention and control of non-communicable diseases: Report of the Secretary General. United Nations; New York, NY: 2011.

16. World Health Organization (WHO). 2008-2013 Action Plan for the Global Strategy for the Prevention and Control of Noncommunicable Diseases: Prevent and control cardiovascular diseases, cancers, chronic respiratory diseases and diabetes. World Health Organization; Geneva, Switzerland: 2009.

17. Yang G, Kong L, Zhao W, et al. Emergence of chronic non-communicable diseases in China. Lancet. 2008; 372:1697-705. [PubMed: 18930526]

18. Yang G, Wang Y, Zeng Y, et al. Rapid health transition in China, 1990-2010: findings from the Global Burden of Disease Study 2010. Lancet. 2013; 381:1987-2015. [PubMed: 23746901]

19. Murray CJL, Ezzati M, Flaxman AD, et al. GBD 2010: design, definitions, and metrics. Lancet. 2012; 380:2063-66. [PubMed: 23245602]

20. Flaxman, A. Integrated Metaregression Framework for Descriptive Epidemiology. University of Washington Press; Seattle, WA: 2014.

21. Salomon JA, Vos T, Hogan DR, et al. Common values in assessing health outcomes from disease and injury: disability weights measurement study for the Global Burden of Disease Study 2010. Lancet. 2012; 380:2129-43. [PubMed: 23245605]

22. Sullivan DF. A single index of mortality and morbidity. HSMHA Health Rep. 1971; 86:347-54. [PubMed: 5554262]

23. Bloom DE, Canning D. Policy forum: public health. The health and wealth of nations. Science. 2000; 287:1207-09. 1209. [PubMed: 10712155]

24. Preston SH. The changing relation between mortality and level of economic development. Popul Stud (Camb). 1975; 29:231-48. [PubMed: 11630494]

25. Jolliffe, I. Encyclopedia of Statistics in Behavioral Science. John Wiley \& Sons; West Sussex: 2005. Principal component analysis. 
26. Gelman A. Analysis of variance-why it is more important than ever. Ann Stat. 2005; 33:1-53.

27. Omran AR. The epidemiologic transition. A theory of the epidemiology of population change. Milbank Mem Fund Q. 1971; 49:509-38. [PubMed: 5155251]

28. Agyei-Mensah S, de-Graft Aikins A. Epidemiological transition and the double burden of disease in Accra, Ghana. J Urban Health. 2010; 87:879-97. [PubMed: 20803094]

29. Frenk J, Bobadilla JL, Stern C, Frejka T, Lozano R. Elements for a theory of the health transition. Health Transit Rev. 1991; 1:21-38. [PubMed: 10148802]

30. Andreev EM, Nolte E, Shkolnikov VM, Varavikova E, McKee M. The evolving pattern of avoidable mortality in Russia. Int J Epidemil. 2003; 32:437-46.

31. McMichael AJ, McKee M, Shkolnikov V, Valkonen T. Mortality trends and setbacks: global convergence or divergence? Lancet. 2004; 363:1155-59. [PubMed: 15064037]

32. Smallman-Raynor M, Phillips D. Late stages of epidemiological transition: health status in the developed world. Health Place. 1999; 5:209-22. [PubMed: 10984576]

33. Carolina Martínez S, Gustavo Leal F. Epidemiological transition: model or illusion? A look at the problem of health in Mexico. Soc Sci Med. 2003; 57:539-50. [PubMed: 12791495]

34. Caselli G, Meslé F, Vallin J. Epidemiologic transition theory exceptions. Genus. 2002; 58:9-51.

35. Rivera-Andrade A, Luna MA. Trends and heterogeneity of cardiovascular disease and risk factors across Latin American and Caribbean countries. Prog Cardiovasc Dis. 2014; 57:276-85. [PubMed: 25218566]

36. Bloom DE, Canning D. Commentary: The Preston Curve 30 years on: still sparking fires. Int J Epidemiol. 2007; 36:498-99. discussion 502-03. [PubMed: 17550948]

37. Mackenbach JP, Looman CW. Life expectancy and national income in Europe, 1900-2008: an update of Preston's analysis. Int J Epidemiol. 2013; 42:1100-10. [PubMed: 23920140]

38. Preston SH. The changing relation between mortality and level of economic development. Population Studies, Vol. 29, No. 2, July 1975. 2007; 36:484-90.

39. Bergström G, Redfors B, Angerås O, et al. Low socioeconomic status of a patient's residential area is associated with worse prognosis after acute myocardial infarction in Sweden. Int J Cardiol. 2015; 182:141-47. [PubMed: 25577750]

40. Capingana DP, Magalhães P, Silva ABT, et al. Prevalence of cardiovascular risk factors and socioeconomic level among public-sector workers in Angola. BMC Public Health. 2013; 13:732. [PubMed: 23924306]

41. Chang P, Nead KT, Olin JW, Cooke JP, Leeper NJ. Clinical and socioeconomic factors associated with unrecognized peripheral artery disease. Vasc Med. 2014; 19:289-96. [PubMed: 24872403]

42. Di Cesare M, Khang Y-H, Asaria P, et al. the Lancet NCD Action Group. Inequalities in noncommunicable diseases and effective responses. Lancet. 2013; 381:585-97. [PubMed: 23410608]

43. Marmot MG, Kogevinas M, Elston MA. Social/economic status and disease. Annu Rev Public Health. 1987; 8:111-35. [PubMed: 3555518]

44. Marmot MG, Shipley MJ, Rose G. Inequalities in death-specific explanations of a general pattern? Lancet. 1984; 1:1003-06. [PubMed: 6143919]

45. Marmot MG, Smith GD, Stansfeld S, et al. Health inequalities among British civil servants: the Whitehall II study. Lancet. 1991; 337:1387-93. [PubMed: 1674771]

46. Wang J-Y, Wang C-Y, Juang S-Y, et al. Low socioeconomic status increases short-term mortality of acute myocardial infarction despite universal health coverage. Int J Cardiol. 2014; 172:82-87. [PubMed: 24444479]

47. Wu W-H, Yang L, Peng F-H, et al. Lower socioeconomic status is associated with worse outcomes in pulmonary arterial hypertension. Am J Respir Crit Care Med. 2013; 187:303-10. [PubMed: 23220911]

48. Robine, J-M.; Mathers, CD.; Bone, MR.; Romieu, I. Calculation of Health Expectancies: Harmonization, Consensus Achieved and Future Perspectives. John Libbey Eurotext; Paris: 1993.

49. Jagger C, Gillies C, Moscone F, et al. the EHLEIS team. Inequalities in healthy life years in the 25 countries of the European Union in 2005: a cross-national meta-regression analysis. Lancet. 2008; 372:2124-31. [PubMed: 19010526] 
50. King G, Murray CJL, Salomon JA, Tandon A. Enhancing the validity and cross-cultural comparability of measurement in survey research. Am Polit Sci Rev. 2004; 98:191-207.

51. Salomon JA, Nordhagen S, Oza S, Murray CJL. Are Americans feeling less healthy? The puzzle of trends in self-rated health. Am J Epidemiol. 2009; 170:343-51. [PubMed: 19564169]

52. Salomon JA, Tandon A, Murray CJL. Comparability of self rated health: cross sectional multicountry survey using anchoring vignettes. BMJ. 2004; 328:258. [PubMed: 14742348]

53. Gennaro V, Ghirga G, Corradi L. In Italy, healthy life expectancy drop dramatically: from 2004 to 2008 there was a 10 years drop among newborn girls. Ital J Pediatr. 2012; 38:19. [PubMed: 22607773]

54. Catalá-López F, García-Altés A, Alvarez-Martín E, Gènova-Maleras R, Morant-Ginestar C. Does the development of new medicinal products in the European Union address global and regional health concerns? Popul Health Metr. 2010; 8:34. [PubMed: 21172012]

55. Dorsey ER, Thompson JP, Carrasco M, et al. Financing of U.S. biomedical research and new drug approvals across therapeutic areas. PLoS One. 2009; 4:e7015. [PubMed: 19750225]

56. Gross CP, Anderson GF, Powe NR. The relation between funding by the National Institutes of Health and the burden of disease. N Engl J Med. 1999; 340:1881-87. [PubMed: 10369852]

57. Keyhani S, Wang S, Hebert P, Carpenter D, Anderson G. US pharmaceutical innovation in an international context. Am J Public Health. 2010; 100:1075-80. [PubMed: 20403883]

58. Luengo-Fernandez R, Leal J, Gray AM. UK research expenditure on dementia, heart disease, stroke and cancer: are levels of spending related to disease burden? Eur J Neurol. 2012; 19:149_ 54. [PubMed: 21951976]

59. Moses H 3rd, Matheson DHM, Cairns-Smith S, George BP, Palisch C, Dorsey ER. The anatomy of medical research: US and international comparisons. JAMA. 2015; 313:174-89. [PubMed: 25585329]

60. Foreman KJ, Lozano R, Lopez AD, Murray CJ. Modeling causes of death: an integrated approach using CODEm. Popul Health Metr. 2012; 10:1. [PubMed: 22226226] 


\section{Research in context}

\section{Evidence before this study}

In 2012, results from the first complete revision of the Global Burden of Disease (GBD) since the first assessment in 1993 became available. This effort was called the GBD 2010 study and reported on disability-adjusted life-years (DALYs) and health-adjusted life expectancy (HALE) by country for 1990 and 2010 based on analyses of an extensive data collection effort to collate all available information on causes of death and disease occurrence in 187 countries. In response to the need for up-to-date information about the health of populations to inform health policy decision making, a decision was made to produce annual updates. The GBD 2013 is the first of these annual updates. In previous papers on the GBD 2013 study, we have documented the new data and new methods used to assess mortality and morbidity by country and over time.

\section{Added value of this study}

Here, we present the results for the aggregation of mortality and morbidity in terms of DALYs and HALE by country and for the time period 1990 to 2013. We examined to what extent the changes in DALYs since 1990 by disease and country can be explained by a composite indicator of sociodemographic status, constructed from income per person, years of schooling after age 15 years, median age of the population and total fertility rate. These GBD 2013 results for the period 1990 to 2013 for DALYs and HALE supersede all previously published GBD findings on DALYs and HALE.

\section{Implications of all the available evidence}

Numbers of DALYs and crude and age-standardised DALY rates for communicable diseases, maternal, neonatal, and nutritional disorders have decreased since 1990. For non-communicable diseases, the number of DALYs have increased, crude rates have remained stable, and age-standardised rates have decreased. Global health is improving but population increase and ageing are keeping the crude rates of DALYs constant, showing that progress in health does not mean fewer demands on health systems. The epidemiological transition, as quantified using our sociodemographic status indicator, accounts for much of the variation between countries and over time for most communicable, maternal, and neonatal causes but not for many non-communicable causes such as cardiovascular disease. The large variation in burden that is not associated with sociodemographic status emphasises the need for ongoing detailed assessments of DALYs and HALE at the country level to inform health policies. 


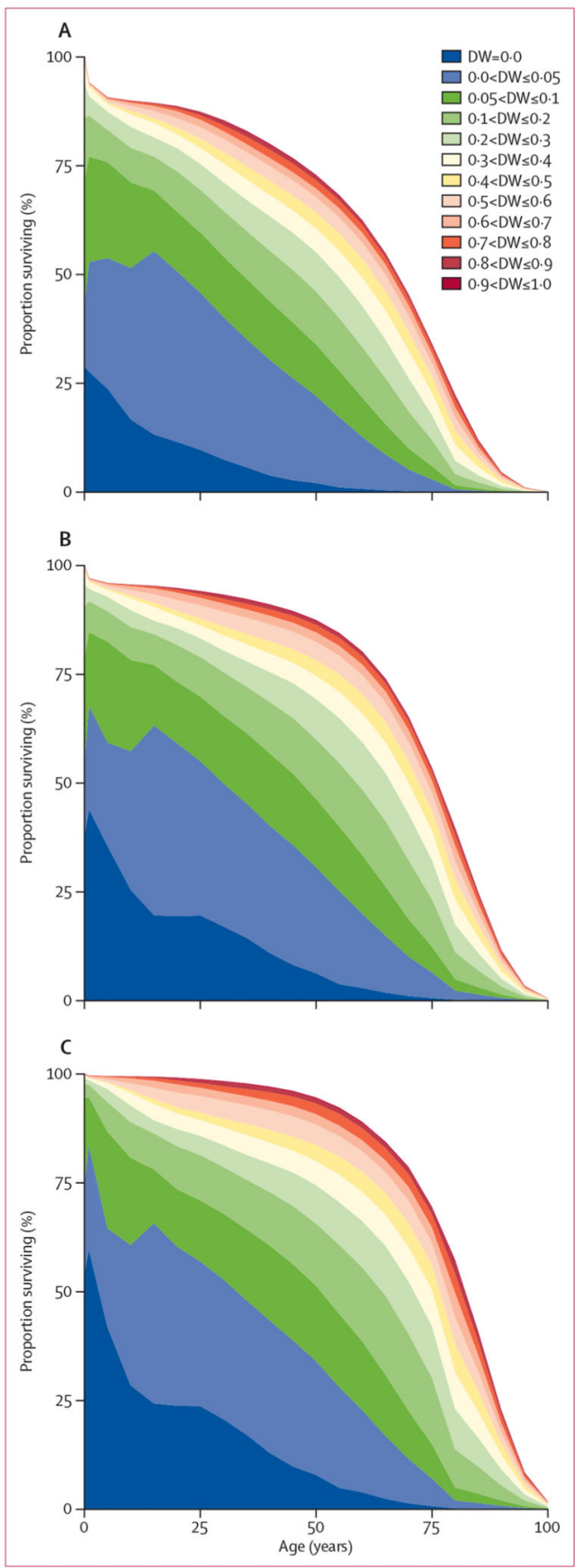

Figure 1. Survivorship curve stratified by disability weight in 2013

Health survivorship function showing the fraction of a birth cohort alive at each age exposed to 2013 death rates, with the fraction of time spent at each age by the birth cohort decomposed by level of disability weight. Countries are grouped by socidemographic status into quintiles, including the lowest quintile (A), the three middle quintiles (B), and the highest quintile (C). DW=disability weight. 


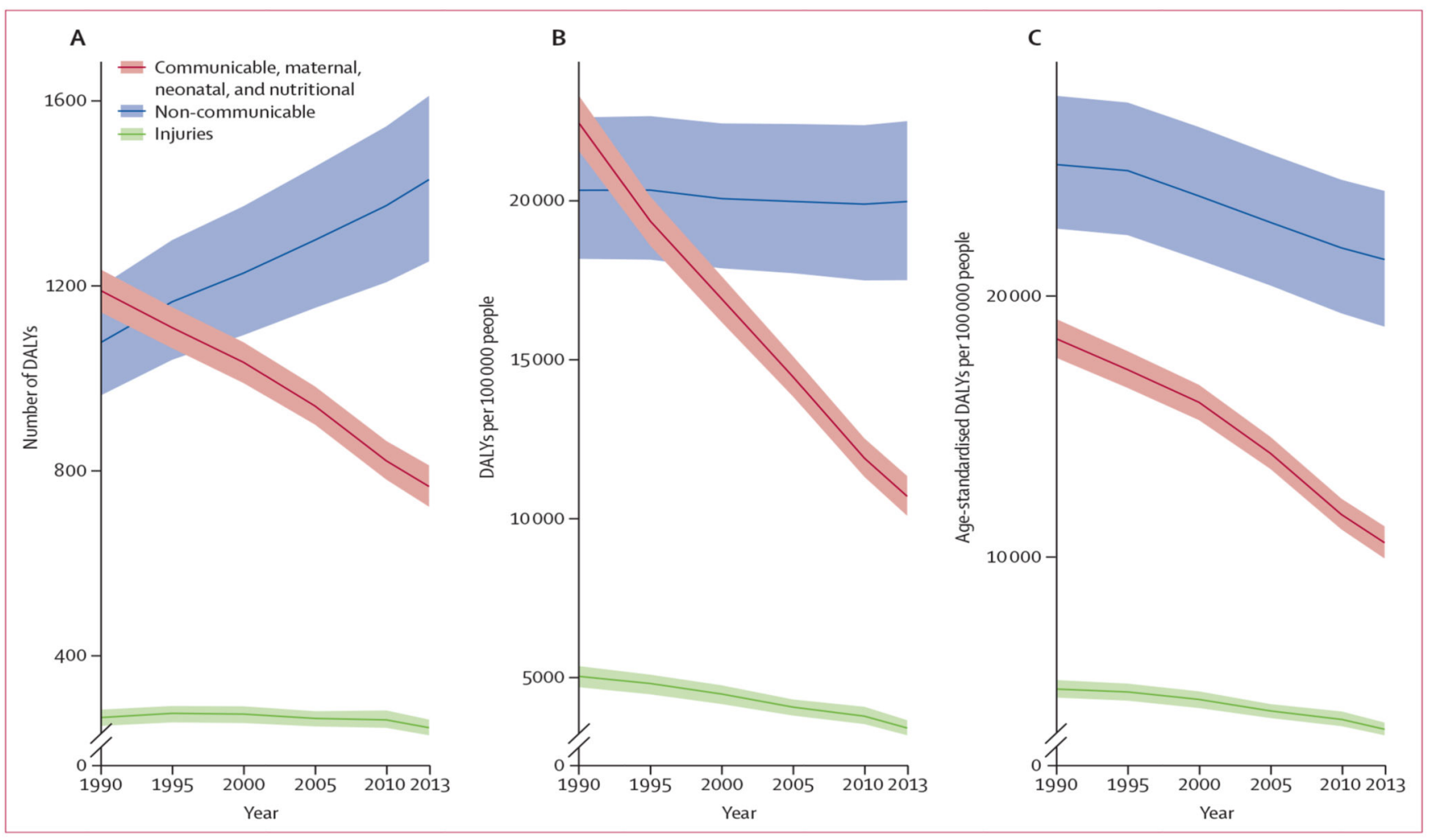

Figure 2. Total DALYs, crude DALY rates, and age-standardised DALY rates from 1990 to 2013 Changes in global DALYs caused by communicable, maternal, neonatal, and nutritional disorders, non-communicable diseases, and injuries shown in terms of numbers of DALYs (A), DALY rates per 100000 people (B), and age-standardised DALY rates per 100000 people (C). The difference in trends between $A$ and $B$ is caused by population growth and the difference between $B$ and $C$ because of changes in the percentage distribution of the population by age. Shaded areas show $95 \%$ uncertainty intervals. DALY=disability-adjusted life-years. 


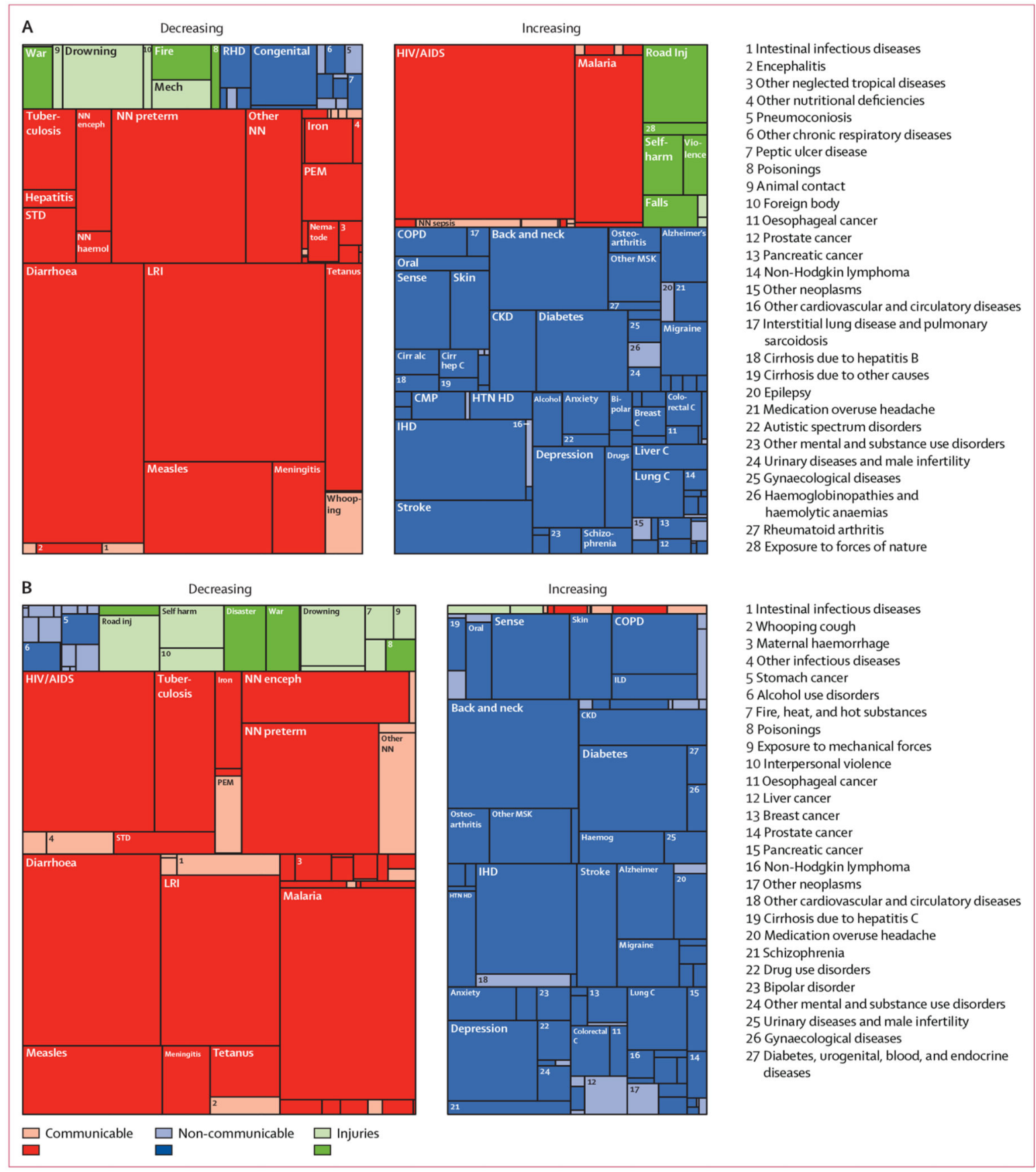

Figure 3. Change in DALYs for GBD level 3 causes

Increasing and decreasing global DALYs for GBD level 3 causes from 1990 to 2005 (A) and 2005 to 2013 (B). Within each tree map, the size of the rectangle for each cause is proportional to the magnitude of the decrease or increase in DALYs for each cause. Dark shading show statistically significant changes and light shading shows changes that are not significant. Unmarked boxes represent causes for which the decrease or increase was less than 1000000 DALYs. Table 1 and the appendix (p 4) contains numerical values for each cause. DALY=disability-adjusted life-years. GBD=Global Burden of Disease. 
Diarrhoea=diarrhoeal diseases. LRI=lower respiratory infections. NN enceph=neonatal encephalopathy due to birth asphyxia and trauma. NN haemol=haemolytic disease and other neonatal jaundice. Other $\mathrm{NN}=$ other neonatal disorders. $\mathrm{NN}$ preterm=preterm birth complications. Nematode=intestinal nematode infections. Iron=iron-deficiency anaemia. PEM=protein-energy malnutrition. STD=sexually transmitted diseases excluding HIV. $\mathrm{TB}=$ tuberculosis. Whooping=whooping cough. $\mathrm{NN}$ sepsis=neonatal sepsis and other neonatal infections. Congenital=congenital anomalies. RHD=rheumatic heart disease. Oral=oral disorders. Sense=sense organ diseases. Cirr alc=cirrhosis due to alcohol use. Cirr hep $\mathrm{C}=$ cirrhosis due to hepatitis $\mathrm{C}$. $\mathrm{CKD}=$ chronic kidney disease. $\mathrm{CMP}=$ cardiomyopathy and myocarditis. HTN HD=hypertensive heart disease. IHD=ischaemic heart disease. Stroke $=$ cerebrovascular disease. Diabetes=diabetes mellitus. Alcohol=alcohol use disorders. Anxiety=anxiety disorders. Bipolar=bipolar disorder. Drugs=drug use disorders. Depression=depressive disorders. Other MSK=other musculoskeletal disorders. Back and neck=low back and neck pain. Breast $\mathrm{C}=$ breast cancer. Colorectal $\mathrm{C}=$ colon and rectum cancer. Liver $\mathrm{C}=$ liver cancer. Lung $\mathrm{C}=$ tracheal, bronchus, and lung cancer.

Alzheimer's=Alzheimer disease and other dementias. $\mathrm{COPD}=$ chronic obstructive pulmonary disease. Skin=skin and subcutaneous diseases. Fire=fire, heat, and hot substances.

Mech=exposure to mechanical forces. War=collective violence and legal intervention.

Violence=interpersonal violence. Road inj=road injuries. Haemog=haemoglobinopathies and haemolytic anaemias. ILD=interstitial lung disease and pulmonary sarcoidosis.

Disaster=exposure to forces of nature. 


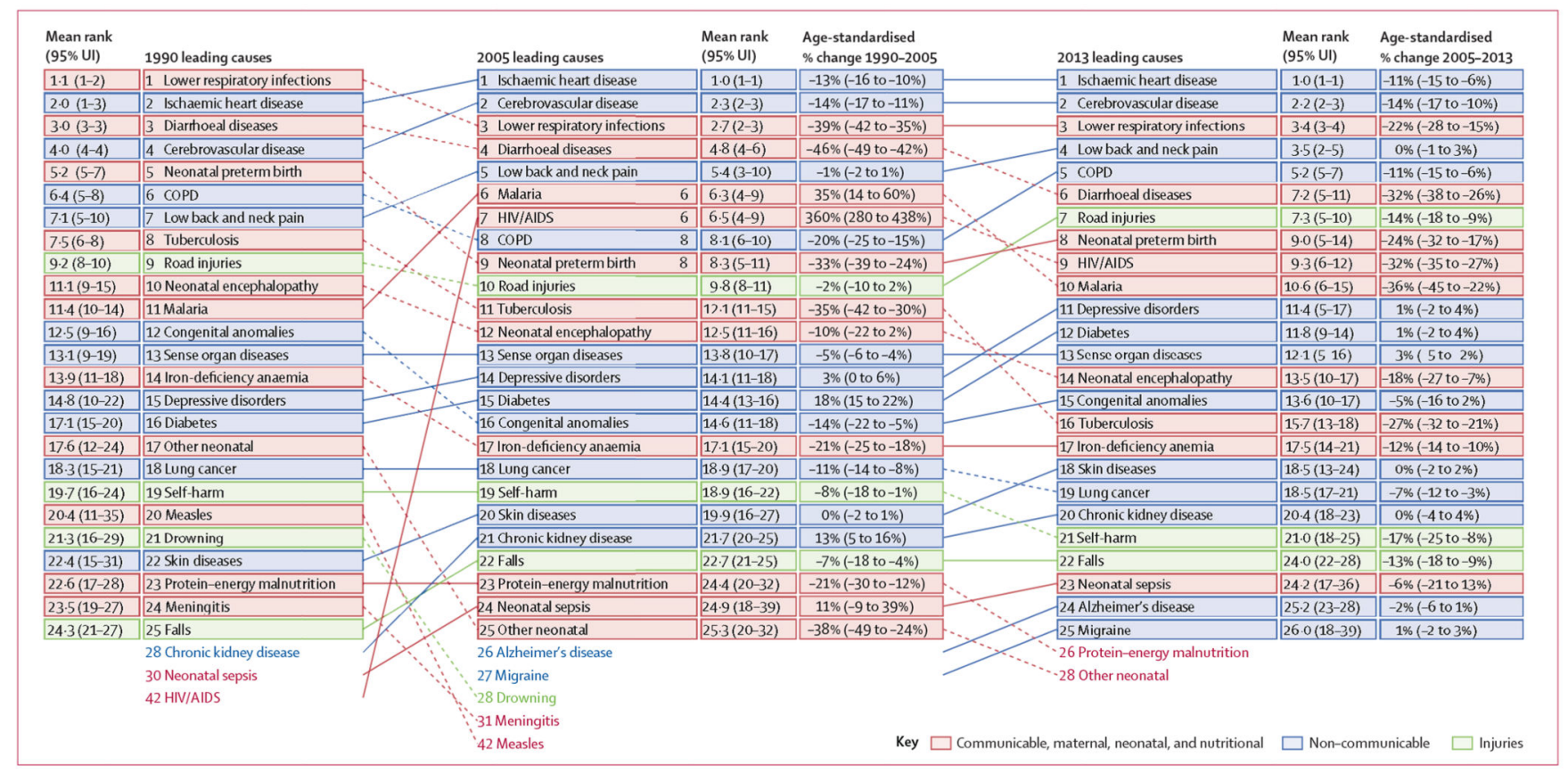

Figure 4. 25 most common GBD level 3 causes of global DALYs for both sexes combined, 1990, 2005, and 2013, with age-standardised median percentage change

Ranks are based on the number of DALYs. 95\% UIs for mean rank are from 1000 draws of

DALYs. Communicable, maternal, neonatal, and nutritional disorders causes are shown in red, non-communicable causes in blue, and injuries in green. DALY=disability-adjusted lifeyears. $\mathrm{GBD}=\mathrm{Global}$ Burden of Disease. $\mathrm{UI}=$ uncertainty interval. $\mathrm{COPD}=$ chronic obstructive pulmonary disease. 


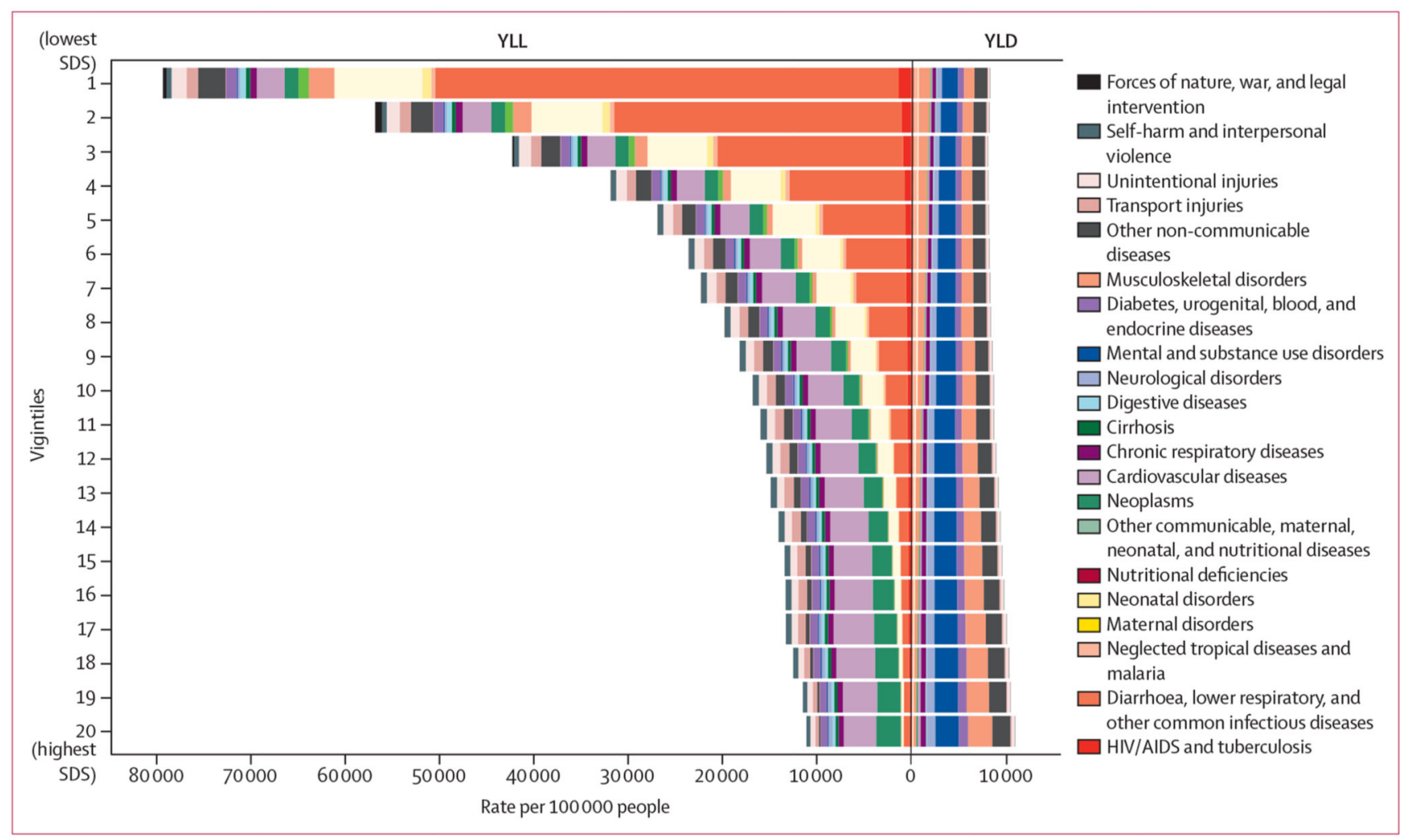

Figure 5. YLL and YLD cause composition of DALY rates by sociodemographic status vigintile The epidemiological transition based on predicted YLL and YLD rates per 100000 people as a function of the level of sociodemographic status by vigintile and broken down into GBD level 2 causes. These predicted levels control for variation explained by year and country. YLL= years of life lost. YLD=years lived with disability. GBD=Global Burden of Disease. 


\begin{tabular}{|c|c|c|c|c|c|c|c|c|c|c|}
\hline & 1 & 2 & 3 & 4 & 5 & 6 & 7 & 8 & 9 & 10 \\
\hline Global & IHD & LRI & Stroke & Back \& neck & Road inj & Diarrhoea & COPD & NN preterm & HIV/AIDS & Malaria \\
\hline Developed & IHD & Back \& neck & Stroke & Lung C & Depression & COPD & Sense & Diabetes & Alzheimer's & Falls \\
\hline Developing & IHD & LRI & Stroke & Back\& neck & Diarrhoea & NN preterm & HIVIAIDS & Road inj & Malaria & COPD \\
\hline High-income & Back \& neck & IHD & Stroke & $\begin{array}{l}\text { Lung } C \\
\end{array}$ & COPD & Depression & Diabetes & \begin{tabular}{|l} 
Alzheimer's \\
\end{tabular} & Sense & Other MSK \\
\hline Australasia & Back \& neck & IHD & Depression & Other MSK & COPD & Alzheimer's & Lung C & Stroke & Diabetes & Sense \\
\hline Australia & Back \& neck & IHD & Depression & Other MSK & COPD & Alzheimer's & Lung C & Stroke & Diabetes & Sense \\
\hline New Zealand & Back \& neck & IHD & COPD & Depression & Alzheimer's & Lung C & Stroke & Diabetes & Sense & Other MSK \\
\hline High-income Asia Pacific & Back \& neck & Stroke & IHD & Diabetes & Lung $C$ & Self-harm & Other MSK & LRI & Sense & Depression \\
\hline Brunei & Back \& neck & IHD & Diabetes & Stroke & Depression & Road inj & Congenital & Skin & Iron & Other MSK \\
\hline Japan & Back \& neck & Stroke & IHD & IRI & Lung C & Other MSK & Diabetes & Sense & Self-harm & Depression \\
\hline Singapore & IHD & Depression & Diabetes & Back \& neck & LRI & Stroke & Sense & Other MSK & Lung $C$ & Skin \\
\hline South Korea & Back \& neck & Stroke & Diabetes & Self-harm & IHD & Liver C & Lung C & Other MSK & Stomach C & Migraine \\
\hline High-income North America & IHD & Back \& neck & COPD & Lung C & Depression & Diabetes & Alzheimer's & Other MSK & Stroke & Sense \\
\hline Canada & Back \& neck & IHD & Lung $\mathrm{C}$ & Other MSK & Alzheimer's & Diabetes & Sense & Depression & COPD & Stroke \\
\hline USA & IHD & Back \& neck & COPD & Lung $C$ & Depression & Diabetes & Alzheimer's & Other MSK & Stroke & Sense \\
\hline Southern Latin America & IHD & Back \& neck & COPD & Stroke & Depression & LRI & Road inj & Congenital & Diabetes & Skin \\
\hline Argentina & IHD & COPD & Back \& neck & Stroke & LRI & Depression & Road inj & Congenital & Diabetes & Skin \\
\hline Chile & Back \& neck & IHD & Stroke & Depression & COPD & Road inj & Skin & Congenital & Anxiety & CKD \\
\hline Uruguay & IHD & Stroke & Back \& neck & COPD & Alzheimer's & Lung C & Depression & Road inj & Sense & LRI \\
\hline Western Europe & Back \& neck & HHD & Stroke & Lung $C$ & Alzheimer's & Sense & $\begin{array}{l}\text { Falls } \\
\end{array}$ & Depression & COPD & Diabetes \\
\hline Andorra & Back \& neck & IHD & Stroke & Sense & Lung $C$ & Depression & Alzheimer's & \begin{tabular}{|l} 
Falls \\
\end{tabular} & COPD & Skin \\
\hline Austria & IHD & Back \& neck & Stroke & Alzheimer's & Lung C & Sense & Falls & Depression & Diabetes & COPD \\
\hline Belgium & Back \& neck & IHD & Lung C & COPD & Alzheimer's & Stroke & Falls & Road inj & Sense & Self-harm \\
\hline Cyprus & Back \& neck & IHD & Diabetes & Depression & Stroke & Sense & Alzheimer's & Skin & Lung C & CKD \\
\hline Denmark & Back \& neck & IHD & COPD & Stroke & Lung C & Alzheimer's & Falls & \begin{tabular}{|l} 
Depression \\
\end{tabular} & Skin & Colorect C \\
\hline Finland & IHD & Back \& neck & Falls & Alzheimer's & Stroke & Depression & Diabetes & Sense & Lung C & Self-harm \\
\hline France & Back \& neck & IHD & Lung $C$ & Falls & Depression & Sense & Stroke & Skin & Alzheimer's & Self-harm \\
\hline Germany & Back \& neck & IHD & Stroke & Sense & Alzheimer's & Lung C & Diabetes & COPD & Falls & CKD \\
\hline Greece & IHD & Back \& neck & Stroke & Lung $C$ & COPD & Alzheimer's & Sense & Depression & CKD & Falls \\
\hline Iceland & Back \& neck & IHD & Skin & COPD & Sense & Alzheimer's & Lung C & Depression & Diabetes & Stroke \\
\hline Ireland & IHD & Back \& neck & Depression & COPD & Lung C & Stroke & 5kin & Sense & Falls & Diabetes \\
\hline israel & Back \& neck & IHD & Depression & Diabetes & Alzheimer's & CKD & Skin & Sense & COPD & Lung C \\
\hline Italy & Back \& neck & IHD & Alzheimer's & Sense & Stroke & Lung C & Falls & Depression & Migraine & COPD \\
\hline Luxembourg & Back \& neck & IHD & Stroke & Lung C & COPD & Depression & Migraine & Sense & Falls & Diabetes \\
\hline Malta & Back \& neck & IHD & Diabetes & Stroke & Depression & Sense & Lung $C$ & Falls & CKD & COPD \\
\hline Netherlands & Back \& neck & IHD & Lung C & COPD & Skin & Stroke & Diabetes & Sense & Depression & Alzheimer's \\
\hline Norway & Back \& neck & IHD & Alzheimer's & Stroke & Lung C & Falls & COPD & Anxiety & Depression & Skin \\
\hline Portugal & Back \& neck & Stroke & IHD & Diabetes & Depression & Alzheimer's & Sense & Lung C & CKD & COPD \\
\hline Spain & Back \& neck & IHD & Diabetes & Stroke & COPD & Alzheimer's & Depression & Lung C & Sense & Falls \\
\hline Sweden & Back \& neck & IHD & Stroke & Falls & COPD & Depression & Sense & Alzheimer's & Lung C & Skin \\
\hline Switzerland & Back \& neck & IHD & Falls & COPD & Alzheimer's & Stroke & Sense & Lung C & Depression & Skin \\
\hline UK & Back \& neck & IHD & Stroke & COPD & Lung C & Alzheimer's & Sense & Depression & Falls & Skin \\
\hline England & Back \& neck & IHD & Stroke & COPD & Lung $\mathrm{C}$ & Alzheimer's & Sense & Depression & Falls & Skin \\
\hline Northern Ireland & IHD & Back \& neck & Depression & Lung $C$ & Stroke & COPD & Alzheimer's & Falls & Skin & Sense \\
\hline Scotland & Back \& neck & IHD & Lung C & Stroke & COPD & Alzheimer's & Sense & Diabetes & Falls & Skin \\
\hline Wales & Back \& neck & IHD & Stroke & COPD & Lung C & Alzheimer's & Depression & Sense & Falls & Diabetes \\
\hline Central Europe, eastern Europe, and central Asia & IHD & Stroke & Back \& neck & $\mid$ LRI & Self-harm & Depression & \begin{tabular}{|l|} 
Sense \\
\end{tabular} & Lung C & Road inj & \begin{tabular}{|l|} 
COPD \\
\end{tabular} \\
\hline Central Asia & IHD & LRI & \begin{tabular}{|l|} 
Stroke \\
\end{tabular} & NN enceph & Back \& neck & Congenital & Road inj & Depression & \begin{tabular}{|l|} 
Diabetes \\
\end{tabular} & NN preterm \\
\hline Armenia & IHD & Stroke & Diabetes & Back \& neck & Lung $C$ & Depression & Sense & Road inj & Congenital & COPD \\
\hline Azerbaijan & IHD & LRI & Stroke & Back \& neck & Diabetes & NN enceph & Depression & Congenital & Road inj & Sense \\
\hline Georgia & IHD & Stroke & COPD & Diabetes & Back \& neck & Sense & Depression & Falls & Alzheimer's & Road inj \\
\hline Kazakhstan & IHD & Stroke & Self-harm & Back \& neck & Road inj & Congenital & LRI & COPD & NN enceph & Depression \\
\hline Kyrgyzstan & IHD & Stroke & LRI & NN enceph & NN preterm & Congenital & Back \& neck & Road inj & COPD & Depression \\
\hline Mongolia & IHD & Stroke & LRI & Liver C & NN enceph & Congenital & Self-harm & Road inj & NN preterm & Back \& neck \\
\hline Tajikistan & LRI & IHD & NN preterm & Diarrhoea & NN enceph & Congenital & Stroke & Back \& neck & Depression & Drown \\
\hline Turkmenistan & IHD & LRI & Stroke & NN enceph & Diarrhoea & Back \& neck & Congenital & NN preterm & Depression & Diabetes \\
\hline Uzbekistan & IHD & LRI & Stroke & NN enceph & Back \& neck & Road inj & Depression & Diabetes & Congenital & HTN HD \\
\hline Central Europe & IHD & Stroke & Back \& neck & Lung $C$ & Falls & COPD & Sense & Diabetes & Depression & Alzheimer's \\
\hline Albania & IHD & Stroke & Back \& neck & Depression & LRI & Falls & Lung C & Sense & COPD & Diabetes \\
\hline Bosnia and Herzegovina & IHD & Stroke & Back \& neck & Diabetes & Lung $C$ & CMP & COPD & Sense & Depression & Falls \\
\hline Bulgaria & IHD & Stroke & Back \& neck & COPD & Diabetes & HTN HD & Falls & Lung C & Sense & Alzheimer's \\
\hline Croatia & IHD & Stroke & Back \& neck & Lung $C$ & Sense & COPD & Diabetes & Alzheimer's & Depression & Falls \\
\hline Czech Republic & IHD & Back \& neck & Stroke & Falls & Lung C & Sense & Diabetes & COPD & Depression & Alzheimer's \\
\hline Hungary & IHD & Stroke & Back \& neck & Lung C & Falls & COPD & Sense & Diabetes & Colorect C & Depression \\
\hline Macedonia & Stroke & IHD & Back \& neck & Diabetes & Lung C & Depression & Sense & Falls & COPD & CKD \\
\hline Montenegro & IHD & Stroke & Back \& neck & Lung C & Diabetes & Sense & Falls & Depression & Self-harm & CKD \\
\hline Poland & IHD & Stroke & Back \& neck & Lung $\mathrm{C}$ & Falls & COPD & Sense & Depression & Diabetes & Self-harm \\
\hline Romania & IHD & Stroke & Back \& neck & Falls & Lung $\mathrm{C}$ & Sense & Depression & COPD & Diabetes & Alzheimer's \\
\hline Serbia & IHD & Stroke & Back \& neck & CMP & Lung $C$ & Diabetes & Sense & COPD & Depression & Alzheimer's \\
\hline Slovakia & IHD & Back \& neck & Stroke & Falls & Lung C & Sense & Depression & Diabetes & Colorect C & COPD \\
\hline Slovenia & Back \& neck & IHD & Falls & Stroke & Lung C & Sense & Depression & COPD & Alzheimer's & Diabetes \\
\hline Eastern Europe & IHD & Stroke & Back \& neck & Self-harm & CMP & Depression & Sense & Alcohol & Lung C & Road inj \\
\hline
\end{tabular}




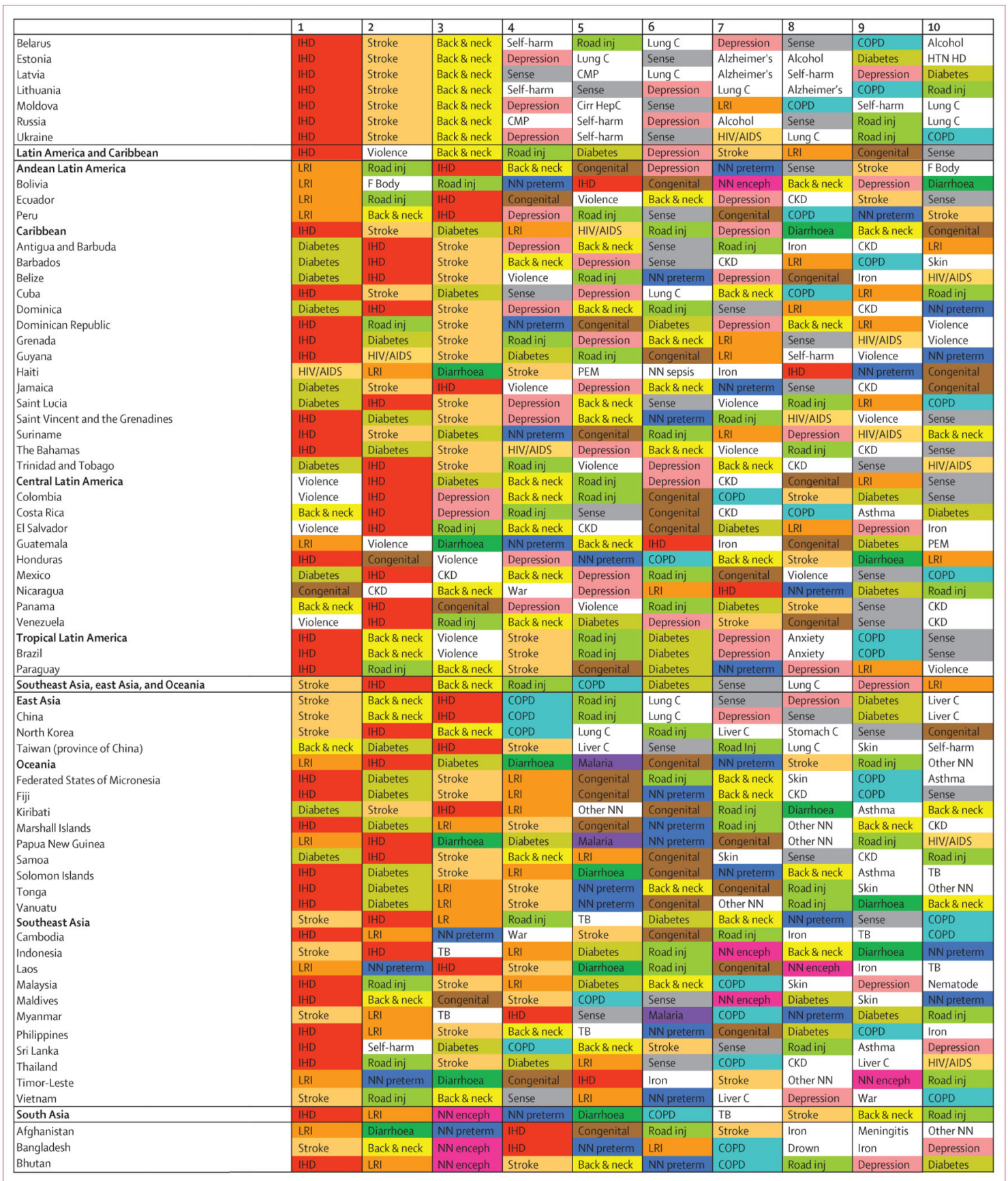




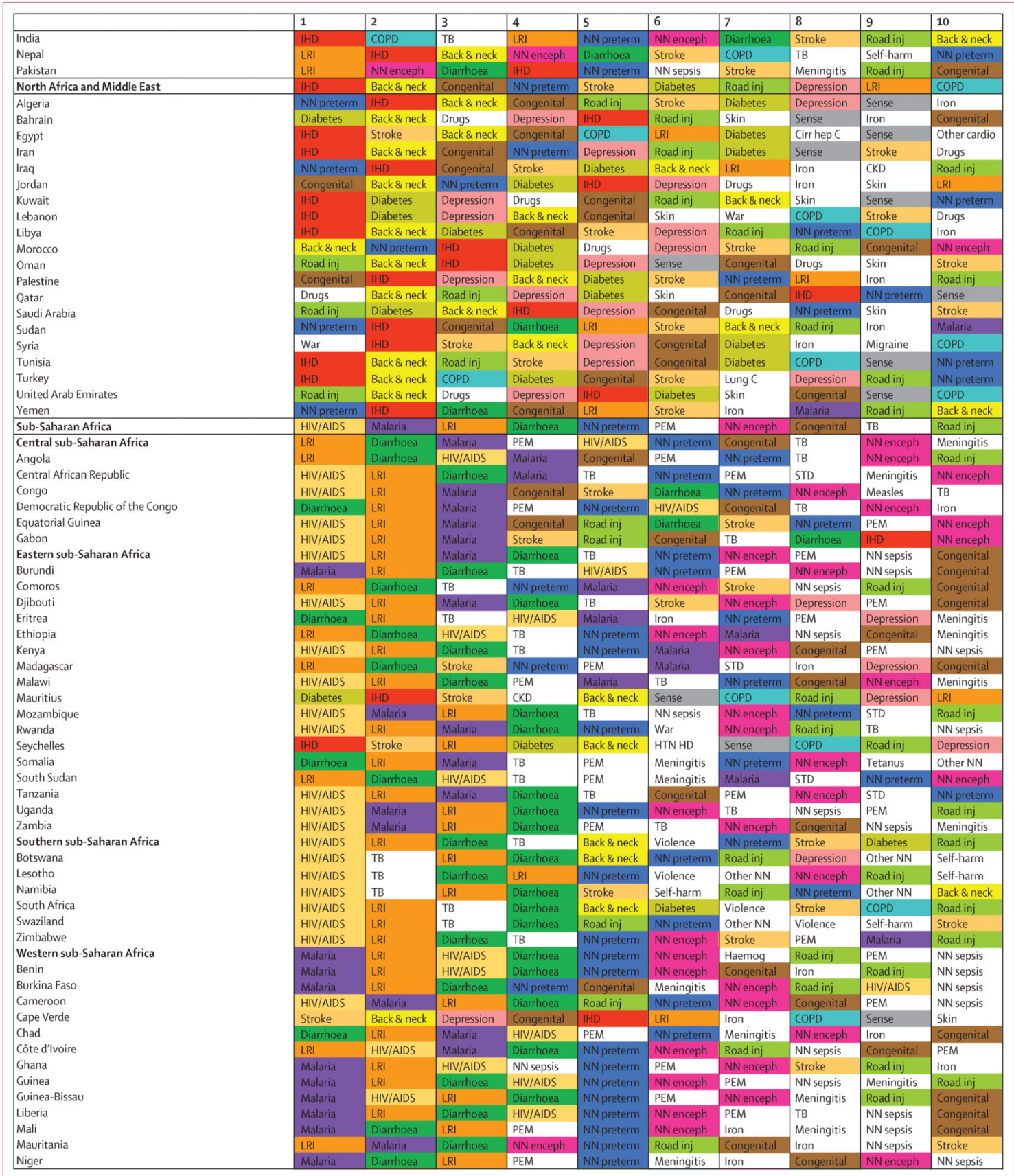




\begin{tabular}{|c|c|c|c|c|c|c|c|c|c|c|}
\hline & 1 & 2 & 3 & 4 & 5 & 6 & 7 & 8 & 9 & 10 \\
\hline Nigeria & \multirow{6}{*}{$\begin{array}{l}\text { Malaria } \\
\text { Malaria } \\
\text { Malaria } \\
\text { Malaria } \\
\text { Malaria } \\
\text { Malaria } \\
\end{array}$} & \multirow{6}{*}{$\begin{array}{l}\text { LRI } \\
\text { LRI } \\
\text { LRI } \\
\text { LRI } \\
\text { LRI } \\
\text { LRI }\end{array}$} & \multirow{2}{*}{$\begin{array}{l}\text { HIV/AIDS } \\
\text { Stroke }\end{array}$} & Haemog & Road inj & NN preterm & NN enceph & Diarrhoea & \multirow{2}{*}{$\begin{array}{l}\text { PEM } \\
\text { PEM }\end{array}$} & NN sepsis \\
\hline São Tomé and Príncipe & & & & \multirow{2}{*}{$\begin{array}{l}\text { NN preterm } \\
\text { NN preterm }\end{array}$} & \multirow{2}{*}{$\begin{array}{l}\text { Congenital } \\
\text { NN enceph }\end{array}$} & NN enceph & \multirow{2}{*}{$\begin{array}{l}\text { Iron } \\
\text { NN sepsis }\end{array}$} & \multirow{2}{*}{$\begin{array}{l}\text { NN sepsis } \\
\text { HIV/AIDS }\end{array}$} & & Diarrhoea \\
\hline Senegal & & & Diarrhoea & & & Iron & & & Congenital & Road inj \\
\hline Sierra Leone & & & HIV/AIDS & PEM & NN preterm & Diarrhoea & NN enceph & Congenital & NN sepsis & Haemog \\
\hline The Gambia & & & Diarrhoea & Congenital & NN preterm & HIV/AIDS & NN sepsis & NN enceph & Road inj & PEM \\
\hline Togo & & & HIV/AIDS & Diarrhoea & NN preterm & NN enceph & Congenital & PEM & NN sepsis & Haemog \\
\hline Ischaemic heart disease & IHD & Road & & & Road inj & Depressive dis & orders & & & Depression \\
\hline Lower respiratory infections & LRI & Diarrl & eases & & Diarrhoea & Neonatal ence & phalopathy d & e to birth asph & xia and trauma & NN enceph \\
\hline Cerebrovascular disease & Stroke & Chror & stive pulmo & y disease & COPD & Congenital an & omalies & & & Congenital \\
\hline HIV/AIDS & HIV/AIDS & Prete & complication & & NN preterm & Diabetes melli & & & & Diabetes \\
\hline Low back and neck pain & Back \& neck & Malar & & & Malaria & Sense organd & seases & & & Sense \\
\hline
\end{tabular}

Figure 6. Ten most common GBD level 3 causes of DALYs for 188 countries in 2013

The 15 most common global causes of DALYs are coloured. Alcohol=alcohol use disorders.

Alzheimer=Alzheimer's disease and other dementias. Anxiety=anxiety disorders. Back and neck=low back and neck pain. Cirr Hep $\mathrm{C}=$ cirrhosis due to hepatitis $\mathrm{C}$. $\mathrm{CKD}=$ chronic

kidney disease. $\mathrm{CMP}=$ cardiomyopathy and myocarditis. Colorect $\mathrm{C}=$ colon and rectum cancer. Congenital $=$ congenital disorders. $\mathrm{COPD}=$ chronic obstructive pulmonary disease. Depression=depressive disorders. Diabetes=diabetes mellitus. Diarrhoea=diarrhoeal diseases. Drown=drowning. Drugs=drug use disorders. F Body=foreign body.

Haemog=haemoglobinopathies and haemolytic anaemias. HTN HD=hypertensive heart disease. $\mathrm{IHD}=$ ischaemic heart disease. Iron=iron-deficiency anaemia. Liver $\mathrm{C}=$ liver cancer. LRI=lower respiratory infections. Lung $\mathrm{C}=$ tracheal, bronchus, and lung cancer.

Nematode=intestinal nematode infections. $\mathrm{NN}$ enceph=neonatal encephalopathy due to birth asphyxia and trauma. NN preterm=preterm birth complications. NN sepsis=neonatal sepsis and other neonatal infections. Other cardio=other cardiovascular and circulatory diseases. Other $\mathrm{MSK}=$ other musculoskeletal disorders. Other $\mathrm{NN}=$ other neonatal disorders. $\mathrm{PEM}=$ protein-energy malnutrition. Road inj=road injuries. Sense=sense organ diseases. Skin=skin and subcutaneous diseases. STD=sexually transmitted diseases excluding HIV. Stomach $\mathrm{C}=$ stomach cancer. Stroke=cerebrovascular disease. $\mathrm{TB}=$ tuberculosis. Violence=interpersonal violence. War=collective violence and legal intervention. DALY=disability-adjusted life-years. GBD=Global Burden of Disease. 
Table 1

Global all-age DALYs and age-standardised DALYs for 306 causes in 2005 and 2013 with percentage change

\begin{tabular}{|c|c|c|c|c|c|c|}
\hline & \multicolumn{3}{|c|}{ All ages DALYs (thousands) ${ }^{*}$} & \multicolumn{3}{|c|}{ Age-standardised DALYs (per 100000$)^{*}$} \\
\hline & 2005 & 2013 & Percentage change & 2005 & 2013 & Percentage change \\
\hline All causes & $\begin{array}{c}2513 \\
239 \cdot 2(2 \\
331974 \cdot 5 \\
\text { to } 2717 \\
184 \cdot 5)\end{array}$ & $\begin{array}{c}2449 \\
810 \cdot 0(2 \\
234094 \cdot 6 \\
\text { to } 2675 \\
167 \cdot 6)\end{array}$ & $-2 \cdot 5(-4 \cdot 7 \text { to }-0 \cdot 3)^{*}$ & $\begin{array}{c}41072 \cdot 6 \\
(38101 \cdot 1 \\
\text { to } 44 \\
409 \cdot 9)\end{array}$ & $\begin{array}{c}35523 \cdot 9 \\
(32416 \cdot 1 \\
\text { to } 38 \\
774 \cdot 8)\end{array}$ & $\begin{array}{c}-13 \cdot 5(-15 \cdot 3 \text { to } \\
-11 \cdot 6)^{*}\end{array}$ \\
\hline $\begin{array}{l}\text { Communicable, maternal, neonatal, } \\
\text { and nutritional diseases }\end{array}$ & $\begin{array}{c}943358 \cdot 6 \\
(903 \\
197 \cdot 4 \text { to } \\
985 \\
632 \cdot 0)\end{array}$ & $\begin{array}{c}769288 \cdot 8 \\
(725 \\
481 \cdot 2 \text { to } \\
814 \\
936 \cdot 0)\end{array}$ & $\begin{array}{c}-18 \cdot 5(-21 \cdot 2 \text { to } \\
-15 \cdot 8)^{*}\end{array}$ & $\begin{array}{c}14031 \cdot 9 \\
(13434 \cdot 2 \\
\text { to } 14 \\
662 \cdot 0)\end{array}$ & $\begin{array}{c}10606 \cdot 9 \\
(10004 \cdot 2 \\
\text { to } 11 \\
234 \cdot 3)\end{array}$ & $\begin{array}{c}-24 \cdot 4(-26 \cdot 9 \text { to } \\
-21 \cdot 9)^{*}\end{array}$ \\
\hline HIV/AIDS and tuberculosis & $\begin{array}{c}150304 \cdot 0 \\
(141 \\
828 \cdot 2 \text { to } \\
159 \\
539 \cdot 6)\end{array}$ & $\begin{array}{c}119179 \cdot 6 \\
(112 \\
497 \cdot 7 \text { to } \\
127 \\
584 \cdot 9)\end{array}$ & $\begin{array}{c}-20 \cdot 8(-24 \cdot 4 \text { to } \\
-16 \cdot 5)^{*}\end{array}$ & $\begin{array}{c}2344 \cdot 3 \\
(2214 \cdot 0 \\
\text { to } \\
2489 \cdot 5)\end{array}$ & $\begin{array}{c}1656 \cdot 0 \\
(1563 \cdot 5 \\
\text { to } \\
1774 \cdot 5)\end{array}$ & $\begin{array}{c}-29 \cdot 5(-32 \cdot 6 \text { to } \\
-25 \cdot 6)^{*}\end{array}$ \\
\hline Tuberculosis & $\begin{array}{c}59600 \cdot 6 \\
(53405 \cdot 7 \\
\text { to } 64 \\
094 \cdot 8)\end{array}$ & $\begin{array}{c}49816 \cdot 2 \\
(44744 \cdot 3 \\
\text { to } 54 \\
313 \cdot 4)\end{array}$ & $\begin{array}{c}-16 \cdot 4(-22 \cdot 9 \text { to } \\
-9 \cdot 4)^{*}\end{array}$ & $\begin{array}{c}964 \cdot 1 \\
(866 \cdot 4 \text { to } \\
1035 \cdot 6)\end{array}$ & $\begin{array}{c}706 \cdot 9 \\
(635 \cdot 9 \text { to } \\
771 \cdot 7)\end{array}$ & $\begin{array}{c}-26 \cdot 7(-32 \cdot 3 \text { to } \\
-20 \cdot 5)^{*}\end{array}$ \\
\hline HIV/AIDS & $\begin{array}{c}90703 \cdot 4 \\
(83401 \cdot 7 \\
\text { to } 99 \\
132 \cdot 8)\end{array}$ & $\begin{array}{c}69363 \cdot 4 \\
(64972 \cdot 5 \\
\text { to } 76 \\
330 \cdot 2)\end{array}$ & $\begin{array}{c}-23.9(-28.1 \text { to } \\
-18 \cdot 5)^{*}\end{array}$ & $\begin{array}{c}1380 \cdot 2 \\
(1269 \cdot 6 \\
\text { to } \\
1507 \cdot 9)\end{array}$ & $\begin{array}{c}949 \cdot 1 \\
(890 \cdot 0 \text { to } \\
1045 \cdot 1)\end{array}$ & $\begin{array}{c}-31 \cdot 5(-35 \cdot 3 \text { to } \\
-26 \cdot 7)^{*}\end{array}$ \\
\hline $\begin{array}{l}\text { HIV/AIDS resulting in } \\
\text { mycobacterial infection }\end{array}$ & $\begin{array}{c}6573 \cdot 9 \\
(5371 \cdot 5 \text { to } \\
8050 \cdot 8)\end{array}$ & $\begin{array}{c}4303 \cdot 1 \\
(3496 \cdot 7 \text { to } \\
5323 \cdot 6)\end{array}$ & $\begin{array}{c}-34.8(-38.8 \text { to } \\
-29 \cdot 4)^{*}\end{array}$ & $\begin{array}{l}100 \cdot 3 \\
(82 \cdot 0 \text { to } \\
122 \cdot 8)\end{array}$ & $\begin{array}{c}58.9 \\
(47.9 \text { to } \\
72.9)\end{array}$ & $\begin{array}{c}-41 \cdot 5(-45 \cdot 1 \text { to } \\
-36 \cdot 7)^{*}\end{array}$ \\
\hline $\begin{array}{l}\text { HIV/AIDS resulting in other } \\
\text { diseases }\end{array}$ & $\begin{array}{c}84129 \cdot 5 \\
(77516 \cdot 6 \\
\text { to } 92 \\
482 \cdot 2)\end{array}$ & $\begin{array}{c}65060 \cdot 3 \\
(60939 \cdot 8 \\
\text { to } 71 \\
903 \cdot 6)\end{array}$ & $\begin{array}{c}-23 \cdot 0(-27 \cdot 3 \text { to } \\
-17 \cdot 6)^{*}\end{array}$ & $\begin{array}{c}1279 \cdot 9 \\
(1179 \cdot 5 \\
\text { to } \\
1404 \cdot 1)\end{array}$ & $\begin{array}{c}890 \cdot 2 \\
(834 \cdot 3 \text { to } \\
984 \cdot 9)\end{array}$ & $\begin{array}{c}-30 \cdot 7(-34 \cdot 6 \text { to } \\
-25 \cdot 9)^{*}\end{array}$ \\
\hline $\begin{array}{l}\text { Diarrhoea, lower respiratory, and } \\
\text { other common infectious diseases }\end{array}$ & $\begin{array}{c}316908 \cdot 8 \\
(298 \\
964 \cdot 3 \text { to } \\
335 \\
711 \cdot 2)\end{array}$ & $\begin{array}{c}249855 \cdot 1 \\
(231 \\
222 \cdot 1 \text { to } \\
269 \\
625 \cdot 3)\end{array}$ & $\begin{array}{c}-21 \cdot 1(-26 \cdot 0 \text { to } \\
-16 \cdot 4)^{*}\end{array}$ & $\begin{array}{c}4762 \cdot 3 \\
(4499 \cdot 7 \\
\text { to } \\
5038 \cdot 0)\end{array}$ & $\begin{array}{c}3488 \cdot 6 \\
(3231 \cdot 6 \\
\text { to } \\
3760 \cdot 2)\end{array}$ & $\begin{array}{c}-26 \cdot 7(-31 \cdot 2 \text { to } \\
-22 \cdot 4)^{*}\end{array}$ \\
\hline Diarrhoeal diseases & $\begin{array}{c}99453 \cdot 9 \\
(90724 \cdot 0 \\
\text { to } 108 \\
320 \cdot 9)\end{array}$ & $\begin{array}{c}72796 \cdot 6 \\
(65452 \cdot 9 \\
\text { to } 80 \\
756 \cdot 7)\end{array}$ & $\begin{array}{c}-26.9(-33.5 \text { to } \\
-19.7)^{*}\end{array}$ & $\begin{array}{c}1497 \cdot 5 \\
(1370 \cdot 2 \\
\text { to } \\
1625 \cdot 7)\end{array}$ & $\begin{array}{l}1015 \cdot 5 \\
(915 \cdot 5 \text { to } \\
1126 \cdot 3)\end{array}$ & $\begin{array}{c}-32 \cdot 3(-38 \cdot 4 \text { to } \\
-25 \cdot 8)^{*}\end{array}$ \\
\hline Intestinal infectious diseases & $\begin{array}{l}17538 \cdot 4 \\
(9936 \cdot 1 \text { to } \\
28497 \cdot 3)\end{array}$ & $\begin{array}{l}15376 \cdot 5 \\
(8627 \cdot 8 \text { to } \\
24958 \cdot 9)\end{array}$ & $-12 \cdot 6(-22 \cdot 2$ to $1 \cdot 0)$ & $\begin{array}{c}252 \cdot 9 \\
(143 \cdot 5 \text { to } \\
410 \cdot 7)\end{array}$ & $\begin{array}{c}210 \cdot 7 \\
(118 \cdot 4 \text { to } \\
341 \cdot 5)\end{array}$ & $\begin{array}{c}-17 \cdot 0(-26 \cdot 2 \text { to } \\
-4 \cdot 2)^{*}\end{array}$ \\
\hline Typhoid fever & $\begin{array}{l}12863 \cdot 1 \\
(7058 \cdot 4 \text { to } \\
21368 \cdot 4)\end{array}$ & $\begin{array}{c}11127 \cdot 8 \\
(6013 \cdot 6 \text { to } \\
18314 \cdot 8)\end{array}$ & $-13 \cdot 7(-24 \cdot 6$ to $1 \cdot 0)$ & $\begin{array}{c}185 \cdot 4 \\
(101 \cdot 8 \text { to } \\
307 \cdot 6)\end{array}$ & $\begin{array}{l}152 \cdot 5 \\
(82 \cdot 6 \text { to } \\
250 \cdot 5)\end{array}$ & $\begin{array}{c}-17 \cdot 9(-28 \cdot 3 \text { to } \\
-4 \cdot 0)^{*}\end{array}$ \\
\hline Paratyphoid fever & $\begin{array}{c}4150 \cdot 1 \\
(2271 \cdot 2 \text { to } \\
6940 \cdot 7)\end{array}$ & $\begin{array}{c}3820 \cdot 5 \\
(2084 \cdot 1 \text { to } \\
6493 \cdot 0)\end{array}$ & $-8 \cdot 0(-25 \cdot 2$ to $12 \cdot 5)$ & $\begin{array}{c}59 \cdot 8 \\
(32 \cdot 8 \text { to } \\
100 \cdot 1)\end{array}$ & $\begin{array}{c}52 \cdot 3 \\
(28 \cdot 6 \text { to } \\
89 \cdot 0)\end{array}$ & $-12 \cdot 5(-28 \cdot 8$ to $6 \cdot 7)$ \\
\hline $\begin{array}{l}\text { Other intestinal infectious } \\
\text { diseases }\end{array}$ & $\begin{array}{l}525 \cdot 2 \\
(460 \cdot 0 \text { to } \\
598 \cdot 7)\end{array}$ & $\begin{array}{c}428 \cdot 3 \\
(373 \cdot 2 \text { to } \\
486 \cdot 9)\end{array}$ & $\begin{array}{c}-18 \cdot 4(-25 \cdot 3 \text { to } \\
-11 \cdot 4)^{*}\end{array}$ & $\begin{array}{l}7 \cdot 7(6 \cdot 8 \\
\text { to } 8 \cdot 8)\end{array}$ & $\begin{array}{l}5 \cdot 9(5 \cdot 1 \\
\text { to } 6 \cdot 7)\end{array}$ & $\begin{array}{c}-24 \cdot 2(-30 \cdot 6 \text { to } \\
-17 \cdot 7) *\end{array}$ \\
\hline
\end{tabular}




\begin{tabular}{|c|c|c|c|c|c|c|}
\hline & \multicolumn{3}{|c|}{ All ages DALYs (thousands) ${ }^{*}$} & \multicolumn{3}{|c|}{ Age-standardised DALYs (per 100000 ) } \\
\hline & 2005 & 2013 & Percentage change & 2005 & 2013 & Percentage change \\
\hline Lower respiratory infections & $\begin{array}{c}133899 \cdot 9 \\
(124 \\
847 \cdot 7 \text { to } \\
142 \\
498 \cdot 1)\end{array}$ & $\begin{array}{c}113363 \cdot 1 \\
(103 \\
083 \cdot 5 \text { to } \\
122 \\
202 \cdot 1)\end{array}$ & $\begin{array}{c}-15 \cdot 3(-22 \cdot 1 \text { to } \\
-8 \cdot 2)^{*}\end{array}$ & $\begin{array}{c}2043 \cdot 4 \\
(1912 \cdot 2 \\
\text { to } \\
2169 \cdot 8)\end{array}$ & $\begin{array}{c}1599 \cdot 1 \\
(1451 \cdot 9 \\
\text { to } \\
1721 \cdot 3)\end{array}$ & $\begin{array}{c}-21.7(-27.8 \text { to } \\
-15 \cdot 5)^{*}\end{array}$ \\
\hline Upper respiratory infections & $\begin{array}{c}2743 \cdot 4 \\
(1630 \cdot 3 \text { to } \\
4451 \cdot 0)\end{array}$ & $\begin{array}{c}3031 \cdot 0 \\
(1757 \cdot 4 \text { to } \\
4934 \cdot 2)\end{array}$ & $10.4(7.3 \text { to } 13.4)^{*}$ & $\begin{array}{c}41 \cdot 3 \\
(24 \cdot 5 \text { to } \\
66 \cdot 8)\end{array}$ & $\begin{array}{c}41.9 \\
(24 \cdot 3 \text { to } \\
68 \cdot 2)\end{array}$ & $1 \cdot 6(-1 \cdot 3$ to $4 \cdot 3)$ \\
\hline Otitis media & $\begin{array}{c}1751 \cdot 5 \\
(1102.5 \text { to } \\
2735 \cdot 2)\end{array}$ & $\begin{array}{c}1806 \cdot 5 \\
(1129 \cdot 8 \text { to } \\
2812 \cdot 2)\end{array}$ & $3.1(0.5 \text { to } 5.7)^{*}$ & $\begin{array}{c}26 \cdot 2 \\
(16 \cdot 5 \text { to } \\
40 \cdot 8)\end{array}$ & $\begin{array}{l}25 \cdot 0 \\
(15 \cdot 6 \text { to } \\
38 \cdot 9)\end{array}$ & $-4.5(-7.0 \text { to }-2.0)^{*}$ \\
\hline Meningitis & $\begin{array}{c}24317 \cdot 0 \\
(21010 \cdot 6 \\
\text { to } 27 \\
891 \cdot 4)\end{array}$ & $\begin{array}{c}21014.9 \\
(17519 \cdot 8 \\
\text { to } 24 \\
328 \cdot 1)\end{array}$ & $\begin{array}{c}-13 \cdot 7(-20 \cdot 8 \text { to } \\
-5 \cdot 5)^{*}\end{array}$ & $\begin{array}{c}358 \cdot 3 \\
(310 \cdot 2 \text { to } \\
410 \cdot 1)\end{array}$ & $\begin{array}{c}288 \cdot 2 \\
(240 \cdot 6 \text { to } \\
333 \cdot 3)\end{array}$ & $\begin{array}{c}-19 \cdot 7(-26 \cdot 3 \text { to } \\
-12 \cdot 1)^{*}\end{array}$ \\
\hline Pneumococcal & $\begin{array}{c}6343 \cdot 8 \\
(5493 \cdot 7 \text { to } \\
7161 \cdot 7)\end{array}$ & $\begin{array}{c}5509 \cdot 2 \\
(4678 \cdot 8 \text { to } \\
6363 \cdot 5)\end{array}$ & $\begin{array}{c}-13 \cdot 3(-20 \cdot 8 \text { to } \\
-3 \cdot 1)^{*}\end{array}$ & $\begin{array}{c}93.9 \\
(81.5 \text { to } \\
105 \cdot 7)\end{array}$ & $\begin{array}{l}75 \cdot 7 \\
(64 \cdot 3 \text { to } \\
87 \cdot 4)\end{array}$ & $\begin{array}{c}-19 \cdot 5(-26 \cdot 5 \text { to } \\
-10 \cdot 4)^{*}\end{array}$ \\
\hline Haemophilus influenzae type B & $\begin{array}{l}6300 \cdot 1 \\
(5188 \cdot 0 \text { to } \\
7431 \cdot 5)\end{array}$ & $\begin{array}{c}5177 \cdot 1 \\
(4196 \cdot 8 \text { to } \\
6211 \cdot 1)\end{array}$ & $\begin{array}{c}-17.8(-27.9 \text { to } \\
-7 \cdot 7)^{*}\end{array}$ & $\begin{array}{c}91 \cdot 6 \\
(75 \cdot 4 \text { to } \\
108 \cdot 1)\end{array}$ & $\begin{array}{l}70 \cdot 7 \\
(57 \cdot 3 \text { to } \\
84 \cdot 7)\end{array}$ & $\begin{array}{c}-22 \cdot 9(-32 \cdot 2 \text { to } \\
-13 \cdot 3)^{*}\end{array}$ \\
\hline Meningococcal & $\begin{array}{c}4733 \cdot 3 \\
(4055 \cdot 1 \text { to } \\
5610 \cdot 2)\end{array}$ & $\begin{array}{c}4314 \cdot 7 \\
(3583 \cdot 0 \text { to } \\
5116 \cdot 6)\end{array}$ & $-8 \cdot 5(-18 \cdot 7$ to $1 \cdot 3)$ & $\begin{array}{c}69.9 \\
(59 \cdot 8 \text { to } \\
82 \cdot 5)\end{array}$ & $\begin{array}{c}59 \cdot 2 \\
(49 \cdot 2 \text { to } \\
70 \cdot 1)\end{array}$ & $\begin{array}{c}-15 \cdot 0(-24 \cdot 4 \text { to } \\
-5 \cdot 8)^{*}\end{array}$ \\
\hline Other meningitis & $\begin{array}{c}6939 \cdot 8 \\
(6097 \cdot 9 \text { to } \\
8066 \cdot 0)\end{array}$ & $\begin{array}{c}6014 \cdot 0 \\
(5084 \cdot 4 \text { to } \\
6954 \cdot 2)\end{array}$ & $\begin{array}{c}-13 \cdot 2(-22 \cdot 2 \text { to } \\
-2 \cdot 9)^{*}\end{array}$ & $\begin{array}{l}102 \cdot 9 \\
(90 \cdot 6 \text { to } \\
119 \cdot 5)\end{array}$ & $\begin{array}{l}82 \cdot 6 \\
(70 \cdot 0 \text { to } \\
95 \cdot 4)\end{array}$ & $\begin{array}{c}-19 \cdot 7(-28.0 \text { to } \\
-10 \cdot 2) *\end{array}$ \\
\hline Encephalitis & $\begin{array}{c}5087 \cdot 0 \\
(4236 \cdot 1 \text { to } \\
6021 \cdot 8)\end{array}$ & $\begin{array}{c}4804 \cdot 2 \\
(4022 \cdot 4 \text { to } \\
5926 \cdot 9)\end{array}$ & $-5 \cdot 3(-19 \cdot 2$ to $9 \cdot 0)$ & $\begin{array}{c}75 \cdot 4 \\
(62 \cdot 9 \text { to } \\
89 \cdot 4)\end{array}$ & $\begin{array}{c}66 \cdot 3 \\
(55 \cdot 6 \text { to } \\
81.9)\end{array}$ & $-11 \cdot 9(-24 \cdot 6$ to $1 \cdot 3)$ \\
\hline Diphtheria & $\begin{array}{c}316 \cdot 5 \\
(153 \cdot 3 \text { to } \\
671 \cdot 8)\end{array}$ & $\begin{array}{l}253 \cdot 6 \\
(126 \cdot 5 \text { to } \\
536 \cdot 1)\end{array}$ & $\begin{array}{c}-17 \cdot 8(-74 \cdot 5 \text { to } \\
124 \cdot 7)\end{array}$ & $\begin{array}{l}4.6(2 \cdot 2 \\
\text { to } 9 \cdot 8)\end{array}$ & $\begin{array}{l}3.5(1.7 \\
\text { to } 7 \cdot 3)\end{array}$ & $\begin{array}{c}-22 \cdot 8(-75 \cdot 9 \text { to } \\
111 \cdot 9)\end{array}$ \\
\hline Whooping cough & $\begin{array}{c}6478 \cdot 7 \\
(2580 \cdot 1 \text { to } \\
12839 \cdot 5)\end{array}$ & $\begin{array}{c}5250 \cdot 9 \\
(2029 \cdot 0 \text { to } \\
11658 \cdot 9)\end{array}$ & $\begin{array}{c}-22 \cdot 4(-71 \cdot 4 \text { to } \\
125 \cdot 5)\end{array}$ & $\begin{array}{c}93 \cdot 9 \\
(37 \cdot 4 \text { to } \\
186 \cdot 1)\end{array}$ & $\begin{array}{c}71.5 \\
(27 \cdot 6 \text { to } \\
158 \cdot 8)\end{array}$ & $\begin{array}{c}-27 \cdot 0(-73 \cdot 1 \text { to } \\
112 \cdot 1)\end{array}$ \\
\hline Tetanus & $\begin{array}{c}7223 \cdot 6 \\
(4402 \cdot 4 \text { to } \\
8704 \cdot 6)\end{array}$ & $\begin{array}{c}3654 \cdot 7 \\
(2312 \cdot 7 \text { to } \\
4911 \cdot 4)\end{array}$ & $\begin{array}{c}-49.8(-58.0 \text { to } \\
-36.8)^{*}\end{array}$ & $\begin{array}{l}105 \cdot 1 \\
(64 \cdot 5 \text { to } \\
126 \cdot 3)\end{array}$ & $\begin{array}{c}50 \cdot 2 \\
(32 \cdot 0 \text { to } \\
67 \cdot 5)\end{array}$ & $\begin{array}{c}-52 \cdot 6(-60 \cdot 2 \text { to } \\
-40 \cdot 5)^{*}\end{array}$ \\
\hline Measles & $\begin{array}{l}17635.2 \\
(9981 \cdot 3 \text { to } \\
28573.5)\end{array}$ & $\begin{array}{c}8015 \cdot 1 \\
(4077 \cdot 1 \text { to } \\
14458 \cdot 0)\end{array}$ & $\begin{array}{c}-55.9(-74.0 \text { to } \\
-17.9)^{*}\end{array}$ & $\begin{array}{c}256 \cdot 3 \\
(145 \cdot 0 \text { to } \\
414 \cdot 8)\end{array}$ & $\begin{array}{l}109 \cdot 7 \\
(55 \cdot 8 \text { to } \\
197 \cdot 8)\end{array}$ & $\begin{array}{c}-58 \cdot 5(-75 \cdot 5 \text { to } \\
-22 \cdot 7)^{*}\end{array}$ \\
\hline Varicella and herpes zoster & $\begin{array}{c}463 \cdot 7 \\
(371 \cdot 2 \text { to } \\
580 \cdot 9)\end{array}$ & $\begin{array}{c}487.9 \\
(384 \cdot 7 \text { to } \\
622 \cdot 7)\end{array}$ & $5 \cdot 2(-12 \cdot 2$ to $26 \cdot 9)$ & $\begin{array}{l}7 \cdot 4(5 \cdot 9 \\
\text { to } 9 \cdot 2)\end{array}$ & $\begin{array}{l}7 \cdot 0(5 \cdot 5 \\
\text { to } 8 \cdot 9)\end{array}$ & $-6 \cdot 1(-20 \cdot 7$ to $12 \cdot 4)$ \\
\hline $\begin{array}{l}\text { Neglected tropical diseases and } \\
\text { malaria }\end{array}$ & $\begin{array}{c}121587 \cdot 6 \\
(108 \\
043 \cdot 8 \text { to } \\
136 \\
597 \cdot 5)\end{array}$ & $\begin{array}{c}90676 \cdot 8 \\
(75748 \cdot 9 \\
\text { to } 107 \\
737 \cdot 6)\end{array}$ & $\begin{array}{c}-25 \cdot 8(-34 \cdot 3 \text { to } \\
-14 \cdot 6)^{*}\end{array}$ & $\begin{array}{c}1798 \cdot 1 \\
(1596 \cdot 1 \\
\text { to } \\
2023 \cdot 0)\end{array}$ & $\begin{array}{c}1248 \cdot 4 \\
(1043 \cdot 0 \\
\text { to } \\
1483 \cdot 2)\end{array}$ & $\begin{array}{c}-30 \cdot 9(-38 \cdot 7 \text { to } \\
-20 \cdot 6)^{*}\end{array}$ \\
\hline Malaria & $\begin{array}{c}94497 \cdot 4 \\
\text { (83 } 484 \cdot 0 \\
\text { to } 106 \\
879 \cdot 0)\end{array}$ & $\begin{array}{c}65493 \cdot 1 \\
(53064 \cdot 9 \\
\text { to } 79 \\
960 \cdot 7)\end{array}$ & $\begin{array}{c}-31 \cdot 3(-41.6 \text { to } \\
-16.9)^{*}\end{array}$ & $\begin{array}{c}1385 \cdot 1 \\
(1224 \cdot 9 \\
\text { to } \\
1565 \cdot 9)\end{array}$ & $\begin{array}{c}897.6 \\
(728 \cdot 1 \text { to } \\
1094.8)\end{array}$ & $\begin{array}{c}-35 \cdot 7(-45 \cdot 3 \text { to } \\
-22 \cdot 3)^{*}\end{array}$ \\
\hline Chagas disease & $\begin{array}{l}326 \cdot 6 \\
(172 \cdot 5 \text { to } \\
850 \cdot 4)\end{array}$ & $\begin{array}{l}338 \cdot 5 \\
(183 \cdot 8 \text { to } \\
846 \cdot 4)\end{array}$ & $4 \cdot 6(-18 \cdot 4$ to $27 \cdot 8)$ & $\begin{array}{l}5 \cdot 9(3 \cdot 1 \\
\text { to } 15 \cdot 9)\end{array}$ & $\begin{array}{l}5 \cdot 2(2 \cdot 8 \\
\text { to } 12 \cdot 8)\end{array}$ & $-12 \cdot 3(-32.6$ to $7 \cdot 4)$ \\
\hline
\end{tabular}




\begin{tabular}{|c|c|c|c|c|c|c|}
\hline & \multicolumn{3}{|c|}{ All ages DALYs (thousands) ${ }^{*}$} & \multicolumn{3}{|c|}{$\underline{\text { Age-standardised DALYs (per } 100000)^{*}}$} \\
\hline & 2005 & 2013 & Percentage change & 2005 & 2013 & Percentage change \\
\hline Leishmaniasis & $\begin{array}{c}3939 \cdot 2 \\
(3292 \cdot 5 \text { to } \\
4619 \cdot 1)\end{array}$ & $\begin{array}{c}4283 \cdot 1 \\
(3527 \cdot 8 \text { to } \\
5090 \cdot 9)\end{array}$ & $8 \cdot 8(-6 \cdot 4$ to $25 \cdot 3)$ & $\begin{array}{c}57 \cdot 3 \\
(47.9 \text { to } \\
67 \cdot 1)\end{array}$ & $\begin{array}{c}58 \cdot 6 \\
(48 \cdot 2 \text { to } \\
69 \cdot 7)\end{array}$ & $2.5(-11.7$ to 18.0$)$ \\
\hline Visceral & $\begin{array}{c}3908.5 \\
(3272.6 \text { to } \\
4591.9)\end{array}$ & $\begin{array}{c}4241 \cdot 5 \\
(3488 \cdot 2 \text { to } \\
5044 \cdot 7)\end{array}$ & $8.7(-6.7$ to $25 \cdot 2)$ & $\begin{array}{c}56 \cdot 8 \\
(47 \cdot 5 \text { to } \\
66 \cdot 7)\end{array}$ & $\begin{array}{c}58 \cdot 0 \\
(47 \cdot 7 \text { to } \\
69 \cdot 0)\end{array}$ & $2 \cdot 4(-12 \cdot 0$ to $18 \cdot 0)$ \\
\hline Cutaneous and mucocutaneous & $\begin{array}{c}30 \cdot 6(14 \cdot 0 \\
\text { to } 58 \cdot 4)\end{array}$ & $\begin{array}{l}41 \cdot 7(19 \cdot 0 \\
\text { to } 80 \cdot 1)\end{array}$ & $35.9(23.7 \text { to } 49.0)^{*}$ & $\begin{array}{c}0.5(0.2 \\
\text { to } 0.9)\end{array}$ & $\begin{array}{l}0.6(0 \cdot 3 \\
\text { to } 1 \cdot 1)\end{array}$ & $23.0(12.3 \text { to } 34.8)^{*}$ \\
\hline African trypanosomiasis & $\begin{array}{c}854 \cdot 4 \\
(454 \cdot 4 \text { to } \\
1366 \cdot 7)\end{array}$ & $\begin{array}{c}390 \cdot 1 \\
(211 \cdot 4 \text { to } \\
615 \cdot 3)\end{array}$ & $\begin{array}{c}-54 \cdot 3(-58 \cdot 7 \text { to } \\
-49 \cdot 1)^{*}\end{array}$ & $\begin{array}{l}12 \cdot 6(6 \cdot 7 \\
\text { to } 20 \cdot 2)\end{array}$ & $\begin{array}{l}5 \cdot 3(2 \cdot 9 \\
\text { to } 8 \cdot 3)\end{array}$ & $\begin{array}{c}-58 \cdot 2(-62 \cdot 2 \text { to } \\
-53 \cdot 5)^{*}\end{array}$ \\
\hline Schistosomiasis & $\begin{array}{c}3511 \cdot 3 \\
(1999 \cdot 8 \text { to } \\
6207 \cdot 9)\end{array}$ & $\begin{array}{c}3062 \cdot 8 \\
(1690 \cdot 1 \text { to } \\
5662 \cdot 0)\end{array}$ & $\begin{array}{c}-13.9(-18.5 \text { to } \\
-1.4)^{*}\end{array}$ & $\begin{array}{c}52 \cdot 3 \\
(29 \cdot 9 \text { to } \\
92 \cdot 1)\end{array}$ & $\begin{array}{c}42 \cdot 1 \\
(23 \cdot 3 \text { to } \\
77 \cdot 8)\end{array}$ & $\begin{array}{c}-20 \cdot 5(-24 \cdot 7 \text { to } \\
-8 \cdot 8)^{*}\end{array}$ \\
\hline Cysticercosis & $\begin{array}{l}409 \cdot 7 \\
(291 \cdot 1 \text { to } \\
530 \cdot 4)\end{array}$ & $\begin{array}{c}341 \cdot 2 \\
(244 \cdot 4 \text { to } \\
442 \cdot 0)\end{array}$ & $-16 \cdot 4(-31 \cdot 7$ to $1 \cdot 3)$ & $\begin{array}{l}6 \cdot 4(4 \cdot 6 \\
\text { to } 8 \cdot 3)\end{array}$ & $\begin{array}{l}4.7(3.4 \\
\text { to } 6 \cdot 1)\end{array}$ & $\begin{array}{c}-26 \cdot 0(-39 \cdot 2 \text { to } \\
-11 \cdot 0)^{*}\end{array}$ \\
\hline Cystic echinococcosis & $\begin{array}{l}211 \cdot 5 \\
(185 \cdot 0 \text { to } \\
243 \cdot 3)\end{array}$ & $\begin{array}{l}181.7 \\
(155 \cdot 7 \text { to } \\
211.7)\end{array}$ & $\begin{array}{c}-14 \cdot 1(-17 \cdot 3 \text { to } \\
-11 \cdot 1)^{*}\end{array}$ & $\begin{array}{l}3 \cdot 3(2 \cdot 9 \\
\text { to } 3 \cdot 8)\end{array}$ & $\begin{array}{l}2 \cdot 6(2 \cdot 2 \\
\text { to } 3 \cdot 0)\end{array}$ & $\begin{array}{c}-22 \cdot 3(-25 \cdot 0 \text { to } \\
-19 \cdot 7)^{*}\end{array}$ \\
\hline Lymphatic filariasis & $\begin{array}{c}2406 \cdot 4 \\
(1241 \cdot 2 \text { to } \\
4094 \cdot 3)\end{array}$ & $\begin{array}{c}2022 \cdot 1 \\
(1096 \cdot 3 \text { to } \\
3294 \cdot 4)\end{array}$ & $\begin{array}{c}-14 \cdot 3(-31 \cdot 4 \text { to } \\
-5 \cdot 3)^{*}\end{array}$ & $\begin{array}{c}39 \cdot 6 \\
(20 \cdot 4 \text { to } \\
67 \cdot 1)\end{array}$ & $\begin{array}{l}28 \cdot 9 \\
(15 \cdot 7 \text { to } \\
47 \cdot 1)\end{array}$ & $\begin{array}{c}-25 \cdot 7(-40 \cdot 1 \text { to } \\
-18 \cdot 0)^{*}\end{array}$ \\
\hline Onchocerciasis & $\begin{array}{c}1445 \cdot 3 \\
(792 \cdot 4 \text { to } \\
2241 \cdot 9)\end{array}$ & $\begin{array}{c}1179 \cdot 8 \\
(556 \cdot 6 \text { to } \\
1992 \cdot 7)\end{array}$ & $\begin{array}{c}-19 \cdot 4(-33 \cdot 0 \text { to } \\
-5 \cdot 0)^{*}\end{array}$ & $\begin{array}{l}22 \cdot 6 \\
(12 \cdot 6 \text { to } \\
34 \cdot 4)\end{array}$ & $\begin{array}{l}16 \cdot 6(7.9 \\
\text { to } 27 \cdot 6)\end{array}$ & $\begin{array}{c}-27 \cdot 5(-40 \cdot 0 \text { to } \\
-14 \cdot 4)^{*}\end{array}$ \\
\hline Trachoma & $\begin{array}{c}208.9 \\
(141 \cdot 3 \text { to } \\
286 \cdot 9)\end{array}$ & $\begin{array}{l}171 \cdot 2 \\
(115 \cdot 3 \text { to } \\
241 \cdot 7)\end{array}$ & $\begin{array}{c}-18 \cdot 1(-27 \cdot 5 \text { to } \\
-8 \cdot 4)^{*}\end{array}$ & $\begin{array}{l}4.2(2 \cdot 9 \\
\text { to } 5 \cdot 8)\end{array}$ & $\begin{array}{l}2.8(1.9 \\
\text { to } 4.0)\end{array}$ & $\begin{array}{c}-33 \cdot 4(-41 \cdot 0 \text { to } \\
-25 \cdot 6)^{*}\end{array}$ \\
\hline Dengue & $\begin{array}{l}957.9 \\
(627.9 \text { to } \\
1395 \cdot 8)\end{array}$ & $\begin{array}{c}1142 \cdot 7 \\
(727 \cdot 6 \text { to } \\
1978 \cdot 2)\end{array}$ & $17 \cdot 0(-7 \cdot 9$ to $53 \cdot 1)$ & $\begin{array}{l}14 \cdot 1(9 \cdot 3 \\
\text { to } 20 \cdot 6)\end{array}$ & $\begin{array}{l}15 \cdot 8 \\
(10 \cdot 1 \text { to } \\
27 \cdot 4)\end{array}$ & $9 \cdot 8(-13 \cdot 3$ to $43 \cdot 0)$ \\
\hline Yellow fever & $\begin{array}{c}30 \cdot 2(25 \cdot 1 \\
\text { to } 36 \cdot 8)\end{array}$ & $\begin{array}{c}30 \cdot 7(25 \cdot 3 \\
\text { to } 37 \cdot 1)\end{array}$ & $1 \cdot 8(-18 \cdot 2$ to $25 \cdot 3)$ & $\begin{array}{l}0.4(0.4 \\
\text { to } 0.5)\end{array}$ & $\begin{array}{l}0.4(0.3 \\
\text { to } 0.5)\end{array}$ & $-4 \cdot 2(-22 \cdot 9$ to $17 \cdot 5)$ \\
\hline Rabies & $\begin{array}{c}1449 \cdot 7 \\
(1124 \cdot 4 \text { to } \\
1833 \cdot 1)\end{array}$ & $\begin{array}{c}1242 \cdot 9 \\
(914 \cdot 6 \text { to } \\
1526 \cdot 7)\end{array}$ & $-14 \cdot 6(-27 \cdot 6$ to $0 \cdot 9)$ & $\begin{array}{c}21 \cdot 8 \\
(16 \cdot 9 \text { to } \\
27 \cdot 5)\end{array}$ & $\begin{array}{l}17 \cdot 3 \\
(12 \cdot 7 \text { to } \\
21 \cdot 2)\end{array}$ & $\begin{array}{c}-20 \cdot 9(-32 \cdot 9 \text { to } \\
-6 \cdot 5)^{*}\end{array}$ \\
\hline Intestinal nematode infections & $\begin{array}{c}4641 \cdot 3 \\
(2899 \cdot 4 \text { to } \\
7110 \cdot 5)\end{array}$ & $\begin{array}{c}4029 \cdot 4 \\
(2516 \cdot 8 \text { to } \\
6137 \cdot 0)\end{array}$ & $\begin{array}{c}-13 \cdot 1(-18 \cdot 3 \text { to } \\
-7 \cdot 8)^{*}\end{array}$ & $\begin{array}{c}69 \cdot 4 \\
(43 \cdot 3 \text { to } \\
106 \cdot 4)\end{array}$ & $\begin{array}{c}55 \cdot 7 \\
(34 \cdot 8 \text { to } \\
84 \cdot 9)\end{array}$ & $\begin{array}{c}-19.6(-24 \cdot 4 \text { to } \\
-14 \cdot 7)^{*}\end{array}$ \\
\hline Ascariasis & $\begin{array}{c}1796 \cdot 2 \\
(1150 \cdot 3 \text { to } \\
2720 \cdot 3)\end{array}$ & $\begin{array}{c}1271 \cdot 7 \\
(843 \cdot 1 \text { to } \\
1916 \cdot 7)\end{array}$ & $\begin{array}{c}-29 \cdot 0(-35 \cdot 9 \text { to } \\
-21 \cdot 3)^{*}\end{array}$ & $\begin{array}{l}26 \cdot 8 \\
(17 \cdot 2 \text { to } \\
40 \cdot 7)\end{array}$ & $\begin{array}{l}17 \cdot 6 \\
(11 \cdot 6 \text { to } \\
26 \cdot 5)\end{array}$ & $\begin{array}{c}-34 \cdot 3(-40 \cdot 7 \text { to } \\
-27 \cdot 2)^{*}\end{array}$ \\
\hline Trichuriasis & $\begin{array}{l}652 \cdot 0 \\
(357 \cdot 4 \text { to } \\
1063 \cdot 6)\end{array}$ & $\begin{array}{l}576 \cdot 0 \\
(310 \cdot 1 \text { to } \\
972 \cdot 6)\end{array}$ & $-12 \cdot 3(-26 \cdot 3$ to $8 \cdot 8)$ & $\begin{array}{l}9 \cdot 8(5 \cdot 4 \\
\text { to } 16 \cdot 0)\end{array}$ & $\begin{array}{l}8 \cdot 0(4 \cdot 3 \\
\text { to } 13 \cdot 5)\end{array}$ & $-19 \cdot 1(-32 \cdot 1$ to $0 \cdot 4)$ \\
\hline Hookworm disease & $\begin{array}{l}2193 \cdot 2 \\
(1335 \cdot 6 \text { to } \\
3401 \cdot 2)\end{array}$ & $\begin{array}{c}2181 \cdot 7 \\
(1338 \cdot 6 \text { to } \\
3354 \cdot 5)\end{array}$ & $-0.5(-6.9$ to $6 \cdot 6)$ & $\begin{array}{c}32 \cdot 8 \\
(20 \cdot 0 \text { to } \\
50 \cdot 8)\end{array}$ & $\begin{array}{l}30 \cdot 2 \\
(18 \cdot 5 \text { to } \\
46 \cdot 4)\end{array}$ & $-7.8(-13.9 \text { to }-1.3)^{*}$ \\
\hline Food-borne trematodiases & $\begin{array}{c}3161.5 \\
(1039.8 \text { to } \\
6574.9)\end{array}$ & $\begin{array}{c}3634 \cdot 8 \\
(1160 \cdot 2 \text { to } \\
7692 \cdot 4)\end{array}$ & $14.6(8 \cdot 6 \text { to } 23 \cdot 2)^{*}$ & $\begin{array}{c}51 \cdot 3 \\
(16 \cdot 8 \text { to } \\
106 \cdot 9)\end{array}$ & $\begin{array}{c}51 \cdot 3 \\
(16 \cdot 3 \text { to } \\
108 \cdot 6)\end{array}$ & $-0 \cdot 3(-5 \cdot 4$ to $6 \cdot 5)$ \\
\hline Other neglected tropical diseases & $\begin{array}{c}3536 \cdot 4 \\
(2652 \cdot 7 \text { to } \\
4638 \cdot 1)\end{array}$ & $\begin{array}{c}3132 \cdot 7 \\
(2328 \cdot 1 \text { to } \\
4208 \cdot 7)\end{array}$ & $\begin{array}{c}-11 \cdot 8(-18 \cdot 0 \text { to } \\
-3 \cdot 1)^{*}\end{array}$ & $\begin{array}{c}51 \cdot 8 \\
(38.9 \text { to } \\
67 \cdot 8)\end{array}$ & $\begin{array}{c}43 \cdot 5 \\
(32 \cdot 3 \text { to } \\
58 \cdot 4)\end{array}$ & $\begin{array}{c}-16 \cdot 3(-22 \cdot 2 \text { to } \\
-8 \cdot 3)^{*}\end{array}$ \\
\hline
\end{tabular}




\begin{tabular}{|c|c|c|c|c|c|c|}
\hline & \multicolumn{3}{|c|}{ All ages DALYs (thousands) ${ }^{*}$} & \multicolumn{3}{|c|}{$\underline{\text { Age-standardised DALYs (per } 100000 \text { ) }}$} \\
\hline & 2005 & 2013 & Percentage change & 2005 & 2013 & Percentage change \\
\hline Maternal disorders & $\begin{array}{c}21717 \cdot 2 \\
(19935 \cdot 4 \\
\text { to } 23 \\
449 \cdot 9)\end{array}$ & $\begin{array}{c}18027 \cdot 8 \\
(16051 \cdot 8 \\
\text { to } 19 \\
989 \cdot 5)\end{array}$ & $\begin{array}{c}-17 \cdot 0(-25 \cdot 6 \text { to } \\
-7 \cdot 9)^{*}\end{array}$ & $\begin{array}{c}312 \cdot 7 \\
(287 \cdot 2 \text { to } \\
337 \cdot 5)\end{array}$ & $\begin{array}{c}239 \cdot 2 \\
(213 \cdot 3 \text { to } \\
264 \cdot 9)\end{array}$ & $\begin{array}{c}-23 \cdot 5(-31 \cdot 4 \text { to } \\
-15 \cdot 3)^{*}\end{array}$ \\
\hline Maternal haemorrhage & $\begin{array}{c}3551 \cdot 9 \\
(3154 \cdot 8 \text { to } \\
3980 \cdot 3)\end{array}$ & $\begin{array}{c}2561 \cdot 7 \\
(2219 \cdot 9 \text { to } \\
2926 \cdot 6)\end{array}$ & $\begin{array}{c}-28 \cdot 2(-38.0 \text { to } \\
-16 \cdot 3)^{*}\end{array}$ & $\begin{array}{c}51 \cdot 3 \\
(45 \cdot 6 \text { to } \\
57 \cdot 5)\end{array}$ & $\begin{array}{c}34 \cdot 0 \\
(29 \cdot 5 \text { to } \\
38 \cdot 9)\end{array}$ & $\begin{array}{c}-34 \cdot 0(-42 \cdot 7 \text { to } \\
-23 \cdot 0)^{*}\end{array}$ \\
\hline $\begin{array}{l}\text { Maternal sepsis and other } \\
\text { maternal infections }\end{array}$ & $\begin{array}{c}1781 \cdot 7 \\
(1580 \cdot 7 \text { to } \\
2007 \cdot 9)\end{array}$ & $\begin{array}{c}1369 \cdot 6 \\
(1156 \cdot 9 \text { to } \\
1624 \cdot 0)\end{array}$ & $\begin{array}{c}-23 \cdot 5(-36 \cdot 2 \text { to } \\
-7 \cdot 9)^{*}\end{array}$ & $\begin{array}{l}25 \cdot 6 \\
(22 \cdot 7 \text { to } \\
28 \cdot 8)\end{array}$ & $\begin{array}{l}18 \cdot 2 \\
(15 \cdot 3 \text { to } \\
21 \cdot 5)\end{array}$ & $\begin{array}{c}-29 \cdot 4(-41.0 \text { to } \\
-15 \cdot 1)^{*}\end{array}$ \\
\hline Maternal hypertensive disorders & $\begin{array}{c}2281 \cdot 0 \\
(2038 \cdot 0 \text { to } \\
2547 \cdot 4)\end{array}$ & $\begin{array}{c}1753 \cdot 2 \\
(1523 \cdot 0 \text { to } \\
1996 \cdot 7)\end{array}$ & $\begin{array}{c}-23 \cdot 4(-32 \cdot 7 \text { to } \\
-10 \cdot 8)^{*}\end{array}$ & $\begin{array}{c}32 \cdot 6 \\
(29 \cdot 2 \text { to } \\
36 \cdot 4)\end{array}$ & $\begin{array}{l}23 \cdot 2 \\
(20 \cdot 2 \text { to } \\
26 \cdot 4)\end{array}$ & $\begin{array}{c}-29 \cdot 1(-37 \cdot 5 \text { to } \\
-17 \cdot 3)^{*}\end{array}$ \\
\hline Obstructed labour & $\begin{array}{c}2312 \cdot 0 \\
(1963 \cdot 9 \text { to } \\
2679 \cdot 8)\end{array}$ & $\begin{array}{c}2023 \cdot 4 \\
(1686 \cdot 8 \text { to } \\
2414 \cdot 0)\end{array}$ & $\begin{array}{c}-12 \cdot 5(-20 \cdot 5 \text { to } \\
-3 \cdot 8)^{*}\end{array}$ & $\begin{array}{c}33 \cdot 6 \\
(28 \cdot 5 \text { to } \\
39 \cdot 0)\end{array}$ & $\begin{array}{l}27 \cdot 1 \\
(22 \cdot 5 \text { to } \\
32 \cdot 3)\end{array}$ & $\begin{array}{c}-19 \cdot 5(-26.9 \text { to } \\
-11 \cdot 6)^{*}\end{array}$ \\
\hline Complications of abortion & $\begin{array}{c}2886 \cdot 2 \\
(2603 \cdot 8 \text { to } \\
3192 \cdot 2)\end{array}$ & $\begin{array}{l}2476 \cdot 5 \\
(2169 \cdot 9 \text { to } \\
2841 \cdot 6)\end{array}$ & $\begin{array}{c}-14 \cdot 6(-24 \cdot 6 \text { to } \\
-0 \cdot 5)^{*}\end{array}$ & $\begin{array}{c}41 \cdot 6 \\
(37 \cdot 6 \text { to } \\
46 \cdot 0)\end{array}$ & $\begin{array}{c}32 \cdot 8 \\
(28 \cdot 7 \text { to } \\
37 \cdot 6)\end{array}$ & $\begin{array}{c}-21 \cdot 6(-30 \cdot 8 \text { to } \\
-8 \cdot 8)^{*}\end{array}$ \\
\hline Indirect maternal deaths & $\begin{array}{c}2391 \cdot 2 \\
(2110 \cdot 7 \text { to } \\
2718 \cdot 2)\end{array}$ & $\begin{array}{c}1790 \cdot 8 \\
(1534 \cdot 9 \text { to } \\
2074 \cdot 7)\end{array}$ & $\begin{array}{c}-25 \cdot 3(-37 \cdot 7 \text { to } \\
-9 \cdot 9)^{*}\end{array}$ & $\begin{array}{c}34 \cdot 3 \\
(30 \cdot 3 \text { to } \\
38 \cdot 9)\end{array}$ & $\begin{array}{l}23 \cdot 7 \\
(20 \cdot 3 \text { to } \\
27 \cdot 4)\end{array}$ & $\begin{array}{c}-31 \cdot 1(-42 \cdot 4 \text { to } \\
-17 \cdot 2)^{*}\end{array}$ \\
\hline Late maternal deaths & $\begin{array}{c}2525 \cdot 7 \\
(2151 \cdot 7 \text { to } \\
2947 \cdot 8)\end{array}$ & $\begin{array}{c}2481 \cdot 8 \\
(2030 \cdot 4 \text { to } \\
2992 \cdot 2)\end{array}$ & $-1 \cdot 3(-23 \cdot 0$ to $23 \cdot 2)$ & $\begin{array}{c}36 \cdot 2 \\
(30 \cdot 8 \text { to } \\
42 \cdot 2)\end{array}$ & $\begin{array}{l}32.9 \\
(27 \cdot 0 \text { to } \\
39 \cdot 7)\end{array}$ & $-8 \cdot 6(-28 \cdot 6$ to $13 \cdot 7)$ \\
\hline $\begin{array}{l}\text { Maternal deaths aggravated by } \\
\text { HIV/AIDS }\end{array}$ & $\begin{array}{l}184 \cdot 7 \\
(113 \cdot 8 \text { to } \\
248 \cdot 6)\end{array}$ & $\begin{array}{c}117.2 \\
(72 \cdot 6 \text { to } \\
162 \cdot 7)\end{array}$ & $\begin{array}{c}-36 \cdot 6(-43.9 \text { to } \\
-27 \cdot 7)^{*}\end{array}$ & $\begin{array}{l}2.7(1.7 \\
\text { to } 3 \cdot 6)\end{array}$ & $\begin{array}{l}1.5(1 \cdot 0 \\
\text { to } 2 \cdot 1)\end{array}$ & $\begin{array}{c}-42 \cdot 5(-49 \cdot 2 \text { to } \\
-34 \cdot 3)^{*}\end{array}$ \\
\hline Other maternal disorders & $\begin{array}{c}3761 \cdot 6 \\
(3379 \cdot 7 \text { to } \\
4197 \cdot 0)\end{array}$ & $\begin{array}{c}3420 \cdot 6 \\
(2936 \cdot 5 \text { to } \\
3954 \cdot 0)\end{array}$ & $-9 \cdot 4(-21 \cdot 3$ to $7 \cdot 9)$ & $\begin{array}{c}54 \cdot 2 \\
(48 \cdot 7 \text { to } \\
60 \cdot 4)\end{array}$ & $\begin{array}{l}45 \cdot 4 \\
(39 \cdot 0 \text { to } \\
52 \cdot 4)\end{array}$ & $\begin{array}{c}-16 \cdot 6(-27 \cdot 5 \text { to } \\
-0 \cdot 5)^{*}\end{array}$ \\
\hline Neonatal disorders & $\begin{array}{c}221687 \cdot 5 \\
(213 \\
295 \cdot 0 \text { to } \\
230 \\
283 \cdot 3)\end{array}$ & $\begin{array}{c}189601 \cdot 0 \\
(179 \\
024 \cdot 1 \text { to } \\
200 \\
044 \cdot 0)\end{array}$ & $\begin{array}{c}-14 \cdot 5(-18 \cdot 1 \text { to } \\
-10 \cdot 7)^{*}\end{array}$ & $\begin{array}{c}\mathbf{3 1 5 5 \cdot 7} \\
(\mathbf{3 0 3 5} \cdot 7 \\
\text { to } \\
\mathbf{3 2 7 8} \cdot 5)\end{array}$ & $\begin{array}{c}2560 \cdot 0 \\
(2416 \cdot 8 \\
\text { to } \\
2700 \cdot 5)\end{array}$ & $\begin{array}{c}-18 \cdot 9(-22 \cdot 3 \text { to } \\
-15 \cdot 3)^{*}\end{array}$ \\
\hline Preterm birth complications & $\begin{array}{c}88971 \cdot 6 \\
(75761.9 \\
\text { to } 108 \\
464 \cdot 4)\end{array}$ & $\begin{array}{c}70843 \cdot 1 \\
(57523 \cdot 4 \\
\text { to } 85 \\
348 \cdot 6)\end{array}$ & $\begin{array}{c}-20 \cdot 3(-28 \cdot 6 \text { to } \\
-12 \cdot 2)^{*}\end{array}$ & $\begin{array}{c}1267 \cdot 2 \\
(1079 \cdot 1 \\
\text { to } \\
1543 \cdot 9)\end{array}$ & $\begin{array}{c}957 \cdot 0 \\
(777 \cdot 2 \text { to } \\
1152 \cdot 8)\end{array}$ & $\begin{array}{c}-24.4(-32.3 \text { to } \\
-16 \cdot 7)^{*}\end{array}$ \\
\hline $\begin{array}{l}\text { Neonatal encephalopathy (birth } \\
\text { asphyxia and trauma) }\end{array}$ & $\begin{array}{c}66760 \cdot 9 \\
(53355 \cdot 4 \\
\text { to } 77 \\
432 \cdot 1)\end{array}$ & $\begin{array}{c}58012 \cdot 7 \\
(46947 \cdot 7 \\
\text { to } 68 \\
198 \cdot 2)\end{array}$ & $\begin{array}{c}-13 \cdot 2(-22 \cdot 7 \text { to } \\
-1 \cdot 8)^{*}\end{array}$ & $\begin{array}{c}948.9 \\
(758 \cdot 6 \text { to } \\
1100 \cdot 2)\end{array}$ & $\begin{array}{l}782.6 \\
(633.4 \text { to } \\
919.7)\end{array}$ & $\begin{array}{c}-17 \cdot 6(-26 \cdot 6 \text { to } \\
-6 \cdot 8)^{*}\end{array}$ \\
\hline $\begin{array}{l}\text { Sepsis and other neonatal } \\
\text { infections }\end{array}$ & $\begin{array}{c}32000 \cdot 2 \\
(19472 \cdot 8 \\
\text { to } 44 \\
418 \cdot 3)\end{array}$ & $\begin{array}{c}31631.8 \\
(20147.7 \\
\text { to } 44 \\
128.5)\end{array}$ & $-1 \cdot 0(-16 \cdot 8$ to $19 \cdot 3)$ & $\begin{array}{l}455 \cdot 0 \\
(277 \cdot 0 \text { to } \\
631 \cdot 4)\end{array}$ & $\begin{array}{l}426 \cdot 7 \\
(271 \cdot 9 \text { to } \\
595 \cdot 3)\end{array}$ & $-6 \cdot 1(-21 \cdot 1$ to $13 \cdot 2)$ \\
\hline $\begin{array}{l}\text { Haemolytic disease and other } \\
\text { neonatal jaundice }\end{array}$ & $\begin{array}{c}3639 \cdot 6 \\
(2720 \cdot 1 \text { to } \\
4907 \cdot 6)\end{array}$ & $\begin{array}{c}3299 \cdot 4 \\
(2496 \cdot 2 \text { to } \\
4382 \cdot 9)\end{array}$ & $-9.0(-29 \cdot 7$ to 14.7$)$ & $\begin{array}{c}52 \cdot 5 \\
(39 \cdot 3 \text { to } \\
70 \cdot 7)\end{array}$ & $\begin{array}{c}44.9 \\
(33.9 \text { to } \\
59 \cdot 6)\end{array}$ & $-14 \cdot 2(-33 \cdot 6$ to $8 \cdot 0)$ \\
\hline Other neonatal disorders & $\begin{array}{c}30315 \cdot 2 \\
(24647 \cdot 8 \\
\text { to } 38 \\
101 \cdot 8)\end{array}$ & $\begin{array}{c}25814 \cdot 0 \\
(20483.9 \\
\text { to } 32 \\
840 \cdot 8)\end{array}$ & $-14.7(-27 \cdot 3$ to 0.7$)$ & $\begin{array}{c}432 \cdot 1 \\
(351 \cdot 0 \text { to } \\
542 \cdot 8)\end{array}$ & $\begin{array}{c}348 \cdot 8 \\
(276 \cdot 7 \text { to } \\
443 \cdot 7)\end{array}$ & $\begin{array}{c}-19 \cdot 1(-31 \cdot 1 \text { to } \\
-4 \cdot 6)^{*}\end{array}$ \\
\hline
\end{tabular}




\begin{tabular}{|c|c|c|c|c|c|c|}
\hline & \multicolumn{3}{|c|}{ All ages DALYs (thousands) ${ }^{*}$} & \multicolumn{3}{|c|}{$\underline{\text { Age-standardised DALYs (per } 100000)^{*}}$} \\
\hline & 2005 & 2013 & Percentage change & 2005 & 2013 & Percentage change \\
\hline Nutritional deficiencies & $\begin{array}{c}79695 \cdot 9 \\
(63911 \cdot 4 \\
\text { to } 99 \\
518 \cdot 1)\end{array}$ & $\begin{array}{c}74834 \cdot 4 \\
(59402 \cdot 0 \\
\text { to } 94 \\
084 \cdot 1)\end{array}$ & $-6 \cdot 2(-10 \cdot 9 \text { to }-1 \cdot 4)^{*}$ & $\begin{array}{c}1192 \cdot 4 \\
(958 \cdot 9 \text { to } \\
1486 \cdot 1)\end{array}$ & $\begin{array}{c}1040 \cdot 7 \\
(828 \cdot 2 \text { to } \\
1306 \cdot 8)\end{array}$ & $\begin{array}{c}-12 \cdot 8(-17 \cdot 1 \text { to } \\
-8 \cdot 5)^{*}\end{array}$ \\
\hline Protein-energy malnutrition & $\begin{array}{c}29772 \cdot 1 \\
(23294 \cdot 2 \\
\text { to } 35 \\
802 \cdot 7)\end{array}$ & $\begin{array}{c}27709 \cdot 9 \\
(21411 \cdot 5 \\
\text { to } 33 \\
507 \cdot 3)\end{array}$ & $-7 \cdot 1(-17 \cdot 5$ to $4 \cdot 5)$ & $\begin{array}{c}447 \cdot 4 \\
(348 \cdot 0 \text { to } \\
536 \cdot 2)\end{array}$ & $\begin{array}{c}386 \cdot 1 \\
(298 \cdot 4 \text { to } \\
465 \cdot 7)\end{array}$ & $\begin{array}{c}-13 \cdot 7(-23 \cdot 1 \text { to } \\
-3 \cdot 6)^{*}\end{array}$ \\
\hline Iodine deficiency & $\begin{array}{c}2155 \cdot 1 \\
(1364 \cdot 0 \text { to } \\
3259 \cdot 2)\end{array}$ & $\begin{array}{l}2189 \cdot 6 \\
(1406 \cdot 6 \text { to } \\
3401 \cdot 4)\end{array}$ & $1.5(-5.9$ to $9 \cdot 8)$ & $\begin{array}{c}32 \cdot 6 \\
(20 \cdot 7 \text { to } \\
49 \cdot 4)\end{array}$ & $\begin{array}{c}30 \cdot 1 \\
(19 \cdot 3 \text { to } \\
46 \cdot 7)\end{array}$ & $-7.9(-14.7 \text { to }-0.3)^{*}$ \\
\hline Vitamin A deficiency & $\begin{array}{c}177 \cdot 2 \\
(113 \cdot 8 \text { to } \\
261 \cdot 1)\end{array}$ & $\begin{array}{c}153 \cdot 7 \\
(99 \cdot 0 \text { to } \\
224 \cdot 9)\end{array}$ & $\begin{array}{c}-13 \cdot 2(-19 \cdot 5 \text { to } \\
-6 \cdot 8)^{*}\end{array}$ & $\begin{array}{l}2.6(1.7 \\
\text { to } 3 \cdot 9)\end{array}$ & $\begin{array}{l}2 \cdot 1(1 \cdot 4 \\
\text { to } 3 \cdot 1)\end{array}$ & $\begin{array}{c}-19 \cdot 1(-24 \cdot 8 \text { to } \\
-13 \cdot 2)\end{array}$ \\
\hline Iron-deficiency anaemia & $\begin{array}{c}46359 \cdot 5 \\
(33059 \cdot 4 \\
\text { to } 64 \\
257 \cdot 3)\end{array}$ & $\begin{array}{c}43747 \cdot 6 \\
(30848 \cdot 7 \\
\text { to } 61 \\
398 \cdot 4)\end{array}$ & $-5 \cdot 6(-8 \cdot 2$ to $-3 \cdot 6) *$ & $\begin{array}{c}690 \cdot 1 \\
(494 \cdot 3 \text { to } \\
954 \cdot 8)\end{array}$ & $\begin{array}{c}607 \cdot 6 \\
(428 \cdot 9 \text { to } \\
852 \cdot 3)\end{array}$ & $\begin{array}{c}-12 \cdot 0(-14 \cdot 4 \text { to } \\
-10 \cdot 0)\end{array}$ \\
\hline Other nutritional deficiencies & $\begin{array}{l}1232 \cdot 0 \\
(847 \cdot 8 \text { to } \\
2025 \cdot 2)\end{array}$ & $\begin{array}{c}1033 \cdot 5 \\
(715 \cdot 8 \text { to } \\
1747 \cdot 2)\end{array}$ & $\begin{array}{c}-16 \cdot 1(-29 \cdot 0 \text { to } \\
-2 \cdot 5)^{*}\end{array}$ & $\begin{array}{l}19.6 \\
(13 \cdot 4 \text { to } \\
31 \cdot 8)\end{array}$ & $\begin{array}{l}14 \cdot 8 \\
\left(\begin{array}{c}10 \cdot 3 \text { to } \\
25 \cdot 1)\end{array}\right.\end{array}$ & $\begin{array}{c}-24 \cdot 3(-35 \cdot 1 \text { to } \\
-12 \cdot 3)^{*}\end{array}$ \\
\hline $\begin{array}{l}\text { Other communicable, maternal, } \\
\text { neonatal, and nutritional diseases }\end{array}$ & $\begin{array}{c}31457 \cdot 6 \\
(24584 \cdot 5 \\
\text { to } 39 \\
680 \cdot 1)\end{array}$ & $\begin{array}{c}27114 \cdot 0 \\
(21684 \cdot 1 \\
\text { to } 33 \\
977 \cdot 7)\end{array}$ & $\begin{array}{c}-13 \cdot 5(-23 \cdot 8 \text { to } \\
-3 \cdot 7)^{*}\end{array}$ & $\begin{array}{c}466 \cdot 5 \\
(367 \cdot 2 \text { to } \\
585 \cdot 0)\end{array}$ & $\begin{array}{c}373 \cdot 8 \\
(300 \cdot 0 \text { to } \\
466 \cdot 4)\end{array}$ & $\begin{array}{c}-19 \cdot 7(-29 \cdot 1 \text { to } \\
-10 \cdot 7)^{*}\end{array}$ \\
\hline $\begin{array}{l}\text { Sexually transmitted diseases } \\
\text { excluding HIV }\end{array}$ & $\begin{array}{l}15145 \cdot 4 \\
(9593 \cdot 7 \text { to } \\
22186 \cdot 1)\end{array}$ & $\begin{array}{c}12857 \cdot 2 \\
(8079 \cdot 7 \text { to } \\
19013 \cdot 3)\end{array}$ & $\begin{array}{c}-14.9(-30 \cdot 4 \text { to } \\
-0 \cdot 2)^{*}\end{array}$ & $\begin{array}{l}218 \cdot 6 \\
(139 \cdot 2 \text { to } \\
319 \cdot 6)\end{array}$ & $\begin{array}{l}174 \cdot 6 \\
(109 \cdot 8 \text { to } \\
258 \cdot 0)\end{array}$ & $\begin{array}{c}-19 \cdot 9(-34 \cdot 5 \text { to } \\
-6 \cdot 2)^{*}\end{array}$ \\
\hline Syphilis & $\begin{array}{c}13710 \cdot 1 \\
(8228 \cdot 6 \text { to } \\
20649 \cdot 7)\end{array}$ & $\begin{array}{c}11324 \cdot 5 \\
(6634 \cdot 9 \text { to } \\
17484 \cdot 8)\end{array}$ & $\begin{array}{c}-17 \cdot 1(-33 \cdot 7 \text { to } \\
-1 \cdot 2)^{*}\end{array}$ & $\begin{array}{c}197 \cdot 1 \\
(118 \cdot 6 \text { to } \\
296 \cdot 5)\end{array}$ & $\begin{array}{l}153 \cdot 8 \\
(90 \cdot 3 \text { to } \\
237 \cdot 3)\end{array}$ & $\begin{array}{c}-21 \cdot 8(-37 \cdot 3 \text { to } \\
-6 \cdot 7)^{*}\end{array}$ \\
\hline Chlamydial infection & $\begin{array}{c}645 \cdot 9 \\
(424 \cdot 5 \text { to } \\
990 \cdot 0)\end{array}$ & $\begin{array}{c}692 \cdot 4 \\
(454 \cdot 5 \text { to } \\
1065 \cdot 5)\end{array}$ & $7 \cdot 2(2 \cdot 2 \text { to } 12 \cdot 7)^{*}$ & $\begin{array}{l}9 \cdot 4(6 \cdot 2 \\
\text { to } 14 \cdot 4)\end{array}$ & $\begin{array}{l}9 \cdot 3(6 \cdot 1 \\
\text { to } 14 \cdot 2)\end{array}$ & $-1 \cdot 6(-6 \cdot 4$ to $3 \cdot 4)$ \\
\hline Gonococcal infection & $\begin{array}{c}293.9 \\
(219 \cdot 3 \text { to } \\
401 \cdot 1)\end{array}$ & $\begin{array}{c}313 \cdot 9 \\
(229 \cdot 4 \text { to } \\
438 \cdot 1)\end{array}$ & $6 \cdot 8(-3 \cdot 2$ to $16 \cdot 8)$ & $\begin{array}{l}4 \cdot 3(3 \cdot 3 \\
\text { to } 5 \cdot 8)\end{array}$ & $\begin{array}{l}4 \cdot 2(3 \cdot 1 \\
\text { to } 5 \cdot 9)\end{array}$ & $-2 \cdot 3(-11 \cdot 5$ to $7 \cdot 1)$ \\
\hline Trichomoniasis & $\begin{array}{l}105 \cdot 1 \\
(41 \cdot 3 \text { to } \\
221 \cdot 3)\end{array}$ & $\begin{array}{l}113 \cdot 9 \\
(45 \cdot 1 \text { to } \\
242 \cdot 9)\end{array}$ & $8 \cdot 2(-1 \cdot 8$ to $20 \cdot 0)$ & $\begin{array}{l}1.5(0 \cdot 6 \\
\text { to } 3 \cdot 2)\end{array}$ & $\begin{array}{l}1.5(0 \cdot 6 \\
\text { to } 3 \cdot 2)\end{array}$ & $-0 \cdot 8(-9 \cdot 9$ to $10 \cdot 0)$ \\
\hline Genital herpes & $\begin{array}{l}279.9 \\
(89 \cdot 6 \text { to } \\
671 \cdot 1)\end{array}$ & $\begin{array}{l}311 \cdot 6 \\
(98 \cdot 3 \text { to } \\
748 \cdot 5)\end{array}$ & $11.2(8.5 \text { to } 13 \cdot 6)^{*}$ & $\begin{array}{l}4.5(1.4 \\
\text { to } 10 \cdot 9)\end{array}$ & $\begin{array}{l}4.4(1.4 \\
\text { to } 10 \cdot 5)\end{array}$ & $-3 \cdot 0(-4.9 \text { to }-1 \cdot 0)^{*}$ \\
\hline $\begin{array}{l}\text { Other sexually transmitted } \\
\text { diseases }\end{array}$ & $\begin{array}{l}110 \cdot 5 \\
(93 \cdot 5 \text { to } \\
133 \cdot 7)\end{array}$ & $\begin{array}{l}101 \cdot 0 \\
(86 \cdot 1 \text { to } \\
121 \cdot 0)\end{array}$ & $-8 \cdot 8(-16 \cdot 9$ to $2 \cdot 4)$ & $\begin{array}{l}1.7(1.4 \\
\text { to } 2 \cdot 0)\end{array}$ & $\begin{array}{l}1.4(1.2 \\
\text { to } 1.7)\end{array}$ & $\begin{array}{c}-17 \cdot 7(-25 \cdot 2 \text { to } \\
-7 \cdot 6)^{*}\end{array}$ \\
\hline Hepatitis & $\begin{array}{c}7094 \cdot 0 \\
(6392 \cdot 5 \text { to } \\
8180 \cdot 0)\end{array}$ & $\begin{array}{c}6556 \cdot 8 \\
(5774 \cdot 7 \text { to } \\
8208 \cdot 0)\end{array}$ & $-8 \cdot 2(-17 \cdot 4$ to $5 \cdot 3)$ & $\begin{array}{l}108 \cdot 7 \\
(98 \cdot 6 \text { to } \\
124 \cdot 5)\end{array}$ & $\begin{array}{l}91 \cdot 2 \\
(80 \cdot 5 \text { to } \\
113 \cdot 7)\end{array}$ & $\begin{array}{c}-16 \cdot 6(-24 \cdot 8 \text { to } \\
-4 \cdot 2)^{*}\end{array}$ \\
\hline Hepatitis A & $\begin{array}{c}1456 \cdot 7 \\
(673 \cdot 5 \text { to } \\
2476 \cdot 5)\end{array}$ & $\begin{array}{l}1214 \cdot 6 \\
(553 \cdot 8 \text { to } \\
2108 \cdot 4)\end{array}$ & $-17 \cdot 2(-34 \cdot 1$ to $6 \cdot 1)$ & $\begin{array}{l}21 \cdot 3(9 \cdot 8 \\
\text { to } 36 \cdot 1)\end{array}$ & $\begin{array}{l}16 \cdot 6(7 \cdot 6 \\
\text { to } 28 \cdot 8)\end{array}$ & $\begin{array}{c}-22 \cdot 4(-38 \cdot 2 \text { to } \\
-0 \cdot 7)^{*}\end{array}$ \\
\hline Hepatitis B & $\begin{array}{c}2860 \cdot 3 \\
(2022 \cdot 3 \text { to } \\
3868 \cdot 0)\end{array}$ & $\begin{array}{c}2587 \cdot 3 \\
(1839 \cdot 1 \text { to } \\
3512 \cdot 8)\end{array}$ & $-10 \cdot 1(-21 \cdot 5$ to $4 \cdot 9)$ & $\begin{array}{c}46 \cdot 3 \\
(33 \cdot 3 \text { to } \\
61 \cdot 3)\end{array}$ & $\begin{array}{c}37 \cdot 1 \\
(26 \cdot 6 \text { to } \\
49 \cdot 8)\end{array}$ & $\begin{array}{c}-20 \cdot 4(-30 \cdot 0 \text { to } \\
-8 \cdot 0)^{*}\end{array}$ \\
\hline
\end{tabular}




\begin{tabular}{|c|c|c|c|c|c|c|c|}
\hline & & \multicolumn{3}{|c|}{ All ages DALYs (thousands) ${ }^{*}$} & \multicolumn{3}{|c|}{ Age-standardised DALYs (per 100000 )* } \\
\hline & & 2005 & 2013 & Percentage change & 2005 & 2013 & Percentage change \\
\hline & Hepatitis C & $\begin{array}{l}126 \cdot 5 \\
(37 \cdot 9 \text { to } \\
269 \cdot 5)\end{array}$ & $\begin{array}{c}138 \cdot 0 \\
(41 \cdot 4 \text { to } \\
310 \cdot 4)\end{array}$ & $8.1(-8.7$ to 34.5$)$ & $\begin{array}{l}2 \cdot 0(0 \cdot 6 \\
\text { to } 4 \cdot 3)\end{array}$ & $\begin{array}{l}2 \cdot 0(0 \cdot 6 \\
\text { to } 4 \cdot 4)\end{array}$ & $-5 \cdot 0(-19 \cdot 3$ to $17 \cdot 0)$ \\
\hline & Hepatitis E & $\begin{array}{c}2650 \cdot 5 \\
(1991 \cdot 7 \text { to } \\
3421 \cdot 7)\end{array}$ & $\begin{array}{c}2616 \cdot 9 \\
(1962 \cdot 3 \text { to } \\
3508 \cdot 9)\end{array}$ & $-1 \cdot 8(-12 \cdot 8$ to $13 \cdot 4)$ & $\begin{array}{c}39 \cdot 1 \\
(29 \cdot 2 \text { to } \\
50 \cdot 8)\end{array}$ & $\begin{array}{c}35 \cdot 6 \\
(26 \cdot 6 \text { to } \\
47 \cdot 8)\end{array}$ & $-9.5(-19.6$ to 4.5$)$ \\
\hline & eprosy & $\begin{array}{l}36 \cdot 6(24 \cdot 2 \\
\text { to } 51 \cdot 9)\end{array}$ & $\begin{array}{l}39 \cdot 7(26 \cdot 6 \\
\text { to } 56 \cdot 0)\end{array}$ & $8 \cdot 6(2.3 \text { to } 15 \cdot 4)^{*}$ & $\begin{array}{l}0.7(0 \cdot 4 \\
\text { to } 0 \cdot 9)\end{array}$ & $\begin{array}{l}0.6(0 \cdot 4 \\
\text { to } 0 \cdot 9)\end{array}$ & $-8.9(-13.9 \text { to }-3 \cdot 5)^{*}$ \\
\hline & ther infectious diseases & $\begin{array}{c}9181 \cdot 6 \\
(5667 \cdot 4 \text { to } \\
12740 \cdot 2)\end{array}$ & $\begin{array}{c}7660 \cdot 3 \\
(5301 \cdot 6 \text { to } \\
10204 \cdot 5)\end{array}$ & $-14 \cdot 5(-33 \cdot 4$ to $2 \cdot 7)$ & $\begin{array}{l}138 \cdot 6 \\
(87 \cdot 1 \text { to } \\
190 \cdot 1)\end{array}$ & $\begin{array}{c}107 \cdot 4 \\
(74.9 \text { to } \\
142 \cdot 3)\end{array}$ & $\begin{array}{c}-20 \cdot 9(-37 \cdot 7 \text { to } \\
-5 \cdot 1)^{*}\end{array}$ \\
\hline & n-communicable diseases & $\begin{array}{c}1302 \\
199 \cdot 4(1 \\
155437 \cdot 8 \\
\text { to } 1460 \\
687 \cdot 4)\end{array}$ & $\begin{array}{c}1432 \\
938 \cdot 8(1 \\
256004 \cdot 9 \\
\text { to } 1614 \\
026 \cdot 7)\end{array}$ & $10 \cdot 0(7 \cdot 7 \text { to } 12 \cdot 8)^{*}$ & $\begin{array}{c}22873 \cdot 8 \\
(20458 \cdot 5 \\
\text { to } 25 \\
487 \cdot 7)\end{array}$ & $\begin{array}{c}21452 \cdot 8 \\
(18880 \cdot 3 \\
\text { to } 24 \\
078 \cdot 4)\end{array}$ & $-6 \cdot 3(-8 \cdot 3 \text { to }-3 \cdot 8)^{*}$ \\
\hline & Neoplasms & $\begin{array}{c}180409 \cdot 6 \\
(175 \\
482 \cdot 3 \text { to } \\
185 \\
592 \cdot 2)\end{array}$ & $\begin{array}{c}197093 \cdot 5 \\
(189 \\
237 \cdot 0 \text { to } \\
206 \\
258 \cdot 5)\end{array}$ & $9 \cdot 3(4.9 \text { to } 13.9)^{*}$ & $\begin{array}{c}3289 \cdot 8 \\
(\mathbf{3 1 9 6} \cdot 1 \\
\text { to } \\
\mathbf{3 3 8 4} \cdot 1)\end{array}$ & $\begin{array}{c}3001 \cdot 7 \\
(2881 \cdot 6 \\
\text { to } \\
3136 \cdot 4)\end{array}$ & $-8 \cdot 7(-12 \cdot 3 \text { to }-5 \cdot 0)^{*}$ \\
\hline & Oesophageal cancer & $\begin{array}{c}8905 \cdot 2 \\
(7787 \cdot 5 \text { to } \\
10237 \cdot 4)\end{array}$ & $\begin{array}{c}9843 \cdot 1 \\
(8655 \cdot 5 \text { to } \\
11620 \cdot 1)\end{array}$ & $10.3(1.9 \text { to } 20.3)^{*}$ & $\begin{array}{c}168 \cdot 8 \\
(148 \cdot 2 \text { to } \\
193 \cdot 7)\end{array}$ & $\begin{array}{c}152 \cdot 3 \\
(134 \cdot 0 \text { to } \\
180 \cdot 1)\end{array}$ & $-9.9(-16.7 \text { to }-1.7)^{*}$ \\
\hline & Stomach cancer & $\begin{array}{c}19059 \cdot 1 \\
(18331 \cdot 4 \\
\text { to } 19 \\
926 \cdot 9)\end{array}$ & $\begin{array}{c}17906 \cdot 5 \\
(16863 \cdot 7 \\
\text { to } 19 \\
067 \cdot 8)\end{array}$ & $-6 \cdot 0(-11.5 \text { to }-0 \cdot 6)^{*}$ & $\begin{array}{c}357 \cdot 4 \\
(343 \cdot 9 \text { to } \\
373 \cdot 8)\end{array}$ & $\begin{array}{l}277.7 \\
(261.5 \text { to } \\
295.9)\end{array}$ & $\begin{array}{c}-22 \cdot 2(-26 \cdot 8 \text { to } \\
-17 \cdot 9)^{*}\end{array}$ \\
\hline & Liver cancer & $\begin{array}{c}19175 \cdot 3 \\
(18331 \cdot 6 \\
\text { to } 20 \\
085 \cdot 5)\end{array}$ & $\begin{array}{c}20888 \cdot 7 \\
(19321 \cdot 9 \\
\text { to } 22 \\
518 \cdot 1)\end{array}$ & $9 \cdot 2(-0 \cdot 2$ to $17 \cdot 7)$ & $\begin{array}{c}344 \cdot 0 \\
(329 \cdot 1 \text { to } \\
360 \cdot 4)\end{array}$ & $\begin{array}{c}313 \cdot 0 \\
(289 \cdot 9 \text { to } \\
336 \cdot 5)\end{array}$ & $-8.9(-16.3 \text { to }-1.9)^{*}$ \\
\hline B & Liver cancer due to hepatitis & $\begin{array}{c}8198.9 \\
(7558 \cdot 5 \text { to } \\
8758.0)\end{array}$ & $\begin{array}{c}8590 \cdot 9 \\
(7761 \cdot 8 \text { to } \\
9462 \cdot 3)\end{array}$ & $4 \cdot 8(-6 \cdot 5$ to $16 \cdot 2)$ & $\begin{array}{c}143.6 \\
(132 \cdot 7 \text { to } \\
153 \cdot 2)\end{array}$ & $\begin{array}{c}126 \cdot 2 \\
(114 \cdot 1 \text { to } \\
138 \cdot 7)\end{array}$ & $\begin{array}{c}-12 \cdot 1(-21 \cdot 3 \text { to } \\
-2 \cdot 7)^{*}\end{array}$ \\
\hline $\mathrm{C}$ & Liver cancer due to hepatitis & $\begin{array}{c}5902 \cdot 3 \\
(5484 \cdot 4 \text { to } \\
6445 \cdot 1)\end{array}$ & $\begin{array}{c}7967 \cdot 1 \\
(7271 \cdot 6 \text { to } \\
8807 \cdot 4)\end{array}$ & $35.1(21.9 \text { to } 47.7)^{*}$ & $\begin{array}{c}109 \cdot 0 \\
(101 \cdot 5 \text { to } \\
118 \cdot 8)\end{array}$ & $\begin{array}{c}121 \cdot 4 \\
(111 \cdot 1 \text { to } \\
133 \cdot 6)\end{array}$ & $11.5(0.7 \text { to } 21.5)^{*}$ \\
\hline use & Liver cancer due to alcohol & $\begin{array}{c}2450 \cdot 5 \\
(2239 \cdot 5 \text { to } \\
2675 \cdot 5)\end{array}$ & $\begin{array}{c}1980 \cdot 4 \\
(1813 \cdot 1 \text { to } \\
2189 \cdot 7)\end{array}$ & $\begin{array}{c}-19 \cdot 1(-27.9 \text { to } \\
-9 \cdot 7)^{*}\end{array}$ & $\begin{array}{c}46 \cdot 1 \\
(42 \cdot 2 \text { to } \\
50 \cdot 2)\end{array}$ & $\begin{array}{c}30 \cdot 7 \\
(28 \cdot 2 \text { to } \\
33 \cdot 9)\end{array}$ & $\begin{array}{c}-33 \cdot 2(-40 \cdot 2 \text { to } \\
-25 \cdot 7)^{*}\end{array}$ \\
\hline causes & Liver cancer due to other & $\begin{array}{c}2623 \cdot 7 \\
(2366 \cdot 6 \text { to } \\
2881 \cdot 3)\end{array}$ & $\begin{array}{c}2350 \cdot 2 \\
(2098 \cdot 1 \text { to } \\
2595 \cdot 8)\end{array}$ & $-9 \cdot 4(-24 \cdot 9$ to $2 \cdot 8)$ & $\begin{array}{c}45 \cdot 3 \\
(40 \cdot 9 \text { to } \\
49 \cdot 7)\end{array}$ & $\begin{array}{c}34 \cdot 6 \\
(31 \cdot 0 \text { to } \\
38 \cdot 3)\end{array}$ & $\begin{array}{c}-22 \cdot 8(-35 \cdot 9 \text { to } \\
-12 \cdot 4)^{*}\end{array}$ \\
\hline & Larynx cancer & $\begin{array}{l}2075 \cdot 5 \\
(1812 \cdot 6 \text { to } \\
2544 \cdot 4)\end{array}$ & $\begin{array}{l}2136 \cdot 7 \\
(1815 \cdot 5 \text { to } \\
2620 \cdot 1)\end{array}$ & $3 \cdot 0(-3 \cdot 8$ to $10 \cdot 0)$ & $\begin{array}{c}38 \cdot 6 \\
(33 \cdot 6 \text { to } \\
47 \cdot 1)\end{array}$ & $\begin{array}{c}32 \cdot 6 \\
(27 \cdot 8 \text { to } \\
39 \cdot 9)\end{array}$ & $\begin{array}{c}-15 \cdot 5(-20 \cdot 9 \text { to } \\
-9 \cdot 9)^{*}\end{array}$ \\
\hline cancer & Tracheal, bronchus, and lung & $\begin{array}{c}30791 \cdot 6 \\
(29492 \cdot 6 \\
\text { to } 31 \\
587 \cdot 1)\end{array}$ & $\begin{array}{c}34732 \cdot 9 \\
(33042 \cdot 6 \\
\text { to } 36 \\
328 \cdot 1)\end{array}$ & $12.9(6 \cdot 6 \text { to } 19 \cdot 1)^{*}$ & $\begin{array}{l}586 \cdot 7 \\
(562 \cdot 2 \text { to } \\
601 \cdot 6)\end{array}$ & $\begin{array}{l}542 \cdot 8 \\
(516 \cdot 4 \text { to } \\
567 \cdot 1)\end{array}$ & $-7 \cdot 4(-12 \cdot 4 \text { to }-2 \cdot 5)^{*}$ \\
\hline & Breast cancer & $\begin{array}{c}11762 \cdot 5 \\
(10713 \cdot 2 \\
\text { to } 13 \\
178 \cdot 0)\end{array}$ & $\begin{array}{c}13258 \cdot 7 \\
(12105 \cdot 4 \\
\text { to } 14 \\
558 \cdot 1)\end{array}$ & $13.0(4.5 \text { to } 19.8)^{*}$ & $\begin{array}{c}209 \cdot 8 \\
(190 \cdot 6 \text { to } \\
234 \cdot 6)\end{array}$ & $\begin{array}{c}196 \cdot 4 \\
(178 \cdot 1 \text { to } \\
215 \cdot 5)\end{array}$ & $-6 \cdot 1(-13 \cdot 0 \text { to }-0 \cdot 7)^{*}$ \\
\hline
\end{tabular}




\begin{tabular}{|c|c|c|c|c|c|c|c|}
\hline & & \multicolumn{3}{|c|}{ All ages DALYs (thousands) ${ }^{*}$} & \multicolumn{3}{|c|}{ Age-standardised DALYs (per 100 000) } \\
\hline & & 2005 & 2013 & Percentage change & 2005 & 2013 & Percentage change \\
\hline & Cervical cancer & $\begin{array}{c}6775 \cdot 6 \\
(5813 \cdot 9 \text { to } \\
7591 \cdot 5)\end{array}$ & $\begin{array}{l}6914 \cdot 7 \\
(5774 \cdot 5 \text { to } \\
7589 \cdot 1)\end{array}$ & $2 \cdot 1(-5 \cdot 7$ to $9 \cdot 5)$ & $\begin{array}{c}118.0 \\
(101 \cdot 3 \text { to } \\
131 \cdot 9)\end{array}$ & $\begin{array}{c}100 \cdot 9 \\
(84 \cdot 4 \text { to } \\
110 \cdot 5)\end{array}$ & $\begin{array}{c}-14.4(-20 \cdot 7 \text { to } \\
-8 \cdot 2)^{*}\end{array}$ \\
\hline & Uterine cancer & $\begin{array}{c}1526 \cdot 9 \\
(1184 \cdot 9 \text { to } \\
1824 \cdot 8)\end{array}$ & $\begin{array}{c}1660 \cdot 9 \\
(1276 \cdot 3 \text { to } \\
1961 \cdot 6)\end{array}$ & $8 \cdot 1(-1 \cdot 1$ to $21 \cdot 3)$ & $\begin{array}{c}28 \cdot 5 \\
(22 \cdot 3 \text { to } \\
34 \cdot 0)\end{array}$ & $\begin{array}{c}25 \cdot 4 \\
(19 \cdot 6 \text { to } \\
30 \cdot 0)\end{array}$ & $\begin{array}{c}-11.3(-18.6 \text { to } \\
-0.7)^{*}\end{array}$ \\
\hline & Prostate cancer & $\begin{array}{c}3812 \cdot 1 \\
(3236 \cdot 1 \text { to } \\
4802 \cdot 3)\end{array}$ & $\begin{array}{c}4768 \cdot 8 \\
(4067 \cdot 0 \text { to } \\
6034 \cdot 1)\end{array}$ & $25.0(19.3 \text { to } 31.6)^{*}$ & $\begin{array}{c}80 \cdot 2 \\
(68 \cdot 3 \text { to } \\
100 \cdot 9)\end{array}$ & $\begin{array}{l}81 \cdot 3 \\
(69 \cdot 2 \text { to } \\
103 \cdot 0)\end{array}$ & $1 \cdot 3(-3 \cdot 3$ to $6 \cdot 7)$ \\
\hline & Colon and rectum cancer & $\begin{array}{c}13747.9 \\
(13378.9 \\
\text { to } 14 \\
138.6)\end{array}$ & $\begin{array}{c}15794 \cdot 1 \\
(15165 \cdot 3 \\
\text { to } 16 \\
421 \cdot 4)\end{array}$ & $14.9(10 \cdot 8 \text { to } 19 \cdot 1)^{*}$ & $\begin{array}{c}261.6 \\
(254.4 \text { to } \\
268.9)\end{array}$ & $\begin{array}{l}246 \cdot 7 \\
(237 \cdot 0 \text { to } \\
256 \cdot 2)\end{array}$ & $-5 \cdot 7(-9 \cdot 0 \text { to }-2 \cdot 4)^{*}$ \\
\hline & Lip and oral cavity cancer & $\begin{array}{c}2963 \cdot 9 \\
(2620 \cdot 9 \text { to } \\
3442 \cdot 8)\end{array}$ & $\begin{array}{c}3589 \cdot 3 \\
(3031 \cdot 8 \text { to } \\
4109 \cdot 0)\end{array}$ & $21.1(9.8 \text { to } 32.1)^{*}$ & $\begin{array}{c}53 \cdot 8 \\
(47 \cdot 7 \text { to } \\
62 \cdot 6)\end{array}$ & $\begin{array}{c}53 \cdot 8 \\
(45 \cdot 5 \text { to } \\
61 \cdot 7)\end{array}$ & $0.0(-9 \cdot 1$ to $8 \cdot 7)$ \\
\hline & Nasopharynx cancer & $\begin{array}{c}2034 \cdot 5 \\
(1831 \cdot 3 \text { to } \\
2318 \cdot 1)\end{array}$ & $\begin{array}{c}1933 \cdot 7 \\
(1723 \cdot 7 \text { to } \\
2211 \cdot 8)\end{array}$ & $-5 \cdot 0(-13 \cdot 1$ to $4 \cdot 1)$ & $\begin{array}{c}34 \cdot 5 \\
(31 \cdot 2 \text { to } \\
39 \cdot 3)\end{array}$ & $\begin{array}{c}27.9 \\
(24.9 \text { to } \\
31.9)\end{array}$ & $\begin{array}{c}-19 \cdot 3(-26 \cdot 1 \text { to } \\
-11 \cdot 7)^{*}\end{array}$ \\
\hline & Other pharynx cancer & $\begin{array}{c}1732 \cdot 8 \\
(1545 \cdot 7 \text { to } \\
1880 \cdot 0)\end{array}$ & $\begin{array}{c}2137 \cdot 7 \\
(1832 \cdot 0 \text { to } \\
2368 \cdot 2)\end{array}$ & $23 \cdot 3(10 \cdot 9 \text { to } 36 \cdot 4)^{*}$ & $\begin{array}{c}31 \cdot 5 \\
(28 \cdot 2 \text { to } \\
34 \cdot 2)\end{array}$ & $\begin{array}{c}31 \cdot 8 \\
(27 \cdot 3 \text { to } \\
35 \cdot 2)\end{array}$ & $1.2(-9.1$ to 11.9$)$ \\
\hline cancer & Gallbladder and biliary tract & $\begin{array}{c}2550 \cdot 4 \\
(2310 \cdot 8 \text { to } \\
2841 \cdot 4)\end{array}$ & $\begin{array}{c}2701 \cdot 1 \\
(2338 \cdot 8 \text { to } \\
2977 \cdot 8)\end{array}$ & $6 \cdot 3(-2 \cdot 9$ to $14 \cdot 1)$ & $\begin{array}{c}49 \cdot 0 \\
(44 \cdot 4 \text { to } \\
54 \cdot 8)\end{array}$ & $\begin{array}{c}42 \cdot 4 \\
(36 \cdot 9 \text { to } \\
47 \cdot 0)\end{array}$ & $\begin{array}{c}-13 \cdot 0(-20 \cdot 7 \text { to } \\
-6 \cdot 7)^{*}\end{array}$ \\
\hline & Pancreatic cancer & $\begin{array}{c}5704 \cdot 7 \\
(5557 \cdot 7 \text { to } \\
5841 \cdot 8)\end{array}$ & $\begin{array}{c}7029 \cdot 1 \\
(6775 \cdot 5 \text { to } \\
7276 \cdot 7)\end{array}$ & $23.2(19.2 \text { to } 27.5)^{*}$ & $\begin{array}{c}109 \cdot 5 \\
(106 \cdot 6 \text { to } \\
112 \cdot 1)\end{array}$ & $\begin{array}{c}110 \cdot 2 \\
(106 \cdot 3 \text { to } \\
114 \cdot 1)\end{array}$ & $0 \cdot 6(-2 \cdot 7$ to $4 \cdot 0)$ \\
\hline & Malignant skin melanoma & $\begin{array}{c}1394 \cdot 8 \\
(1102 \cdot 7 \text { to } \\
1877 \cdot 5)\end{array}$ & $\begin{array}{c}1555 \cdot 5 \\
(1227 \cdot 7 \text { to } \\
2089 \cdot 3)\end{array}$ & $12.0(3.5 \text { to } 18.7)^{*}$ & $\begin{array}{c}24 \cdot 8 \\
(19 \cdot 3 \text { to } \\
33 \cdot 5)\end{array}$ & $\begin{array}{l}23 \cdot 2 \\
(18 \cdot 1 \text { to } \\
31 \cdot 1)\end{array}$ & $-6 \cdot 1(-13 \cdot 0 \text { to }-0 \cdot 8)^{*}$ \\
\hline & Non-melanoma skin cancer & $\begin{array}{c}724 \cdot 2 \\
(602 \cdot 1 \text { to } \\
903 \cdot 6)\end{array}$ & $\begin{array}{c}816 \cdot 5 \\
(682 \cdot 2 \text { to } \\
1039 \cdot 9)\end{array}$ & $12 \cdot 4(6 \cdot 7 \text { to } 20 \cdot 1)^{*}$ & $\begin{array}{l}13.7 \\
(11.4 \text { to } \\
16 \cdot 9)\end{array}$ & $\begin{array}{l}12 \cdot 9 \\
(10 \cdot 8 \text { to } \\
16 \cdot 3)\end{array}$ & $-6 \cdot 2(-10 \cdot 7$ to $0 \cdot 0)$ \\
\hline & Ovarian cancer & $\begin{array}{c}3541 \cdot 7 \\
(3324 \cdot 8 \text { to } \\
3725 \cdot 9)\end{array}$ & $\begin{array}{c}4056 \cdot 5 \\
(3794 \cdot 9 \text { to } \\
4400 \cdot 2)\end{array}$ & $14 \cdot 5(7 \cdot 0 \text { to } 23 \cdot 1)^{*}$ & $\begin{array}{l}64 \cdot 1 \\
(60 \cdot 2 \text { to } \\
67 \cdot 3)\end{array}$ & $\begin{array}{c}60 \cdot 6 \\
(56 \cdot 6 \text { to } \\
65 \cdot 5)\end{array}$ & $-5 \cdot 5(-11 \cdot 4$ to $1 \cdot 3)$ \\
\hline & Testicular cancer & $\begin{array}{c}354.9 \\
(284.9 \text { to } \\
439 \cdot 2)\end{array}$ & $\begin{array}{c}378 \cdot 7 \\
(284 \cdot 3 \text { to } \\
470 \cdot 6)\end{array}$ & $7 \cdot 2(-3.8$ to $16 \cdot 4)$ & $\begin{array}{l}5 \cdot 5(4 \cdot 4 \\
\text { to } 6 \cdot 8)\end{array}$ & $\begin{array}{l}5 \cdot 2(3 \cdot 9 \\
\text { to } 6 \cdot 5)\end{array}$ & $-4 \cdot 4(-14 \cdot 2$ to $3 \cdot 6)$ \\
\hline & Kidney cancer & $\begin{array}{c}2810 \cdot 2 \\
(2675 \cdot 3 \text { to } \\
2923 \cdot 1)\end{array}$ & $\begin{array}{c}3150 \cdot 3 \\
(2988 \cdot 6 \text { to } \\
3320 \cdot 7)\end{array}$ & $12 \cdot 2(5 \cdot 8 \text { to } 18 \cdot 1)^{*}$ & $\begin{array}{c}51 \cdot 8 \\
(49 \cdot 2 \text { to } \\
54 \cdot 0)\end{array}$ & $\begin{array}{c}48 \cdot 3 \\
(45 \cdot 8 \text { to } \\
50 \cdot 8)\end{array}$ & $-6 \cdot 7(-12 \cdot 0$ to $-2 \cdot 2)$ * \\
\hline & Bladder cancer & $\begin{array}{c}2987 \cdot 4 \\
(2743 \cdot 2 \text { to } \\
3270 \cdot 2)\end{array}$ & $\begin{array}{c}3139.9 \\
(2868 \cdot 8 \text { to } \\
3479 \cdot 6)\end{array}$ & $4.9(0.8 \text { to } 10 \cdot 3)^{*}$ & $\begin{array}{c}59 \cdot 0 \\
(54 \cdot 0 \text { to } \\
64 \cdot 4)\end{array}$ & $\begin{array}{c}50 \cdot 6 \\
(46 \cdot 3 \text { to } \\
56 \cdot 0)\end{array}$ & $\begin{array}{c}-14.4(-17.6 \text { to } \\
-10 \cdot 0)^{*}\end{array}$ \\
\hline cancer & Brain and nervous system & $\begin{array}{c}6163 \cdot 4 \\
(5120 \cdot 0 \text { to } \\
7002 \cdot 9)\end{array}$ & $\begin{array}{c}6692 \cdot 2 \\
(5592 \cdot 3 \text { to } \\
7765 \cdot 2)\end{array}$ & $8.4(2.3 \text { to } 15.4)^{*}$ & $\begin{array}{c}102 \cdot 6 \\
(85 \cdot 2 \text { to } \\
115 \cdot 8)\end{array}$ & $\begin{array}{c}96 \cdot 8 \\
(80 \cdot 9 \text { to } \\
112 \cdot 1)\end{array}$ & $-5 \cdot 7(-10 \cdot 8$ to 0.4$)$ \\
\hline & Thyroid cancer & $\begin{array}{l}764 \cdot 5 \\
(673 \cdot 5 \text { to } \\
870 \cdot 8)\end{array}$ & $\begin{array}{c}851 \cdot 9 \\
(739 \cdot 8 \text { to } \\
983 \cdot 2)\end{array}$ & $12.3(2.4 \text { to } 19 \cdot 2)^{*}$ & $\begin{array}{c}14 \cdot 0 \\
(12 \cdot 4 \text { to } \\
16 \cdot 0)\end{array}$ & $\begin{array}{c}13.0 \\
(11 \cdot 3 \text { to } \\
14.9)\end{array}$ & $-6 \cdot 7(-14 \cdot 4 \text { to }-1 \cdot 1)^{*}$ \\
\hline & Mesothelioma & $\begin{array}{c}504 \cdot 0 \\
(444 \cdot 0 \text { to } \\
581 \cdot 2)\end{array}$ & $\begin{array}{l}763 \cdot 5 \\
(686 \cdot 2 \text { to } \\
864 \cdot 4)\end{array}$ & $51.8(40 \cdot 0 \text { to } 63.4)^{*}$ & $\begin{array}{l}9 \cdot 5(8 \cdot 3 \\
\text { to } 11 \cdot 0)\end{array}$ & $\begin{array}{l}11 \cdot 8 \\
(10 \cdot 5 \text { to } \\
13 \cdot 4)\end{array}$ & $24.4(14.7 \text { to } 33 \cdot 8)^{*}$ \\
\hline & Hodgkin's lymphoma & $\begin{array}{c}1126 \cdot 5 \\
(1027 \cdot 5 \text { to } \\
1378.4)\end{array}$ & $\begin{array}{c}989.6 \\
(867.9 \text { to } \\
1304 \cdot 0)\end{array}$ & $-13 \cdot 5(-21 \cdot 9$ to $4 \cdot 2)$ & $\begin{array}{l}17 \cdot 7 \\
(16 \cdot 2 \text { to } \\
21 \cdot 8)\end{array}$ & $\begin{array}{c}14 \cdot 0 \\
(12 \cdot 3 \text { to } \\
18 \cdot 4)\end{array}$ & $\begin{array}{c}-22 \cdot 3(-29 \cdot 4 \text { to } \\
-7 \cdot 0)^{*}\end{array}$ \\
\hline
\end{tabular}




\begin{tabular}{|c|c|c|c|c|c|c|}
\hline & \multicolumn{3}{|c|}{ All ages DALYs (thousands) ${ }^{*}$} & \multicolumn{3}{|c|}{ Age-standardised DALYs (per 100000$)^{*}$} \\
\hline & 2005 & 2013 & Percentage change & 2005 & 2013 & Percentage change \\
\hline Non-Hodgkin lymphoma & $\begin{array}{c}5627 \cdot 7 \\
(4867 \cdot 0 \text { to } \\
6301 \cdot 8)\end{array}$ & $\begin{array}{c}6412 \cdot 8 \\
(5495 \cdot 7 \text { to } \\
7202 \cdot 7)\end{array}$ & $14 \cdot 6(5 \cdot 7 \text { to } 21 \cdot 1)^{*}$ & $\begin{array}{c}96 \cdot 9 \\
(83 \cdot 1 \text { to } \\
107 \cdot 1)\end{array}$ & $\begin{array}{c}94 \cdot 9 \\
(81 \cdot 2 \text { to } \\
106 \cdot 2)\end{array}$ & $-1 \cdot 4(-8.9$ to $4 \cdot 0)$ \\
\hline Multiple myeloma & $\begin{array}{c}1384 \cdot 2 \\
(1174 \cdot 0 \text { to } \\
1635 \cdot 6)\end{array}$ & $\begin{array}{c}1661 \cdot 5 \\
(1397 \cdot 5 \text { to } \\
1964 \cdot 8)\end{array}$ & $20 \cdot 3(13 \cdot 4 \text { to } 25 \cdot 0)^{*}$ & $\begin{array}{c}26 \cdot 6 \\
(22 \cdot 5 \text { to } \\
31 \cdot 5)\end{array}$ & $\begin{array}{c}26 \cdot 1 \\
(21 \cdot 8 \text { to } \\
31 \cdot 0)\end{array}$ & $-1 \cdot 7(-7 \cdot 3$ to $2 \cdot 2)$ \\
\hline Leukaemia & $\begin{array}{l}9384 \cdot 9 \\
(9081 \cdot 5 \text { to } \\
9744 \cdot 4)\end{array}$ & $\begin{array}{c}9301 \cdot 0 \\
(8869 \cdot 0 \text { to } \\
9752 \cdot 8)\end{array}$ & $-0 \cdot 7(-6 \cdot 0$ to $3 \cdot 6)$ & $\begin{array}{c}150 \cdot 0 \\
(145 \cdot 2 \text { to } \\
155 \cdot 3)\end{array}$ & $\begin{array}{c}133 \cdot 7 \\
(127 \cdot 6 \text { to } \\
140 \cdot 1)\end{array}$ & $\begin{array}{c}-10 \cdot 8(-15 \cdot 2 \text { to } \\
-7 \cdot 0)^{*}\end{array}$ \\
\hline Other neoplasms & $\begin{array}{c}11023 \cdot 4 \\
(10179 \cdot 5 \\
\text { to } 12 \\
208 \cdot 5)\end{array}$ & $\begin{array}{c}12027 \cdot 7 \\
(10693 \cdot 3 \\
\text { to } 13 \\
370 \cdot 7)\end{array}$ & $10 \cdot 3(-3 \cdot 1$ to $17 \cdot 1)$ & $\begin{array}{c}182 \cdot 0 \\
(167 \cdot 7 \text { to } \\
200 \cdot 3)\end{array}$ & $\begin{array}{c}175 \cdot 3 \\
(156 \cdot 1 \text { to } \\
194 \cdot 1)\end{array}$ & $-2 \cdot 7(-13 \cdot 8$ to $2 \cdot 9)$ \\
\hline Cardiovascular diseases & $\begin{array}{c}308887 \cdot 0 \\
(294 \\
356 \cdot 7 \text { to } \\
324 \\
066 \cdot 8)\end{array}$ & $\begin{array}{c}329705 \cdot 6 \\
(311 \\
188 \cdot 8 \text { to } \\
348 \\
206 \cdot 2)\end{array}$ & $6.7(2 \cdot 6 \text { to } 11 \cdot 7)^{*}$ & $\begin{array}{c}5907 \cdot 1 \\
(5641 \cdot 6 \\
\text { to } \\
6181 \cdot 0)\end{array}$ & $\begin{array}{c}5206 \cdot 3 \\
(4924 \cdot 1 \\
\text { to } \\
5485 \cdot 9)\end{array}$ & $\begin{array}{c}-11.9(-15 \cdot 1 \text { to } \\
-8 \cdot 0)^{*}\end{array}$ \\
\hline Rheumatic heart disease & $\begin{array}{l}10103 \cdot 6 \\
(8576 \cdot 3 \text { to } \\
12982 \cdot 8)\end{array}$ & $\begin{array}{c}9517 \cdot 7 \\
(7867 \cdot 8 \text { to } \\
11950 \cdot 8)\end{array}$ & $-5 \cdot 9(-13 \cdot 9$ to $3 \cdot 4)$ & $\begin{array}{c}170 \cdot 0 \\
(144 \cdot 4 \text { to } \\
219 \cdot 1)\end{array}$ & $\begin{array}{c}138 \cdot 9 \\
(115 \cdot 1 \text { to } \\
174 \cdot 2)\end{array}$ & $\begin{array}{c}-18 \cdot 4(-25 \cdot 5 \text { to } \\
-10 \cdot 3)^{*}\end{array}$ \\
\hline Ischaemic heart disease & $\begin{array}{c}138547 \cdot 2 \\
(127 \\
675 \cdot 5 \text { to } \\
149 \\
798 \cdot 3)\end{array}$ & $\begin{array}{c}150238 \cdot 6 \\
(135 \\
388 \cdot 5 \text { to } \\
162 \\
458 \cdot 7)\end{array}$ & $8.4(2.9 \text { to } 15 \cdot 0)^{*}$ & $\begin{array}{c}2670 \cdot 7 \\
(2461 \cdot 1 \\
\text { to } \\
2880 \cdot 1)\end{array}$ & $\begin{array}{c}2375 \cdot 9 \\
(2142 \cdot 4 \\
\text { to } \\
2565 \cdot 1)\end{array}$ & $\begin{array}{c}-11 \cdot 1(-15 \cdot 2 \text { to } \\
-6 \cdot 0)^{*}\end{array}$ \\
\hline Cerebrovascular disease & $\begin{array}{c}107737 \cdot 1 \\
(99331 \cdot 7 \\
\text { to } 116 \\
802 \cdot 3)\end{array}$ & $\begin{array}{c}112878 \cdot 9 \\
(104 \\
002 \cdot 3 \text { to } \\
124 \\
567 \cdot 7)\end{array}$ & $4.7(0.2 \text { to } 9 \cdot 6)^{*}$ & $\begin{array}{c}2096 \cdot 8 \\
(1934 \cdot 1 \\
\text { to } \\
2266 \cdot 5)\end{array}$ & $\begin{array}{c}1806 \cdot 9 \\
(1667 \cdot 4 \\
\text { to } \\
1991 \cdot 7)\end{array}$ & $\begin{array}{c}-13 \cdot 9(-17 \cdot 5 \text { to } \\
-9 \cdot 9)^{*}\end{array}$ \\
\hline Ischaemic stroke & $\begin{array}{c}44730 \cdot 9 \\
(38134 \cdot 8 \\
\text { to } 49 \\
037 \cdot 1)\end{array}$ & $\begin{array}{c}47424 \cdot 7 \\
(40537 \cdot 5 \\
\text { to } 52 \\
211 \cdot 8)\end{array}$ & $6.0(0.8 \text { to } 11.0)^{*}$ & $\begin{array}{c}920 \cdot 4 \\
(787 \cdot 2 \text { to } \\
1007 \cdot 9)\end{array}$ & $\begin{array}{c}791 \cdot 3 \\
(678 \cdot 0 \text { to } \\
868 \cdot 8)\end{array}$ & $\begin{array}{c}-14 \cdot 0(-18 \cdot 2 \text { to } \\
-10 \cdot 0)^{*}\end{array}$ \\
\hline Haemorrhagic stroke & $\begin{array}{c}63006 \cdot 2 \\
(57306 \cdot 5 \\
\text { to } 70 \\
880 \cdot 3)\end{array}$ & $\begin{array}{c}65454 \cdot 2 \\
(59497 \cdot 4 \\
\text { to } 74 \\
654 \cdot 7)\end{array}$ & $3 \cdot 8(-1 \cdot 6$ to $10 \cdot 1)$ & $\begin{array}{c}1176 \cdot 4 \\
(1068 \cdot 3 \\
\text { to } \\
1325 \cdot 3)\end{array}$ & $\begin{array}{c}1015 \cdot 6 \\
(923 \cdot 2 \text { to } \\
1163 \cdot 2)\end{array}$ & $\begin{array}{c}-13 \cdot 8(-18 \cdot 2 \text { to } \\
-8 \cdot 5)^{*}\end{array}$ \\
\hline Hypertensive heart disease & $\begin{array}{c}16427 \cdot 8 \\
(13746 \cdot 5 \\
\text { to } 19 \\
904 \cdot 3)\end{array}$ & $\begin{array}{c}19248 \cdot 1 \\
(15498 \cdot 3 \\
\text { to } 22 \\
588 \cdot 0)\end{array}$ & $17.7(6 \cdot 2 \text { to } 27.9)^{*}$ & $\begin{array}{c}320 \cdot 7 \\
(269 \cdot 1 \text { to } \\
388 \cdot 9)\end{array}$ & $\begin{array}{c}308 \cdot 0 \\
(248 \cdot 4 \text { to } \\
360 \cdot 3)\end{array}$ & $-3 \cdot 6(-12 \cdot 9$ to $4 \cdot 7)$ \\
\hline Cardiomyopathy and myocarditis & $\begin{array}{c}12876 \cdot 8 \\
(10178 \cdot 6 \\
\text { to } 14 \\
361 \cdot 3)\end{array}$ & $\begin{array}{c}12472 \cdot 7 \\
(10209 \cdot 8 \\
\text { to } 14 \\
036 \cdot 3)\end{array}$ & $-3 \cdot 5(-8 \cdot 0$ to $5 \cdot 8)$ & $\begin{array}{c}220 \cdot 3 \\
(176 \cdot 5 \text { to } \\
244 \cdot 8)\end{array}$ & $\begin{array}{c}184 \cdot 3 \\
(151 \cdot 9 \text { to } \\
207 \cdot 8)\end{array}$ & $\begin{array}{c}-16 \cdot 7(-20 \cdot 3 \text { to } \\
-9 \cdot 5)^{*}\end{array}$ \\
\hline Atrial fibrillation and flutter & $\begin{array}{c}1477 \cdot 2 \\
(1238 \cdot 5 \text { to } \\
1748 \cdot 3)\end{array}$ & $\begin{array}{c}1888 \cdot 7 \\
(1590 \cdot 0 \text { to } \\
2224 \cdot 9)\end{array}$ & $28.1(20 \cdot 9 \text { to } 34 \cdot 6)^{*}$ & $\begin{array}{c}32 \cdot 1 \\
(27 \cdot 0 \text { to } \\
37 \cdot 9)\end{array}$ & $\begin{array}{c}32 \cdot 6 \\
(27 \cdot 5 \text { to } \\
38 \cdot 2)\end{array}$ & $1 \cdot 6(-4 \cdot 1$ to $6 \cdot 9)$ \\
\hline Aortic aneurysm & $\begin{array}{c}2404 \cdot 4 \\
(1973 \cdot 3 \text { to } \\
2797 \cdot 7)\end{array}$ & $\begin{array}{c}2652 \cdot 7 \\
(2217 \cdot 4 \text { to } \\
3109 \cdot 6)\end{array}$ & $10 \cdot 2(5 \cdot 5 \text { to } 16 \cdot 3)^{*}$ & $\begin{array}{c}46 \cdot 5 \\
(38 \cdot 3 \text { to } \\
54 \cdot 3)\end{array}$ & $\begin{array}{c}42 \cdot 2 \\
(35 \cdot 2 \text { to } \\
49 \cdot 4)\end{array}$ & $-9 \cdot 4(-13 \cdot 1 \text { to }-4 \cdot 7)^{*}$ \\
\hline Peripheral vascular disease & $\begin{array}{c}510 \cdot 7 \\
(438 \cdot 1 \text { to } \\
599 \cdot 2)\end{array}$ & $\begin{array}{c}596 \cdot 1 \\
(515 \cdot 2 \text { to } \\
705 \cdot 5)\end{array}$ & $16.8(10.6 \text { to } 23.2)^{*}$ & $\begin{array}{l}11 \cdot 0(9 \cdot 4 \\
\text { to } 12 \cdot 9)\end{array}$ & $\begin{array}{l}10 \cdot 2(8 \cdot 8 \\
\text { to } 12 \cdot 1)\end{array}$ & $-7 \cdot 1(-12 \cdot 1 \text { to }-2 \cdot 1)^{*}$ \\
\hline
\end{tabular}




\begin{tabular}{|c|c|c|c|c|c|c|}
\hline & \multicolumn{3}{|c|}{ All ages DALYs (thousands) ${ }^{*}$} & \multicolumn{3}{|c|}{$\underline{\text { Age-standardised DALYs (per } 100000 \text { ) }}$} \\
\hline & 2005 & 2013 & Percentage change & 2005 & 2013 & Percentage change \\
\hline Endocarditis & $\begin{array}{c}1769 \cdot 6 \\
(1301 \cdot 1 \text { to } \\
2161 \cdot 5)\end{array}$ & $\begin{array}{c}1913 \cdot 5 \\
(1420 \cdot 8 \text { to } \\
2342 \cdot 1)\end{array}$ & $7.9(0.2 \text { to } 17.9)^{*}$ & $\begin{array}{c}29 \cdot 8 \\
(22 \cdot 1 \text { to } \\
36 \cdot 3)\end{array}$ & $\begin{array}{c}28 \cdot 0 \\
(20 \cdot 8 \text { to } \\
34 \cdot 4)\end{array}$ & $-6 \cdot 4(-12 \cdot 7$ to $2 \cdot 4)$ \\
\hline $\begin{array}{l}\text { Other cardiovascular and } \\
\text { circulatory diseases }\end{array}$ & $\begin{array}{c}17032 \cdot 8 \\
(14236 \cdot 3 \\
\text { to } 21 \\
254 \cdot 9)\end{array}$ & $\begin{array}{c}18298 \cdot 8 \\
(15153 \cdot 9 \\
\text { to } 22 \\
824 \cdot 3)\end{array}$ & $7 \cdot 3(-6 \cdot 6$ to $23 \cdot 5)$ & $\begin{array}{c}309 \cdot 3 \\
(259 \cdot 6 \text { to } \\
383 \cdot 7)\end{array}$ & $\begin{array}{c}279.3 \\
(231.5 \text { to } \\
346 \cdot 9)\end{array}$ & $-9 \cdot 7(-21 \cdot 1$ to $3 \cdot 6)$ \\
\hline Chronic respiratory diseases & $\begin{array}{c}104250 \cdot 7 \\
(92540 \cdot 7 \\
\text { to } 118 \\
201 \cdot 1)\end{array}$ & $\begin{array}{c}112710 \cdot 7 \\
(98871 \cdot 9 \\
\text { to } 128 \\
147 \cdot 8)\end{array}$ & $8 \cdot 1(2 \cdot 7 \text { to } 13 \cdot 8)^{*}$ & $\begin{array}{c}1935 \cdot 3 \\
(1734 \cdot 0 \\
\text { to } \\
2180 \cdot 3)\end{array}$ & $\begin{array}{c}1754 \cdot 3 \\
(1550 \cdot 4 \\
\text { to } \\
1981 \cdot 3)\end{array}$ & $-9 \cdot 3(-13.9 \text { to }-4 \cdot 6)^{*}$ \\
\hline $\begin{array}{l}\text { Chronic obstructive pulmonary } \\
\text { disease }\end{array}$ & $\begin{array}{c}66478 \cdot 5 \\
(58577 \cdot 5 \\
\text { to } 75 \\
309 \cdot 8)\end{array}$ & $\begin{array}{c}71900 \cdot 7 \\
(61998 \cdot 5 \\
\text { to } 82 \\
621.4)\end{array}$ & $8 \cdot 2(3.0 \text { to } 13 \cdot 6)^{*}$ & $\begin{array}{c}1276 \cdot 2 \\
(1136 \cdot 1 \\
\text { to } \\
1435 \cdot 7)\end{array}$ & $\begin{array}{c}1137.9 \\
(990 \cdot 5 \text { to } \\
1299 \cdot 5)\end{array}$ & $\begin{array}{c}-10 \cdot 8(-15 \cdot 1 \text { to } \\
-6 \cdot 3)^{*}\end{array}$ \\
\hline Pneumoconiosis & $\begin{array}{c}4770 \cdot 7 \\
(3830 \cdot 8 \text { to } \\
6066 \cdot 8)\end{array}$ & $\begin{array}{c}5468 \cdot 0 \\
(4285 \cdot 5 \text { to } \\
6974 \cdot 4)\end{array}$ & $14 \cdot 4(-1 \cdot 8$ to $33 \cdot 7)$ & $\begin{array}{c}90 \cdot 3 \\
(72 \cdot 5 \text { to } \\
114 \cdot 6)\end{array}$ & $\begin{array}{c}85 \cdot 5 \\
(67 \cdot 0 \text { to } \\
108 \cdot 8)\end{array}$ & $-5 \cdot 6(-18 \cdot 9$ to $10 \cdot 3)$ \\
\hline Silicosis & $\begin{array}{c}926 \cdot 8 \\
(635 \cdot 0 \text { to } \\
1323 \cdot 0)\end{array}$ & $\begin{array}{c}983 \cdot 6 \\
(682 \cdot 7 \text { to } \\
1386 \cdot 8)\end{array}$ & $6 \cdot 0(-8 \cdot 0$ to $22 \cdot 3)$ & $\begin{array}{c}17 \cdot 5 \\
(12 \cdot 0 \text { to } \\
24 \cdot 9)\end{array}$ & $\begin{array}{c}15 \cdot 4 \\
(10 \cdot 8 \text { to } \\
21 \cdot 5)\end{array}$ & $-12 \cdot 2(-23 \cdot 6$ to $1 \cdot 2)$ \\
\hline Asbestosis & $\begin{array}{c}467.4 \\
(340 \cdot 7 \text { to } \\
629 \cdot 7)\end{array}$ & $\begin{array}{c}554 \cdot 3 \\
(403 \cdot 8 \text { to } \\
754.9)\end{array}$ & $18 \cdot 2(1.8 \text { to } 40 \cdot 0)^{*}$ & $\begin{array}{l}8 \cdot 7(6 \cdot 3 \\
\text { to } 11 \cdot 7)\end{array}$ & $\begin{array}{l}8 \cdot 5(6 \cdot 2 \\
\text { to } 11 \cdot 6)\end{array}$ & $-2 \cdot 2(-15 \cdot 9$ to $15 \cdot 7)$ \\
\hline Coal workers' pneumoconiosis & $\begin{array}{c}531 \cdot 1 \\
(393 \cdot 2 \text { to } \\
749 \cdot 6)\end{array}$ & $\begin{array}{c}600 \cdot 2 \\
(447 \cdot 6 \text { to } \\
838 \cdot 6)\end{array}$ & $13 \cdot 0(-2 \cdot 5$ to $31 \cdot 4)$ & $\begin{array}{l}9 \cdot 8(7 \cdot 3 \\
\text { to } 14 \cdot 0)\end{array}$ & $\begin{array}{l}9 \cdot 2(6 \cdot 9 \\
\text { to } 12 \cdot 8)\end{array}$ & $-6 \cdot 8(-19 \cdot 3$ to $8 \cdot 2)$ \\
\hline Other pneumoconiosis & $\begin{array}{c}2845 \cdot 4 \\
(2258 \cdot 8 \text { to } \\
3640 \cdot 8)\end{array}$ & $\begin{array}{c}3329 \cdot 9 \\
(2525 \cdot 9 \text { to } \\
4296 \cdot 4)\end{array}$ & $16 \cdot 7(-3 \cdot 9$ to $40 \cdot 9)$ & $\begin{array}{c}54 \cdot 3 \\
(43 \cdot 2 \text { to } \\
69 \cdot 6)\end{array}$ & $\begin{array}{c}52.4 \\
(39 \cdot 8 \text { to } \\
67 \cdot 9)\end{array}$ & $-3 \cdot 7(-20 \cdot 4$ to $16 \cdot 5)$ \\
\hline Asthma & $\begin{array}{c}22240 \cdot 4 \\
(17995 \cdot 5 \\
\text { to } 27 \\
896 \cdot 9)\end{array}$ & $\begin{array}{c}22182 \cdot 7 \\
(17852 \cdot 3 \\
\text { to } 28 \\
053 \cdot 6)\end{array}$ & $-0 \cdot 5(-6 \cdot 7$ to $6 \cdot 9)$ & $\begin{array}{c}373 \cdot 1 \\
(304 \cdot 1 \text { to } \\
471.9)\end{array}$ & $\begin{array}{c}326 \cdot 4 \\
(263 \cdot 3 \text { to } \\
414 \cdot 3)\end{array}$ & $\begin{array}{c}-12 \cdot 7(-19 \cdot 0 \text { to } \\
-6 \cdot 1)^{*}\end{array}$ \\
\hline $\begin{array}{l}\text { Interstitial lung disease and } \\
\text { pulmonary sarcoidosis }\end{array}$ & $\begin{array}{c}5929 \cdot 3 \\
(4466 \cdot 5 \text { to } \\
7311 \cdot 7)\end{array}$ & $\begin{array}{c}8178 \cdot 0 \\
(6359 \cdot 8 \text { to } \\
10399 \cdot 7)\end{array}$ & $38.8(18 \cdot 3$ to $56 \cdot 6) *$ & $\begin{array}{l}116 \cdot 0 \\
(88 \cdot 1 \text { to } \\
142 \cdot 5)\end{array}$ & $\begin{array}{c}131 \cdot 3 \\
(102 \cdot 3 \text { to } \\
166 \cdot 8)\end{array}$ & $13 \cdot 8(-2 \cdot 7$ to $28 \cdot 6)$ \\
\hline Other chronic respiratory diseases & $\begin{array}{c}4831 \cdot 8 \\
(3897 \cdot 3 \text { to } \\
5955 \cdot 2)\end{array}$ & $\begin{array}{c}4981 \cdot 3 \\
(4025 \cdot 8 \text { to } \\
6185 \cdot 0)\end{array}$ & $2 \cdot 9(-5 \cdot 0$ to $11 \cdot 6)$ & $\begin{array}{c}79 \cdot 6 \\
(63 \cdot 8 \text { to } \\
99 \cdot 0)\end{array}$ & $\begin{array}{c}73 \cdot 2 \\
(59 \cdot 3 \text { to } \\
90 \cdot 8)\end{array}$ & $-8 \cdot 3(-15 \cdot 0 \text { to }-0 \cdot 6)^{*}$ \\
\hline Cirrhosis & $\begin{array}{c}35528 \cdot 4 \\
(34221 \cdot 3 \\
\text { to } 36 \\
967 \cdot 4)\end{array}$ & $\begin{array}{c}36858 \cdot 1 \\
(35053 \cdot 9 \\
\text { to } 39 \\
022 \cdot 5)\end{array}$ & $3 \cdot 6(-1 \cdot 5$ to $9 \cdot 7)$ & $\begin{array}{c}606 \cdot 6 \\
(585 \cdot 5 \text { to } \\
629 \cdot 5)\end{array}$ & $\begin{array}{c}535 \cdot 9 \\
(510 \cdot 2 \text { to } \\
567 \cdot 0)\end{array}$ & $\begin{array}{c}-11 \cdot 8(-16 \cdot 1 \text { to } \\
-6 \cdot 8)^{*}\end{array}$ \\
\hline Cirrhosis due to hepatitis B & $\begin{array}{c}9321 \cdot 9 \\
(8709 \cdot 4 \text { to } \\
9936 \cdot 2)\end{array}$ & $\begin{array}{c}9399 \cdot 4 \\
(8557 \cdot 4 \text { to } \\
10303 \cdot 7)\end{array}$ & $0 \cdot 7(-8 \cdot 4$ to $12 \cdot 2)$ & $\begin{array}{c}159 \cdot 9 \\
(149 \cdot 6 \text { to } \\
170 \cdot 4)\end{array}$ & $\begin{array}{c}136 \cdot 9 \\
(124 \cdot 9 \text { to } \\
149 \cdot 7)\end{array}$ & $\begin{array}{c}-14 \cdot 5(-22 \cdot 0 \text { to } \\
-4 \cdot 7)^{*}\end{array}$ \\
\hline Cirrhosis due to hepatitis C & $\begin{array}{c}8937 \cdot 8 \\
(8404 \cdot 8 \text { to } \\
9505 \cdot 2)\end{array}$ & $\begin{array}{c}9939 \cdot 9 \\
(9200 \cdot 4 \text { to } \\
10788 \cdot 7)\end{array}$ & $11.3(1.7 \text { to } 22.5)^{*}$ & $\begin{array}{c}156 \cdot 3 \\
(147 \cdot 2 \text { to } \\
165 \cdot 7)\end{array}$ & $\begin{array}{c}146 \cdot 2 \\
(135 \cdot 9 \text { to } \\
158 \cdot 6)\end{array}$ & $-6 \cdot 5(-14 \cdot 2$ to $2 \cdot 6)$ \\
\hline Cirrhosis due to alcohol use & $\begin{array}{c}11182 \cdot 1 \\
(10401 \cdot 0 \\
\text { to } 11 \\
948 \cdot 2)\end{array}$ & $\begin{array}{l}10886 \cdot 3 \\
(9929 \cdot 1 \text { to } \\
11927 \cdot 3)\end{array}$ & $-2 \cdot 8(-12 \cdot 5$ to $8 \cdot 9)$ & $\begin{array}{c}195 \cdot 0 \\
(181 \cdot 9 \text { to } \\
207 \cdot 7)\end{array}$ & $\begin{array}{c}159 \cdot 7 \\
(146 \cdot 2 \text { to } \\
174 \cdot 5)\end{array}$ & $\begin{array}{c}-18 \cdot 3(-26 \cdot 0 \text { to } \\
-8 \cdot 6)^{*}\end{array}$ \\
\hline
\end{tabular}




\begin{tabular}{|c|c|c|c|c|c|c|}
\hline & \multicolumn{3}{|c|}{ All ages DALYs (thousands) ${ }^{*}$} & \multicolumn{3}{|c|}{ Age-standardised DALYs (per 100000 ) } \\
\hline & 2005 & 2013 & Percentage change & 2005 & 2013 & Percentage change \\
\hline Cirrhosis due to other causes & $\begin{array}{c}6086 \cdot 6 \\
(5445 \cdot 6 \text { to } \\
6855 \cdot 8)\end{array}$ & $\begin{array}{c}6632 \cdot 4 \\
(5969 \cdot 0 \text { to } \\
7450 \cdot 4)\end{array}$ & $9 \cdot 0(-6 \cdot 4$ to $26 \cdot 6)$ & $\begin{array}{c}95 \cdot 3 \\
(85 \cdot 3 \text { to } \\
107 \cdot 3)\end{array}$ & $\begin{array}{c}93 \cdot 1 \\
(83 \cdot 9 \text { to } \\
104 \cdot 3)\end{array}$ & $-2 \cdot 4(-16 \cdot 2$ to $13 \cdot 9)$ \\
\hline Digestive diseases & $\begin{array}{c}37037 \cdot 5 \\
(33945 \cdot 1 \\
\text { to } 40 \\
627 \cdot 4)\end{array}$ & $\begin{array}{c}37341 \cdot 2 \\
(33670 \cdot 4 \\
\text { to } 41 \\
452 \cdot 4)\end{array}$ & $0 \cdot 7(-5 \cdot 3$ to $7 \cdot 7)$ & $\begin{array}{l}643 \cdot 0 \\
(589 \cdot 7 \text { to } \\
704 \cdot 5)\end{array}$ & $\begin{array}{l}557 \cdot 3 \\
(502 \cdot 6 \text { to } \\
617 \cdot 9)\end{array}$ & $\begin{array}{c}-13 \cdot 5(-18 \cdot 2 \text { to } \\
-7 \cdot 7)^{*}\end{array}$ \\
\hline Peptic ulcer disease & $\begin{array}{c}9090 \cdot 0 \\
(7900 \cdot 7 \text { to } \\
10341 \cdot 3)\end{array}$ & $\begin{array}{l}8457 \cdot 8 \\
(6967 \cdot 0 \text { to } \\
9805 \cdot 3)\end{array}$ & $-7 \cdot 2(-15 \cdot 3$ to $2 \cdot 8)$ & $\begin{array}{c}163 \cdot 6 \\
(142 \cdot 5 \text { to } \\
185 \cdot 8)\end{array}$ & $\begin{array}{c}128 \cdot 5 \\
(106 \cdot 4 \text { to } \\
148 \cdot 5)\end{array}$ & $\begin{array}{c}-21.7(-27.9 \text { to } \\
-13.7)\end{array}$ \\
\hline Gastritis and duodenitis & $\begin{array}{c}3900 \cdot 1 \\
(2949 \cdot 8 \text { to } \\
5033 \cdot 5)\end{array}$ & $\begin{array}{c}3860 \cdot 1 \\
(2931 \cdot 3 \text { to } \\
4985 \cdot 7)\end{array}$ & $-1.0(-6 \cdot 7$ to 4.7$)$ & $\begin{array}{l}67 \cdot 1 \\
(51 \cdot 1 \text { to } \\
86 \cdot 6)\end{array}$ & $\begin{array}{c}58 \cdot 1 \\
(44 \cdot 1 \text { to } \\
75 \cdot 2)\end{array}$ & $\begin{array}{c}-13 \cdot 5(-18 \cdot 2 \text { to } \\
-8 \cdot 7)^{*}\end{array}$ \\
\hline Appendicitis & $\begin{array}{c}3082 \cdot 0 \\
(2452 \cdot 4 \text { to } \\
3652 \cdot 8)\end{array}$ & $\begin{array}{c}2760 \cdot 7 \\
(2084 \cdot 3 \text { to } \\
3383 \cdot 6)\end{array}$ & $-10 \cdot 9(-23 \cdot 0$ to $4 \cdot 8)$ & $\begin{array}{c}48 \cdot 7 \\
(38 \cdot 6 \text { to } \\
57 \cdot 5)\end{array}$ & $\begin{array}{c}39 \cdot 1 \\
(29 \cdot 4 \text { to } \\
47 \cdot 8)\end{array}$ & $\begin{array}{c}-20 \cdot 0(-30 \cdot 4 \text { to } \\
-6 \cdot 4)^{*}\end{array}$ \\
\hline $\begin{array}{l}\text { Paralytic ileus and intestinal } \\
\text { obstruction }\end{array}$ & $\begin{array}{c}5468 \cdot 0 \\
(4182 \cdot 4 \text { to } \\
7560 \cdot 0)\end{array}$ & $\begin{array}{c}6071 \cdot 7 \\
(4684 \cdot 6 \text { to } \\
8303 \cdot 2)\end{array}$ & $10.9(1.4 \text { to } 22.2)^{*}$ & $\begin{array}{c}93 \cdot 8 \\
(71 \cdot 4 \text { to } \\
129 \cdot 9)\end{array}$ & $\begin{array}{c}90 \cdot 0 \\
(69 \cdot 3 \text { to } \\
123 \cdot 2)\end{array}$ & $-4 \cdot 2(-12 \cdot 2$ to $5 \cdot 1)$ \\
\hline $\begin{array}{l}\text { Inguinal, femoral, and abdominal } \\
\text { hernia }\end{array}$ & $\begin{array}{l}982 \cdot 8 \\
(773 \cdot 4 \text { to } \\
1426 \cdot 7)\end{array}$ & $\begin{array}{l}954.8 \\
(742.2 \text { to } \\
1381 \cdot 4)\end{array}$ & $-2 \cdot 0(-16 \cdot 9$ to $9 \cdot 4)$ & $\begin{array}{l}17 \cdot 8 \\
(14 \cdot 1 \text { to } \\
25 \cdot 4)\end{array}$ & $\begin{array}{l}14.7 \\
(11.5 \text { to } \\
21 \cdot 2)\end{array}$ & $\begin{array}{c}-16 \cdot 5(-28 \cdot 2 \text { to } \\
-6 \cdot 2)^{*}\end{array}$ \\
\hline Inflammatory bowel disease & $\begin{array}{c}3545 \cdot 7 \\
(2854 \cdot 3 \text { to } \\
4368 \cdot 0)\end{array}$ & $\begin{array}{c}3729 \cdot 1 \\
(2964 \cdot 8 \text { to } \\
4665 \cdot 3)\end{array}$ & $5 \cdot 2(0 \cdot 2 \text { to } 10 \cdot 0)^{*}$ & $\begin{array}{c}59 \cdot 1 \\
(47 \cdot 4 \text { to } \\
72 \cdot 8)\end{array}$ & $\begin{array}{c}54 \cdot 1 \\
(43 \cdot 1 \text { to } \\
67 \cdot 6)\end{array}$ & $-8.6(-12.6 \text { to }-4 \cdot 7)^{*}$ \\
\hline Vascular intestinal disorders & $\begin{array}{c}1158 \cdot 5 \\
(729 \cdot 7 \text { to } \\
1739 \cdot 6)\end{array}$ & $\begin{array}{l}1241 \cdot 5 \\
(800 \cdot 0 \text { to } \\
1839 \cdot 5)\end{array}$ & $7 \cdot 2(-0.5$ to 14.9$)$ & $\begin{array}{c}22 \cdot 6 \\
(14 \cdot 3 \text { to } \\
33 \cdot 4)\end{array}$ & $\begin{array}{l}20 \cdot 0 \\
(13 \cdot 0 \text { to } \\
29 \cdot 4)\end{array}$ & $\begin{array}{c}-11 \cdot 6(-17 \cdot 8 \text { to } \\
-5 \cdot 5)^{*}\end{array}$ \\
\hline Gallbladder and biliary diseases & $\begin{array}{c}2420 \cdot 2 \\
(2141 \cdot 2 \text { to } \\
2789 \cdot 6)\end{array}$ & $\begin{array}{c}2559 \cdot 7 \\
(2191 \cdot 8 \text { to } \\
2924 \cdot 8)\end{array}$ & $6 \cdot 0(-0.8$ to $11 \cdot 1)$ & $\begin{array}{l}44 \cdot 6 \\
(39 \cdot 6 \text { to } \\
51 \cdot 3)\end{array}$ & $\begin{array}{c}39 \cdot 7 \\
(34 \cdot 0 \text { to } \\
45 \cdot 5)\end{array}$ & $\begin{array}{c}-10 \cdot 8(-16 \cdot 5 \text { to } \\
-6 \cdot 8)^{*}\end{array}$ \\
\hline Pancreatitis & $\begin{array}{c}3925 \cdot 9 \\
(2838 \cdot 2 \text { to } \\
4834 \cdot 3)\end{array}$ & $\begin{array}{c}4198 \cdot 8 \\
(3062 \cdot 6 \text { to } \\
5140 \cdot 9)\end{array}$ & $7 \cdot 0(-2 \cdot 3$ to $17 \cdot 5)$ & $\begin{array}{c}66 \cdot 0 \\
(47 \cdot 7 \text { to } \\
81 \cdot 2)\end{array}$ & $\begin{array}{l}60 \cdot 8 \\
(44 \cdot 4 \text { to } \\
74 \cdot 4)\end{array}$ & $-7.8(-15.5$ to 0.9$)$ \\
\hline Other digestive diseases & $\begin{array}{c}3464 \cdot 3 \\
(2944 \cdot 0 \text { to } \\
4039 \cdot 4)\end{array}$ & $\begin{array}{c}3506 \cdot 9 \\
(2924 \cdot 0 \text { to } \\
4110 \cdot 0)\end{array}$ & $1.2(-3.9$ to $7 \cdot 0)$ & $\begin{array}{c}59 \cdot 8 \\
(51 \cdot 0 \text { to } \\
69 \cdot 8)\end{array}$ & $\begin{array}{c}52 \cdot 4 \\
(43 \cdot 8 \text { to } \\
61 \cdot 3)\end{array}$ & $\begin{array}{c}-12 \cdot 5(-16 \cdot 6 \text { to } \\
-7 \cdot 7)^{*}\end{array}$ \\
\hline Neurological disorders & $\begin{array}{c}72438 \cdot 0 \\
(56404 \cdot 6 \\
\text { to } 91 \\
027 \cdot 3)\end{array}$ & $\begin{array}{c}84048 \cdot 0 \\
(65694 \cdot 2 \\
\text { to } 105 \\
692 \cdot 5)\end{array}$ & $16.1(13.4 \text { to } 18.4)^{*}$ & $\begin{array}{c}1267 \cdot 8 \\
(1007 \cdot 2 \\
\text { to } \\
1568 \cdot 2)\end{array}$ & $\begin{array}{c}1264 \cdot 4 \\
(1000 \cdot 7 \\
\text { to } \\
1571 \cdot 9)\end{array}$ & $-0 \cdot 2(-2 \cdot 5$ to $1 \cdot 7)$ \\
\hline $\begin{array}{l}\text { Alzheimer's disease and other } \\
\text { dementias }\end{array}$ & $\begin{array}{c}17737 \cdot 9 \\
(16089 \cdot 2 \\
\text { to } 19 \\
551.9)\end{array}$ & $\begin{array}{c}22238 \cdot 9 \\
(19993 \cdot 3 \\
\text { to } 24 \\
542 \cdot 5)\end{array}$ & $25.3(21.2 \text { to } 29.7)^{*}$ & $\begin{array}{c}404 \cdot 3 \\
(366 \cdot 7 \text { to } \\
445 \cdot 2)\end{array}$ & $\begin{array}{c}394.6 \\
(354.9 \text { to } \\
435 \cdot 5)\end{array}$ & $-2 \cdot 4(-5 \cdot 6$ to $1 \cdot 1)$ \\
\hline Parkinson's disease & $\begin{array}{c}1489 \cdot 6 \\
(1240 \cdot 4 \text { to } \\
1727 \cdot 5)\end{array}$ & $\begin{array}{c}1829 \cdot 0 \\
(1502 \cdot 7 \text { to } \\
2135 \cdot 0)\end{array}$ & $22 \cdot 9(18 \cdot 0 \text { to } 26 \cdot 8)^{*}$ & $\begin{array}{c}31 \cdot 5 \\
(26 \cdot 2 \text { to } \\
36 \cdot 4)\end{array}$ & $\begin{array}{c}31 \cdot 2 \\
(25 \cdot 7 \text { to } \\
36 \cdot 3)\end{array}$ & $-0 \cdot 8(-4 \cdot 8$ to $2 \cdot 6)$ \\
\hline Epilepsy & $\begin{array}{c}13039.4 \\
(10714.7 \\
\text { to } 15 \\
492 \cdot 8)\end{array}$ & $\begin{array}{c}13372 \cdot 1 \\
(10920 \cdot 9 \\
\text { to } 15 \\
979 \cdot 4)\end{array}$ & $2 \cdot 4(-4 \cdot 5$ to $10 \cdot 0)$ & $\begin{array}{c}196 \cdot 8 \\
(161 \cdot 5 \text { to } \\
233.9)\end{array}$ & $\begin{array}{c}185 \cdot 2 \\
(151 \cdot 2 \text { to } \\
221 \cdot 3)\end{array}$ & $-6 \cdot 0(-12 \cdot 3$ to $0 \cdot 9)$ \\
\hline Multiple sclerosis & $\begin{array}{c}1150 \cdot 9 \\
(906 \cdot 8 \text { to } \\
1361 \cdot 7)\end{array}$ & $\begin{array}{c}1342 \cdot 8 \\
(1068 \cdot 4 \text { to } \\
1625 \cdot 8)\end{array}$ & $16 \cdot 6(9 \cdot 3 \text { to } 25 \cdot 1)^{*}$ & $\begin{array}{c}19 \cdot 3 \\
(15 \cdot 2 \text { to } \\
22 \cdot 9)\end{array}$ & $\begin{array}{c}19 \cdot 2 \\
(15 \cdot 3 \text { to } \\
23 \cdot 1)\end{array}$ & $-0 \cdot 8(-7 \cdot 2$ to $6 \cdot 4)$ \\
\hline
\end{tabular}




\begin{tabular}{|c|c|c|c|c|c|c|}
\hline & \multicolumn{3}{|c|}{ All ages DALYs (thousands) ${ }^{*}$} & \multicolumn{3}{|c|}{$\underline{\text { Age-standardised DALYs (per } 100000)^{*}}$} \\
\hline & 2005 & 2013 & Percentage change & 2005 & 2013 & Percentage change \\
\hline Migraine & $\begin{array}{c}25780 \cdot 9 \\
(15613 \cdot 2 \\
\text { to } 37 \\
987 \cdot 5)\end{array}$ & $\begin{array}{c}28898 \cdot 1 \\
(17585 \cdot 8 \\
\text { to } 42 \\
420 \cdot 1)\end{array}$ & $12 \cdot 1(8 \cdot 8 \text { to } 15 \cdot 3)^{*}$ & $\begin{array}{c}395 \cdot 8 \\
(240 \cdot 2 \text { to } \\
582 \cdot 7)\end{array}$ & $\begin{array}{c}398 \cdot 4 \\
(242.4 \text { to } \\
584.9)\end{array}$ & $0 \cdot 6(-2 \cdot 2$ to $3 \cdot 5$ \\
\hline Tension-type headache & $\begin{array}{c}2031 \cdot 8 \\
(985.5 \text { to } \\
3558 \cdot 8)\end{array}$ & $\begin{array}{c}2363 \cdot 2 \\
(1151.9 \text { to } \\
4155 \cdot 0)\end{array}$ & $16.3(13.7 \text { to } 19.0)^{*}$ & $\begin{array}{l}31 \cdot 6 \\
(15 \cdot 3 \text { to } \\
55 \cdot 2)\end{array}$ & $\begin{array}{l}32 \cdot 9 \\
(16 \cdot 0 \text { to } \\
57 \cdot 8)\end{array}$ & $4.2(1.9 \text { to } 6.6)^{*}$ \\
\hline Medication overuse headache & $\begin{array}{c}7577 \cdot 4 \\
(4426 \cdot 0 \text { to } \\
11589.5)\end{array}$ & $\begin{array}{c}9845 \cdot 7 \\
(5777.9 \text { to } \\
15100 \cdot 3)\end{array}$ & $30.0(22.7 \text { to } 37.1)^{*}$ & $\begin{array}{c}121 \cdot 8 \\
(71 \cdot 3 \text { to } \\
186 \cdot 3)\end{array}$ & $\begin{array}{l}138.5 \\
(81 \cdot 3 \text { to } \\
212 \cdot 1)\end{array}$ & $13.7(7.3 \text { to } 19.8)^{*}$ \\
\hline Other neurological disorders & $\begin{array}{c}3630 \cdot 1 \\
(3079 \cdot 2 \text { to } \\
4245 \cdot 0)\end{array}$ & $\begin{array}{c}4158 \cdot 2 \\
(3465 \cdot 2 \text { to } \\
4719 \cdot 9)\end{array}$ & $15 \cdot 0(7 \cdot 2 \text { to } 20 \cdot 4)^{*}$ & $\begin{array}{l}66 \cdot 7 \\
(56 \cdot 2 \text { to } \\
78 \cdot 3)\end{array}$ & $\begin{array}{l}64 \cdot 4 \\
(53 \cdot 8 \text { to } \\
73 \cdot 5)\end{array}$ & $-3 \cdot 0(-9 \cdot 2$ to $1 \cdot 5)$ \\
\hline $\begin{array}{l}\text { Mental and substance use } \\
\text { disorders }\end{array}$ & $\begin{array}{c}157899 \cdot 7 \\
(117 \\
039 \cdot 4 \text { to } \\
202 \\
585 \cdot 3)\end{array}$ & $\begin{array}{c}173177 \cdot 4 \\
(127 \\
426 \cdot 5 \text { to } \\
221 \\
734 \cdot 1)\end{array}$ & $9 \cdot 7(7.8 \text { to } 11 \cdot 0)^{*}$ & \begin{tabular}{|c|}
$2443 \cdot 3$ \\
$(1812 \cdot 5$ \\
to \\
$3131 \cdot 3)$
\end{tabular} & $\begin{array}{c}2399 \cdot 8 \\
(1765 \cdot 2 \\
\text { to } \\
3064 \cdot 4)\end{array}$ & $-1 \cdot 8(-3.4 \text { to }-0 \cdot 7)^{*}$ \\
\hline Schizophrenia & $\begin{array}{c}13972 \cdot 2 \\
(10445 \cdot 2 \\
\text { to } 16 \\
601 \cdot 6)\end{array}$ & $\begin{array}{c}15687 \cdot 2 \\
(11647 \cdot 4 \\
\text { to } 18 \\
704 \cdot 1)\end{array}$ & $12 \cdot 3(10 \cdot 8 \text { to } 13 \cdot 6)^{*}$ & $\begin{array}{c}221 \cdot 6 \\
(165 \cdot 7 \text { to } \\
263 \cdot 0)\end{array}$ & $\begin{array}{c}217.2 \\
(161.4 \text { to } \\
258 \cdot 7)\end{array}$ & $-1.9(-3 \cdot 2 \text { to }-0.8)^{*}$ \\
\hline Alcohol use disorders & $\begin{array}{c}13856 \cdot 3 \\
(10676 \cdot 5 \\
\text { to } 17 \\
518 \cdot 8)\end{array}$ & $\begin{array}{l}12772 \cdot 1 \\
(9872 \cdot 5 \text { to } \\
16401 \cdot 5)\end{array}$ & $-8 \cdot 0(-11.5 \text { to }-3 \cdot 5)^{*}$ & $\begin{array}{c}217 \cdot 5 \\
(168 \cdot 0 \text { to } \\
274 \cdot 0)\end{array}$ & $\begin{array}{c}175 \cdot 7 \\
(136 \cdot 1 \text { to } \\
224 \cdot 8)\end{array}$ & $\begin{array}{c}-19 \cdot 3(-22 \cdot 7 \text { to } \\
-15 \cdot 0)^{*}\end{array}$ \\
\hline Drug use disorders & $\begin{array}{c}16573 \cdot 6 \\
(12990 \cdot 6 \\
\text { to } 20 \\
087 \cdot 4)\end{array}$ & $\begin{array}{c}17953 \cdot 0 \\
(14163 \cdot 9 \\
\text { to } 21 \\
969 \cdot 4)\end{array}$ & $8 \cdot 3(4 \cdot 5$ to $11 \cdot 8)$ & $\begin{array}{c}247 \cdot 3 \\
(194 \cdot 2 \text { to } \\
299 \cdot 1)\end{array}$ & $\begin{array}{l}242 \cdot 2 \\
(191 \cdot 3 \text { to } \\
296 \cdot 2)\end{array}$ & $-2 \cdot 1(-5 \cdot 5$ to $1 \cdot 0)$ \\
\hline Opioid use disorders & $\begin{array}{c}8577 \cdot 2 \\
(6762 \cdot 8 \text { to } \\
10512 \cdot 9)\end{array}$ & $\begin{array}{c}8136 \cdot 2 \\
(6171 \cdot 1 \text { to } \\
10485 \cdot 5)\end{array}$ & $-5 \cdot 4(-11 \cdot 2$ to $0 \cdot 9)$ & $\begin{array}{c}130 \cdot 1 \\
(102 \cdot 8 \text { to } \\
159 \cdot 0)\end{array}$ & $\begin{array}{c}110 \cdot 3 \\
(83 \cdot 7 \text { to } \\
142 \cdot 2)\end{array}$ & $\begin{array}{c}-15 \cdot 4(-20 \cdot 6 \text { to } \\
-9 \cdot 6)^{*}\end{array}$ \\
\hline Cocaine use disorders & $\begin{array}{c}1056 \cdot 2 \\
(739 \cdot 6 \text { to } \\
1439 \cdot 9)\end{array}$ & $\begin{array}{c}1200 \cdot 4 \\
(851 \cdot 2 \text { to } \\
1619 \cdot 0)\end{array}$ & $13.8(9 \cdot 3 \text { to } 18 \cdot 5)^{*}$ & $\begin{array}{l}15 \cdot 6 \\
(11 \cdot 0 \text { to } \\
21 \cdot 3)\end{array}$ & $\begin{array}{l}16 \cdot 1 \\
(11 \cdot 5 \text { to } \\
21 \cdot 7)\end{array}$ & $3 \cdot 3(-0 \cdot 8$ to $7 \cdot 5)$ \\
\hline Amphetamine use disorders & $\begin{array}{c}1937.0 \\
(1244.8 \text { to } \\
2768 \cdot 9)\end{array}$ & $\begin{array}{l}2117 \cdot 2 \\
(1388 \cdot 2 \text { to } \\
2987 \cdot 5)\end{array}$ & $9 \cdot 3(4.9 \text { to } 14.3)^{*}$ & $\begin{array}{l}27.9 \\
(18 \cdot 0 \text { to } \\
39 \cdot 9)\end{array}$ & $\begin{array}{l}28 \cdot 2 \\
(18 \cdot 5 \text { to } \\
39 \cdot 8)\end{array}$ & $1 \cdot 1(-2 \cdot 9$ to $5 \cdot 5)$ \\
\hline Cannabis use disorders & $\begin{array}{c}383.5 \\
(254.8 \text { to } \\
557 \cdot 0)\end{array}$ & $\begin{array}{c}395.6 \\
(261.2 \text { to } \\
576.2)\end{array}$ & $3 \cdot 2(0 \cdot 1 \text { to } 6 \cdot 4)^{*}$ & $\begin{array}{l}5.5(3.6 \\
\text { to } 7 \cdot 9)\end{array}$ & $\begin{array}{l}5 \cdot 3(3 \cdot 5 \\
\text { to } 7 \cdot 7)\end{array}$ & $-3 \cdot 0(-5 \cdot 8 \text { to }-0 \cdot 1)^{*}$ \\
\hline Other drug use disorders & $\begin{array}{c}4619 \cdot 7 \\
(3665 \cdot 7 \text { to } \\
5670 \cdot 2)\end{array}$ & $\begin{array}{c}6103 \cdot 5 \\
(5006 \cdot 4 \text { to } \\
7312 \cdot 4)\end{array}$ & $32.3(23.5 \text { to } 41.6)^{*}$ & $\begin{array}{l}68 \cdot 1 \\
(54 \cdot 2 \text { to } \\
83 \cdot 3)\end{array}$ & $\begin{array}{l}82 \cdot 2 \\
(67.5 \text { to } \\
98 \cdot 3)\end{array}$ & $20.8(12.9 \text { to } 29.1)^{*}$ \\
\hline Depressive disorders & $\begin{array}{c}54086 \cdot 1 \\
(36401 \cdot 9 \\
\text { to } 75 \\
052 \cdot 8)\end{array}$ & $\begin{array}{c}61632 \cdot 8 \\
(41353.8 \\
\text { to } 85 \\
621.4)\end{array}$ & $14 \cdot 0(10 \cdot 4$ to $17 \cdot 1)$ & $\begin{array}{c}856 \cdot 4 \\
(580 \cdot 2 \text { to } \\
1186 \cdot 5)\end{array}$ & $\begin{array}{c}864 \cdot 4 \\
(580 \cdot 0 \text { to } \\
1202 \cdot 1)\end{array}$ & $1 \cdot 1(-2 \cdot 5$ to $3 \cdot 6)$ \\
\hline Major depressive disorder & $\begin{array}{c}45539 \cdot 4 \\
(29829 \cdot 4 \\
\text { to } 64 \\
133 \cdot 2)\end{array}$ & $\begin{array}{c}51783 \cdot 9 \\
(33888 \cdot 2 \\
\text { to } 73 \\
665 \cdot 8)\end{array}$ & $13.8(9.4 \text { to } 17 \cdot 5)^{*}$ & $\begin{array}{c}717 \cdot 2 \\
(471 \cdot 7 \text { to } \\
1011 \cdot 6)\end{array}$ & $\begin{array}{c}724 \cdot 9 \\
(475 \cdot 7 \text { to } \\
1030 \cdot 7)\end{array}$ & $1 \cdot 3(-2 \cdot 8$ to $4 \cdot 4)$ \\
\hline Dysthymia & $\begin{array}{c}8546 \cdot 7 \\
(5687 \cdot 3 \text { to } \\
12278 \cdot 3)\end{array}$ & $\begin{array}{c}9848 \cdot 9 \\
(6586 \cdot 6 \text { to } \\
14166 \cdot 0)\end{array}$ & $15 \cdot 2(14 \cdot 0 \text { to } 16 \cdot 3)^{*}$ & $\begin{array}{l}139 \cdot 3 \\
(93 \cdot 5 \text { to } \\
200 \cdot 2)\end{array}$ & $\begin{array}{l}139 \cdot 5 \\
(93 \cdot 7 \text { to } \\
200 \cdot 9)\end{array}$ & $0 \cdot 2(-0 \cdot 6$ to $0 \cdot 9)$ \\
\hline
\end{tabular}




\begin{tabular}{|c|c|c|c|c|c|c|}
\hline & \multicolumn{3}{|c|}{ All ages DALYs (thousands) ${ }^{*}$} & \multicolumn{3}{|c|}{ Age-standardised DALYs (per 100 000) } \\
\hline & 2005 & 2013 & Percentage change & 2005 & 2013 & Percentage change \\
\hline Bipolar disorder & $\begin{array}{c}8715 \cdot 9 \\
(5487 \cdot 1 \text { to } \\
13043 \cdot 4)\end{array}$ & $\begin{array}{l}9911 \cdot 1 \\
(6260 \cdot 6 \text { to } \\
14791 \cdot 0)\end{array}$ & $13.7(12.1 \text { to } 15.8)^{*}$ & $\begin{array}{l}135 \cdot 4 \\
(85 \cdot 0 \text { to } \\
201 \cdot 5)\end{array}$ & $\begin{array}{l}136 \cdot 6 \\
(86 \cdot 3 \text { to } \\
202 \cdot 5)\end{array}$ & $0.8(-0.4$ to 2.5$)$ \\
\hline Anxiety disorders & $\begin{array}{c}21949 \cdot 1 \\
(14287 \cdot 0 \\
\text { to } 31 \\
597 \cdot 3)\end{array}$ & $\begin{array}{c}24355 \cdot 8 \\
(16148 \cdot 6 \\
\text { to } 35 \\
139 \cdot 0)\end{array}$ & $11.0(8.5 \text { to } 13.6)^{*}$ & $\begin{array}{l}337.7 \\
(221.6 \text { to } \\
481.8)\end{array}$ & $\begin{array}{l}337 \cdot 7 \\
(224 \cdot 4 \text { to } \\
486 \cdot 3)\end{array}$ & $0 \cdot 0(-2 \cdot 0$ to $1 \cdot 9)$ \\
\hline Eating disorders & $\begin{array}{l}1742 \cdot 9 \\
(1135 \cdot 8 \text { to } \\
2601 \cdot 1)\end{array}$ & $\begin{array}{l}1853 \cdot 7 \\
(1189 \cdot 9 \text { to } \\
2753 \cdot 8)\end{array}$ & $6.3(4.2 \text { to } 8.5)^{*}$ & $\begin{array}{l}24 \cdot 6 \\
(16 \cdot 0 \text { to } \\
36 \cdot 8)\end{array}$ & $\begin{array}{l}24 \cdot 6 \\
(15 \cdot 9 \text { to } \\
36 \cdot 6)\end{array}$ & $0 \cdot 0(-2 \cdot 0$ to $1 \cdot 8)$ \\
\hline Anorexia nervosa & $\begin{array}{c}448 \cdot 5 \\
(302 \cdot 6 \text { to } \\
644 \cdot 7)\end{array}$ & $\begin{array}{c}474 \cdot 0 \\
(318 \cdot 2 \text { to } \\
682 \cdot 3)\end{array}$ & $5.7(1.9 \text { to } 9.4)^{*}$ & $\begin{array}{l}6 \cdot 3(4 \cdot 3 \\
\text { to } 9 \cdot 1)\end{array}$ & $\begin{array}{l}6 \cdot 3(4 \cdot 3 \\
\text { to } 9 \cdot 1)\end{array}$ & $-0 \cdot 2(-3 \cdot 8$ to $3 \cdot 2)$ \\
\hline Bulimia nervosa & $\begin{array}{c}1294 \cdot 4 \\
(797 \cdot 2 \text { to } \\
1995 \cdot 9)\end{array}$ & $\begin{array}{l}1379 \cdot 7 \\
(850 \cdot 7 \text { to } \\
2136 \cdot 6)\end{array}$ & $6.5(4 \cdot 1 \text { to } 9 \cdot 1)^{*}$ & $\begin{array}{l}18 \cdot 3 \\
(11 \cdot 3 \text { to } \\
28 \cdot 1)\end{array}$ & $\begin{array}{l}18 \cdot 3 \\
(11 \cdot 3 \text { to } \\
28 \cdot 3)\end{array}$ & $0 \cdot 0(-2 \cdot 1$ to $2 \cdot 4)$ \\
\hline Autistic spectrum disorders & $\begin{array}{c}7721 \cdot 8 \\
(5369 \cdot 6 \text { to } \\
10463 \cdot 8)\end{array}$ & $\begin{array}{c}8449 \cdot 0 \\
(5888 \cdot 1 \text { to } \\
11458 \cdot 7)\end{array}$ & $9.4(8.6 \text { to } 10 \cdot 3)^{*}$ & $\begin{array}{c}116 \cdot 6 \\
(81 \cdot 1 \text { to } \\
158 \cdot 0)\end{array}$ & $\begin{array}{c}117 \cdot 1 \\
(81 \cdot 6 \text { to } \\
158 \cdot 7)\end{array}$ & $0 \cdot 4(-0 \cdot 4$ to $1 \cdot 1)$ \\
\hline Autism & $\begin{array}{l}4884 \cdot 2 \\
(3285 \cdot 3 \text { to } \\
6671 \cdot 6)\end{array}$ & $\begin{array}{l}5345 \cdot 0 \\
(3583 \cdot 6 \text { to } \\
7309 \cdot 9)\end{array}$ & $9 \cdot 4(8 \cdot 3 \text { to } 10 \cdot 6)^{*}$ & $\begin{array}{c}73 \cdot 8 \\
(49 \cdot 6 \text { to } \\
100 \cdot 6)\end{array}$ & $\begin{array}{c}74 \cdot 1 \\
(49 \cdot 7 \text { to } \\
101 \cdot 3)\end{array}$ & $0.4(-0.6$ to 1.4$)$ \\
\hline Asperger's syndrome & $\begin{array}{l}2837.6 \\
(1981.9 \text { to } \\
3949 \cdot 6)\end{array}$ & $\begin{array}{l}3104 \cdot 0 \\
(2169 \cdot 6 \text { to } \\
4325 \cdot 0)\end{array}$ & $9.4(8.5 \text { to } 10 \cdot 3)^{*}$ & $\begin{array}{l}42 \cdot 9 \\
(30 \cdot 0 \text { to } \\
59 \cdot 6)\end{array}$ & $\begin{array}{l}43 \cdot 0 \\
(30 \cdot 1 \text { to } \\
59 \cdot 9)\end{array}$ & $0 \cdot 3(-0.5$ to $1 \cdot 1)$ \\
\hline Attention-deficit hyperactivity disc & $\begin{array}{l}\text { der } 478.9 \\
\quad(287.4 \text { to } \\
740.8)\end{array}$ & $\begin{array}{l}479 \cdot 9 \\
(287 \cdot 4 \text { to } \\
745 \cdot 8)\end{array}$ & $0 \cdot 2(-1.5$ to $1 \cdot 9)$ & $\begin{array}{l}6 \cdot 6(4 \cdot 0 \\
\text { to } 10 \cdot 2)\end{array}$ & $\begin{array}{l}6 \cdot 6(3 \cdot 9 \\
\text { to } 10 \cdot 2)\end{array}$ & $0 \cdot 0(-1 \cdot 7$ to $1 \cdot 7)$ \\
\hline Conduct disorder & $\begin{array}{l}6192 \cdot 7 \\
(3889 \cdot 5 \text { to } \\
8986 \cdot 2)\end{array}$ & $\begin{array}{l}6159 \cdot 0 \\
(3868 \cdot 2 \text { to } \\
8911 \cdot 6)\end{array}$ & $-0.5(-1.7$ to 0.6$)$ & $\begin{array}{l}84 \cdot 0 \\
(52 \cdot 7 \text { to } \\
122 \cdot 0)\end{array}$ & $\begin{array}{c}85 \cdot 3 \\
(53 \cdot 6 \text { to } \\
123 \cdot 4)\end{array}$ & $1.5(0.3 \text { to } 2.6)^{*}$ \\
\hline Idiopathic intellectual disability & $\begin{array}{c}4575 \cdot 2 \\
(3011 \cdot 1 \text { to } \\
6496 \cdot 0)\end{array}$ & $\begin{array}{c}4666 \cdot 7 \\
(3084 \cdot 8 \text { to } \\
6640 \cdot 0)\end{array}$ & $2 \cdot 1(-4 \cdot 1$ to $8 \cdot 6)$ & $\begin{array}{l}68 \cdot 0 \\
(44 \cdot 7 \text { to } \\
96 \cdot 6)\end{array}$ & $\begin{array}{l}64 \cdot 3 \\
(42 \cdot 5 \text { to } \\
91 \cdot 5)\end{array}$ & $-5 \cdot 3(-10 \cdot 9$ to $0 \cdot 7)$ \\
\hline $\begin{array}{l}\text { Other mental and substance use } \\
\text { disorders }\end{array}$ & $\begin{array}{c}8035 \cdot 0 \\
(5442 \cdot 0 \text { to } \\
10785 \cdot 3)\end{array}$ & $\begin{array}{c}9257.2 \\
(6277.9 \text { to } \\
12411.5)\end{array}$ & $15 \cdot 2(14.2 \text { to } 16 \cdot 2)^{*}$ & $\begin{array}{c}127 \cdot 6 \\
(86 \cdot 5 \text { to } \\
170 \cdot 7)\end{array}$ & $\begin{array}{c}128 \cdot 1 \\
(86 \cdot 9 \text { to } \\
171 \cdot 6)\end{array}$ & $0 \cdot 3(-0 \cdot 4$ to $1 \cdot 1$ \\
\hline $\begin{array}{l}\text { Diabetes, urogenital, blood, and } \\
\text { endocrine diseases }\end{array}$ & $\begin{array}{c}120976 \cdot 5 \\
(101 \\
712 \cdot 4 \text { to } \\
143 \\
463 \cdot 1)\end{array}$ & $\begin{array}{c}141620 \cdot 9 \\
(118 \\
713 \cdot 4 \text { to } \\
168 \\
158 \cdot 3)\end{array}$ & $17 \cdot 0(13 \cdot 1 \text { to } 21 \cdot 5)^{*}$ & $\begin{array}{c}2069 \cdot 2 \\
(1759 \cdot 6 \\
\text { to } \\
2429 \cdot 2)\end{array}$ & $\begin{array}{c}2089 \cdot 4 \\
(1759 \cdot 1 \\
\text { to } \\
2468 \cdot 6)\end{array}$ & $0 \cdot 8(-2 \cdot 1$ to $5 \cdot 2)$ \\
\hline Diabetes mellitus & $\begin{array}{c}46039.4 \\
(38599 \cdot 7 \\
\text { to } 54 \\
434.2)\end{array}$ & $\begin{array}{c}55832 \cdot 6 \\
\text { (46 374.6 } \\
\text { to } 66 \\
808.6)\end{array}$ & $21.2(17.6 \text { to } 25.3)^{*}$ & $\begin{array}{c}837.8 \\
(706 \cdot 6 \text { to } \\
981 \cdot 8)\end{array}$ & $\begin{array}{c}846 \cdot 2 \\
(704 \cdot 8 \text { to } \\
1007 \cdot 4)\end{array}$ & $0 \cdot 9(-2 \cdot 1$ to $4 \cdot 4)$ \\
\hline Acute glomerulonephritis & $\begin{array}{c}805 \cdot 7 \\
(535 \cdot 0 \text { to } \\
1067 \cdot 9)\end{array}$ & $\begin{array}{c}715 \cdot 4 \\
(519 \cdot 0 \text { to } \\
923 \cdot 1)\end{array}$ & $-11 \cdot 2(-22 \cdot 1$ to $4 \cdot 4)$ & $\begin{array}{l}12 \cdot 6(8 \cdot 5 \\
\text { to } 16 \cdot 6)\end{array}$ & $\begin{array}{l}10 \cdot 2(7 \cdot 4 \\
\text { to } 13 \cdot 1)\end{array}$ & $\begin{array}{c}-19 \cdot 4(-28 \cdot 8 \text { to } \\
-6 \cdot 2)^{*}\end{array}$ \\
\hline Chronic kidney disease & $\begin{array}{c}28349 \cdot 4 \\
(24290 \cdot 4 \\
\text { to } 31 \\
773 \cdot 7)\end{array}$ & $\begin{array}{c}33187 \cdot 2 \\
(28461 \cdot 0 \\
\text { to } 37 \\
316 \cdot 0)\end{array}$ & $17 \cdot 1(12.5 \text { to } 22 \cdot 0)^{*}$ & $\begin{array}{c}497.7 \\
(425 \cdot 7 \text { to } \\
555 \cdot 5)\end{array}$ & $\begin{array}{c}497.3 \\
(427.5 \text { to } \\
557 \cdot 2)\end{array}$ & $-0 \cdot 1(-4 \cdot 0$ to $4 \cdot 1)$ \\
\hline $\begin{array}{l}\text { Chronic kidney disease due to } \\
\text { diabetes mellitus }\end{array}$ & $\begin{array}{c}4493 \cdot 8 \\
(3673 \cdot 0 \text { to } \\
5204 \cdot 8)\end{array}$ & $\begin{array}{c}5939 \cdot 3 \\
(5014 \cdot 8 \text { to } \\
6940 \cdot 1)\end{array}$ & $31.8(25.4 \text { to } 43.0)^{*}$ & $\begin{array}{l}82.9 \\
(67.7 \text { to } \\
95.9)\end{array}$ & $\begin{array}{c}90 \cdot 9 \\
(77 \cdot 1 \text { to } \\
105 \cdot 9)\end{array}$ & $9.4(4.4 \text { to } 18.6)^{*}$ \\
\hline
\end{tabular}




\begin{tabular}{|c|c|c|c|c|c|c|}
\hline & \multicolumn{3}{|c|}{ All ages DALYs (thousands) ${ }^{*}$} & \multicolumn{3}{|c|}{ Age-standardised DALYs (per 100000 ) } \\
\hline & 2005 & 2013 & Percentage change & 2005 & 2013 & Percentage change \\
\hline $\begin{array}{l}\text { Chronic kidney disease due to } \\
\text { hypertension }\end{array}$ & $\begin{array}{c}6482 \cdot 8 \\
(5143 \cdot 8 \text { to } \\
7544 \cdot 1)\end{array}$ & $\begin{array}{c}7986.4 \\
(6335.9 \text { to } \\
9233.9)\end{array}$ & $23.1(17.4 \text { to } 29.7)^{*}$ & $\begin{array}{c}116 \cdot 3 \\
(92 \cdot 2 \text { to } \\
134 \cdot 8)\end{array}$ & $\begin{array}{c}121 \cdot 1 \\
(95 \cdot 7 \text { to } \\
139 \cdot 8)\end{array}$ & $4 \cdot 1(-0 \cdot 9$ to $9 \cdot 6)$ \\
\hline $\begin{array}{l}\text { Chronic kidney disease due to } \\
\text { glomerulonephritis }\end{array}$ & $\begin{array}{c}6585 \cdot 6 \\
(5631.4 \text { to } \\
7535 \cdot 5)\end{array}$ & $\begin{array}{c}6126 \cdot 2 \\
(5138 \cdot 3 \text { to } \\
7170 \cdot 7)\end{array}$ & $-7 \cdot 2(-12 \cdot 0 \text { to }-1 \cdot 1)^{*}$ & $\begin{array}{c}108 \cdot 1 \\
(92 \cdot 5 \text { to } \\
123 \cdot 7)\end{array}$ & $\begin{array}{c}88 \cdot 2 \\
(74 \cdot 0 \text { to } \\
103 \cdot 2)\end{array}$ & $\begin{array}{c}-18 \cdot 6(-22 \cdot 9 \text { to } \\
-13 \cdot 2)^{*}\end{array}$ \\
\hline $\begin{array}{l}\text { Chronic kidney disease due to } \\
\text { other causes }\end{array}$ & $\begin{array}{c}10787 \cdot 2 \\
(8898 \cdot 8 \text { to } \\
12207 \cdot 3)\end{array}$ & $\begin{array}{c}13135 \cdot 4 \\
(10821 \cdot 2 \\
\text { to } 14 \\
992.9)\end{array}$ & $21.7(14.7 \text { to } 28.9)^{*}$ & $\begin{array}{c}190 \cdot 4 \\
(156 \cdot 3 \text { to } \\
215 \cdot 6)\end{array}$ & $\begin{array}{c}197 \cdot 2 \\
(162 \cdot 6 \text { to } \\
225 \cdot 1)\end{array}$ & $3 \cdot 5(-2 \cdot 4$ to $9 \cdot 7)$ \\
\hline $\begin{array}{l}\text { Urinary diseases and male } \\
\text { infertility }\end{array}$ & $\begin{array}{c}8848 \cdot 5 \\
(7381 \cdot 2 \text { to } \\
10580 \cdot 7)\end{array}$ & $\begin{array}{c}10292 \cdot 4 \\
(8404 \cdot 5 \text { to } \\
12529 \cdot 3)\end{array}$ & $16 \cdot 1(12 \cdot 2 \text { to } 21 \cdot 0)^{*}$ & $\begin{array}{c}163 \cdot 6 \\
(135 \cdot 1 \text { to } \\
197 \cdot 1)\end{array}$ & $\begin{array}{c}160 \cdot 4 \\
(130 \cdot 6 \text { to } \\
196 \cdot 4)\end{array}$ & $-2 \cdot 2(-5 \cdot 2$ to $1 \cdot 6)$ \\
\hline $\begin{array}{l}\text { Interstitial nephritis and urinary } \\
\text { tract infections }\end{array}$ & $\begin{array}{c}3481.9 \\
(2922 \cdot 5 \text { to } \\
3776 \cdot 0)\end{array}$ & $\begin{array}{c}3808 \cdot 0 \\
(3143 \cdot 5 \text { to } \\
4201 \cdot 1)\end{array}$ & $9 \cdot 0(3 \cdot 4 \text { to } 16 \cdot 7)^{*}$ & $\begin{array}{c}61 \cdot 4 \\
(51 \cdot 6 \text { to } \\
66 \cdot 3)\end{array}$ & $\begin{array}{c}57 \cdot 6 \\
(47 \cdot 6 \text { to } \\
63 \cdot 3)\end{array}$ & $-6 \cdot 5(-11 \cdot 3 \text { to }-0 \cdot 2)^{*}$ \\
\hline Urolithiasis & $\begin{array}{c}923.5 \\
(699 \cdot 2 \text { to } \\
1196 \cdot 0)\end{array}$ & $\begin{array}{c}1006 \cdot 8 \\
(748 \cdot 8 \text { to } \\
1326 \cdot 3)\end{array}$ & $9.0(2.9 \text { to } 14.6)^{*}$ & $\begin{array}{c}16 \cdot 5 \\
(12 \cdot 6 \text { to } \\
21 \cdot 4)\end{array}$ & $\begin{array}{c}15 \cdot 2 \\
(11 \cdot 3 \text { to } \\
20 \cdot 0)\end{array}$ & $-7 \cdot 9(-12 \cdot 8 \text { to }-3 \cdot 4)^{*}$ \\
\hline Benign prostatic hyperplasia & $\begin{array}{c}2759 \cdot 5 \\
(1817 \cdot 4 \text { to } \\
3851 \cdot 3)\end{array}$ & $\begin{array}{c}3552.9 \\
(2316 \cdot 5 \text { to } \\
4993 \cdot 7)\end{array}$ & $28.7(25 \cdot 2 \text { to } 32 \cdot 1)^{*}$ & $\begin{array}{c}56 \cdot 9 \\
(37 \cdot 5 \text { to } \\
79 \cdot 4)\end{array}$ & $\begin{array}{c}59.1 \\
(38.6 \text { to } \\
83 \cdot 1)\end{array}$ & $3.8(1.0 \text { to } 6.6)^{*}$ \\
\hline $\begin{array}{l}\text { Male infertility due to other } \\
\text { causes }\end{array}$ & $\begin{array}{c}221 \cdot 8 \\
(95 \cdot 4 \text { to } \\
456 \cdot 2)\end{array}$ & $\begin{array}{c}258.6 \\
(111.8 \text { to } \\
531.4)\end{array}$ & $16 \cdot 4(7.7 \text { to } 26 \cdot 6)^{*}$ & $\begin{array}{l}3 \cdot 2(1 \cdot 4 \\
\text { to } 6 \cdot 7)\end{array}$ & $\begin{array}{l}3 \cdot 4(1 \cdot 5 \\
\text { to } 7 \cdot 1)\end{array}$ & $5 \cdot 6(-2 \cdot 1$ to $14 \cdot 7)$ \\
\hline Other urinary diseases & $\begin{array}{c}1461 \cdot 7 \\
(1070 \cdot 5 \text { to } \\
1708 \cdot 8)\end{array}$ & $\begin{array}{c}1666 \cdot 0 \\
(1158 \cdot 6 \text { to } \\
1976 \cdot 5)\end{array}$ & $13.9(5.7 \text { to } 23.1)^{*}$ & $\begin{array}{c}25.5 \\
(18.6 \text { to } \\
29.9)\end{array}$ & $\begin{array}{c}25.0 \\
(17 \cdot 4 \text { to } \\
29.7)\end{array}$ & $-2 \cdot 1(-8 \cdot 8$ to $5 \cdot 4)$ \\
\hline Gynaecological diseases & $\begin{array}{c}8262 \cdot 5 \\
(5405 \cdot 8 \text { to } \\
12229 \cdot 0)\end{array}$ & $\begin{array}{c}9237 \cdot 3 \\
(6081 \cdot 1 \text { to } \\
13702 \cdot 3)\end{array}$ & $11.7(8.8 \text { to } 15 \cdot 2)^{*}$ & $\begin{array}{c}124 \cdot 5 \\
(81 \cdot 5 \text { to } \\
184 \cdot 8)\end{array}$ & $\begin{array}{c}124 \cdot 5 \\
(81 \cdot 9 \text { to } \\
184 \cdot 7)\end{array}$ & $-0 \cdot 1(-2 \cdot 6$ to $3 \cdot 0)$ \\
\hline Uterine fibroids & $\begin{array}{c}2012 \cdot 5 \\
(1178 \cdot 8 \text { to } \\
3388 \cdot 1)\end{array}$ & $\begin{array}{c}2187 \cdot 3 \\
(1265 \cdot 4 \text { to } \\
3702 \cdot 5)\end{array}$ & $8.4(5.7 \text { to } 13.8)^{*}$ & $\begin{array}{c}31 \cdot 0 \\
(18 \cdot 2 \text { to } \\
52 \cdot 1)\end{array}$ & $\begin{array}{c}29 \cdot 6 \\
(17 \cdot 2 \text { to } \\
50 \cdot 2)\end{array}$ & $-4.5(-6 \cdot 9$ to $0 \cdot 2)$ \\
\hline Polycystic ovarian syndrome & $\begin{array}{c}1085 \cdot 3 \\
(512 \cdot 3 \text { to } \\
2026 \cdot 3)\end{array}$ & $\begin{array}{c}1196 \cdot 1 \\
(567 \cdot 0 \text { to } \\
2231 \cdot 8)\end{array}$ & $10.2(6.4 \text { to } 14.0)^{*}$ & $\begin{array}{l}16 \cdot 1(7 \cdot 6 \\
\text { to } 30 \cdot 0)\end{array}$ & $\begin{array}{l}16 \cdot 0(7 \cdot 6 \\
\text { to } 29 \cdot 9)\end{array}$ & $-0.5(-3.8$ to $2 \cdot 8)$ \\
\hline $\begin{array}{l}\text { Female infertility due to other } \\
\text { causes }\end{array}$ & $\begin{array}{c}169 \cdot 4 \\
(68 \cdot 0 \text { to } \\
357 \cdot 4)\end{array}$ & $\begin{array}{c}191 \cdot 9 \\
(75 \cdot 2 \text { to } \\
399 \cdot 1)\end{array}$ & $13 \cdot 2(2 \cdot 6$ to $25 \cdot 3) *$ & $\begin{array}{l}2 \cdot 5(1 \cdot 0 \\
\text { to } 5 \cdot 2)\end{array}$ & $\begin{array}{l}2 \cdot 6(1 \cdot 0 \\
\text { to } 5 \cdot 3)\end{array}$ & $4 \cdot 0(-5 \cdot 6$ to $14 \cdot 9)$ \\
\hline Endometriosis & $\begin{array}{c}1227 \cdot 9 \\
(824 \cdot 6 \text { to } \\
1673 \cdot 5)\end{array}$ & $\begin{array}{c}1371 \cdot 5 \\
(917 \cdot 7 \text { to } \\
1873 \cdot 2)\end{array}$ & $11.8(7.4 \text { to } 16 \cdot 6)^{*}$ & $\begin{array}{c}18 \cdot 4 \\
(12 \cdot 3 \text { to } \\
25 \cdot 0)\end{array}$ & $\begin{array}{c}18 \cdot 4 \\
(12 \cdot 3 \text { to } \\
25 \cdot 1)\end{array}$ & $0 \cdot 0(-3.9$ to $4 \cdot 4)$ \\
\hline Genital prolapse & $\begin{array}{c}960 \cdot 4 \\
(483 \cdot 2 \text { to } \\
1792 \cdot 7)\end{array}$ & $\begin{array}{c}1111 \cdot 1 \\
(550 \cdot 4 \text { to } \\
2058 \cdot 2)\end{array}$ & $15.5(12.4 \text { to } 19.2)^{*}$ & $\begin{array}{l}15 \cdot 5(7 \cdot 8 \\
\text { to } 28 \cdot 9)\end{array}$ & $\begin{array}{l}15 \cdot 5(7 \cdot 7 \\
\text { to } 28 \cdot 7)\end{array}$ & $-0 \cdot 6(-3 \cdot 2$ to $2 \cdot 5)$ \\
\hline Premenstrual syndrome & $\begin{array}{c}2136 \cdot 2 \\
(1333 \cdot 1 \text { to } \\
3184 \cdot 3)\end{array}$ & $\begin{array}{c}2548 \cdot 6 \\
(1581 \cdot 3 \text { to } \\
3777 \cdot 0)\end{array}$ & $19.0(9.9 \text { to } 30.9)^{*}$ & $\begin{array}{c}31 \cdot 1 \\
(19 \cdot 4 \text { to } \\
46 \cdot 3)\end{array}$ & $\begin{array}{c}33.9 \\
(21 \cdot 0 \text { to } \\
50 \cdot 2)\end{array}$ & $8.7(0.4 \text { to } 19.6)^{*}$ \\
\hline Other gynaecological diseases & $\begin{array}{c}670 \cdot 9 \\
(472 \cdot 0 \text { to } \\
922 \cdot 5)\end{array}$ & $\begin{array}{c}630 \cdot 8 \\
(443 \cdot 0 \text { to } \\
873 \cdot 1)\end{array}$ & $-5 \cdot 7(-13 \cdot 1$ to $-0 \cdot 5) *$ & $\begin{array}{l}9 \cdot 9(7 \cdot 0 \\
\text { to } 13 \cdot 6)\end{array}$ & $\begin{array}{l}8 \cdot 5(6 \cdot 0 \\
\text { to } 11 \cdot 7)\end{array}$ & $\begin{array}{c}-14 \cdot 3(-21 \cdot 0 \text { to } \\
-9 \cdot 5)^{*}\end{array}$ \\
\hline $\begin{array}{l}\text { Haemoglobinopathies and } \\
\text { haemolytic anaemias }\end{array}$ & $\begin{array}{c}20495 \cdot 6 \\
(12009 \cdot 2 \\
\text { to } 32 \\
293 \cdot 0)\end{array}$ & $\begin{array}{c}23368 \cdot 9 \\
(12797 \cdot 5 \\
\text { to } 39 \\
245 \cdot 7)\end{array}$ & $11.9(0.7 \text { to } 33.0)^{*}$ & $\begin{array}{c}302 \cdot 4 \\
(178 \cdot 3 \text { to } \\
473 \cdot 5)\end{array}$ & $\begin{array}{c}322 \cdot 0 \\
(177 \cdot 3 \text { to } \\
538 \cdot 3)\end{array}$ & $4 \cdot 4(-5 \cdot 9$ to $24 \cdot 2)$ \\
\hline
\end{tabular}




\begin{tabular}{|c|c|c|c|c|c|c|}
\hline & \multicolumn{3}{|c|}{ All ages DALYs (thousands) ${ }^{*}$} & \multicolumn{3}{|c|}{ Age-standardised DALYs (per 100000 ) } \\
\hline & 2005 & 2013 & Percentage change & 2005 & 2013 & Percentage change \\
\hline Thalassaemias & $\begin{array}{l}2096 \cdot 3 \\
(1466 \cdot 2 \text { to } \\
2677 \cdot 9)\end{array}$ & $\begin{array}{l}1814 \cdot 1 \\
(1257 \cdot 4 \text { to } \\
2332 \cdot 0)\end{array}$ & $-15 \cdot 4(-23 \cdot 1$ to $4 \cdot 8)$ & $\begin{array}{l}30 \cdot 1 \\
(21 \cdot 0 \text { to } \\
38 \cdot 5)\end{array}$ & $\begin{array}{l}24 \cdot 7 \\
(17 \cdot 1 \text { to } \\
31 \cdot 7)\end{array}$ & $\begin{array}{c}-19 \cdot 9(-27 \cdot 1 \text { to } \\
-0 \cdot 6)^{*}\end{array}$ \\
\hline Thalassaemia trait & $\begin{array}{l}3401 \cdot 3 \\
(2267 \cdot 4 \text { to } \\
4899 \cdot 0)\end{array}$ & $\begin{array}{c}3769 \cdot 6 \\
(2508 \cdot 9 \text { to } \\
5442 \cdot 2)\end{array}$ & $10 \cdot 8(8 \cdot 1 \text { to } 13 \cdot 8)^{*}$ & $\begin{array}{l}51 \cdot 0 \\
(34 \cdot 1 \text { to } \\
73 \cdot 6)\end{array}$ & $\begin{array}{l}52 \cdot 4 \\
(34 \cdot 9 \text { to } \\
75 \cdot 7)\end{array}$ & $2.7(0.1 \text { to } 5.5)^{*}$ \\
\hline Sickle cell disorders & $\begin{array}{l}11142 \cdot 6 \\
(3743 \cdot 2 \text { to } \\
22090 \cdot 0)\end{array}$ & $\begin{array}{l}13650 \cdot 5 \\
(4382 \cdot 8 \text { to } \\
29097 \cdot 8)\end{array}$ & $20 \cdot 8(0 \cdot 1 \text { to } 52 \cdot 5)^{*}$ & $\begin{array}{l}161 \cdot 9 \\
(54 \cdot 6 \text { to } \\
320 \cdot 2)\end{array}$ & $\begin{array}{l}186 \cdot 5 \\
(59 \cdot 8 \text { to } \\
397 \cdot 2)\end{array}$ & $13 \cdot 4(-6 \cdot 1$ to $43 \cdot 9)$ \\
\hline Sickle cell trait & $\begin{array}{l}1251 \cdot 0 \\
(829 \cdot 2 \text { to } \\
1811 \cdot 0)\end{array}$ & $\begin{array}{l}1396 \cdot 6 \\
(929 \cdot 4 \text { to } \\
2004 \cdot 9)\end{array}$ & $11.5(6.2 \text { to } 19.0)^{*}$ & $\begin{array}{l}18 \cdot 4 \\
(12 \cdot 2 \text { to } \\
26 \cdot 7)\end{array}$ & $\begin{array}{l}19 \cdot 3 \\
(12 \cdot 9 \text { to } \\
27 \cdot 8)\end{array}$ & $4 \cdot 8(-0 \cdot 1$ to $11 \cdot 8)$ \\
\hline Glucose-6-phosphate dehydrogen & $\begin{array}{l}\text { ase Reffisclency } \\
(176 \cdot 5 \text { to } \\
341 \cdot 5)\end{array}$ & $\begin{array}{c}269 \cdot 1 \\
(174 \cdot 8 \text { to } \\
371 \cdot 8)\end{array}$ & $-0.7(-11.3$ to 22.7$)$ & $\begin{array}{l}4 \cdot 0(2 \cdot 6 \\
\text { to } 5 \cdot 1)\end{array}$ & $\begin{array}{l}3.7(2 \cdot 4 \\
\text { to } 5 \cdot 1)\end{array}$ & $-8 \cdot 2(-17 \cdot 9$ to $12 \cdot 8$ \\
\hline $\begin{array}{c}\text { Glucose-6-phosphate } \\
\text { dehydrogenase deficiency trait }\end{array}$ & $\begin{array}{l}44 \cdot 6(27 \cdot 7 \\
\text { to } 66 \cdot 0)\end{array}$ & $\begin{array}{l}48 \cdot 8(30 \cdot 1 \\
\text { to } 73 \cdot 3)\end{array}$ & $9 \cdot 2(-12 \cdot 1$ to $34 \cdot 0)$ & $\begin{array}{l}0.7(0.4 \\
\text { to } 1 \cdot 0)\end{array}$ & $\begin{array}{l}0.7(0 \cdot 4 \\
\text { to } 1 \cdot 0)\end{array}$ & $2 \cdot 4(-17 \cdot 6$ to $25 \cdot 1)$ \\
\hline $\begin{array}{l}\text { Other haemoglobinopathies and } \\
\text { haemolytic anaemias }\end{array}$ & $\begin{array}{l}2294 \cdot 8 \\
(1707 \cdot 8 \text { to } \\
2962 \cdot 2)\end{array}$ & $\begin{array}{l}2420 \cdot 1 \\
(1814 \cdot 8 \text { to } \\
3146 \cdot 8)\end{array}$ & $5 \cdot 1(-1 \cdot 0$ to $13 \cdot 6)$ & $\begin{array}{l}36 \cdot 4 \\
(27 \cdot 2 \text { to } \\
46 \cdot 8)\end{array}$ & $\begin{array}{l}34 \cdot 7 \\
(26 \cdot 2 \text { to } \\
45 \cdot 1)\end{array}$ & $-4 \cdot 9(-10 \cdot 2$ to $2 \cdot 2)$ \\
\hline $\begin{array}{l}\text { Endocrine, metabolic, blood, and } \\
\text { immune disorders }\end{array}$ & $\begin{array}{l}8175 \cdot 5 \\
(7030 \cdot 5 \text { to } \\
9563 \cdot 7)\end{array}$ & $\begin{array}{c}8987 \cdot 0 \\
(7724 \cdot 2 \text { to } \\
10348 \cdot 0)\end{array}$ & $10 \cdot 1(1.9 \text { to } 17.8)^{*}$ & $\begin{array}{l}130 \cdot 6 \\
(111 \cdot 8 \text { to } \\
152 \cdot 6)\end{array}$ & $\begin{array}{l}128 \cdot 9 \\
(110 \cdot 9 \text { to } \\
148 \cdot 3)\end{array}$ & $-1 \cdot 1(-8 \cdot 2$ to $5 \cdot 4)$ \\
\hline Musculoskeletal disorders & $\begin{array}{c}126874 \cdot 2 \\
(91296 \cdot 7 \\
\text { to } 167 \\
000 \cdot 8)\end{array}$ & $\begin{array}{c}149435 \cdot 7 \\
(106 \\
888 \cdot 5 \text { to } \\
197 \\
565 \cdot 1)\end{array}$ & $17.7(16.2 \text { to } 19.8)^{*}$ & $\begin{array}{c}2162 \cdot 3 \\
(1559 \cdot 6 \\
\text { to } \\
2834 \cdot 5)\end{array}$ & $\begin{array}{c}2178 \cdot 0 \\
(1561 \cdot 5 \\
\text { to } \\
2875 \cdot 0)\end{array}$ & $0 \cdot 6(-0.5$ to $2 \cdot 4)$ \\
\hline Rheumatoid arthritis & $\begin{array}{l}4299 \cdot 0 \\
(3304 \cdot 9 \text { to } \\
5461 \cdot 2)\end{array}$ & $\begin{array}{l}4741 \cdot 2 \\
(3597 \cdot 6 \text { to } \\
5988 \cdot 1)\end{array}$ & $10 \cdot 2(7.5 \text { to } 13.5)^{*}$ & $\begin{array}{l}78 \cdot 0 \\
(60 \cdot 2 \text { to } \\
98 \cdot 8)\end{array}$ & $\begin{array}{l}72.5 \\
(55 \cdot 1 \text { to } \\
91 \cdot 3)\end{array}$ & $-7.2(-9.5 \text { to }-4.4)^{*}$ \\
\hline Osteoarthritis & $\begin{array}{c}10401 \cdot 5 \\
(7337 \cdot 3 \text { to } \\
14133 \cdot 8)\end{array}$ & $\begin{array}{l}12811 \cdot 1 \\
(9030 \cdot 0 \text { to } \\
17281 \cdot 2)\end{array}$ & $23.2(21.6 \text { to } 24.7)^{*}$ & $\begin{array}{l}201 \cdot 3 \\
(142 \cdot 1 \text { to } \\
272 \cdot 9)\end{array}$ & $\begin{array}{c}201 \cdot 7 \\
(142 \cdot 3 \text { to } \\
271 \cdot 8)\end{array}$ & $0 \cdot 2(-1 \cdot 1$ to $1 \cdot 5)$ \\
\hline Low back and neck pain & $\begin{array}{c}91729 \cdot 2 \\
(64002 \cdot 3 \\
\text { to } 123 \\
315 \cdot 7)\end{array}$ & $\begin{array}{c}106665 \cdot 5 \\
(74116 \cdot 9 \\
\text { to } 142 \\
959 \cdot 7)\end{array}$ & $16 \cdot 2(14.0 \text { to } 19 \cdot 2)^{*}$ & $\begin{array}{c}1525 \cdot 7 \\
(1066 \cdot 3 \\
\text { to } \\
2048 \cdot 4)\end{array}$ & $\begin{array}{c}1532 \cdot 8 \\
(1065 \cdot 6 \\
\text { to } \\
2052 \cdot 0)\end{array}$ & $0.4(-1 \cdot 4$ to $2 \cdot 9)$ \\
\hline Low back pain & $\begin{array}{c}61611 \cdot 0 \\
(42074 \cdot 7 \\
\text { to } 84 \\
850 \cdot 7)\end{array}$ & $\begin{array}{c}72317 \cdot 6 \\
(49051 \cdot 0 \\
\text { to } 99 \\
738 \cdot 5)\end{array}$ & $17 \cdot 2(14.9 \text { to } 21 \cdot 1)^{*}$ & $\begin{array}{c}1032 \cdot 4 \\
(705 \cdot 9 \text { to } \\
1418 \cdot 2)\end{array}$ & $\begin{array}{c}1045 \cdot 3 \\
(710 \cdot 2 \text { to } \\
1440 \cdot 6)\end{array}$ & $1 \cdot 0(-0 \cdot 8$ to $4 \cdot 4)$ \\
\hline Neck pain & $\begin{array}{c}30118 \cdot 2 \\
(20855 \cdot 1 \\
\text { to } 41 \\
090 \cdot 2)\end{array}$ & $\begin{array}{c}34347 \cdot 9 \\
(23792 \cdot 0 \\
\text { to } 47 \\
418 \cdot 5)\end{array}$ & $14.1(10 \cdot 1 \text { to } 18.3)^{*}$ & $\begin{array}{c}493 \cdot 3 \\
(342 \cdot 4 \text { to } \\
672 \cdot 2)\end{array}$ & $\begin{array}{c}487.5 \\
(337.8 \text { to } \\
672.2)\end{array}$ & $-1 \cdot 1(-4 \cdot 5$ to $2 \cdot 5)$ \\
\hline Gout & $\begin{array}{c}154 \cdot 3 \\
(106 \cdot 8 \text { to } \\
205 \cdot 2)\end{array}$ & $\begin{array}{c}185 \cdot 5 \\
(129 \cdot 0 \text { to } \\
249 \cdot 2)\end{array}$ & $20 \cdot 1(16 \cdot 5 \text { to } 24 \cdot 5)^{*}$ & $\begin{array}{l}2 \cdot 9(2 \cdot 0 \\
\text { to } 3 \cdot 8)\end{array}$ & $\begin{array}{l}2 \cdot 8(2 \cdot 0 \\
\text { to } 3 \cdot 8)\end{array}$ & $-0 \cdot 8(-3 \cdot 7$ to $2 \cdot 8)$ \\
\hline Other musculoskeletal disorders & $\begin{array}{c}20290 \cdot 1 \\
(14431 \cdot 4 \\
\text { to } 27 \\
422 \cdot 7)\end{array}$ & $\begin{array}{c}25032 \cdot 4 \\
(17671 \cdot 6 \\
\text { to } 34 \\
085 \cdot 8)\end{array}$ & $23.3(21.2 \text { to } 25.5)^{*}$ & $\begin{array}{c}354.4 \\
(251 \cdot 9 \text { to } \\
481 \cdot 1)\end{array}$ & $\begin{array}{c}368 \cdot 3 \\
(260 \cdot 1 \text { to } \\
502 \cdot 1)\end{array}$ & $3.9(2.4 \text { to } 5.6)^{*}$ \\
\hline Other non-communicable diseases & $\begin{array}{c}157897 \cdot 8 \\
(120 \\
772 \cdot 1 \text { to }\end{array}$ & $\begin{array}{c}170947.9 \\
(130 \\
922 \cdot 9 \text { to }\end{array}$ & $8.7(3.1 \text { to } 11 \cdot 6)^{*}$ & $\begin{array}{c}2549 \cdot 4 \\
(1934 \cdot 9 \\
\text { to } \\
3320 \cdot 6)\end{array}$ & $\begin{array}{c}2465 \cdot 7 \\
(1884 \cdot 2 \\
\text { to } \\
3234 \cdot 3)\end{array}$ & $-3 \cdot 0(-7 \cdot 3 \text { to }-0 \cdot 6)^{*}$ \\
\hline
\end{tabular}




\begin{tabular}{|c|c|c|c|c|c|c|}
\hline & \multicolumn{3}{|c|}{ All ages DALYs (thousands) ${ }^{*}$} & \multicolumn{3}{|c|}{ Age-standardised DALYs (per 100000$)^{*}$} \\
\hline & 2005 & 2013 & Percentage change & 2005 & 2013 & Percentage change \\
\hline & $\begin{array}{c}204 \\
769 \cdot 6)\end{array}$ & $\begin{array}{c}223 \\
484 \cdot 3)\end{array}$ & & & & \\
\hline Congenital anomalies & $\begin{array}{l}56944 \cdot 6 \\
(49141 \cdot 0 \\
\text { to } 69 \\
460 \cdot 2)\end{array}$ & $\begin{array}{l}57173 \cdot 2 \\
(50550 \cdot 4 \\
\text { to } 66 \\
265 \cdot 6)\end{array}$ & $1 \cdot 6(-10 \cdot 4$ to $9 \cdot 0)$ & $\begin{array}{c}827 \cdot 1 \\
(714 \cdot 5 \text { to } \\
1007 \cdot 5)\end{array}$ & $\begin{array}{c}779 \cdot 9 \\
(689 \cdot 9 \text { to } \\
903 \cdot 0)\end{array}$ & $-4 \cdot 6(-15 \cdot 8$ to $2 \cdot 4)$ \\
\hline Neural tube defects & $\begin{array}{c}7161 \cdot 8 \\
(4444 \cdot 2 \text { to } \\
12759 \cdot 5)\end{array}$ & $\begin{array}{c}6236 \cdot 6 \\
(3854 \cdot 9 \text { to } \\
10918 \cdot 2)\end{array}$ & $-12 \cdot 7(-28 \cdot 6$ to $4 \cdot 8)$ & $\begin{array}{l}102 \cdot 6 \\
(63 \cdot 7 \text { to } \\
183 \cdot 0)\end{array}$ & $\begin{array}{c}84 \cdot 4 \\
(52 \cdot 2 \text { to } \\
147 \cdot 9)\end{array}$ & $\begin{array}{c}-17.4(-32.5 \text { to } \\
-0.9)^{*}\end{array}$ \\
\hline Congenital heart anomalies & $\begin{array}{l}26144 \cdot 1 \\
(22321 \cdot 2 \\
\text { to } 32 \\
868 \cdot 8)\end{array}$ & $\begin{array}{l}26219 \cdot 2 \\
(23222 \cdot 4 \\
\text { to } 30 \\
340 \cdot 6)\end{array}$ & $1 \cdot 7(-12 \cdot 6$ to $11 \cdot 8)$ & $\begin{array}{c}377 \cdot 4 \\
(322 \cdot 2 \text { to } \\
473 \cdot 6)\end{array}$ & $\begin{array}{c}356 \cdot 6 \\
(316 \cdot 0 \text { to } \\
412 \cdot 7)\end{array}$ & $-4 \cdot 1(-17 \cdot 6$ to $5 \cdot 4)$ \\
\hline Orofacial clefts & $\begin{array}{c}416 \cdot 1 \\
(257 \cdot 1 \text { to } \\
602 \cdot 1)\end{array}$ & $\begin{array}{c}352 \cdot 3 \\
(229 \cdot 2 \text { to } \\
515 \cdot 7)\end{array}$ & $-15 \cdot 6(-31 \cdot 8$ to $8 \cdot 8)$ & $\begin{array}{c}6 \cdot 0(3.7 \\
\text { to } 8 \cdot 6)\end{array}$ & $\begin{array}{c}4 \cdot 8(3 \cdot 1 \\
\text { to } 7 \cdot 0)\end{array}$ & $-20 \cdot 3(-35 \cdot 5$ to $2 \cdot 6)$ \\
\hline Down's syndrome & $\begin{array}{c}3578 \cdot 6 \\
(2139 \cdot 0 \text { to } \\
5189 \cdot 6)\end{array}$ & $\begin{array}{c}3851 \cdot 4 \\
(2556 \cdot 6 \text { to } \\
5223 \cdot 2)\end{array}$ & $8 \cdot 7(-8 \cdot 6$ to $27 \cdot 8)$ & $\begin{array}{l}53 \cdot 5 \\
(32 \cdot 8 \text { to } \\
76 \cdot 8)\end{array}$ & $\begin{array}{c}53 \cdot 2 \\
(35 \cdot 5 \text { to } \\
71 \cdot 8)\end{array}$ & $0.5(-14.9$ to $17 \cdot 5)$ \\
\hline Turner's syndrome & $\begin{array}{c}3 \cdot 8(1.9 \text { to } \\
6 \cdot 1)\end{array}$ & $\begin{array}{c}4.3(2.2 \text { to } \\
6 \cdot 9)\end{array}$ & $13 \cdot 3(5 \cdot 1 \text { to } 23 \cdot 2)^{*}$ & $\begin{array}{c}0 \cdot 1(0 \cdot 0 \\
\text { to } 0 \cdot 1)\end{array}$ & $\begin{array}{c}0 \cdot 1(0 \cdot 0 \\
\text { to } 0 \cdot 1)\end{array}$ & $4 \cdot 4(-3 \cdot 3$ to $13 \cdot 4)$ \\
\hline Klinefelter's syndrome & $\begin{array}{c}1 \cdot 2(0 \cdot 6 \text { to } \\
2 \cdot 2)\end{array}$ & $\begin{array}{c}1.3(0.6 \text { to } \\
2 \cdot 4)\end{array}$ & $13.0(5 \cdot 2 \text { to } 21 \cdot 5)^{*}$ & $\begin{array}{c}0 \cdot 0(0 \cdot 0 \\
\text { to } 0 \cdot 0)\end{array}$ & $\begin{array}{c}0 \cdot 0(0 \cdot 0 \\
\text { to } 0 \cdot 0)\end{array}$ & $2 \cdot 1(-4 \cdot 9$ to $9 \cdot 6)$ \\
\hline Chromosomal unbalanced rear & $\begin{array}{c}\text { gendent } 19.6 \\
3774 \cdot 0)\end{array}$ & $\begin{array}{c}2985 \cdot 4 \\
(2313 \cdot 0 \text { to } \\
3917 \cdot 7)\end{array}$ & $16 \cdot 2(-2 \cdot 6$ to $25 \cdot 0)$ & $\begin{array}{c}40 \cdot 2 \\
(29 \cdot 9 \text { to } \\
56 \cdot 8)\end{array}$ & $\begin{array}{c}41 \cdot 7 \\
(32 \cdot 4 \text { to } \\
54 \cdot 6)\end{array}$ & $5 \cdot 8(-9 \cdot 8$ to $13 \cdot 3)$ \\
\hline Other congenital anomalies & $\begin{array}{c}17009 \cdot 4 \\
(13826 \cdot 2 \\
\text { to } 25 \\
442 \cdot 3)\end{array}$ & $\begin{array}{c}17522 \cdot 7 \\
(14425 \cdot 9 \\
\text { to } 24 \\
552 \cdot 1)\end{array}$ & $4 \cdot 1(-9 \cdot 1$ to $14 \cdot 4)$ & $\begin{array}{c}247 \cdot 4 \\
(201 \cdot 2 \text { to } \\
368 \cdot 7)\end{array}$ & $\begin{array}{c}239 \cdot 1 \\
(196 \cdot 7 \text { to } \\
334 \cdot 7)\end{array}$ & $-2 \cdot 4(-14 \cdot 5$ to $7 \cdot 1)$ \\
\hline Skin and subcutaneous diseases & $\begin{array}{c}37827 \cdot 9 \\
(25158 \cdot 8 \\
\text { to } 56 \\
628 \cdot 8)\end{array}$ & $\begin{array}{c}41597 \cdot 6 \\
(27763 \cdot 0 \\
\text { to } 62 \\
743 \cdot 1)\end{array}$ & $10 \cdot 0(7 \cdot 8 \text { to } 12 \cdot 0)^{*}$ & $\begin{array}{c}582 \cdot 5 \\
(390 \cdot 2 \text { to } \\
865 \cdot 7)\end{array}$ & $\begin{array}{c}582 \cdot 9 \\
(390 \cdot 1 \text { to } \\
872 \cdot 3)\end{array}$ & $0 \cdot 1(-1 \cdot 8$ to $1 \cdot 8)$ \\
\hline Dermatitis & $\begin{array}{c}8431 \cdot 6 \\
(5490 \cdot 7 \text { to } \\
12137 \cdot 7)\end{array}$ & $\begin{array}{c}9278 \cdot 4 \\
(6029 \cdot 0 \text { to } \\
13326 \cdot 7)\end{array}$ & $10 \cdot 0(9 \cdot 2 \text { to } 11 \cdot 0)^{*}$ & $\begin{array}{c}128 \cdot 6 \\
(83 \cdot 5 \text { to } \\
184 \cdot 8)\end{array}$ & $\begin{array}{c}128 \cdot 7 \\
(83 \cdot 6 \text { to } \\
184 \cdot 9)\end{array}$ & $0 \cdot 1(-0.4$ to $0 \cdot 6)$ \\
\hline Psoriasis & $\begin{array}{c}4187 \cdot 6 \\
(2896 \cdot 0 \text { to } \\
5899 \cdot 7)\end{array}$ & $\begin{array}{c}4726 \cdot 7 \\
(3254 \cdot 7 \text { to } \\
6621 \cdot 9)\end{array}$ & $12.9(11.4 \text { to } 14.4)^{*}$ & $\begin{array}{c}67 \cdot 2 \\
(46 \cdot 4 \text { to } \\
94 \cdot 6)\end{array}$ & $\begin{array}{c}66 \cdot 8 \\
(46 \cdot 0 \text { to } \\
93 \cdot 6)\end{array}$ & $-0.5(-1.6$ to $0 \cdot 6)$ \\
\hline Cellulitis & $\begin{array}{c}1083 \cdot 0 \\
(809 \cdot 2 \text { to } \\
1402 \cdot 4)\end{array}$ & $\begin{array}{c}1064 \cdot 7 \\
(814 \cdot 0 \text { to } \\
1397 \cdot 5)\end{array}$ & $-2 \cdot 3(-11 \cdot 5$ to $11 \cdot 1)$ & $\begin{array}{c}17 \cdot 7 \\
(13 \cdot 1 \text { to } \\
23 \cdot 0)\end{array}$ & $\begin{array}{c}15 \cdot 5 \\
(11 \cdot 8 \text { to } \\
20 \cdot 2)\end{array}$ & $\begin{array}{c}-13 \cdot 2(-21 \cdot 1 \text { to } \\
-1 \cdot 7)^{*}\end{array}$ \\
\hline Pyoderma & $\begin{array}{c}943 \cdot 5 \\
(698 \cdot 5 \text { to } \\
1195 \cdot 4)\end{array}$ & $\begin{array}{c}1141 \cdot 6 \\
(888 \cdot 5 \text { to } \\
1330 \cdot 2)\end{array}$ & $21.4(4.7 \text { to } 39.8)^{*}$ & $\begin{array}{c}15 \cdot 7 \\
(11.7 \text { to } \\
19 \cdot 6)\end{array}$ & $\begin{array}{c}16 \cdot 6 \\
(13 \cdot 0 \text { to } \\
19 \cdot 3)\end{array}$ & $6 \cdot 4(-7 \cdot 2$ to $21 \cdot 3)$ \\
\hline Scabies & $\begin{array}{l}1624 \cdot 7 \\
(927 \cdot 0 \text { to } \\
2620 \cdot 2)\end{array}$ & $\begin{array}{l}1705 \cdot 4 \\
(967 \cdot 2 \text { to } \\
2711 \cdot 6)\end{array}$ & $4 \cdot 8(-3 \cdot 4$ to $15 \cdot 1)$ & $\begin{array}{c}24 \cdot 1 \\
(13 \cdot 8 \text { to } \\
38 \cdot 9)\end{array}$ & $\begin{array}{c}23 \cdot 5 \\
(13 \cdot 3 \text { to } \\
37 \cdot 3)\end{array}$ & $-2 \cdot 8(-10 \cdot 2$ to $6 \cdot 7)$ \\
\hline Fungal skin diseases & $\begin{array}{c}3447 \cdot 0 \\
(1403 \cdot 8 \text { to } \\
7290 \cdot 4)\end{array}$ & $\begin{array}{c}3847 \cdot 2 \\
(1574 \cdot 5 \text { to } \\
8139 \cdot 8)\end{array}$ & $11.6(10 \cdot 5 \text { to } 12 \cdot 8)^{*}$ & $\begin{array}{c}53 \cdot 4 \\
(21.8 \text { to } \\
113 \cdot 0)\end{array}$ & $\begin{array}{c}54 \cdot 0 \\
(22 \cdot 1 \text { to } \\
114 \cdot 2)\end{array}$ & $1.0(0.5 \text { to } 1.4)^{*}$ \\
\hline Viral skin diseases & $\begin{array}{c}3752 \cdot 1 \\
(2284 \cdot 2 \text { to } \\
5820 \cdot 4)\end{array}$ & $\begin{array}{c}3955 \cdot 0 \\
(2398.4 \text { to } \\
6150 \cdot 9)\end{array}$ & $5.4(4.1 \text { to } 6 \cdot 7)^{*}$ & $\begin{array}{c}55 \cdot 1 \\
(33 \cdot 4 \text { to } \\
85 \cdot 5)\end{array}$ & $\begin{array}{c}54 \cdot 7 \\
(33 \cdot 3 \text { to } \\
85 \cdot 0)\end{array}$ & $-0.6(-1.5$ to $0 \cdot 3)$ \\
\hline
\end{tabular}




\begin{tabular}{|c|c|c|c|c|c|c|}
\hline & \multicolumn{3}{|c|}{ All ages DALYs (thousands) ${ }^{*}$} & \multicolumn{3}{|c|}{ Age-standardised DALYs (per 100000$)^{*}$} \\
\hline & 2005 & 2013 & Percentage change & 2005 & 2013 & Percentage change \\
\hline Acne vulgaris & $\begin{array}{c}6982 \cdot 2 \\
(3360 \cdot 8 \text { to } \\
12916 \cdot 9)\end{array}$ & $\begin{array}{c}7180 \cdot 8 \\
(3451 \cdot 6 \text { to } \\
13214 \cdot 1)\end{array}$ & $2.5(-3 \cdot 7$ to $11 \cdot 1)$ & $\begin{array}{c}96 \cdot 9 \\
(46 \cdot 7 \text { to } \\
179 \cdot 1)\end{array}$ & $\begin{array}{c}96 \cdot 7 \\
(46.4 \text { to } \\
177 \cdot 8)\end{array}$ & $-0.5(-6 \cdot 5$ to $7 \cdot 7)$ \\
\hline Alopecia areata & $\begin{array}{c}257.7 \\
(163.9 \text { to } \\
382 \cdot 5)\end{array}$ & $\begin{array}{c}292 \cdot 4 \\
(186 \cdot 8 \text { to } \\
435 \cdot 2)\end{array}$ & $13.5(10.3 \text { to } 16 \cdot 8)^{*}$ & $\begin{array}{l}4 \cdot 2(2 \cdot 7 \\
\text { to } 6 \cdot 3)\end{array}$ & $\begin{array}{l}4 \cdot 2(2 \cdot 7 \\
\text { to } 6 \cdot 3)\end{array}$ & $-0 \cdot 1(-2 \cdot 9$ to $2 \cdot 7)$ \\
\hline Pruritus & $\begin{array}{c}9 \cdot 2(4 \cdot 4 \text { to } \\
17 \cdot 1)\end{array}$ & $\begin{array}{l}10 \cdot 8(5 \cdot 1 \\
\text { to } 20 \cdot 0)\end{array}$ & $17.4(9 \cdot 2 \text { to } 25 \cdot 7)^{*}$ & $\begin{array}{l}0 \cdot 2(0 \cdot 1 \\
\text { to } 0 \cdot 3)\end{array}$ & $\begin{array}{l}0 \cdot 2(0 \cdot 1 \\
\text { to } 0 \cdot 3)\end{array}$ & $0 \cdot 9(-6 \cdot 2$ to $8 \cdot 6)$ \\
\hline Urticaria & $\begin{array}{c}3993 \cdot 4 \\
(2616 \cdot 6 \text { to } \\
5702 \cdot 1)\end{array}$ & $\begin{array}{c}4720 \cdot 7 \\
(3036 \cdot 5 \text { to } \\
6737 \cdot 2)\end{array}$ & $19.0(2.7 \text { to } 32.6)^{*}$ & $\begin{array}{l}64 \cdot 6 \\
(42 \cdot 5 \text { to } \\
92 \cdot 2)\end{array}$ & $\begin{array}{l}67 \cdot 0 \\
(43 \cdot 2 \text { to } \\
95 \cdot 5)\end{array}$ & $4 \cdot 3(-9 \cdot 4$ to $16 \cdot 1)$ \\
\hline Decubitus ulcer & $\begin{array}{c}546 \cdot 8 \\
(454 \cdot 9 \text { to } \\
647 \cdot 3)\end{array}$ & $\begin{array}{c}660 \cdot 6 \\
(553 \cdot 9 \text { to } \\
782 \cdot 1)\end{array}$ & $20.9(14.5 \text { to } 27 \cdot 7)^{*}$ & $\begin{array}{l}10 \cdot 9(9 \cdot 1 \\
\text { to } 12 \cdot 8)\end{array}$ & $\begin{array}{l}10 \cdot 8(9 \cdot 1 \\
\text { to } 12 \cdot 7)\end{array}$ & $-0 \cdot 8(-5.8$ to $4 \cdot 6)$ \\
\hline $\begin{array}{l}\text { Other skin and subcutaneous } \\
\text { diseases }\end{array}$ & $\begin{array}{c}2569 \cdot 0 \\
(1164 \cdot 2 \text { to } \\
5187 \cdot 3)\end{array}$ & $\begin{array}{c}3013 \cdot 3 \\
(1374 \cdot 5 \text { to } \\
6234 \cdot 7)\end{array}$ & $17.2(13.2 \text { to } 20.7)^{*}$ & $\begin{array}{c}44 \cdot 0 \\
(19 \cdot 4 \text { to } \\
93 \cdot 1)\end{array}$ & $\begin{array}{c}44 \cdot 2 \\
(19 \cdot 8 \text { to } \\
93 \cdot 3)\end{array}$ & $0.4(-0.7$ to 1.6$)$ \\
\hline Sense organ diseases & $\begin{array}{c}47426 \cdot 9 \\
(31917 \cdot 8 \\
\text { to } 66 \\
335 \cdot 1)\end{array}$ & $\begin{array}{c}54428 \cdot 1 \\
(36458 \cdot 4 \\
\text { to } 76 \\
075 \cdot 4)\end{array}$ & $14.7(13.2 \text { to } 16 \cdot 2)^{*}$ & $\begin{array}{c}868 \cdot 4 \\
(588 \cdot 1 \text { to } \\
1206 \cdot 6)\end{array}$ & $\begin{array}{c}839.3 \\
(564.9 \text { to } \\
1165.9)\end{array}$ & $-3.4(-4.6 \text { to }-2 \cdot 2)^{*}$ \\
\hline Glaucoma & $\begin{array}{c}701.8 \\
(496.4 \text { to } \\
963.7)\end{array}$ & $\begin{array}{c}807.5 \\
(571 \cdot 6 \text { to } \\
1102 \cdot 8)\end{array}$ & $15 \cdot 0(10 \cdot 0 \text { to } 20 \cdot 2)^{*}$ & $\begin{array}{l}13 \cdot 3(9 \cdot 5 \\
\text { to } 18 \cdot 2)\end{array}$ & $\begin{array}{l}12 \cdot 7(9 \cdot 0 \\
\text { to } 17 \cdot 3)\end{array}$ & $-5 \cdot 1(-9 \cdot 4$ to $-1 \cdot 0) *$ \\
\hline Cataract & $\begin{array}{c}2592.9 \\
(1853 \cdot 6 \text { to } \\
3530 \cdot 8)\end{array}$ & $\begin{array}{c}2916 \cdot 7 \\
(2055 \cdot 1 \text { to } \\
3962 \cdot 2)\end{array}$ & $12.5(8 \cdot 2 \text { to } 16 \cdot 9)^{*}$ & $\begin{array}{c}54 \cdot 1 \\
(38 \cdot 6 \text { to } \\
73 \cdot 4)\end{array}$ & $\begin{array}{c}49 \cdot 0 \\
(34 \cdot 7 \text { to } \\
66 \cdot 4)\end{array}$ & $-9.4(-12.8 \text { to }-5.9)^{*}$ \\
\hline Macular degeneration & $\begin{array}{c}516 \cdot 8 \\
(368 \cdot 3 \text { to } \\
697 \cdot 4)\end{array}$ & $\begin{array}{c}725 \cdot 6 \\
(509 \cdot 4 \text { to } \\
985 \cdot 1)\end{array}$ & $40.4(33.8 \text { to } 47.0)^{*}$ & $\begin{array}{l}10 \cdot 8(7 \cdot 7 \\
\text { to } 14 \cdot 5)\end{array}$ & $\begin{array}{l}11.9(8.4 \\
\text { to } 16 \cdot 2)\end{array}$ & $10 \cdot 6(5 \cdot 1 \text { to } 16 \cdot 0)^{*}$ \\
\hline Uncorrected refractive error & $\begin{array}{c}10004 \cdot 7 \\
(6360 \cdot 2 \text { to } \\
15412 \cdot 8)\end{array}$ & $\begin{array}{c}11257 \cdot 2 \\
(7149 \cdot 8 \text { to } \\
17452 \cdot 3)\end{array}$ & $12.5(10.7 \text { to } 14.3)^{*}$ & $\begin{array}{c}176 \cdot 9 \\
(112 \cdot 7 \text { to } \\
272 \cdot 8)\end{array}$ & $\begin{array}{c}169.2 \\
(107 \cdot 6 \text { to } \\
261.9)\end{array}$ & $-4.4(-5.7 \text { to }-3.0)^{*}$ \\
\hline Age-related and other hearing & $\begin{array}{c}28010 \cdot 5 \\
(18942 \cdot 1 \\
\text { to } 39 \\
007 \cdot 8)\end{array}$ & $\begin{array}{c}32579 \cdot 7 \\
(22083 \cdot 7 \\
\text { to } 45 \\
846 \cdot 1)\end{array}$ & $16.3(13.9 \text { to } 18.8)^{*}$ & $\begin{array}{c}521.4 \\
(355 \cdot 2 \text { to } \\
721.5)\end{array}$ & $\begin{array}{c}507 \cdot 3 \\
(346 \cdot 5 \text { to } \\
710 \cdot 4)\end{array}$ & $-2.7(-4.5$ to -0.9$) *$ \\
\hline Other vision loss & $\begin{array}{l}1690 \cdot 5 \\
(1191 \cdot 0 \text { to } \\
2309 \cdot 3)\end{array}$ & $\begin{array}{c}1793 \cdot 5 \\
(1260 \cdot 4 \text { to } \\
2452 \cdot 0)\end{array}$ & $6.0(2.8 \text { to } 9.7)^{*}$ & $\begin{array}{c}29 \cdot 8 \\
(21 \cdot 1 \text { to } \\
40 \cdot 7)\end{array}$ & $\begin{array}{c}27 \cdot 1 \\
(19 \cdot 1 \text { to } \\
36 \cdot 9)\end{array}$ & $-9.4(-11.8 \text { to }-6.5)^{*}$ \\
\hline Other sense organ diseases & $\begin{array}{c}3909.6 \\
(2421.5 \text { to } \\
5767.2)\end{array}$ & $\begin{array}{c}4348 \cdot 0 \\
(2704 \cdot 3 \text { to } \\
6435 \cdot 1)\end{array}$ & $11.2(9.5 \text { to } 12.9)^{*}$ & $\begin{array}{l}62 \cdot 1 \\
(38.5 \text { to } \\
91 \cdot 5)\end{array}$ & $\begin{array}{l}62.2 \\
(38.7 \text { to } \\
91.9)\end{array}$ & $0 \cdot 1(-1 \cdot 3$ to $1 \cdot 6)$ \\
\hline Oral disorders & $\begin{array}{l}14385 \cdot 1 \\
(8778 \cdot 2 \text { to } \\
22332 \cdot 8)\end{array}$ & $\begin{array}{c}16449 \cdot 5 \\
(10022 \cdot 3 \\
\text { to } 25 \\
506 \cdot 3)\end{array}$ & $14.3(13.2 \text { to } 15.5)^{*}$ & $\begin{array}{c}252 \cdot 5 \\
(156 \cdot 1 \text { to } \\
388 \cdot 1)\end{array}$ & $\begin{array}{c}245.9 \\
(151 \cdot 1 \text { to } \\
378 \cdot 7)\end{array}$ & $-2.7(-3.9 \text { to }-1.5)^{*}$ \\
\hline Deciduous caries & $\begin{array}{c}173 \cdot 7 \\
(75 \cdot 1 \text { to } \\
335 \cdot 6)\end{array}$ & $\begin{array}{c}181 \cdot 1 \\
(79.0 \text { to } \\
350.9)\end{array}$ & $4.2(2.7 \text { to } 5.9)^{*}$ & $\begin{array}{l}2 \cdot 5(1 \cdot 1 \\
\text { to } 4 \cdot 9)\end{array}$ & $\begin{array}{l}2.5(1.1 \\
\text { to } 4.9)\end{array}$ & $-0 \cdot 2(-1 \cdot 6$ to $1 \cdot 4)$ \\
\hline Permanent caries & $\begin{array}{c}2190 \cdot 3 \\
(1007 \cdot 6 \text { to } \\
4232 \cdot 7)\end{array}$ & $\begin{array}{c}2411 \cdot 0 \\
(1102 \cdot 6 \text { to } \\
4664 \cdot 5)\end{array}$ & $10.1(8.6 \text { to } 11.5)^{*}$ & $\begin{array}{c}33 \cdot 2 \\
(15 \cdot 3 \text { to } \\
64 \cdot 0)\end{array}$ & $\begin{array}{c}33 \cdot 4 \\
(15 \cdot 3 \text { to } \\
64 \cdot 5)\end{array}$ & $0.5(-0.9$ to 1.8$)$ \\
\hline Periodontal diseases & $\begin{array}{c}2748 \cdot 2 \\
(1103 \cdot 3 \text { to } \\
5617 \cdot 2)\end{array}$ & $\begin{array}{c}3286 \cdot 0 \\
(1318 \cdot 3 \text { to } \\
6750 \cdot 3)\end{array}$ & $19.6(17.2 \text { to } 22.0)^{*}$ & $\begin{array}{c}47 \cdot 2 \\
(19 \cdot 0 \text { to } \\
96 \cdot 4)\end{array}$ & $\begin{array}{c}47.7 \\
(19 \cdot 1 \text { to } \\
97.9)\end{array}$ & $1.0(-1 \cdot 0$ to $3 \cdot 0)$ \\
\hline
\end{tabular}




\begin{tabular}{|c|c|c|c|c|c|c|}
\hline & \multicolumn{3}{|c|}{ All ages DALYs (thousands) ${ }^{*}$} & \multicolumn{3}{|c|}{ Age-standardised DALYs (per 100000 ) } \\
\hline & 2005 & 2013 & Percentage change & 2005 & 2013 & Percentage change \\
\hline Edentulism and severe tooth & $\begin{array}{c}5953 \cdot 6 \\
(4032 \cdot 0 \text { to } \\
8148 \cdot 1)\end{array}$ & $\begin{array}{l}6855 \cdot 6 \\
(4647 \cdot 2 \text { to } \\
9420 \cdot 4)\end{array}$ & $15 \cdot 2(13.1 \text { to } 17 \cdot 2)^{*}$ & $\begin{array}{c}117.6 \\
(79.7 \text { to } \\
160 \cdot 8)\end{array}$ & $\begin{array}{c}110 \cdot 5 \\
(75 \cdot 0 \text { to } \\
151 \cdot 7)\end{array}$ & $-6 \cdot 1(-7 \cdot 6 \text { to }-4 \cdot 5)^{*}$ \\
\hline Other oral disorders & $\begin{array}{c}3319.4 \\
(2096 \cdot 9 \text { to } \\
4915 \cdot 4)\end{array}$ & $\begin{array}{c}3715 \cdot 7 \\
(2347 \cdot 5 \text { to } \\
5558 \cdot 6)\end{array}$ & $11.9(10 \cdot 2 \text { to } 13 \cdot 8)^{*}$ & $\begin{array}{c}52 \cdot 0 \\
(32 \cdot 9 \text { to } \\
77 \cdot 0)\end{array}$ & $\begin{array}{c}51.9 \\
(32 \cdot 8 \text { to } \\
77 \cdot 6)\end{array}$ & $-0 \cdot 2(-1.7$ to $1 \cdot 4)$ \\
\hline Sudden infant death syndrome & $\begin{array}{l}1313 \cdot 3 \\
(860 \cdot 2 \text { to } \\
2147 \cdot 1)\end{array}$ & $\begin{array}{l}1299.5 \\
(828 \cdot 6 \text { to } \\
1849 \cdot 5)\end{array}$ & $0 \cdot 6(-27 \cdot 3$ to $25 \cdot 2)$ & $\begin{array}{c}18 \cdot 8 \\
(12 \cdot 3 \text { to } \\
30 \cdot 8)\end{array}$ & $\begin{array}{l}17 \cdot 6 \\
(11 \cdot 2 \text { to } \\
25 \cdot 0)\end{array}$ & $-5 \cdot 1(-31 \cdot 4$ to $18 \cdot 1)$ \\
\hline Injuries & $\begin{array}{c}267681 \cdot 2 \\
(250 \\
424 \cdot 7 \text { to } \\
283 \\
221 \cdot 0)\end{array}$ & $\begin{array}{c}247582 \cdot 4 \\
(231 \\
253 \cdot 2 \text { to } \\
265 \\
122 \cdot 7)\end{array}$ & $-7 \cdot 6(-11 \cdot 7 \text { to }-2 \cdot 8)^{*}$ & $\begin{array}{c}4166 \cdot 9 \\
(3895 \cdot 0 \\
\text { to } \\
4427 \cdot 3)\end{array}$ & $\begin{array}{c}3464 \cdot 2 \\
(3234 \cdot 7 \\
\text { to } \\
3720 \cdot 4)\end{array}$ & $\begin{array}{c}-17 \cdot 0(-20 \cdot 6 \text { to } \\
-12 \cdot 3)^{*}\end{array}$ \\
\hline Transport injuries & $\begin{array}{c}82941 \cdot 2 \\
(75570 \cdot 2 \\
\text { to } 87 \\
805 \cdot 0)\end{array}$ & $\begin{array}{c}78952 \cdot 9 \\
(72122 \cdot 8 \\
\text { to } 85 \\
115 \cdot 6)\end{array}$ & $-4.9(-9 \cdot 8$ to $0 \cdot 7)$ & $\begin{array}{c}1270 \cdot 5 \\
(1154 \cdot 4 \\
\text { to } \\
1349 \cdot 4)\end{array}$ & $\begin{array}{l}1092 \cdot 2 \\
(998 \cdot 8 \text { to } \\
1177 \cdot 4)\end{array}$ & $\begin{array}{c}-14 \cdot 1(-18 \cdot 5 \text { to } \\
-9 \cdot 1)^{*}\end{array}$ \\
\hline Road injuries & $\begin{array}{c}76626 \cdot 7 \\
(70107 \cdot 3 \\
\text { to } 81 \\
166 \cdot 5)\end{array}$ & $\begin{array}{c}73251 \cdot 1 \\
(66857 \cdot 1 \\
\text { to } 78 \\
671 \cdot 0)\end{array}$ & $-4 \cdot 4(-9 \cdot 5$ to $1 \cdot 0)$ & $\begin{array}{c}1171 \cdot 6 \\
(1069 \cdot 3 \\
\text { to } \\
1242 \cdot 2)\end{array}$ & $\begin{array}{c}1012.5 \\
(923 \cdot 7 \text { to } \\
1087 \cdot 5)\end{array}$ & $\begin{array}{c}-13 \cdot 6(-18 \cdot 1 \text { to } \\
-8 \cdot 7)^{*}\end{array}$ \\
\hline Pedestrian road injuries & $\begin{array}{c}26400 \cdot 1 \\
(22734 \cdot 6 \\
\text { to } 30 \\
607 \cdot 2)\end{array}$ & $\begin{array}{c}25580 \cdot 0 \\
(21221 \cdot 0 \\
\text { to } 29 \\
568 \cdot 6)\end{array}$ & $-2 \cdot 9(-10 \cdot 5$ to $4 \cdot 7)$ & $\begin{array}{c}408 \cdot 7 \\
(352 \cdot 0 \text { to } \\
471 \cdot 8)\end{array}$ & $\begin{array}{c}358 \cdot 0 \\
(297 \cdot 3 \text { to } \\
412 \cdot 8)\end{array}$ & $\begin{array}{c}-12 \cdot 2(-19 \cdot 0 \text { to } \\
-5 \cdot 5)^{*}\end{array}$ \\
\hline Cyclist road injuries & $\begin{array}{c}5010 \cdot 9 \\
(4305 \cdot 2 \text { to } \\
5689 \cdot 1)\end{array}$ & $\begin{array}{c}4701 \cdot 5 \\
(4005 \cdot 2 \text { to } \\
5424 \cdot 2)\end{array}$ & $-6 \cdot 4(-12 \cdot 6$ to $0 \cdot 7)$ & $\begin{array}{c}78.5 \\
(67.6 \text { to } \\
88.9)\end{array}$ & $\begin{array}{l}66 \cdot 2 \\
(56 \cdot 5 \text { to } \\
76 \cdot 3)\end{array}$ & $\begin{array}{c}-15 \cdot 9(-21 \cdot 4 \text { to } \\
-9 \cdot 4)^{*}\end{array}$ \\
\hline Motorcyclist road injuries & $\begin{array}{c}15234 \cdot 5 \\
(12694 \cdot 2 \\
\text { to } 17 \\
550 \cdot 9)\end{array}$ & $\begin{array}{c}14199.2 \\
(11743.8 \\
\text { to } 16 \\
579.2)\end{array}$ & $-7 \cdot 0(-13 \cdot 6$ to $0 \cdot 7)$ & $\begin{array}{c}227 \cdot 8 \\
(190 \cdot 0 \text { to } \\
262 \cdot 2)\end{array}$ & $\begin{array}{c}192 \cdot 8 \\
(159 \cdot 4 \text { to } \\
225 \cdot 1)\end{array}$ & $\begin{array}{c}-15 \cdot 5(-21 \cdot 5 \text { to } \\
-8 \cdot 6)^{*}\end{array}$ \\
\hline Motor vehicle road injuries & $\begin{array}{c}28677 \cdot 5 \\
(25364 \cdot 5 \\
\text { to } 31 \\
686 \cdot 1)\end{array}$ & $\begin{array}{c}27692 \cdot 3 \\
(24232 \cdot 9 \\
\text { to } 30 \\
737 \cdot 9)\end{array}$ & $-3.4(-8.0$ to 1.4$)$ & $\begin{array}{c}436 \cdot 9 \\
(386 \cdot 5 \text { to } \\
482 \cdot 5)\end{array}$ & $\begin{array}{c}380 \cdot 6 \\
(333 \cdot 3 \text { to } \\
422 \cdot 3)\end{array}$ & $\begin{array}{c}-12 \cdot 9(-16 \cdot 9 \text { to } \\
-8 \cdot 7)^{*}\end{array}$ \\
\hline Other road injuries & $\begin{array}{c}1303 \cdot 7 \\
(971 \cdot 1 \text { to } \\
1666 \cdot 4)\end{array}$ & $\begin{array}{c}1078 \cdot 1 \\
(779.9 \text { to } \\
1337 \cdot 0)\end{array}$ & $\begin{array}{c}-17 \cdot 6(-28 \cdot 2 \text { to } \\
-2 \cdot 3)^{*}\end{array}$ & $\begin{array}{l}19 \cdot 7 \\
(14 \cdot 7 \text { to } \\
25 \cdot 2)\end{array}$ & $\begin{array}{c}14 \cdot 9 \\
(10 \cdot 8 \text { to } \\
18 \cdot 5)\end{array}$ & $\begin{array}{c}-24 \cdot 5(-34 \cdot 3 \text { to } \\
-11 \cdot 3)^{*}\end{array}$ \\
\hline Other transport injuries & $\begin{array}{c}6314 \cdot 5 \\
(5315 \cdot 6 \text { to } \\
7082 \cdot 6)\end{array}$ & $\begin{array}{c}5701 \cdot 8 \\
(4908 \cdot 3 \text { to } \\
6443 \cdot 6)\end{array}$ & $\begin{array}{c}-10 \cdot 1(-15 \cdot 9 \text { to } \\
-0 \cdot 8)^{*}\end{array}$ & $\begin{array}{l}98 \cdot 8 \\
(83 \cdot 0 \text { to } \\
111 \cdot 4)\end{array}$ & $\begin{array}{l}79 \cdot 7 \\
(68 \cdot 6 \text { to } \\
90 \cdot 4)\end{array}$ & $\begin{array}{c}-19 \cdot 7(-24 \cdot 6 \text { to } \\
-11 \cdot 7)^{*}\end{array}$ \\
\hline Unintentional injuries & $\begin{array}{c}112792 \cdot 0 \\
(104 \\
542 \cdot 3 \text { to } \\
121 \\
686 \cdot 5)\end{array}$ & $\begin{array}{c}105941 \cdot 3 \\
(96996 \cdot 1 \\
\text { to } 117 \\
265 \cdot 2)\end{array}$ & $-6 \cdot 3(-11 \cdot 1$ to $0 \cdot 2)$ & $\begin{array}{c}1789 \cdot 1 \\
(1651 \cdot 5 \\
\text { to } \\
1938 \cdot 7)\end{array}$ & $\begin{array}{c}1509 \cdot 4 \\
(1379 \cdot 5 \\
\text { to } \\
1673 \cdot 8)\end{array}$ & $\begin{array}{c}-15 \cdot 8(-20 \cdot 0 \text { to } \\
-10 \cdot 1)^{*}\end{array}$ \\
\hline Falls & $\begin{array}{c}26950 \cdot 1 \\
(22901 \cdot 1 \\
\text { to } 31 \\
245 \cdot 3)\end{array}$ & $\begin{array}{c}27491.4 \\
(23388.5 \\
\text { to } 31 \\
888.5)\end{array}$ & $2 \cdot 1(-4 \cdot 0$ to $7 \cdot 6)$ & $\begin{array}{c}480 \cdot 3 \\
(407.5 \text { to } \\
561 \cdot 2)\end{array}$ & $\begin{array}{c}415 \cdot 4 \\
(352 \cdot 5 \text { to } \\
483 \cdot 2)\end{array}$ & $\begin{array}{c}-13 \cdot 5(-18.4 \text { to } \\
-9 \cdot 0)^{*}\end{array}$ \\
\hline Drowning & $\begin{array}{c}25529 \cdot 1 \\
(21942 \cdot 1 \\
\text { to } 29 \\
975 \cdot 8)\end{array}$ & $\begin{array}{c}21608 \cdot 0 \\
(18192 \cdot 8 \\
\text { to } 29 \\
799 \cdot 1)\end{array}$ & $-17 \cdot 3(-23 \cdot 9$ to $4 \cdot 0)$ & $\begin{array}{c}376 \cdot 2 \\
(323 \cdot 4 \text { to } \\
441.8)\end{array}$ & $\begin{array}{l}297 \cdot 7 \\
(250 \cdot 8 \text { to } \\
410 \cdot 4)\end{array}$ & $\begin{array}{c}-22 \cdot 6(-28 \cdot 7 \text { to } \\
-2 \cdot 8)^{*}\end{array}$ \\
\hline
\end{tabular}




\begin{tabular}{|c|c|c|c|c|c|c|}
\hline & \multicolumn{3}{|c|}{ All ages DALYs (thousands) ${ }^{*}$} & \multicolumn{3}{|c|}{ Age-standardised DALYs (per 100000$)^{*}$} \\
\hline & 2005 & 2013 & Percentage change & 2005 & 2013 & Percentage change \\
\hline Fire, heat, and hot substances & $\begin{array}{l}13280 \cdot 4 \\
(11575 \cdot 9 \\
\text { to } 15 \\
303 \cdot 6)\end{array}$ & $\begin{array}{c}12314 \cdot 8 \\
(10493 \cdot 2 \\
\text { to } 14 \\
700 \cdot 0)\end{array}$ & $-7 \cdot 8(-20 \cdot 3$ to $11 \cdot 5)$ & $\begin{array}{l}202 \cdot 5 \\
(176 \cdot 3 \text { to } \\
232 \cdot 5)\end{array}$ & $\begin{array}{c}170 \cdot 8 \\
(145 \cdot 7 \text { to } \\
203 \cdot 9)\end{array}$ & $-16 \cdot 2(-27 \cdot 1$ to $0 \cdot 7)$ \\
\hline Poisonings & $\begin{array}{c}5492 \cdot 5 \\
(4200 \cdot 6 \text { to } \\
6146 \cdot 7)\end{array}$ & $\begin{array}{c}4535 \cdot 6 \\
(3221 \cdot 0 \text { to } \\
5172 \cdot 8)\end{array}$ & $\begin{array}{c}-17 \cdot 4(-25 \cdot 8 \text { to } \\
-9 \cdot 0)^{*}\end{array}$ & $\begin{array}{c}83 \cdot 4 \\
(64 \cdot 0 \text { to } \\
93 \cdot 1)\end{array}$ & $\begin{array}{c}62 \cdot 7 \\
(44 \cdot 6 \text { to } \\
71 \cdot 5)\end{array}$ & $\begin{array}{c}-24.8(-32.6 \text { to } \\
-17 \cdot 2)^{*}\end{array}$ \\
\hline Exposure to mechanical forces & $\begin{array}{c}14798 \cdot 4 \\
(13371 \cdot 9 \\
\text { to } 16 \\
946 \cdot 5)\end{array}$ & $\begin{array}{c}14037 \cdot 9 \\
(12407 \cdot 2 \\
\text { to } 17 \\
176 \cdot 1)\end{array}$ & $-6 \cdot 1(-11 \cdot 5$ to $6 \cdot 0)$ & $\begin{array}{c}226 \cdot 7 \\
(204 \cdot 2 \text { to } \\
259 \cdot 5)\end{array}$ & $\begin{array}{c}194 \cdot 8 \\
(172 \cdot 2 \text { to } \\
237 \cdot 8)\end{array}$ & $\begin{array}{c}-14 \cdot 9(-19 \cdot 6 \text { to } \\
-3 \cdot 8)^{*}\end{array}$ \\
\hline Unintentional firearm injuries & $\begin{array}{c}2843 \cdot 7 \\
(2559 \cdot 5 \text { to } \\
3173 \cdot 4)\end{array}$ & $\begin{array}{c}2502 \cdot 6 \\
(2193 \cdot 8 \text { to } \\
2959 \cdot 6)\end{array}$ & $-12 \cdot 2(-23 \cdot 2$ to $1 \cdot 8)$ & $\begin{array}{c}42 \cdot 5 \\
(38 \cdot 3 \text { to } \\
47 \cdot 3)\end{array}$ & $\begin{array}{c}34 \cdot 2 \\
(30 \cdot 0 \text { to } \\
40 \cdot 2)\end{array}$ & $\begin{array}{c}-19.8(-29.5 \text { to } \\
-7.2)^{*}\end{array}$ \\
\hline Unintentional suffocation & $\begin{array}{c}2365 \cdot 8 \\
(1972 \cdot 6 \text { to } \\
3969 \cdot 2)\end{array}$ & $\begin{array}{c}2586 \cdot 9 \\
(1941 \cdot 6 \text { to } \\
5623 \cdot 3)\end{array}$ & $4 \cdot 2(-13 \cdot 5$ to $51 \cdot 0)$ & $\begin{array}{c}34 \cdot 5 \\
(28 \cdot 8 \text { to } \\
57 \cdot 8)\end{array}$ & $\begin{array}{c}35 \cdot 4 \\
(26 \cdot 6 \text { to } \\
76 \cdot 8)\end{array}$ & $-2 \cdot 4(-18 \cdot 9$ to $41 \cdot 8)$ \\
\hline $\begin{array}{l}\text { Other exposure to mechanical } \\
\text { forces }\end{array}$ & $\begin{array}{c}9588 \cdot 9 \\
(8377 \cdot 2 \text { to } \\
11050 \cdot 4)\end{array}$ & $\begin{array}{c}8948 \cdot 4 \\
(7781 \cdot 4 \text { to } \\
10308 \cdot 1)\end{array}$ & $-6 \cdot 7(-12 \cdot 1 \text { to }-1 \cdot 3)^{*}$ & $\begin{array}{c}149 \cdot 6 \\
(130 \cdot 2 \text { to } \\
173 \cdot 0)\end{array}$ & $\begin{array}{c}125 \cdot 3 \\
(108 \cdot 6 \text { to } \\
144 \cdot 8)\end{array}$ & $\begin{array}{c}-16 \cdot 3(-20 \cdot 9 \text { to } \\
-11 \cdot 6)^{*}\end{array}$ \\
\hline $\begin{array}{l}\text { Adverse effects of medical } \\
\text { treatment }\end{array}$ & $\begin{array}{c}5102 \cdot 7 \\
(3964 \cdot 6 \text { to } \\
5855 \cdot 4)\end{array}$ & $\begin{array}{c}5392 \cdot 2 \\
(4125 \cdot 3 \text { to } \\
6588 \cdot 9)\end{array}$ & $5 \cdot 5(-7 \cdot 8$ to $22 \cdot 5)$ & $\begin{array}{c}81 \cdot 5 \\
(63 \cdot 3 \text { to } \\
93 \cdot 0)\end{array}$ & $\begin{array}{c}76 \cdot 9 \\
(58 \cdot 9 \text { to } \\
93 \cdot 8)\end{array}$ & $-5 \cdot 8(-17 \cdot 1$ to $8 \cdot 4)$ \\
\hline Animal contact & $\begin{array}{c}4358 \cdot 7 \\
(3411 \cdot 5 \text { to } \\
6835 \cdot 1)\end{array}$ & $\begin{array}{c}4281 \cdot 1 \\
(3418 \cdot 6 \text { to } \\
6930 \cdot 8)\end{array}$ & $-2 \cdot 7(-15 \cdot 2$ to $14 \cdot 8)$ & $\begin{array}{c}66 \cdot 5 \\
(52 \cdot 4 \text { to } \\
104 \cdot 3)\end{array}$ & $\begin{array}{c}59.7 \\
(47 \cdot 7 \text { to } \\
96 \cdot 8)\end{array}$ & $-11 \cdot 0(-22 \cdot 2$ to $5 \cdot 0)$ \\
\hline Venomous & $\begin{array}{c}3081 \cdot 3 \\
(2335 \cdot 1 \text { to } \\
5172 \cdot 1)\end{array}$ & $\begin{array}{c}3002 \cdot 4 \\
(2356 \cdot 4 \text { to } \\
5144 \cdot 3)\end{array}$ & $-3 \cdot 4(-17 \cdot 3$ to $17 \cdot 0)$ & $\begin{array}{c}46 \cdot 6 \\
(35 \cdot 5 \text { to } \\
78 \cdot 5)\end{array}$ & $\begin{array}{c}41 \cdot 7 \\
(32 \cdot 7 \text { to } \\
71 \cdot 6)\end{array}$ & $-11 \cdot 2(-23 \cdot 9$ to $7 \cdot 3)$ \\
\hline Non-venomous & $\begin{array}{c}1277 \cdot 4 \\
(965 \cdot 0 \text { to } \\
1739 \cdot 0)\end{array}$ & $\begin{array}{c}1278 \cdot 7 \\
(1012 \cdot 2 \text { to } \\
1926 \cdot 0)\end{array}$ & $-1 \cdot 5(-12 \cdot 5$ to $20 \cdot 5)$ & $\begin{array}{c}19 \cdot 9 \\
(15 \cdot 1 \text { to } \\
27 \cdot 0)\end{array}$ & $\begin{array}{c}18 \cdot 0 \\
(14 \cdot 3 \text { to } \\
27 \cdot 1)\end{array}$ & $-10 \cdot 7(-20 \cdot 4$ to $8 \cdot 6)$ \\
\hline Foreign body & $\begin{array}{c}7331 \cdot 3 \\
(5388 \cdot 3 \text { to } \\
10125 \cdot 9)\end{array}$ & $\begin{array}{c}6988 \cdot 8 \\
(4964 \cdot 4 \text { to } \\
9369 \cdot 6)\end{array}$ & $-4 \cdot 9(-16 \cdot 4$ to $8 \cdot 1)$ & $\begin{array}{c}112 \cdot 8 \\
(82 \cdot 5 \text { to } \\
154 \cdot 6)\end{array}$ & $\begin{array}{l}98 \cdot 5 \\
(69 \cdot 8 \text { to } \\
131 \cdot 8)\end{array}$ & $\begin{array}{c}-12.9(-22.9 \text { to } \\
-1.4)^{*}\end{array}$ \\
\hline $\begin{array}{l}\text { Pulmonary aspiration and } \\
\text { foreign body in airway }\end{array}$ & $\begin{array}{c}6999 \cdot 5 \\
(5046 \cdot 8 \text { to } \\
9797 \cdot 1)\end{array}$ & $\begin{array}{c}6633 \cdot 1 \\
(4586 \cdot 4 \text { to } \\
8996 \cdot 4)\end{array}$ & $-5 \cdot 5(-17 \cdot 1$ to $8 \cdot 1)$ & $\begin{array}{c}107 \cdot 4 \\
(77 \cdot 4 \text { to } \\
149 \cdot 2)\end{array}$ & $\begin{array}{c}93 \cdot 4 \\
(64 \cdot 4 \text { to } \\
126 \cdot 4)\end{array}$ & $\begin{array}{c}-13 \cdot 2(-23 \cdot 5 \text { to } \\
-1 \cdot 2)^{*}\end{array}$ \\
\hline Foreign body in eyes & $\begin{array}{l}56 \cdot 4(33 \cdot 4 \\
\text { to } 85 \cdot 5)\end{array}$ & $\begin{array}{l}60 \cdot 3(35 \cdot 0 \\
\text { to } 91 \cdot 7)\end{array}$ & $6.9(3.8 \text { to } 9.6)^{*}$ & $\begin{array}{l}0.9(0 \cdot 5 \\
\text { to } 1 \cdot 4)\end{array}$ & $\begin{array}{l}0.9(0 \cdot 5 \\
\text { to } 1 \cdot 3)\end{array}$ & $-5.5(-9.0 \text { to }-2 \cdot 8)^{*}$ \\
\hline Foreign body in other body part & $\begin{array}{c}275 \cdot 3 \\
(225 \cdot 5 \text { to } \\
344 \cdot 5)\end{array}$ & $\begin{array}{c}295 \cdot 4 \\
(241 \cdot 7 \text { to } \\
384 \cdot 4)\end{array}$ & $7 \cdot 5(-1 \cdot 7$ to $15 \cdot 7)$ & $\begin{array}{l}4 \cdot 5(3 \cdot 7 \\
\text { to } 5 \cdot 5)\end{array}$ & $\begin{array}{l}4 \cdot 2(3 \cdot 5 \\
\text { to } 5 \cdot 5)\end{array}$ & $-5 \cdot 4(-13 \cdot 0$ to $2 \cdot 2)$ \\
\hline Other unintentional injuries & $\begin{array}{l}9948 \cdot 8 \\
(9059 \cdot 1 \text { to } \\
10862 \cdot 9)\end{array}$ & $\begin{array}{c}9291 \cdot 4 \\
(8357 \cdot 0 \text { to } \\
10332 \cdot 8)\end{array}$ & $-6 \cdot 8(-12 \cdot 5$ to $0 \cdot 4)$ & $\begin{array}{c}159 \cdot 1 \\
(144 \cdot 0 \text { to } \\
175 \cdot 0)\end{array}$ & $\begin{array}{c}132 \cdot 8 \\
(118 \cdot 9 \text { to } \\
148 \cdot 0)\end{array}$ & $\begin{array}{c}-16 \cdot 7(-21 \cdot 7 \text { to } \\
-10 \cdot 5)\end{array}$ \\
\hline $\begin{array}{l}\text { Self-harm and interpersonal } \\
\text { violence }\end{array}$ & $\begin{array}{c}60826 \cdot 5 \\
(51784 \cdot 1 \\
\text { to } 65 \\
431 \cdot 4)\end{array}$ & $\begin{array}{c}56574 \cdot 6 \\
(48677 \cdot 7 \\
\text { to } 63 \\
256 \cdot 5)\end{array}$ & $-7 \cdot 1(-13 \cdot 5$ to $1 \cdot 2)$ & $\begin{array}{c}925 \cdot 1 \\
(787 \cdot 0 \text { to } \\
993 \cdot 3)\end{array}$ & $\begin{array}{c}773 \cdot 4 \\
(665 \cdot 2 \text { to } \\
864 \cdot 1)\end{array}$ & $\begin{array}{c}-16 \cdot 5(-22 \cdot 3 \text { to } \\
-9 \cdot 0)^{*}\end{array}$ \\
\hline Self-harm & $\begin{array}{c}37921 \cdot 9 \\
(31030 \cdot 3 \\
\text { to } 40 \\
888 \cdot 4)\end{array}$ & $\begin{array}{c}35170 \cdot 4 \\
(29194 \cdot 0 \\
\text { to } 39 \\
484 \cdot 9)\end{array}$ & $-7 \cdot 5(-15 \cdot 9$ to $3 \cdot 5)$ & $\begin{array}{c}584 \cdot 8 \\
(478 \cdot 8 \text { to } \\
629 \cdot 8)\end{array}$ & $\begin{array}{c}484 \cdot 3 \\
(403 \cdot 8 \text { to } \\
542 \cdot 8)\end{array}$ & $\begin{array}{c}-17 \cdot 4(-24 \cdot 7 \text { to } \\
-7 \cdot 7)^{*}\end{array}$ \\
\hline Interpersonal violence & $\begin{array}{l}22904 \cdot 6 \\
(17216 \cdot 9\end{array}$ & $\begin{array}{l}21404 \cdot 2 \\
(16041 \cdot 0\end{array}$ & $-6 \cdot 8(-12 \cdot 2$ to $1 \cdot 0)$ & $\begin{array}{c}340 \cdot 3 \\
(255 \cdot 2 \text { to } \\
405 \cdot 6)\end{array}$ & $\begin{array}{c}289 \cdot 1 \\
(216 \cdot 7 \text { to } \\
347 \cdot 3)\end{array}$ & $\begin{array}{c}-15 \cdot 3(-20 \cdot 2 \text { to } \\
-8 \cdot 2)^{*}\end{array}$ \\
\hline
\end{tabular}




\begin{tabular}{|c|c|c|c|c|c|c|}
\hline & \multicolumn{3}{|c|}{ All ages DALYs (thousands) ${ }^{*}$} & \multicolumn{3}{|c|}{ Age-standardised DALYs (per 100 000) } \\
\hline & 2005 & 2013 & Percentage change & 2005 & 2013 & Percentage change \\
\hline & $\begin{array}{c}\text { to } 27 \\
308 \cdot 2)\end{array}$ & $\begin{array}{l}\text { to } 25 \\
695 \cdot 2)\end{array}$ & & & & \\
\hline Assault by firearm & $\begin{array}{c}9378 \cdot 3 \\
(6442 \cdot 6 \text { to } \\
11785 \cdot 2)\end{array}$ & $\begin{array}{c}9601 \cdot 7 \\
(6465 \cdot 3 \text { to } \\
12129 \cdot 8)\end{array}$ & $2 \cdot 0(-5 \cdot 5$ to $12 \cdot 4)$ & $\begin{array}{c}137 \cdot 6 \\
(94 \cdot 4 \text { to } \\
173 \cdot 8)\end{array}$ & $\begin{array}{c}128.9 \\
(86 \cdot 8 \text { to } \\
163 \cdot 0)\end{array}$ & $-6 \cdot 6(-13 \cdot 4$ to $2 \cdot 8)$ \\
\hline Assault by sharp object & $\begin{array}{c}6242 \cdot 7 \\
(4123 \cdot 9 \text { to } \\
8202 \cdot 9)\end{array}$ & $\begin{array}{c}5907 \cdot 0 \\
(4065 \cdot 6 \text { to } \\
8254 \cdot 7)\end{array}$ & $-6 \cdot 1(-16 \cdot 0$ to $10 \cdot 5)$ & $\begin{array}{l}92 \cdot 9 \\
(61 \cdot 4 \text { to } \\
121 \cdot 8)\end{array}$ & $\begin{array}{c}79 \cdot 7 \\
(54.9 \text { to } \\
111 \cdot 1)\end{array}$ & $-14.8(-23 \cdot 8$ to 0.2$)$ \\
\hline Assault by other means & $\begin{array}{c}7283.5 \\
(5393.8 \text { to } \\
9079.7)\end{array}$ & $\begin{array}{c}5895 \cdot 5 \\
(4268 \cdot 5 \text { to } \\
7509 \cdot 6)\end{array}$ & $\begin{array}{c}-19 \cdot 7(-25 \cdot 6 \text { to } \\
-8 \cdot 9)^{*}\end{array}$ & $\begin{array}{c}109 \cdot 9 \\
(81 \cdot 3 \text { to } \\
136 \cdot 6)\end{array}$ & $\begin{array}{c}80 \cdot 5 \\
(58 \cdot 3 \text { to } \\
102 \cdot 3)\end{array}$ & $\begin{array}{c}-27 \cdot 3(-32 \cdot 6 \text { to } \\
-17 \cdot 7)^{*}\end{array}$ \\
\hline $\begin{array}{l}\text { Forces of nature, war, and legal } \\
\text { intervention }\end{array}$ & $\begin{array}{c}11121 \cdot 5 \\
(6687 \cdot 7 \\
\text { to } 18 \\
862 \cdot 5)\end{array}$ & $\begin{array}{c}6113 \cdot 6 \\
(3504 \cdot 8 \\
\text { to } 11 \\
068 \cdot 7)\end{array}$ & $\begin{array}{c}-45 \cdot 0(-55 \cdot 3 \text { to } \\
-35 \cdot 0)^{*}\end{array}$ & $\begin{array}{c}182 \cdot 2 \\
(108 \cdot 3 \text { to } \\
312 \cdot 3)\end{array}$ & $\begin{array}{c}89 \cdot 1 \\
(50 \cdot 7 \text { to } \\
160 \cdot 0)\end{array}$ & $\begin{array}{c}-51 \cdot 2(-59 \cdot 7 \text { to } \\
-42 \cdot 9)^{*}\end{array}$ \\
\hline Exposure to forces of nature & $\begin{array}{c}4123 \cdot 6 \\
(2740 \cdot 1 \text { to } \\
7479 \cdot 8)\end{array}$ & $\begin{array}{l}1325 \cdot 5 \\
(818 \cdot 9 \text { to } \\
2516 \cdot 9)\end{array}$ & $\begin{array}{c}-69 \cdot 6(-75 \cdot 1 \text { to } \\
-51 \cdot 1)^{*}\end{array}$ & $\begin{array}{c}64 \cdot 3 \\
(42 \cdot 4 \text { to } \\
116 \cdot 3)\end{array}$ & $\begin{array}{c}19 \cdot 1 \\
(11 \cdot 8 \text { to } \\
36 \cdot 1)\end{array}$ & $\begin{array}{c}-71.9(-76.9 \text { to } \\
-55 \cdot 7)^{*}\end{array}$ \\
\hline $\begin{array}{l}\text { Collective violence and legal } \\
\text { intervention }\end{array}$ & $\begin{array}{c}6997.9 \\
(3408.9 \text { to } \\
12878.9)\end{array}$ & $\begin{array}{c}4788 \cdot 1 \\
(2602 \cdot 8 \text { to } \\
8707 \cdot 0)\end{array}$ & $\begin{array}{c}-31 \cdot 2(-47 \cdot 5 \text { to } \\
-5 \cdot 7)^{*}\end{array}$ & $\begin{array}{l}118 \cdot 0 \\
(57 \cdot 1 \text { to } \\
219 \cdot 7)\end{array}$ & $\begin{array}{c}70 \cdot 1 \\
(37 \cdot 7 \text { to } \\
128 \cdot 1)\end{array}$ & $\begin{array}{c}-40.4(-53.2 \text { to } \\
-19 \cdot 7)^{*}\end{array}$ \\
\hline
\end{tabular}

Data are DALYs (95\% UI) or \% change (95\% UI). UI=uncertainty interval. DALY=disability-adjusted life-years.

*

Percentage change is statistically significant $(\mathrm{p}<0 \cdot 05)$. 
Table 2

Decomposition of variance in 2013 global DALY rates per 100000 people for GBD level 2 causes using hierarchical regression

\begin{tabular}{|c|c|c|c|c|}
\hline & Sociodemographic status & Year & Country & Unexplained \\
\hline A.1. HIV/AIDS and tuberculosis & $20 \cdot 65 \%$ & $1 \cdot 13 \%$ & $73.09 \%$ & $5 \cdot 13 \%$ \\
\hline A.2. Diarrhoea, lower respiratory, and other common infectious diseases & $79 \cdot 14 \%$ & $0 \cdot 76 \%$ & $18 \cdot 19 \%$ & $1.91 \%$ \\
\hline A.3. Neglected tropical diseases and malaria & $14.04 \%$ & $0.08 \%$ & $84.98 \%$ & $0.91 \%$ \\
\hline A.4. Maternal disorders & $80 \cdot 34 \%$ & $0 \cdot 17 \%$ & $17 \cdot 75 \%$ & $1.74 \%$ \\
\hline A.5. Neonatal disorders & $86.90 \%$ & $0 \cdot 25 \%$ & $11.29 \%$ & $1.56 \%$ \\
\hline A.6. Nutritional deficiencies & $80 \cdot 48 \%$ & $0.06 \%$ & $17 \cdot 12 \%$ & $2 \cdot 33 \%$ \\
\hline A.7. Other communicable, maternal, neonatal, and nutritional diseases & $56 \cdot 61 \%$ & $0.52 \%$ & $40.94 \%$ & $1.94 \%$ \\
\hline B.1. Neoplasms & $15.62 \%$ & $0 \cdot 28 \%$ & $80 \cdot 91 \%$ & $3 \cdot 19 \%$ \\
\hline B.2. Cardiovascular diseases & $3.01 \%$ & $1 \cdot 19 \%$ & $88.69 \%$ & $7 \cdot 11 \%$ \\
\hline B.3. Chronic respiratory diseases & $6 \cdot 41 \%$ & $3.05 \%$ & $82.74 \%$ & $7 \cdot 80 \%$ \\
\hline B.4. Cirrhosis & $1 \cdot 18 \%$ & $0 \cdot 10 \%$ & $90.78 \%$ & $7.94 \%$ \\
\hline B.5. Digestive diseases & $17.49 \%$ & $0.96 \%$ & $76.95 \%$ & $4.60 \%$ \\
\hline B.6. Neurological disorders & $45 \cdot 38 \%$ & $0 \cdot 01 \%$ & $53.01 \%$ & $1 \cdot 61 \%$ \\
\hline B.7. Mental and substance use disorders & $28.62 \%$ & $0 \cdot 37 \%$ & $68.41 \%$ & $2 \cdot 60 \%$ \\
\hline B.8. Diabetes, urogenital, blood, and endocrine diseases & $8.77 \%$ & $1 \cdot 85 \%$ & $83.05 \%$ & $6 \cdot 33 \%$ \\
\hline B.9. Musculoskeletal disorders & $65.71 \%$ & $0.02 \%$ & $33 \cdot 30 \%$ & $0.96 \%$ \\
\hline B.10. Other non-communicable diseases & $57 \cdot 14 \%$ & $1.05 \%$ & $33.72 \%$ & $8.08 \%$ \\
\hline C.1. Transport injuries & $21 \cdot 39 \%$ & $1.76 \%$ & $63.26 \%$ & $13.58 \%$ \\
\hline C.2. Unintentional injuries & $4.58 \%$ & $6 \cdot 27 \%$ & $80 \cdot 81 \%$ & $8 \cdot 33 \%$ \\
\hline C.3. Self-harm and interpersonal violence & $2.61 \%$ & $0 \cdot 36 \%$ & $91.23 \%$ & $5 \cdot 80 \%$ \\
\hline C.4. Forces of nature, war, and legal intervention & $24.54 \%$ & $1 \cdot 18 \%$ & $55.63 \%$ & $18.65 \%$ \\
\hline
\end{tabular}

DALY=disability-adjusted life-years. $G B D=$ Global Burden of Disease. 


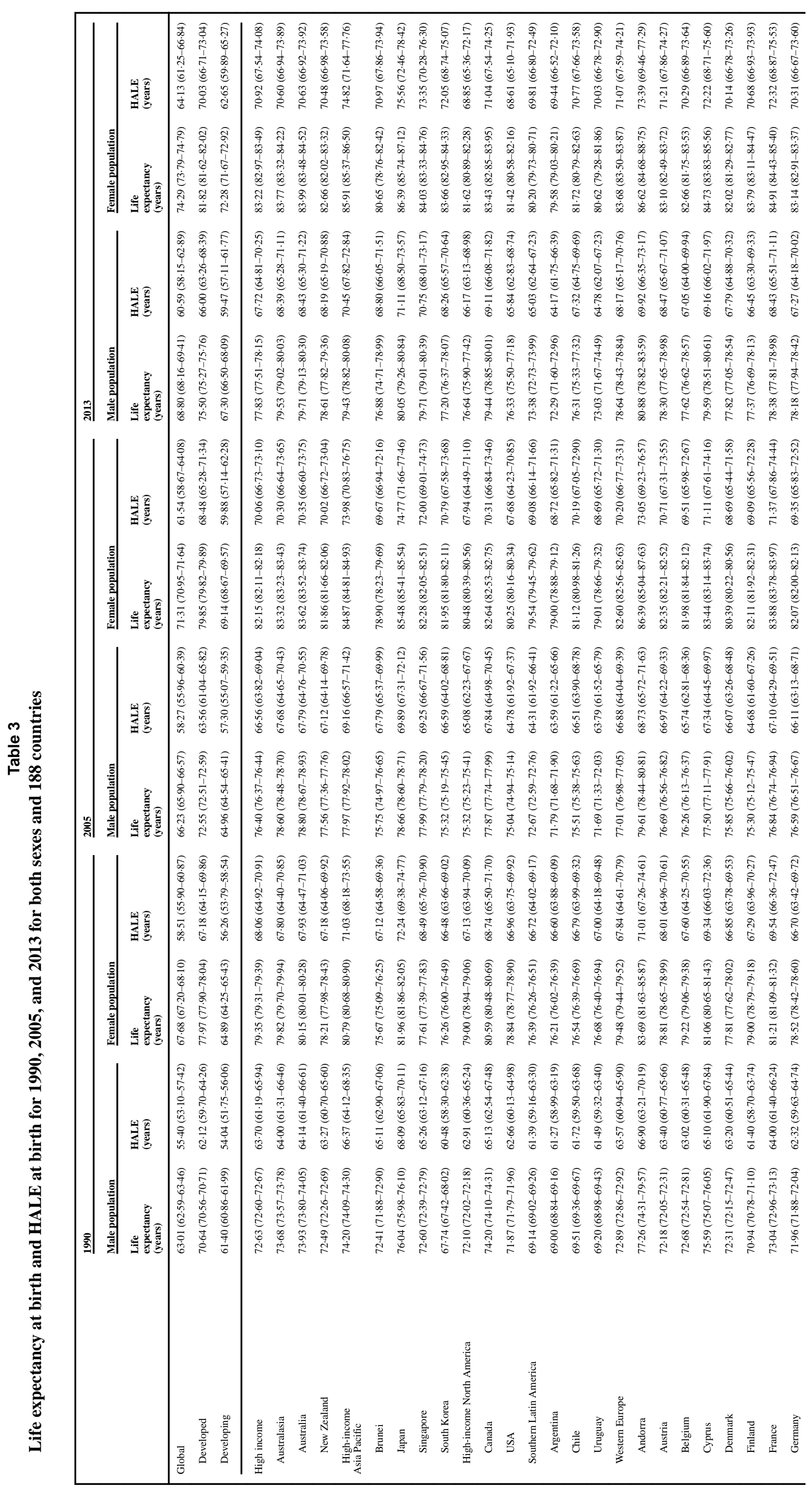




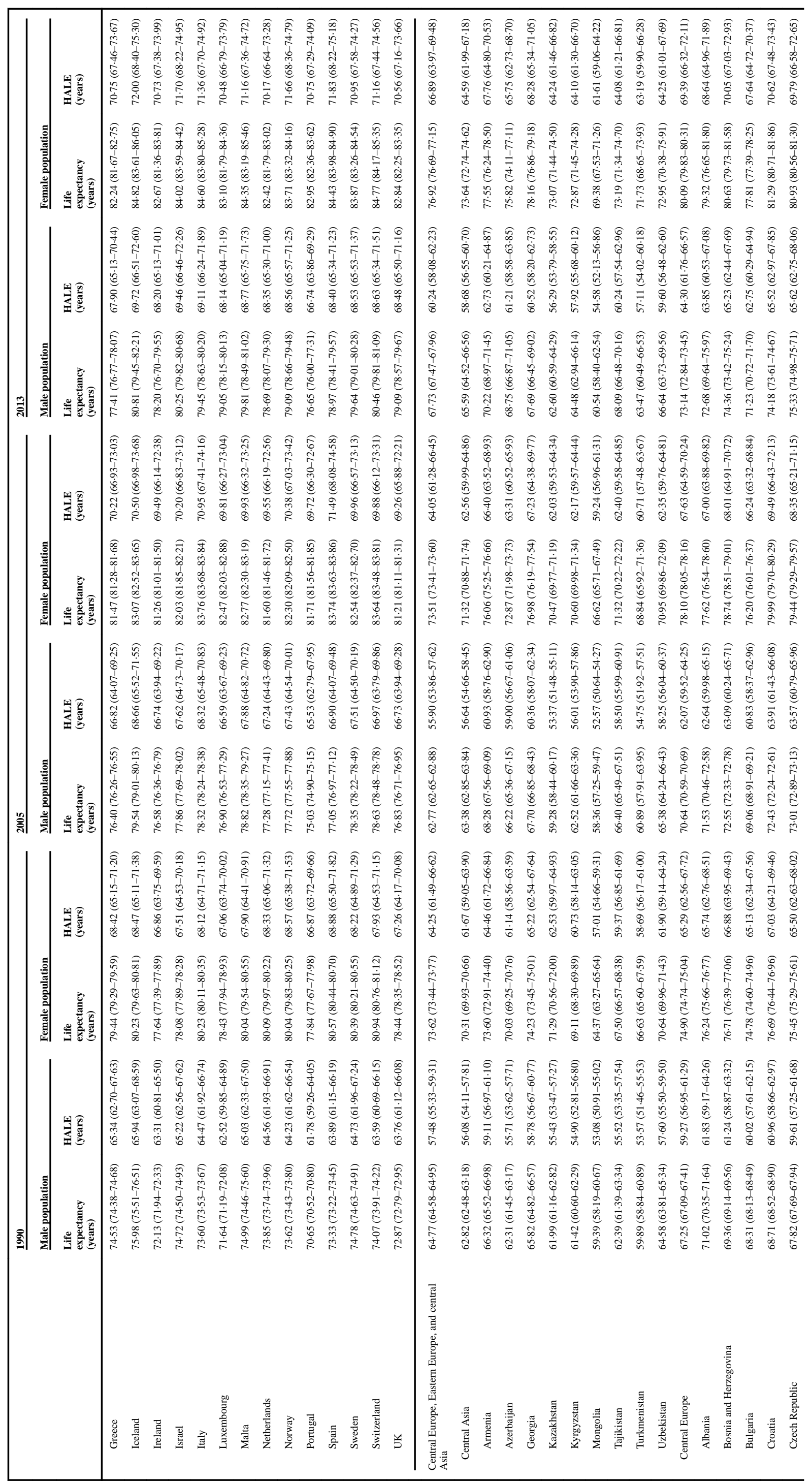




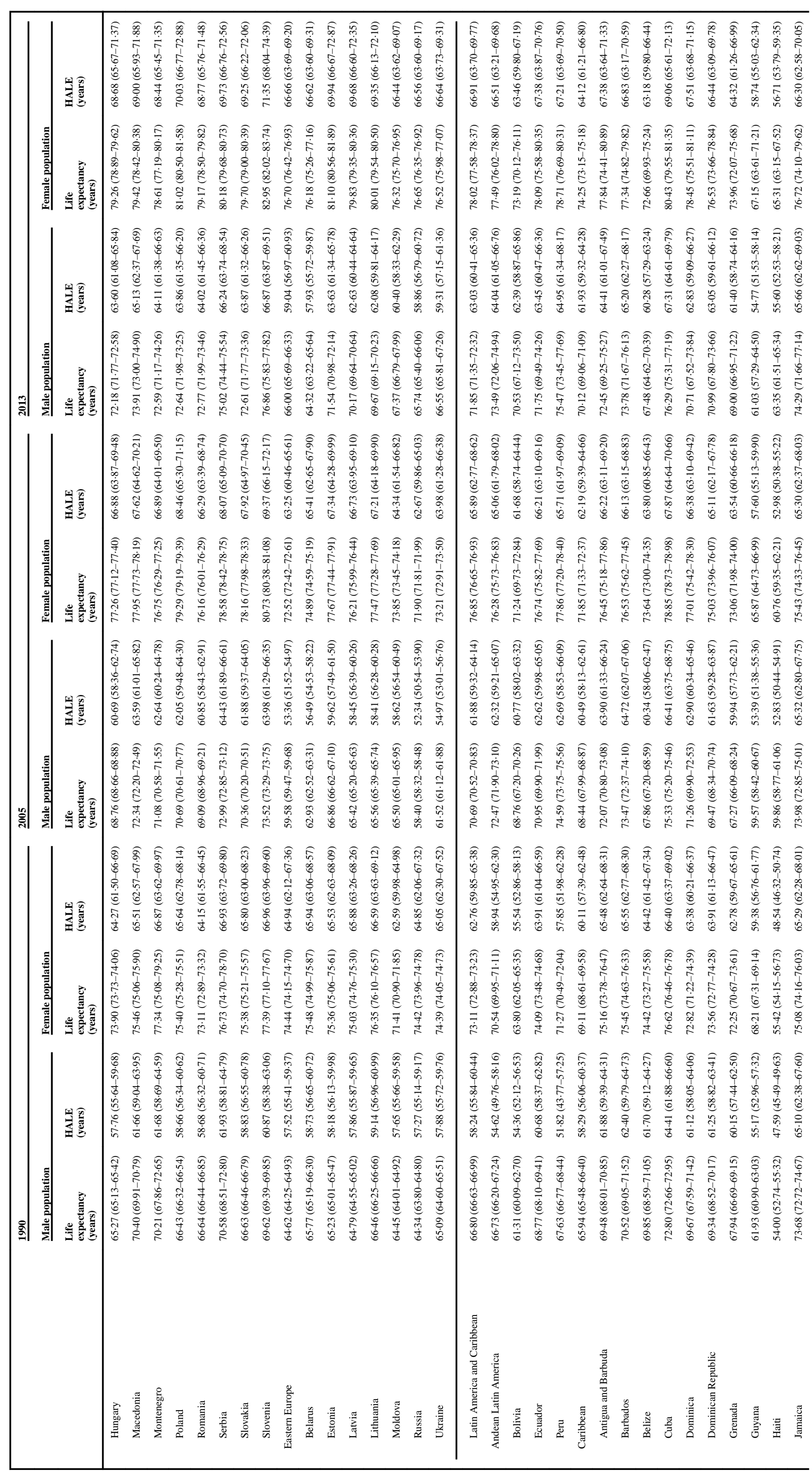




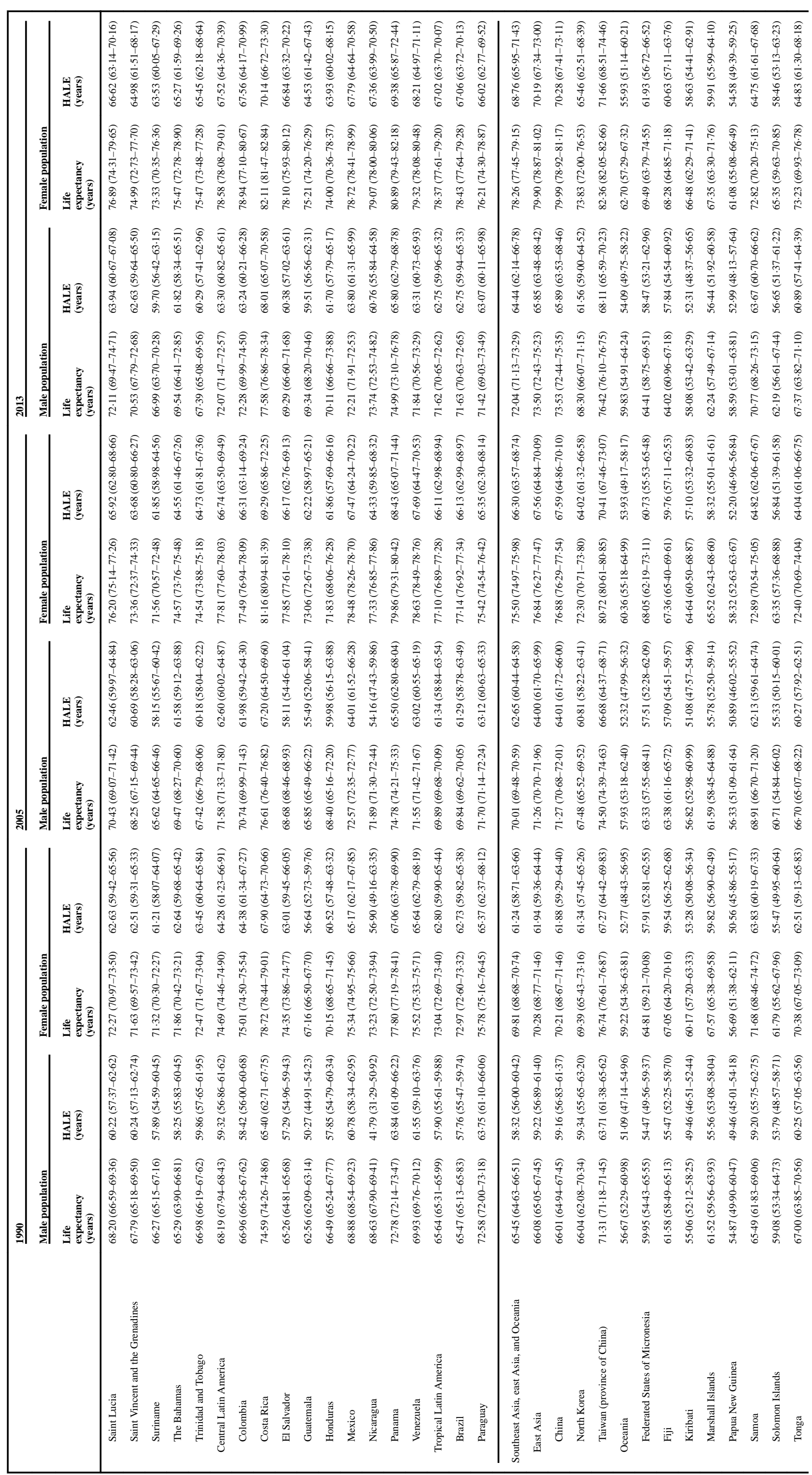




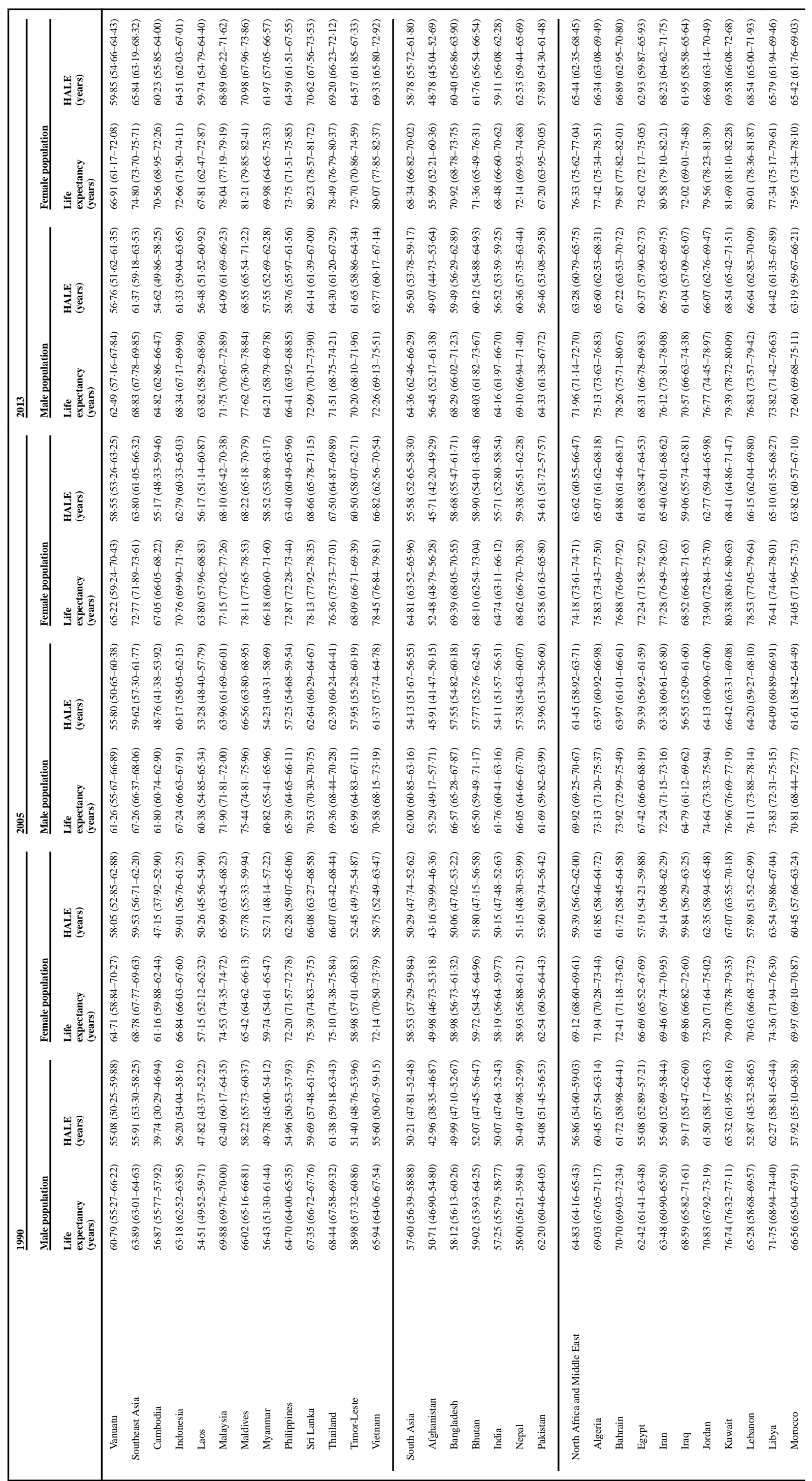




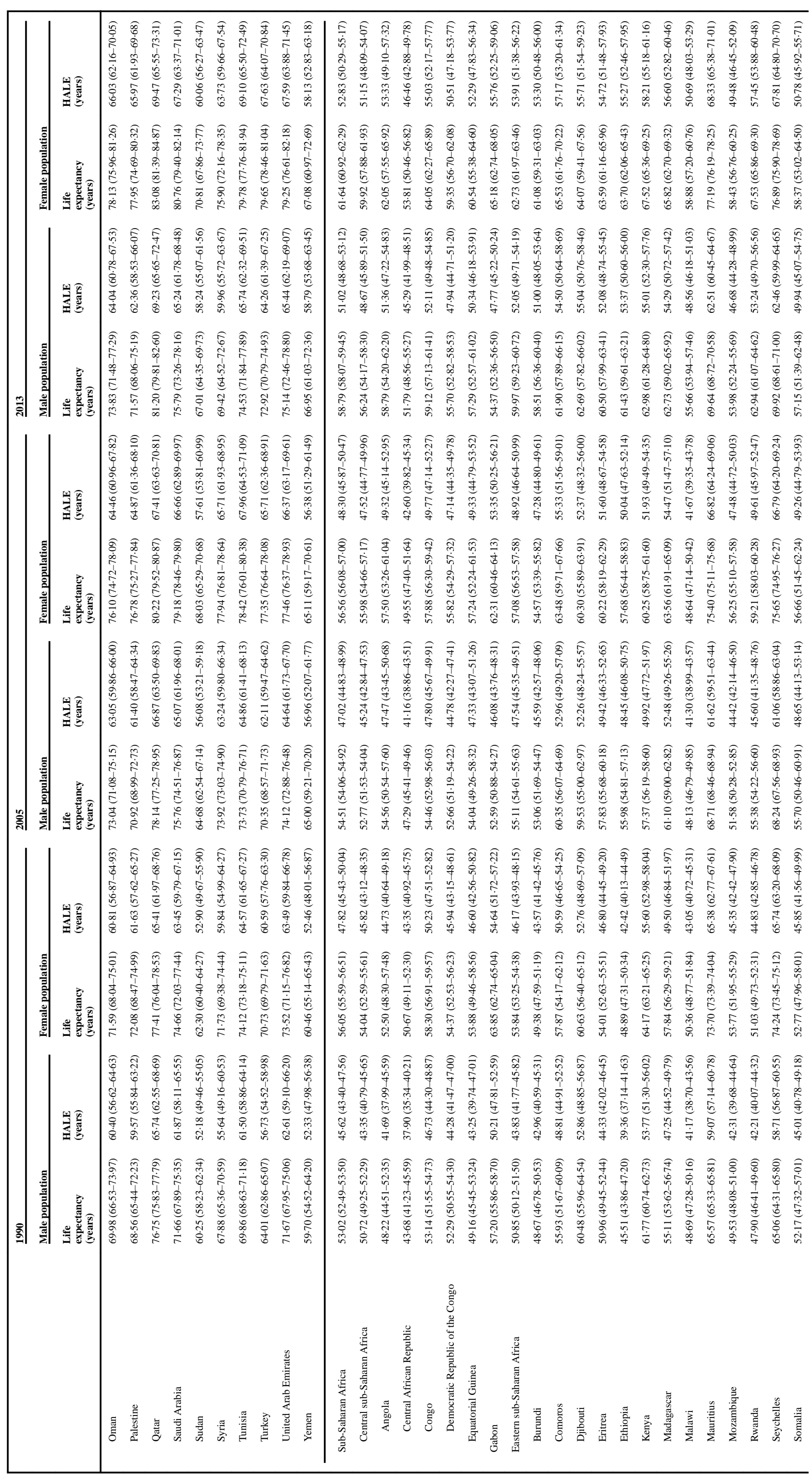




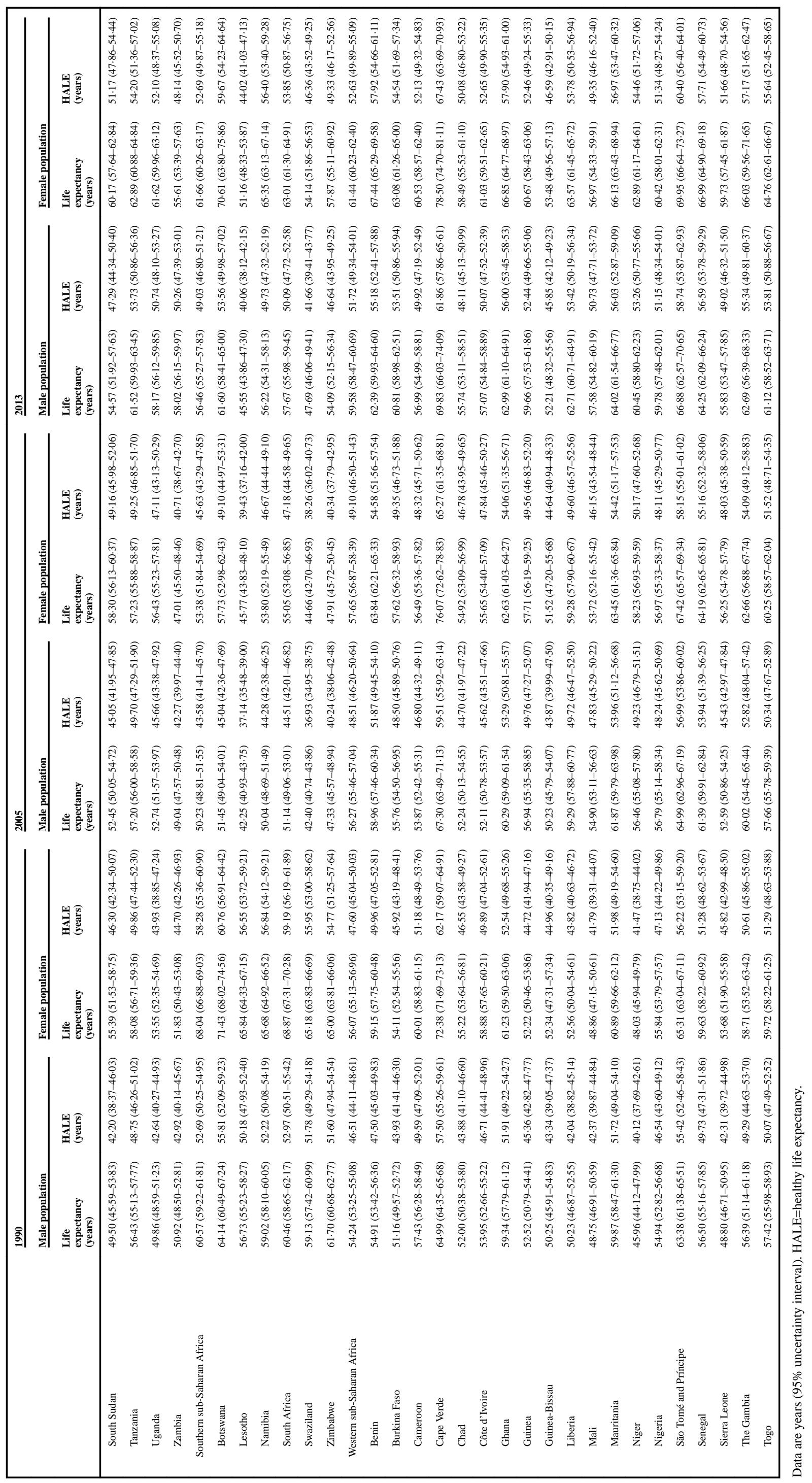

